\title{
Model identification, migration, and investment-specific shocks: \\ Three essays
}

By

Christopher A. Smith

\author{
A thesis \\ submitted to Victoria University of Wellington \\ in fulfilment of the requirements for the degree of \\ Doctor of Philosophy
}

Victoria University of Wellington

2018 


\section{Abstract}

This thesis consists of an introduction and three substantive chapters. Chapter 2 explores the identification of a small open economy model. Chapter 3 focuses on the business cycle consequences of migration. And chapter 4 investigates the contribution of investment-specific technology shocks to business cycle fluctuations in the presence of financial frictions.

Chapter 2 takes a conventional new open economy macro model for a small open economy and addresses three questions: what data series should be used to identify the parameters of such a model? Are foreign data important for the identification of domestic parameters? And lastly, which structural parameters are interdependent?

The chapter illustrates an applied methodology that enables an investigator to understand which data series are informative about parameters. The methodology can also be used to learn about the properties of the model. In particular, the methodology highlights which parameters are connected to which data series. Identification of business cycle models matters because our ability to recover structural parameters is influenced by the data series that are used to inform the estimation. Structural parameters determine both the specification of household preferences and the constraints that affect business cycle volatility, which together determine welfare. Consequently, identification analysis can provide insights into household welfare, which in turn has ramifications for the specification of monetary policy rules.

If parameters are identified then the likelihood will eventually outweigh any prior beliefs as the sample size becomes large (Gelman et al., 2004, p. 107). The approach discussed here thus shows whether data will eventually dominate prior beliefs about parameters, determining whether analysis can 
- in the limit - resolve conflicting prior beliefs, and therefore usefully inform the design of policy rules.

Chapter 3 of this thesis examines the business cycle effects that arise from an expansion of the population due to migration. In recent years, migration flows have become a highly politicised topic, both in New Zealand and abroad. While the debate on migration has become heated, comparatively little is known about the business cycle consequences of migration flows.

This chapter contributes to the macroeconomic literature by illustrating the contribution that migration shocks make to cyclical fluctuations in New Zealand, and illustrates their dynamic impact. Using an estimated dynamic stochastic general equilibrium (DSGE) model of a small open economy and a structural vector autoregression, the chapter shows that migration shocks account for a considerable portion of the variability of per capita gross domestic product (GDP). While migration shocks matter for the capital investment and consumption components of per capita GDP, other shocks are more important drivers of cyclical fluctuations in these aggregates. Migration shocks also make some contribution to residential investment and real house prices, but other shocks play a more substantial role in driving housing market volatility.

In the DSGE model, the level of human capital possessed by migrants relative to that of locals materially affects the business cycle impact of migration. The impact of migration shocks is larger when migrants have substantially different - larger or smaller - levels of human capital relative to locals. When the average migrant has higher levels of human capital than locals, as seems to be common for migrants into most $\mathrm{OECD}^{1}$ economies, a migration shock has an expansionary effect on per capita GDP and its components, which also accords with the evidence from a structural vector autoregression.

Chapter 4 of this thesis investigates the contribution of investment-specific technology (IST) shocks in driving cyclical fluctuations in a closed economy model when a borrowing constraint is introduced à la Kiyotaki and Moore (1997). IST shocks have been identified as a major driver of the business

\footnotetext{
${ }^{1}$ Organisation for Economic Cooperation and Development.
} 
cycle, eg see Greenwood et al. (2000), and Justiniano et al. (2010, 2011). These shocks affect the rate at which investment goods are transformed into capital stock, and have been linked to frictions in financial markets, because financial intermediation is instrumental in facilitating investment. The third chapter shows that the importance of these investment shocks is in fact substantially diminished when collateral constraints on firms are introduced into an estimated dynamic stochastic general equilibrium model. In the presence of binding collateral constraints, risk premium shocks, which perturb interest rates and affect intertemporal substitution, supplant IST shocks as important drivers of the business cycle. 


\section{Acknowledgements}

Chapter three of this PhD is based on coauthored work with Prof. Dr Christoph Thoenißen, and chapter four of this $\mathrm{PhD}$ is based on coauthored work with Dr Thoenißen and Dr Güneş Kamber. I would like to thank them both for allowing these papers to feature in this dissertation. Christoph and Güneş have both been very agreeable colleagues and coauthors. Thanks also to Prof. Yothin Jinjarak for taking over the supervision of this dissertation once Christoph de-camped to the University of Sheffield.

Having dispensed with the formalities, I wish to thank Victoria, Alexandra, Benjamin, and Catriona for tolerating the familial neglect that a $\mathrm{PhD}$ entails. Hopefully the scholastic achievement of the younger three will not be irreparably harmed by all the television they have watched in my absence. Victoria, of course, looked after $\frac{9}{10}$ ths of everything else in our lives. Thank you for all of your contributions Victoria - the care and attention to family, the connections to all of Wadestown (not to mention people in the Hawkes Bay and the wider Wellington region), and the enthusiasm and excitement that pervades your entire approach to life. Your resilience in the face of a considerable number of trials and tribulations is a testament to your strength of character.

I also thank my parents, Allan and Lynne Smith, for their support over many decades, and the general goodwill and assistance of my two sisters, Marian and Joan. Let me also thank Dr Paul Grimwood for providing some helpful comments on chapter 2. I also want to note a long-standing group of friends - too many to note individually - who insisted they be mentioned here. (Their contribution to this dissertation was the 'motivational' banter about the length of time it was taking...)

More seriously, let me thank the Reserve Bank of New Zealand and Dr 
C. John McDermott for providing me with the opportunity to pursue this doctorate while working at the Bank. I hope that the social benefit is strictly greater than the private benefit to me alone. Thanks also to my colleagues in the Research team, the Economics Department, and the wider Bank for providing a stimulating environment in which to work all these years. Roger Perry, a manager in various departments in the Reserve Bank, deserves especial mention. Roger passed away in April 2018 and will be greatly missed.

Lastly, the roots of this thesis extend back in time to the Johns Hopkins University in Baltimore, to Lincoln University in Canterbury, and to my undergraduate degree at Victoria University of Wellington. I greatly appreciate all the efforts that have been made to educate me over the last several decades, by friends and teachers alike. Thanks to all. 


\section{Contents}

Abstract ......................... iii

Acknowledgements ....................... vi

Table of contents . . . . . . . . . . . . . . vii

List of figures . . . . . . . . . . . . . . . . $\mathrm{x}$

List of tables . . . . . . . . . . . . . . . xiii

1 Introduction $\quad 1$

1.1 A potted history of macro modelling . . . . . . . . . . . 1

1.1.1 Empirical modelling during the 1960s and 1970s . . . . 6

1.1.2 Real business cycle and New Keynesian DSGE models 7

1.1.3 An identification detour . . . . . . . . . . . . 11

1.1.4 Model properties . . . . . . . . . . . . . 13

1.1.5 Real business cycle and New Keynesian DSGE models 15

1.1.6 The empirical performance of macro models . . . . . . 16

1.2 Criticism of the DSGE framework . . . . . . . . . . . 18

1.3 Introducing three chapters . . . . . . . . . . . . . . . 22

1.3.1 Foreign data are not necessary for identification . . . . 22

1.3.2 Skilled migration and business cycle dynamics . . . . . 24

1.3.3 Financial frictions and investment-specific technology shocks . . . . . . . . . . . . . . 26

1.4 Concluding remarks . . . . . . . . . . . . . . . 28

2 Identification of an SOE model $\quad 31$

2.1 Introduction . . . . . . . . . . . . . . . . . 31

2.2 Identification of a Bayesian DSGE model . . . . . . . . . . . . 38

2.3 Model . . . . . . . . . . . . . . . . . . . . . 50

2.4 Results . . . . . . . . . . . . . . . . . . 60 
2.4.1 Identification of foreign parameters . . . . . . . 60

2.4.2 Identification of domestic parameters . . . . . . . . 70

2.4.3 Identification of foreign parameters from domestic data 78

2.4.4 What impact do autocorrelated errors have on identification? . . . . . . . . . . . . . . 82

2.5 Conclusion . . . . . . . . . . . . . . . . 85

$\begin{array}{lr}\text { Appendices } & 89\end{array}$

2.A Iskrev theorem $2 \ldots \ldots \ldots$

2.B Inverse function theorem . . . . . . . . . . . . . . . . 89

2.C Identification of real-valued functions . . . . . . . . . . . 90

2.C.1 Identifying function . . . . . . . . . . . . 90

2.C.2 Score of a Gaussian vector autoregression . . . . . . 90

2.D DSGE solutions and identification . . . . . . . . . . . . . 92

2.E Composition of one-to-one functions is one-to-one . . . . . . 95

3 Skilled migration \& business cycles $\quad 97$

3.1 Introduction . . . . . . . . . . . . . . . . . . . 97

3.2 A model of migration in a small open economy . . . . . . . . 101

3.2.1 Households . . . . . . . . . . . . . . . . . . . 103

3.2.2 Household's first order conditions . . . . . . . . . 105

3.2 .3 Firms . . . . . . . . . . . . . . . . . 105

3.2.4 Current account . . . . . . . . . . . . . . 108

3.2.5 Driving processes . . . . . . . . . . . . . 109

3.2.6 Model description in brief . . . . . . . . . . . 110

3.3 Migration versus population growth . . . . . . . . . . . . . 110

3.4 Estimation strategy . . . . . . . . . . . . . . . . . . . . 111

3.4.1 Data . . . . . . . . . . . . . . . 112

3.4.2 Calibration and priors . . . . . . . . . . 118

3.4.3 Estimation results . . . . . . . . . . . . 118

3.5 A migration shock . . . . . . . . . . . . . . . . . . 121

3.5.1 Does migration drive the business cycle? . . . . . . . . 125

3.5.2 Non-migration properties of the model . . . . . . . . 127

3.6 Sensitivity analysis . . . . . . . . . . . . . . . . . . . . . . . . . 129

3.7 An SVAR look at the data . . . . . . . . . . . . . . . . . 132 
3.8 Conclusion . . . . . . . . . . . . . . . . . . . 136

$\begin{array}{ll}\text { Appendices } & 137\end{array}$

3.A Steady state . . . . . . . . . . . . . . . . 137

3.B MCMC convergence . . . . . . . . . . . . . . . 138

3.C Impulse response functions . . . . . . . . . . . . . . 145

3.D Historical shock decompositions . . . . . . . . . . . . 146

3.E Smoothed shocks . . . . . . . . . . . . . . . . . . . 149

4 Financial frictions \& IST shocks 151

4.1 Introduction . . . . . . . . . . . . . . . . . . . 151

4.2 Model . . . . . . . . . . . . . . . . . . . . . 153

4.2 .1 Households . . . . . . . . . . . . . . . . 155

4.2 .2 Entrepreneurs . . . . . . . . . . . . . . . . 156

4.2 .3 The rest of the model . . . . . . . . . . . . . . . 159

4.2 .4 Shocks . . . . . . . . . . . . . . . . . . 159

4.2 .5 An alternative model . . . . . . . . . . . . . . 160

4.3 Bayesian estimation . . . . . . . . . . . . . . . 160

4.4 IST and risk premium shocks and the business cycle . . . . . 166

4.4.1 IST shocks and collateral constraints . . . . . . . . . 170

4.4.2 Risk premium shocks and borrowing constraints . . . . 173

4.5 Discussion . . . . . . . . . . . . . . . . . . . . 176

4.6 Robustness over the sample . . . . . . . . . . . . . . 178

4.7 Conclusion . . . . . . . . . . . . . . . . . . . . . . . 181

$\begin{array}{ll}\text { Appendices } & 183\end{array}$

4.A Linearized model . . . . . . . . . . . . . . . . . . . . . 184

4.B.2 Data transformations . . . . . . . . . . . . . . 185

$4 . \mathrm{B}$ Data . . . . . . . . . . . . . . . . . . . 186

4.B.1 Data sources . . . . . . . . . . . . . . . . 186

4.C MCMC convergence . . . . . . . . . . . . . . . 187 


\section{List of Figures}

1.1 Identifying supply and demand curves . . . . . . . . . . . . 12

1.2 Figurative illustration of the identification problem . . . . . . 14

2.1 Two dimensional Sobol sequences and pseudo random uniform variates . . . . . . . . . . . . . . . . . . 49

2.2 Sub-maximal ranks by sample . . . . . . . . . . . . . . 76

2.3 Sub-maximal ranks vs the number of foreign variables included in the observables: Domestic parameters . . . . . . . 77

2.4 Histogram of ranks given 12 observables . . . . . . . . . . . 80

2.5 Sub-maximal ranks vs the number of foreign variables included in the observables: Foreign parameters . . . . . . . . . 81

2.6 Histogram of ranks for foreign parameter identification with/without foreign hours worked . . . . . . . . . . . . . . . 83

2.7 Histogram of ranks with/without labour market variables IID shocks . . . . . . . . . . . . . . . . . . . . . 84

3.1 Net working-age migration flows into and out of New Zealand 113

3.2 Net working-age migration flows detrended via local linear projections . . . . . . . . . . . . . . . . . . . . . 115

3.3 A migration shock (Panel A) . . . . . . . . . . . . . 122

3.4 A migration shock (Panel B) . . . . . . . . . . . . . . . . . 123

3.5 The role of migration shocks as a function of $\chi \ldots \ldots$. . . . . 131

3.6 A migration shock in a VAR . . . . . . . . . . . . . . . . . . 135

3.B.1 MCMC convergence - deciles (Panel A) . . . . . . . . . . . . 139

3.B.2 MCMC convergence - deciles (Panel B) . . . . . . . . . . . . . 140

3.B.3 MCMC convergence - deciles (Panel C) . . . . . . . . . . . . . 141

3.B.4 MCMC convergence - deciles (Panel D) . . . . . . . . . . . . 142 
3.B.5 MCMC convergence - deciles (Panel E) . . . . . . . . . . . 143

3.B.6 MCMC convergence - deciles (Panel F) . . . . . . . . . . . . 144

3.C.1Impulse responses . . . . . . . . . . . . . . . . . . . 145

3.D.1Shock decomposition - Log GDP per capita . . . . . . . . . 146

3.D.2Shock decomposition - Log consumption per capita . . . . . . 146

3.D.3Shock decomposition - Log investment per capita . . . . . . . 147

3.D.4Shock decomposition - Log residential investment per capita . 147

3.D.5Shock decomposition - Real house price . . . . . . . . . . . . 148

3.E.1Smoothed shocks . . . . . . . . . . . . . . . . . . . 149

4.1 Role of IST shock in the Great Recession in the model without borrowing constraints . . . . . . . . . . . . . . 168

4.2 The risk-premium shock and NBER recession intervals in the model with borrowing constraints . . . . . . . . . . . . . 171

4.3 Role of Risk Premium shock in the Great Recession in the model with borrowing constraints . . . . . . . . . . . . . . . 172

4.4 Impulse response to an IST shock in the model with borrowing constraints . . . . . . . . . . . . . . . . . . . 174

4.5 Impulse response to a risk premium shock in the model with borrowing constraints . . . . . . . . . . . . . 175

4.C.1MCMC convergence - deciles (Panel A) . . . . . . . . . . 188

4.C.2MCMC convergence - deciles (Panel B) . . . . . . . . . . . . . 189

4.C.3MCMC convergence - deciles (Panel C) . . . . . . . . . . . . . 190 


\section{List of Tables}

2.1 Domestic linearised equations . . . . . . . . . . . . 53

2.2 Foreign equations . . . . . . . . . . . . . . . . 54

2.3 Variable definitions . . . . . . . . . . . . . . . 55

2.4 Domestic parameter definitions . . . . . . . . . . . 56

2.5 Foreign parameter definitions . . . . . . . . . . 57

2.6 Prior distributions domestic parameters . . . . . . . . . 58

2.7 Prior distributions exogenous parameters . . . . . . . . . . . 59

2.8 Identifying $\Gamma$ parameters for foreign equations: Minimum and maximum ranks for different observables . . . . . . . . . 67

2.9 Identifying $\theta$ parameters for foreign equations: Minimum and maximum ranks for different observables . . . . . . . . . . . 71

2.10 Identifying $\Gamma$ parameters for foreign equations: Minimum and maximum ranks for different observables . . . . . . . . . . 74

2.11 Sub-maximal ranks across different combinations of observables 79

3.1 Variables - summary . . . . . . . . . . . . . . . 110

3.2 Raw data . . . . . . . . . . . . . . . . . . . 116

3.3 Data transformations . . . . . . . . . . . . . . . . . 117

3.4 Observables and model moments . . . . . . . . . . . . 117

3.5 Estimated parameters values: $\chi=1.85 \ldots \ldots$. . . . 120

3.6 Variance decomposition at the posterior mean: $\chi=1.85$. . 126

3.7 Estimated parameter values: $\chi=1 \ldots 130$

3.8 Variance decomposition at the posterior mean: $\chi=1 \ldots 132$

4.1 Estimation results for parameters and shock processes of model with borrowing constraints: 1954Q3 - 2011Q4 . . . . . . . . 162 
4.2 Estimation results for parameters and shock processes of model without borrowing constraints: 1954Q3 - 2011Q4 . . . . . . . 164

4.3 Log marginal data densities . . . . . . . . . . . . . . . . 166

4.4 Variance decomposition of model without borrowing constraints: 1954Q3 - 2011Q4 . . . . . . . . . . . . . 167

4.5 Variance decomposition of model with borrowing constraints: 1954Q3 - 2011Q4 . . . . . . . . . . . . . . 169

4.6 Estimation results for parameters and shock processes of model with borrowing constraints: 1984Q1 - 2009Q1 . . . . . . . 178

4.7 Variance decomposition of model with borrowing constraints: 1984Q1 - 2009Q1 . . . . . . . . . . . . . 181

4.A.1Linearized model equations . . . . . . . . . . . . . . . . . . 184

4.B.1Raw data . . . . . . . . . . . . . . . . . . . . 186

4.B.2 Data transformations . . . . . . . . . . . . . . . 186 


\section{Chapter 1}

\section{Introduction}

This introduction provides an elementary discussion of the history of macromodelling and describe the principles and methodology underlying dynamic stochastic general equilibrium (DSGE) models. It then summarises the three chapters that form the substantive contribution of this dissertation. The three chapters are unified by the application of dynamic stochastic general equilibrium models to business cycle fluctuations. The first chapter is primarily concerned with a methodological question regarding the choice of data used to inform estimation, while the latter two chapters focus on the drivers of business cycles.

\section{$1.1 \quad$ A potted history of macro modelling}

Macro modelling took a major step forward with the publication of Keynes' General Theory in 1936. ${ }^{1}$ Keynes' research objective was to explain why a market-based economy could lead to a significant under-utilisation of resources, as occurred in the Great Depression, and to identify policies that could ameliorate such an event. Keynes' theoretical contributions were bolstered by the publication of national accounts data for the first time in the 1940 s. $^{2}$ The development of national accounts data meant that economists

\footnotetext{
${ }^{1}$ Keynes (1936); see also a shorter summary in Keynes (1937).

${ }^{2}$ The development of national accounts data was supported in the 1930s by the work of Colin Clark in the United Kingdom and by Simon Kuznets at the National Bureau of Economic Research in the United States.
} 
could begin to explicitly characterise how interest rates, output, employment, and inflation related to each other.

Keynes posited relationships that directly linked aggregate variables together. ${ }^{3}$ Perhaps the most iconic aggregate relationship that Keynes specified was the consumption function, relating current aggregate consumption to current income and other variables such as the rate of interest. ${ }^{4}$ This relationship between aggregate income and aggregate consumption was not built from first-principles analysis of individuals' behaviour, but was seen as a 'fundamental psychological law' that might be 'relied on with great confidence'. Hicks (1937) formalized Keynes' General Theory with the IS-LM model that most of us are familiar with from undergraduate economic degrees. The IS-LM model is static and does not fully specify the dynamic evolution of macroeconomic variables through time. ${ }^{5}$

Keynes' consumption function was soon subject to criticism. Underpinned by the macroeconomic facts brought to light by national accounts data, Friedman (1957) and Modigliani and Brumberg (1954) both took issue with the function that Keynes had specified. Rather than focus on a contemporaneous relationship between income and consumption, Friedman and Modigliani and Brumberg developed lifetime conceptions of consumer behaviour. Friedman developed the permanent income hypothesis, noting that permanent and transitory changes in income likely have differing impacts on consumption, and Modigliani and Brumberg developed the life-cycle hypothesis. Both theories imply that decision-makers need to take future circumstances into consideration when making decisions in the present. Expectations are therefore crucial for consumer decision-making.

The Keynesian orthodoxy broke down over the late 1960s and 1970s. Mankiw (1990) argues that Keynesian modelling was assailed by both empiri-

\footnotetext{
${ }^{3}$ Romer (1996) describes (traditional) Keynesian models as "directly specifying relationships among aggregate variables".

${ }^{4}$ Keynes (1937) asks "what governs the amount of consumption-expenditure?" And answers that "[i]t depends mainly on the level of income." Keynes (p. 220) considered this relationship 'absolutely fundamental' to the theory of effective demand set out in the General Theory.

${ }^{5} \mathrm{~A}$ typical theoretical exercise for the IS-LM model would be to specify a change in a parameter and then employ a comparative statics analysis, describing the new equilibrium that the economy would ultimately arrive at, though without formally describing how variables would transition to that new equilibrium, i.e. without specifying the dynamics.
} 
cal and theoretical challenges, and that the confluence of these two challenges substantially eroded faith in Keynesian macro modelling. Part of the theoretical challenge arose in the late 1960s. Friedman (1968) and Phelps (1968) both attacked the Phillips curve that had been tacked on to the IS-LM model to explain price dynamics. They argued that the inflation-output 'tradeoff' embodied in the Phillips curve ${ }^{6}$ was not sustainable in the long run, and that efforts to maintain low unemployment levels and high levels of output through monetary stimulus would simply result in higher rates of inflation. The inflation outcomes of the 1970s seemed to confirm Friedman and Phelps' hypothesis. Traditional Keynesian models could not explain these high rates of inflation.

Another theoretical challenge arose with respect to expectations. Lucas, Sargent, and others introduced rational expectations into macro-models, following on from Muth $(1960,1961)$. Rational expectations of future outcomes depend on i) what is known about the current state of the world; and ii) on the decision-maker's understanding of the dynamics of the economy. With rational expectations the 'understood dynamics' are consistent with how the economy actually behaves. Rational expectations does not imply perfect foresight - since shocks will occur in future that cannot be perfectly anticipated but it does imply that decision-makers [eventually] understand how the economy operates, they forecast in a manner consistent with the data, and they do not make systematic forecasting errors. If expectations of the economy are at odds with the dynamics of the economy, if expectations are not rational, then data should yield surprising outcomes, prompting decision-makers to alter their forecasting processes and behaviour.

The assumption of rational expectations has important implications for model dynamics and more particularly for the ability of policy to affect real macroeconomic outcomes. In an influential paper, Sargent and Wallace (1975) showed that systematic monetary policy was irrelevant for the cyclical variation of output and unemployment when rational expectations were introduced into a then-conventional macro model. Sargent and Wal-

\footnotetext{
${ }^{6}$ See Phillips (1958). Note that the 'tradeoff' perspective is associated with Samuelson and Solow (1960), and become embedded in Samuelson (1961). See the discussion in Sleeman (2011) and Leeson (1997).
} 
lace's analysis implied that stabilising nominal variables, such as inflation with no feedback from the economy to policy settings - was the appropriate way to implement monetary policy. A more nuanced interpretation of the paper is that private agents' ability to infer the structure of the economy and to alter the way in which they form their expectations can have important implications for the consequences of public policies.

Subsequently, it was realised that rational expectations can be compatible with monetary policy affecting output and real variables. Fischer (1977) and Taylor (1979) showed that monetary policy could have real effects if there were nominal (e.g. wage or price) contracts that lasted several periods even though they assumed expectations were rational. So, there remained, after all, a role for monetary policy in stabilizing real activity, and nominal rigidities were found to be central to this role. In such models the way in which firms set prices is at the heart of monetary policy's ability to affect the real economy. A central policy question is: if monetary authorities stimulate aggregate demand will firms actually choose to produce more and employ more people? Obviously if supply (quantity) decisions are determined ex ante then changes in demand will not impact quantity supplied. But if suppliers set prices and adjust quantities then a change in demand will lead to changes in the amount produced. (Lester 1946, 1947, Machlup 1947a,b and Stigler 1947 provide an early debate on marginalism and the factors underpinning changes in prices, output, and labour demand. Christiano et al. 2018 discuss how DSGE models typically embed acyclical movements in marginal cost so that firms satisfy movements in demand.)

Taylor and Fischer rehabilitated the traditional Keynesian conclusion that policy can stabilize the economy. ${ }^{7}$ But Lucas (1976) nevertheless showed that the methodology used to assess policy implications was materially affected by the assumption of rational expectations.

"Lucas pointed out that most policy interventions change the way individuals form expectations about the future. Yet the proxies for expectations used in the macroeconometric models

\footnotetext{
${ }^{7}$ See Mankiw and Romer (1991b,a) for a collection of 1970s and 1980s journal articles that largely define the New Keynesian research agenda, emphasising nominal and real rigidities, imperfect competition and coordination failures.
} 
[of the 1960 and 1970s] failed to take account of this change in expectation formation. Lucas concluded, therefore, that these models should not be used to evaluate the impact of alternative policies." Mankiw (1990, p. 1647).

Another way of thinking about this issue is to realise that private sector decisions strategically interact with public policies: optimal choices for private individuals depend on what public policies are implemented. If public policies change then the optimal consumption and investment decisions of private individuals may also change, altering macro dynamics. Conversely, people's preferences - their ranking of different alternatives - are conventionally assumed to be invariant to public policies, and thus provide a robust starting point from which to build models of human behaviour.

Rational expectations has become pervasive in macroeconomic modelling. One reason for making this assumption is because it is believed that in the long run people will understand how the economy behaves and they will not make systematic errors. If decision-makers' expectations are not rational then implicitly - possibly even explicitly - they are making forecasting mistakes that imply they are 'leaving money on the table' that could be used to improve their own welfare if they behaved differently.

In an insightful monograph discussing the limits of rational expectations, Pesaran (1987) argues that it is not obvious that agents can ever learn the 'true' model of the economy from their own experience, particularly when different individuals have different information sets. The rational action for a household or firm depends not only on public policies but also on the private 'policies' of private agents. A large literature has subsequently developed investigating the interplay between learning and expectations and 'sticky information', exemplified by Evans and Honkapohja (2010) and Mankiw and Reis (2002, 2010). See also the conclusion of Binder and Pesaran (1995). Notwithstanding this literature, the bulk of macroeconomic models developed to understand business cycle and monetary phenomena adopt rational expectations and common knowledge as working assumptions. One problem with the assumption of rational expectations is that in flexible price models agents respond promptly to new information. Empirically, however, adjustment processes appear to be more protracted and prolonged. Various 
modelling devices, such as introducing habit into consumer preferences and investment adjustment costs, have been introduced to more closely matched the dynamics observed empirically.

\subsubsection{Empirical modelling during the 1960s and 1970s}

Empirical macro models seek to explain the joint probability distribution of variables such as output, inflation, interest rates, and unemployment. But macro variables are stochastic processes, sequences of random variables ordered temporally. Thus, the focus of empirical macro models, particularly those used for monetary and fiscal policy purposes, is on dynamics: macro models seek to explain how variables at different points in time relate to each other.

As Diebold (1998) describes, macro modelling in the 1960s and 1970s sought to identify and estimate "systems of stochastic difference equations designed to approximate the postulated decision rules of Keynesian macroeconomic theory". Empirical macro modelling was explicitly focussed on dynamics, though theory at the time was largely silent on this issue.

Empirical models in this period were large, complicated beasts, sometimes with hundreds of equations, possibly thousands of parameters, and huge numbers of restrictions (e.g. many variables were absent from some of the equations). If you wanted to know about a variable then chances are these models would contain an equation for it. Fromm et al. (1972), for example, report simulations from the Brookings model, which contained equations for 'agriculture, forestry and fisheries' and also 'farming', regulated industries (such as railroad and non-railroad transportation, communications, and public utilities), personal consumption on food and beverages, and many more sectors besides.

But all was not well with these large-scale, empirical macro models. In particular, three major criticisms developed. First, as new data arrived in the 1970s the parameters of the equations seemed to be subject to instability (see for example Fromm et al. 1972, p. 201). There is, for example, a voluminous literature about the instability of just one of these equations - money demand. Judd and Scadding (1982) and Goldfeld and Sichel (1990) provide 
surveys and Lütkepohl (1994) provides a more recent contribution. Sims (1980) showed that equations from large-scale macro models were unstable and argued that the instability related to the ad hoc nature of restrictions embedded in the equations.

A second problem with these models became evident as econometricians began to better understand the properties of integrated variables (variables that need to be differenced to have a stable mean). Econometricians realised that regressions with unrelated integrated variables would lead to spurious parameter estimates - one could conclude that two series were strongly related to each other even though in truth they were not - which would also adversely affect the predictive content of such relationships. The spurious regression problem for trending variables was first noted by Granger and Newbold (1974), with Phillips (1986) developing an asymptotic theory for the parameters and significance tests of such regressions. A large literature then developed to test for cointegration relationships, including Engle and Granger (1987), Stock and Watson (1988), Johansen (1988), Phillips and Ouliaris (1990), and many others.

The third and equally damaging finding was that simple statistical models such as univariate autoregressions (where $X_{t}$ is explained using only $X_{t-1}$, $X_{t-2}$, etc.) could provide better forecasts than the complicated large-scale macro models (see e.g. Nelson 1972). Given the relative costs of constructing univariate time series models and large-scale macro models, it was no longer clear that the latter were worth the effort.

This is the jumping off point for the methodological discussion that we turn to next. The methodology initiated by Kydland and Prescott (1982) has been the dominant approach to business cycle fluctuations for the last three decades.

\subsubsection{Real business cycle and New Keynesian DSGE models}

Kydland and Prescott sought to explain business cycle fluctuations by taking a neoclassical growth model and augmenting it with a single (technology) shock. Methodologically, they argued that models should be built by spec- 
ifying people's objectives and the constraints they face, and that rational expectations should be used to characterise people's views about the future. (For more discussion see Karagedikli et al. 2010.)

With the addition of maximization, this framework can be used to determine how people behave, by looking at the corresponding first order conditions. When agents maximize their objectives, outcomes are as good as they can possibly get, and there is no incentive for decision-makers to alter their behaviour to achieve a better outcome, where 'better' is judged in some expected utility sense. There is in some sense an equilibrium between the way people form expectations and the dynamics of the economy.

In business cycle analysis we focus on choices associated with multiple time periods, and agents derive utility or profits in all periods. Because decision-makers are explicitly looking forward into the future their expectations are crucial ingredients of their current behaviour. The importance of stabilizing forward-looking expectations is one of the important insights that we obtain from DSGE models.

Modern macro models apply Alfred Marshall's marginalism, equating marginal benefits and costs, to a dynamic setting with multiple periods. Euler equations are the dynamic first order conditions that economic agents must satisfy if they are to maximize lifetime utility. In essence, people optimize their consumption, production, and labour supply decisions at adjacent points in time. Why consume today when you could save and consume tomorrow? Why work tomorrow to get a unit of consumption tomorrow if you could work today and save for tomorrow instead? The marginal costs and benefits of all these variables, in utility terms, should be equal.

The analysis of New Keynesian DSGE and real business cycle (RBC) models is quite different to the traditional Keynesian macro models that specified direct relationships between macroeconomic variables and did not worry too greatly about articulating agents' objectives or constraints. Macro variables within RBC and DSGE models are inter-related, through constraints and through objective functions, but not necessarily as directly as in Keynesian models. This point is taken up in more detail below.

For a consumer, lifetime income represents a major constraint for consumption decisions. In RBC/DSGE modelling the consumer's lifetime bud- 
get constraint is broken up into a sequence of constraints that connect wealth at time $t$ to wealth at time $t+1$. (Starting wealth plus income less expenditure equals wealth in the next period.) If these constraints are summed together then we have the property that lifetime expenditure and income must be equal. While we do not explicitly see lifetime income or Friedman's 'permanent income' in DSGE models, the lifetime budget constraint still binds. For a firm the production function is the major constraint (without the constraint of a production function a firm would maximize profit by producing an infinite amount). Period-by-period optimisation decisions also guide firms' pricing and investment decisions, since both decisions embody intertemporal tradeoffs.

Consumers' and firms' intertemporal optimisation results in a system of nonlinear Euler equations. These equations connect choice variables at time $t$ and $t+1$, ensuring that marginal benefits (or marginal costs) at adjacent points in time are equal as per the examples above. Analogous first order conditions apply at all points in time, e.g. for $t+1$ and $t+2$. Thus in these models decision-makers' choices for consumption and labour supply are optimized for all pairs of time, time $t$ and $t+2$; time $t$ and $t+3$, time $t$ and $t+4$; and so on.

Nonlinear Euler equations also implicitly connect our endogenous choice variables to exogenous variables and to a vector of 'state' variables which summarise the 'current location' of the economy. Actual outcomes are subject to risk/uncertainty because of the shocks that buffet the economy. Stochastic shocks mean that we cannot know exactly what will occur in future periods. Decision-makers must optimize their choices now against the expected array of future outcomes, where these future outcomes are assessed by looking at utilities and probabilities.

Nonlinear systems of equations are hard to solve and hard to understand. Typically, we approximate the nonlinear 'systems of equations' by linearising around the steady state (using perturbation methods), because solving linear systems of equations is more straightforward. Approximating the equations trades tractability for accuracy. If the non-linearity is important then the linearised approximation may not do a good job of capturing dynamics. More particularly, the linearisation is unlikely to be very accurate if we are 'far' 
from the steady state.

Projection methods provide an alternative approach to characterise global descriptions of dynamics, as opposed to the local-to-steady-state solutions provided by perturbation approximations. But projection techniques are computationally intensive and they place severe constraints on the size of models that can be explored. And these models are typically too small to be useful as empirically-oriented models providing forecasting and policy advice. See Judd (1999), DeJong and Dave (2007) and Heer and Maußner (2009) for discussions about these computational techniques.

The linearised first order conditions that are obtained from optimizing the model have expectation terms embedded in them. To be able to use the model for forecasting we need to determine what the expectations are as functions of the exogenous variables. We can then transform the 'expectational difference equations' into a reduced form analogous to a vector autoregression. In the reduced form, future outcomes are solely a function of past, realised variables, and it is then easy to determine forecasts using the recursive structure of the model.

Backward looking expectations are directly a function of past, realised observations and it is therefore easy to ascertain what such expectations should be conditional on past data. Rational expectations, by way of contrast, imply that people are forward-looking, and they use the model structure to compute their forward-looking expectations. Any change to the model structure will also change how expectations are formed.

Rational expectations imply restrictions that we hope will sharpen the parameter estimates of our models, improving their performance. Parameters in reduced form models are combinations of the structural parameters from our first order conditions, where structural parameters are those that "particularise' preferences or constraints. (In chapter 2 we refer to a particular parameterisation as an 'instantiation', borrowing a label from computer science.) The expectational difference equations (the Euler equations) restrict admissible parameters of the reduced form.

Applying restrictions that are true - such as excluding variables from an equation that should be excluded - assists in the estimation of parameters. Because macro models are not estimated on experimental data, regressors 
(the left hand side variables) are typically correlated. If we wrongly include a variable on the left hand side of an estimated equation then the covariance of this included variable with other, correctly-included regressors will inflate uncertainty about parameter values (parameter estimates will have larger confidence intervals). Conversely, omitting a variable that belongs in the regression will bias parameter estimates if the regressors are correlated.

It is worth emphasizing this point more starkly: imposing 'good structure' (correct restrictions) is good since it will result in more accurate parameter estimates, but imposing 'bad structure' is bad since it will likely bias parameter estimates. To reiterate: rational expectations introduce restrictions that we hope will sharpen the parameter estimates of our models, improving their empirical performance and also enabling modellers to understand how expectations processes will change as structural features of the model change - features such as preferences, constraints, and public policies.

\subsubsection{An identification detour}

At this point it is worth taking a slight detour to discuss parameter identification, and why parameter restrictions are important for identification. Parameter identification is about our ability to infer the parameters that generated the data from the data that are actually observed. A model's parameterisation determines all of the properties of the variables in the model, including any transformations of the variables, and is thus crucial for any inferences that we might wish to draw from the model.

The simplest econometric example of identification is a situation with supply and demand curves where we are interested in understanding the parameters of the two curves. Suppose that we observe prices and quantities, points 1, 2, and 3 in panel (a) of figure 1.1. What does this price and quantity information tell us about the shape of the underlying demand and supply curves?

The answer to this question is that we know nothing about the underlying supply and demand curves unless we have additional information or make additional assumptions. If we know that some shift variable has shunted the demand curve but the supply curve has not changed - a restriction on 
Figure 1.1: Identifying supply and demand curves

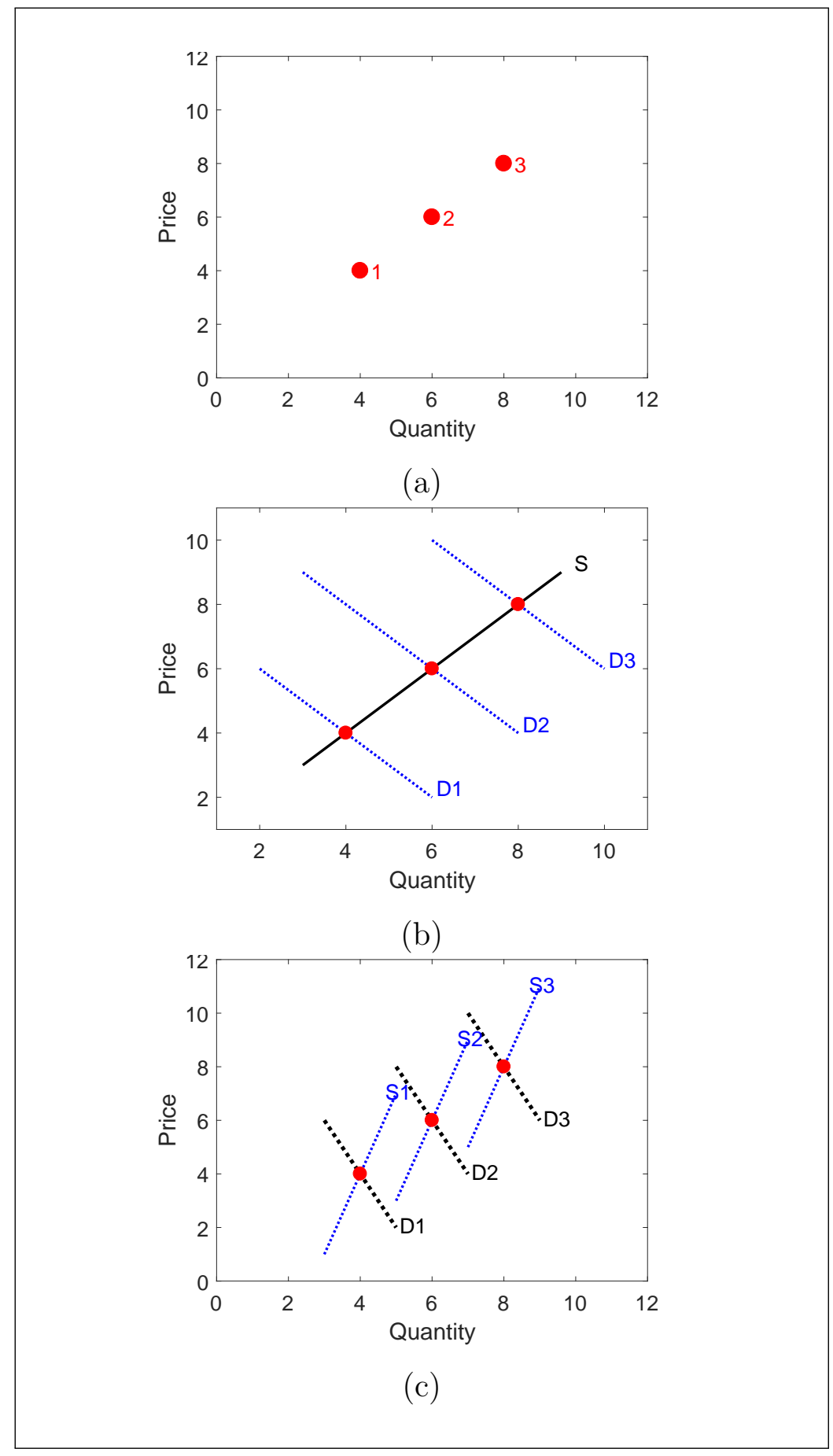

Source: Greene (1997). 
a parameter, the shift-variable has a zero coefficient in the supply curve then the three points trace out the supply curve, e.g. panel (b). But if the shift variable has moved both supply and demand simultaneously, as in panel (c), then the three points will not trace out either the supply curve or the demand curve. It is also important to realize that the situation in panel (b) does not tell us anything about the slope of the demand curve. Parameter restrictions are crucial to identify the shape of these functions if supply is unaffected by some exogenous variable, then it has a coefficient of zero. This restriction suffices to identify the shape of the supply curve, but additional restrictions will be required to identify the slope of the demand curve. Systems of simultaneous equations face this same identification problem, and require restrictions to identify the parameters of the system. Specifying appropriate restrictions is one of the big challenges of macro modelling. Identification is an issue both for Keynesian models and for the real business cycle (RBC) and DSGE models that are described shortly.

We provide a figurative description of the identification problem in figure 1.2. A model parameterisation determines the probability distribution and hence variances and covariances of the data. The identification problem is to ascertain whether there is an inverse mapping, illustrated with the dashed (red) line, that correctly and uniquely maps from the data moments back to the parameter vector that generated the data. The dotted (white) line around $\theta_{3}$ represents the fact that our identification analysis is 'local' to a particular point. The identification technology determines whether another parameter vector in the neighbourhood of $\theta_{3}$ could have generated the observed data moments. It is worth emphasising again that identification is the product of model specification (theory) and data. Changing either theory or data could change the identification properties of the model.

\subsubsection{Model properties}

Modern business cycle analysis is primarily concerned with understanding the propagation of independent 'shocks', and about understanding how these shocks alter endogenous variables (reflecting people's decisions) and observed macroeconomic variables. From a technical perspective, impulse response 
Figure 1.2: Figurative illustration of the identification problem

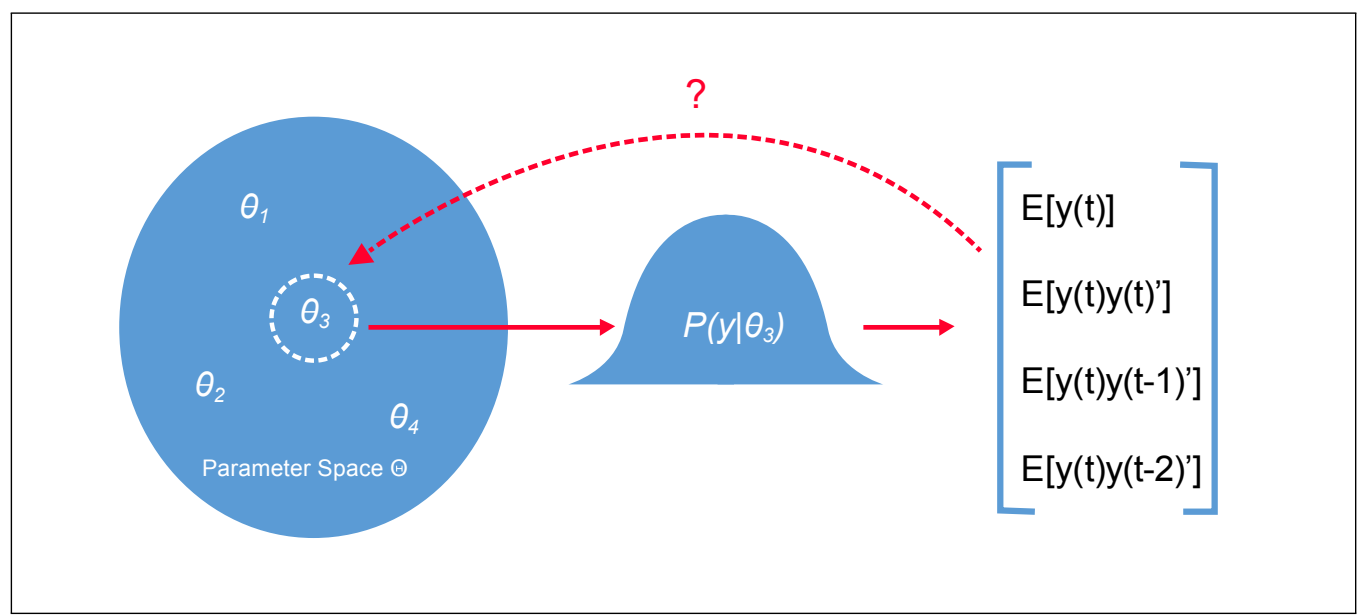

functions trace out how individual structural shocks propagate to all of the observed variables. Naturally, shocks occur at each point in time. The model and our estimates of parameters enable us to infer or estimate values for these unobserved shocks.

The first order conditions associated with constrained intertemporal optimization enable us to trace out how endogenous variables evolve in response to shocks; these shocks are the exogenous variables of the model. The exogenous variables are typically assumed to be uncorrelated, so it is possible to consider thought experiments where just a single shock is changed at a time.

If two shocks are correlated then it is probabilistically unusual for one shock to jump without the other doing so simultaneously. If shocks covary it becomes difficult to separately account for the shocks' relative importance. This difficulty is particularly prominent if we are trying to decompose the variance of endogenous variables, since part of the variance would depend on the shocks' covariance term - how would we label that covariance contribution? Remember from elementary statistics that if $\epsilon_{1}$ and $\epsilon_{2}$ are random variables and $a$ and $b$ are constants then $\operatorname{var}\left(a \epsilon_{1}+b \epsilon_{2}\right)=$ $a^{2} \times \operatorname{var}\left(\epsilon_{1}\right)+b^{2} \times \operatorname{var}\left(\epsilon_{2}\right)+2 a b \times \operatorname{cov}\left(\epsilon_{1}, \epsilon_{2}\right)$. If $\epsilon_{1}$ and $\epsilon_{2}$ have zero covariance then it is straightforward to decompose the aggregate variation into variance components from $\epsilon_{1}$ and $\epsilon_{2}$. Ramey (2016), in her Macroeconomics Handbook chapter on the propagation of shocks, argues that the shocks should be 
"primitive exogenous forces that are uncorrelated with each other" (emphasis in original). Cúrdia and Reis (2010) relax this restriction and allow for contemporaneous and dynamic correlation amongst the shocks, which they find resolves some of the disparities between structural and reduced form models. For the most part, however, models with cross-correlated 'structural' shocks are a rarity.

In general, it is not straightforward to specify how one endogenous variable affects another. Imagine that we observe a unit increase in the exchange rate. What will happen to future output growth? The correct answer is "it depends". How output evolves depends on the exogenous shock that increased the exchange rate in the first place. Shocks are not created equal. DSGE models provide a lens that can be used to identify the underlying exogenous drivers, ascertain their effects, which we represent with impulse response functions, and determine their contribution to cyclical volatility, via forecast error variance decompositions. These are the analytical building blocks that are used intensively in the remainder of this dissertation.

\subsubsection{Real business cycle and New Keynesian DSGE models}

The three substantive chapters of this dissertation use dynamic stochastic general equilibrium models to address various analytical questions. This section spends a little time talking about the features of these models. Real business cycle and New Keynesian DSGE models are methodologically similar in that they build models from preferences and constraints. Typically, these models also assume maximizing behaviour, rational expectations and market equilibria. Early-generation RBC models assumed that prices were flexible and focused on the role of technology shocks in driving cyclical fluctuations (see Hartley et al. 1998, ch. 1). In contrast, New Keynesian DSGE modellers have typically assumed that there are important nominal and real rigidities, and many types of shocks. The dividing line between these two approaches is beginning to blur, with some flexible-price RBC models incorporating additional shocks and frictions, as in chapter 3 .

Keynesians, new and old, tend to believe that fiscal and monetary policy 
can play an important role in stabilising the economy. New Keynesians typically incorporate features in their models to replicate this property, ensuring that there is a substantive, welfare-enhancing role for policy. ${ }^{8}$ But to justify this position they have to assume that there are nominal rigidities (eg wage and price stickiness) or other frictions whose effects can be offset with policy. RBC modellers, on the other hand prefer to start from a very simple model, to see how much can be explained with as few elements as possible. One can interpret the RBC agenda as providing a well-understood baseline from which to consider more complicated DSGE models.

Parsimony is a virtue in modelling, as simple models are easier to understand. It is also usually harder to estimate parameters for larger models accurately. If we introduce additional complexity into our models we need to have a good rationale for doing so. Does the additional complexity change our understanding of the dynamics of the economy? Does such complexity help us to fit the data better, forecast better, or enable us to explain additional data? Does additional complexity change our views on optimal policy? These are important challenges for more complex models.

\subsubsection{The empirical performance of macro models}

From a research perspective, RBC and New Keynesian dynamic stochastic general equilibrium models provide a playing field in which to explore different assumptions. The main dimensions that DSGE researchers explore are the specification of preferences, the specification of constraints, and the impact of particular frictions.

If DSGE models are to be credible empirical models that can be used for forecasting then they need to overcome the earlier criticism of large-scale macro models. That is, they need to demonstrate that they can forecast as well as simple time series models, such as univariate autoregressions. Parameters must also be stable or at least predictable. If the models do not do a reasonable job of representing the data it is hard to take their quantitative policy recommendations seriously. Furthermore, the policy conclusions that

\footnotetext{
${ }^{8}$ Minsky (1975, p. 10) observed "the primary policy message of Keynes - that slumps are unnecessary and a waste of both human and nonhuman resources - has become a fundamental political axiom guiding economic policy."
} 
are provided by DSGE models depend on their exact parameterisation, see for example De Paoli (2009).

Estimating simultaneous equations models via maximum likelihood has proved challenging. ${ }^{9}$ Fortunately, Bayesian techniques have been developed that are easier to implement, and that enable both prior beliefs and likelihoods to influence parameter estimates, thereby increasing their policy relevance. Software and methods associated with Sims (2002), Schorfheide (2000), Del Negro and Schorfheide (2004), and Adjemian et al. (2011) have made the solution and estimation of DSGE models by Bayesian methods much more accessible.

In a couple of influential papers, Smets and Wouters $(2003,2007)$ argued that the forecasting performance of their DSGE models for Europe and the United States, with multiple shocks and multiple frictions, were competitive with time series forecasts. Smets and Wouters' forecasting success for the Euro Area and the United States, and the prospect of improved policy analysis, encouraged central bank modellers to develop similar models for Britain, Canada, Sweden, Norway and of course New Zealand. (See e.g. Bayoumi 2004; Murchison and Rennison 2006; Adolfson 2007; Brubakk et al. 2006; and Beneš et al. 2009.)

It is worth reiterating that RBC and DSGE models imply particular reduced forms once we have substituted out for expectations. To improve fit, however, one does not simply introduce additional regressors into the reduced form. Instead, the fit to the data is improved by introducing a variety of 'frictions' into DSGE models, either in the preferences (eg habit formation) or constraints (such as capital adjustment costs or nominal price rigidities). Christiano et al. (2005), for example, developed a DSGE model with staggered wage contracts and variable capital utilisation to improve the persistence of both inflation and output in response to monetary policy shocks. Additionally, the exogenous shocks are also assumed to have a degree of persistence, and so are typically modelled as being autoregressive processes, ie $e_{t}=\rho e_{t-1}+\epsilon_{t}$, where $e_{t}$ is the exogenous shock, and $\epsilon_{t}$ is white noise. These frictions and generalisations to the shock processes are either 'dirty tricks' or

\footnotetext{
${ }^{9}$ Relatively few macroeconomists estimate DSGE models using maximum likelihood methods. Though see Ireland (2004a,b) for the exception to this statement.
} 
'pragmatic amendments', depending on your modelling preferences. These features often make an important contribution to the dynamics of the model. Consequently, there will always be legitimate questions about the extent to which DSGE models are genuinely 'structural'.

\subsection{Criticism of the DSGE framework}

While this dissertation is based on DSGE models it is worth taking a moment to dwell on some of the criticisms that have been levelled against them. First, the models rely on infinitely-lived agents and heterogeneity amongst agents is usually quite limited. This lack of heterogeneity is problematic when financial transactions are involved, since borrowers of the same type will not choose to lend to each other. ${ }^{10}$ Stiglitz (2018) notes that the housing market was central to the 'great recession' in the United States, 2007-2009, and suggests that DSGE models of housing and their finance miss important life-cycle heterogeneity. The lack of heterogeneity also means that agents have the same information and beliefs about the economy.

Second, the microeconomic foundations of agent decision-making in DSGE models is also subject to criticism. DSGE models invariably rely on the assumption of rational expectations and assume that agents maximise their objective functions subject to constraints. Yet a considerable literature on behavioural economics (see for example Altman 2006 and Cartwright 2011) and experimental economics (see Kagel and Roth 2016a; Plott and Smith 2008; Kagel and Roth 2016b) argues that there are important cognitive limitations, which call into question a central building block of DSGE models. Yet another literature focuses on the role of learning (exemplified by Evans and Honkapohja 2010). In the spirit of the latter, Eusepi and Preston (2011) consider how learning may disconnect expectations from fundamentals and thus how shifts in beliefs can result in business cycle fluctuations. Grauwe (2010) argues that learning can generate endogenous cycles, reflecting bouts of optimism or pessimism, and that learning provides an intrinsic mechanism to generate the persistence seen in output and prices. See also Slobodyan and

\footnotetext{
${ }^{10}$ We will see in chapter 4 a model with two types of agents to introduce a motive for lending.
} 
Wouters (2012), who use the Kalman filter applied to small forecasting models to generate expectations. Learning of this type reduces the importance of wage and price mark-up shocks as a source of business cycle fluctuations.

Stiglitz (2018) trenchantly argues that DSGE models have many substantial failings, including the fact that they fail to provide convincing explanations of the large declines in output and employment that occurred during the global financial crisis, not to mention the slow recoveries that then ensued. Stiglitz argues that the methodology that is used to estimate models fails to target these extreme downturns, even though such down-turns are central to the design of policy. In response to this criticism, Christiano et al. (2018) note that modern DSGE models are estimated using Bayesian techniques that maximise marginal posterior densities. Implicitly, these techniques can be thought of as targeting a weighted average of empirical moments, though it is true that they do not necessarily target the moments that Stiglitz thinks are of pre-eminent interest. Another response to this criticism is that monetary and fiscal policies needs to be conducted in good times and in bad, and thus matching the properties of non-crisis periods is still important if DSGE models are to inform policy.

Stiglitz also notes that the equilibrium nature of DSGE models rules out coordination problems - the price mechanism functions miraculously and comparatively little attention is paid to how transactions are implemented, including the information asymmetries and cognitive limitations of the agents that are transacting. The search and matching models of the "new monetarist economics' take these frictions and market details seriously, and provides a more deeply micro-founded perspective on the nature of finance. For an overview, see Williamson and Wright (2010, 2011) and Wright (2018).

Stiglitz also criticises the modelling of uncertainty in DSGE models. Typically uncertainty is represented as quantifiable risk - appropriate probabililty distributions can be inferred for the stochastic elements of the model, and in many instances these risks can be diversified using financial markets. Such assumptions are typically made for reasons of tractability. If households and firms do not know what they do not know, or cannot characterise the probabilities of uncertain outcomes then it becomes problematic to infer what actions such agents will undertake given their present circumstances. 
In 2010 five economists presented testimony to the Committee on Science and Technology of the US Congress. The hearing of this session of the committee $^{11}$ considered 'building a science of economics for the real world', and the allocation of national science foundation awards to macroeconomics. Expert witness testimony was presented by Professors Solow, Winter, Page, Chari, and Colander. The expert testimony provides a cross-section of views, some supportive of DSGE modelling and others considerably more negative. A common theme was the need for a greater degree of pluralism in the types of models being funded, developed, and applied (see for example the testimony by Page and Colander and, separately, Wren-Lewis 2018). Some criticism, such as Solow's perspective on the modelling of labour markets in DSGE models, seems mis-placed. Solow asserts that DSGE models have no place for involuntary unemployment. Yet the DSGE literature now incorporates, for example, search-and-matching frictions that provide a framework to analyse unemployment. Returning to information asymmetries, Sidney Winter in his written testimony emphasised the information asymmetries and institutional details that were embedded in the securitisation of housing loans. It is a reasonably fair conclusion that these details are typically omitted from most DSGE models. Conversely, 'new monetarist' models often embody search and matching frictions and pay much greater attention to such features.

Winter (see p. 24 of the record of the hearing) goes on to claim that "the DSGE model does not contain even a rudimentary representation of the financial sector at the level of the "IS-LM" model". While such a claim is true of many DSGE models, it was not universally supportable even in 2010 when the claim was made, and is less true eight years later. To note just a few examples, Bernanke et al. (1996) develop a model of financial markets that incorporates an external finance premium that arises from a costly state verification problem, and Galí et al. (2007) investigate consumer liquidity constraints. Gerali et al. (2010) study the role of credit supply factors in a model with financial frictions and an imperfectly competitive banking sector. Gertler and Karadi (2011) examine unconventional monetary policy in a model with financial intermediaries. Hollmayr and Kuhl (2016)

\footnotetext{
${ }^{11}$ One hundred eleventh congress, second session, July 2010, Serial Number 111-106.
} 
examine imperfect information about financial frictions in a DSGE model with a banking sector with a limited enforcement problem. And Jacob and Munro (2018) examine macro-prudential policies in an open economy DSGE model with a banking sector. Chapter 4 of this dissertation contributes to this literature, incorporating a simple financial friction and illustrating how such a friction affects the relative importance of different structural shocks.

Yet another criticism is that DSGE models are often solved using first order, linear perturbation techniques. The dynamics of the system are only well-approximated near the steady-state, and cannot be used to describe tail events. More generally, linear techniques - by definition - cannot capture non-linearities that again may be important for tail events. The macroeconomics profession is beginning to move on from purely linear approximations of the equations in structural models. In some cases higher order approximations are required, eg to answer questions about welfare and optimal policy (see for example Woodford 2003, Heer and Maußner 2009, SchmittGrohé and Uribe 2004, and Kim et al. 2008). Projection methods are also becoming increasingly popular, but at a computational cost that limits the complexity of the models.

Christiano et al. (2018) provide additional responses to some of the criticisms levelled by Stiglitz and others. They note that the family of DSGE models includes efforts to model financial frictions, heterogeneity, deviations from traditional rational expectations, such as k-level thinking and various forms of learning, and efforts are now being made to relax the assumption of common knowledge.

Let me conclude this section with the following observation. DSGE models, like all models, are imperfect representations of the macroeconomy. However, they provide a transparent platform that can be used to model important macroeconomic issues, they capture important mechanisms and constraints, and they continue to evolve and adapt to new empirics and new theory. 


\subsection{Introducing three chapters}

Having provided a potted history of macro modelling and the constituent elements of dynamic stochastic general equilibrium models, let us now turn to a brief description of the following chapters. The next chapter explores whether it is possible to identify - in an econometric sense - the parameters of a DSGE model. Econometric estimates of parameters determine the volatility and persistence of different shocks and determine the contribution of these shocks to aggregate variation. Chapter 3 explores the consequences of a new candidate shock, a migration shock, and quantifies its importance for business cycle fluctuations. The final chapter investigates the consequence of a friction for the contribution made by investment-specific technology shocks. The three chapters are unified by their use of DSGE models. The latter two chapters identify the contribution of independent exogenous drivers, making use of impulse responses and forecast error variance decompositions to inform the analysis. The literature on financial frictions also illustrates that the choice of data matters for inferences, tying back to the focus of chapter 2 .

\subsubsection{Foreign data are not necessary for identification}

Chapter 2 takes an open economy macro model for a small open economy and addresses three questions:

1. What data series should be used to identify the parameters of such a model?

2. Are foreign data important for the identification of domestic parameters? And lastly,

3. Which structural parameters are interdependent?

The chapter illustrates a practical methodology that enables an investigator to understand which data series are informative about parameters, based on Iskrev (2010b). When parameterised, models fully determine the properties of the variables in the model. The problem of identification is to establish whether an inverse mapping also exists, from observed data back into the parameter space of the model. We consider a situation in which 
the investigator must choose which series to use to inform data estimation, where the series will be some subset of the variables contained in the model. We make use of the inverse function theorem to evaluate whether an inverse exists. Given suitable conditions, a vector-valued function will have an inverse if the vector of partial derivatives with respect to the parameters has full rank. We evaluate the ranks of derivative matrices that are conditional on an initial parameter vector and on the data series chosen as observables. We use these rank matrices to determine combinations of observables that can be used to identify the model parameters. In principle, this identification analysis may depend on the underlying parameterisation, and so we explore identification at different points in the parameter space.

Using this methodology, we find that foreign data are not particularly useful for identification of domestic parameters. In the context of our model identification problems arise in the mapping from deep structural parameters through to the parameters of the expectational equations. Our methodology can easily ascertain when problems arise in this mapping. While the exact conclusions of this chapter are specific to the particular model that we examine, the methodology we illustrate can be easily applied to other models to ascertain whether they too suffer from similar identification problems.

The methodology deployed and extended in this dissertation can also be used to learn about the properties of a model. In particular, the methodology highlights which parameters are interdependent and which parameters are informed by observed data. We emphasise that the analysis examines the importance of foreign data for econometric identification and hence estimation and is not concerned with the contribution that foreign shocks make to domestic business cycle fluctuations.

Ultimately, identification of business cycle models matters because estimates of structural parameters are materially influenced by the data series that are used to inform the estimation (Guerron-Quintana, 2010). Since structural parameters determine both the specification of household preferences and the constraints that affect business cycle volatility, identification can be important for our understanding of household welfare. Curvature parameters in utility functions determine how (un)willing households are to vary their consumption and labour supply patterns, either across varieties 
of goods or through time. The parameterisation of constraints influences the degree and types of volatility extant in the economy, that households may seek to ameliorate. These welfare considerations have ramifications for the specification of monetary policy rules, and public policy choices more generally depending on the specification of the model.

Different policy-makers, different researchers or political authorities, may agree on the structure of a model but have different prior beliefs about how a model should be parameterised. The analysis presented in chapter 2 shows whether data will eventually dominate prior beliefs about parameters, determining whether analysis can - in the limit - resolve conflicting prior beliefs, usefully informing the design of policy rules.

\subsubsection{Skilled migration and business cycle dynamics}

Chapter 3 examines the business cycle effects that arise from an expansion of the population due to migration. Migration flows have become highly topical, even controversial, in countries such as New Zealand, Britain, the United States, Germany, and Scandinavia. While the debate on migration has become heated, comparatively little is known about the business cycle consequences of migration flows. This chapter contributes to the macroeconomic literature by illustrating the contribution that migration shocks make to cyclical fluctuations, and illustrates their dynamic impact. This chapter thus makes use of the archetypal tools of DSGE analysis that were described above.

The analysis presented here is conducted in per capita terms. Since the number of people living in a country inherently scales up its productive capacity and income, aggregate consumption and investment are naturally expected to increase. A more interesting question is whether these same quantities increase or decrease in per capita terms.

Chapter 3 specifies a dynamic stochastic general equilibrium model to explore the consequences of migration. Households consume foreign- and domestically-produced goods, and housing. Domestically produced goods are produced using effective labour and physical capital, where effective labour is a composite of physical labour and human capital. The level of human 
capital is particularly important for the dynamics of the migration shock. Human capital serves to amplify the labour supply impact of a migration impulse.

The model also embodies other frictions. Physical capital, for example, has a variable utilisation rate. Variable utilisation and capital adjustment costs are important for the dynamics of shocks. Variable utilisation provides an extra margin of adjustment in response to a shock, while capital adjustment costs serve to slow the propagation of shocks to investment.

The model has three stocks of capital: physical capital; housing; and human capital. A migration inflow erodes the per capita stock of physical and housing capital, but the effect on the stock of human capital is ambiguous because migrants may have more or less human capital than domestic residents. The stock of migrant human capital relative to local resident human capital has a material impact on the dynamics of the migration impulse and on the contribution that migrant shocks make to business cycle fluctuations. In our baseline, we assume that migrants to New Zealand have 1.85 times as much human capital as local residents, based on analysis by Boubtane et al. (2016).

Using the estimated dynamic stochastic general equilibrium model of a small open economy, the chapter shows that migration shocks account for a considerable portion of the variability of per capita gross domestic product (GDP). While migration shocks matter for the capital investment and consumption components of per capita GDP, there are other drivers of cyclical fluctuations in these aggregates that are even more important.

Migration shocks are also important for residential investment and real house prices, but other shocks play a larger role in driving housing market volatility. In the DSGE model, the level of human capital possessed by migrants relative to that of locals materially affects the business cycle impact of migration. The impact of migration shocks is larger when migrants have substantially different - larger or smaller - levels of human capital relative to locals. When the average migrant has higher levels of human capital than locals, as seems to be common in most OECD economies, a migration shock has an expansionary effect on per capita GDP and its components, which also accords with the evidence from a restricted structural vector autoregression. 


\subsubsection{Financial frictions and investment-specific tech- nology shocks}

Chapter 4 rounds out the dissertation. This chapter investigates the contribution of investment-specific technology (IST) shocks in driving cyclical fluctuations in a closed economy model when a borrowing constraint is introduced into the model, à la Kiyotaki and Moore (1997). The constraint implies that borrowing must be collateralised by a certain amount of assets - typically physical assets such as capital goods or housing. Given suitable assumptions, collateralised debt arises as an optimal contract when the verification of the 'state' is costly (Townsend, 1979). Debt contracts are extremely prevalent, relative to what one might expected based on conventional general equilibrium theory. Townsend's paper on costly state verification was prompted by observations by Arrow (1974) and Radner (1968) that statecontingent contracts are only feasible if both contracting parties are able to verify the state. ${ }^{12}$ Financial frictions have also risen in prominence following the global financial crisis. The assumption that financial markets operate (approximately) frictionlessly has become much less tenable over the last decade. State-contingent verification is the canonical friction that we deploy in chapter 4.

Investment-specific technology shocks have been identified as a major driver of the business cycle, motivated by the ongoing decline in the relative price of investment goods. Greenwood et al. (2000), for example, suggests that investment-specific technological change is responsible for about 30 percent of the variation in US output. See also Fisher (2006), Justiniano et al. (2010, 2011), and Khan and Tsoukalas (2011). These shocks affect the rate at which investment goods are transformed into capital stock, and have been linked to frictions in financial markets, because financial intermediation is instrumental in facilitating investment. ${ }^{13}$ As noted in the chapter, parame-

\footnotetext{
${ }^{12}$ While Townsend's analysis is predicated on the implementation of such constraints by private lenders, constraints of this ilk have assumed greater prominence in the policy literature because macro-prudential authorities have co-opted them to employ 'loan-to-value' constraints on household borrowers purchasing houses, to try to limit the vulnerability of financial systems to down-turns in housing markets.

${ }^{13}$ For example, Justiniano et al. (2011) state that "investment shocks might proxy for more fundamental disturbances to the intermediation ability of the financial system."
} 
ter estimates and the variance contribution of these shocks depend on which data series are used to estimate the model, illustrating an important link between chapters 2 and 4 .

Chapter 4 asks whether the role of investment-specific technology is robust to the inclusion of the borrowing constraint. The short answer to this question is: 'No.' The style of analysis is similar to Carlstrom and Fuerst (1997) who examine the role of agency costs for business cycle dynamics. In contrast to Carlstrom and Fuerst, we estimate our model using the Bayesian estimation techniques that have become common over the last two decades.

A key feature of business cycles is the simultaneous co-movement of output, investment, consumption, and hours worked. In our model patient households fund borrowing by firms owned by impatient entrepreneurs, collateralised against physical capital. Investment-specific technology shocks increase the efficiency with which investment goods are produced from the factors of production. Such a shock reduces the price of investment goods. But the concomitant reduction in the price of existing investment goods tightens the collateral constraint, making it more difficult for entrepreneurs to borrow, and instead forcing them to reduce consumption to increase investment. Thus, the shock generates countervailing movements in consumption and investment, which is at odds with the positive correlation that is observed in the data. Other shocks must predominate to generate this positive co-movement. In the context of the model, shocks to the risk premium, which spontaneously increase interest rates affecting intertemporal substitution, supplant IST shocks as drivers of the business cycle. This chapter thus illustrates that the particular friction embedded in the model has a material impact on the relative importance of the shocks.

Before concluding, it is worth noting that this chapter deploys a particular financial friction - the collateral constraint - and other constraints or frictions are also worthy of investigation. Furthermore, the consequences of the frictions are likely to depend on exactly how the frictions are specified - just as investment adjustment costs have different implications to capital adjustment costs. The literature on financial frictions continues to build, and with it a greater understanding of which frictions fit the data better. This literature is too extensive to do much justice to here, having expanded 
rapidly since the global financial crisis. Examples include Gu et al. (2013), who discuss a rationale for bank intermediaries; Hall (2011, 2013), who looks at the intensification of financial frictions in a crisis; Brzoza-Brzezina et al. (2013), who compare a DSGE model with collateral constraints to a DSGE model with an external finance premium; Suh and Walker (2016) who take models with financial frictions to the data (and suggest that they do not necessarily improve fit relative to a model without frictions); Kolasa and Rubaszek (2015), who perform a similar exercise, concluding that a model with frictions in the housing market out-performs a model with frictions in the corporate sector; and Fuentes-Albero (2018), who examines the contribution of financial shocks, financial frictions, 'good luck', and 'good policy' in driving the great moderation. Many other papers, too numerous to mention here, explore related issues.

\subsection{Concluding remarks}

DSGE models provide a platform that can be used both to forecast the economy and evaluate the impact of different policies. The ability to evaluate policies stems from the fact that DSGE models are built up from representations of preferences that are assumed to be invariant to policies. Using DSGE models, it becomes possible to evaluate how people will modify their behaviour when public policies are changed, and therefore possible to determine what macro outcomes are likely to arise. Furthermore, because DSGE models characterise people's utility functions they can also be used to evaluate welfare, though any agent heterogeneity makes welfare evaluation problematic.

This introduction has described how DSGE models are built up from preferences and constraints, and how modellers amend DSGE models to better-fit the data. DSGE models also delineate the frictions or imperfections that provide a rationale for policy (such as nominal rigidities), and can be used to infer the primitive, fundamental drivers behind macroeconomic fluctuations.

All models are approximations to the world. DSGE models, for example, make use of assumptions that some people will find unpalatable (such as rational expectations and optimization). One benefit from modelling is that 
these assumptions are made transparently, and their impact can therefore be explored explicitly. Modelling is an evolutionary process that aims to identify 'better' models than the ones we currently employ. Models should be continuously tested and evaluated, and new alternatives should be considered as potential replacements. We should also refrain from discounting new models too hastily. Mankiw (1990) observes that Copernican helio-centric astronomy was initially inferior at predicting planet positions, relative to the Ptolemaic system in which planets circled the earth. But this empirical deficiency was overcome once it was realised that planet orbits were elliptical and not circular. 


\section{Chapter 2}

\section{Foreign data are not necessary for identification in a small open economy model}

\subsection{Introduction}

"Identification issues ought to be discussed in more detail in DSGE models, since they affect the conclusions we get from them." Fernández-Villaverde (2010, fn. 10)

"What econometricians can usefully do is to clarify what conclusions can and cannot logically be drawn given empirically relevant combinations of assumptions and data." Manski (2003, p. 12)

"A further step consists of preposterior analysis, which is concerned with the design of experiments. By studying the attributes of the posterior density prior to the observation... it is sometimes possible to compute the expected value of sample information and thereby to encompass data collection in the formal analysis." Drèze (1972, p. 8), emphasis added.

Which data series should be used to estimate a small open economy model? More specifically, are foreign data series useful for identification of domestic parameters? And which structural parameters are interdependent? 
In this chapter we apply and extend a simple methodology that answers these three questions. Our analysis illustrates that identification problems can creep into the mapping between 'deep' structural parameters and the parameters that specify the expectational equations that represent the model. The methodology that we develop can diagnose such identification problems and determine which parameters are subject to identification problems. While our answers to these questions are specific to our particular model, the methodology can easily be applied to similar models.

The parameters of business cycle models govern the properties of observed macroeconomic variables, their moments, impulse responses, forecasts and variance decompositions. Conversely, parameter estimates are determined by an inverse mapping from observed data back into the parameter space. This chapter focuses on this inverse mapping for a small open economy model.

Simulation methods associated with the Bayesian paradigm have become pre-eminently popular for estimating business cycle models. ${ }^{1}$ Consequently, we focus on parameter identification in the context of Bayesian estimation of a dynamic stochastic general equilibrium (DSGE) model. ${ }^{2}$ We concentrate on the full information methods that are commonly used to estimate DSGE models, though there is also a related literature that focuses on parameter identification for single equations. ${ }^{3}$

Identification is an important issue for Bayesians for two distinct reasons. First, identification analysis can determine which data series can be used to refine beliefs about parameters, and thus informs estimation. Second, identification analysis sharpens focus on the prior beliefs governing un-identified parameters. Identification analysis can show whether posterior inferences will ultimately be governed by the likelihood or whether prior beliefs will always play an important role in shaping beliefs. If the model is identified, we can eventually rely on the data to resolve disparate prior beliefs. In the con-

\footnotetext{
${ }^{1}$ An and Schorfheide (2007), Fernández-Villaverde (2010), Herbst and Schorfheide (2014), Fernández-Villaverde et al. (2016) and Herbst and Schorfheide (2015) discuss these methods. See also Robert and Casella (2004) for a more statistically-oriented treatment of simulation methods.

${ }^{2}$ Gabrielsen (1978) and Sims (1980, p. 2) provide elegant verbal definitions of identifiability.

${ }^{3}$ For examples of the latter, see Pesaran (1987), Nason and Smith (2008), Dufour et al. (2013), and Krogh (2015).
} 
trary case, other mechanisms will be required to resolve competing beliefs, such as voting, coin-tossing or paper-scissors-rock.

How forceful are our prior beliefs? Drèze (1972, p. 9) notes that classical exclusion restrictions are maintained even if they conflict with observations, while Bayesian priors are balanced against observations - via likelihoods - in proportion to their relative precisions. However, the balancing act between prior and likelihood may be somewhat illusory. 'Pre-posterior' identification analysis can highlight whether some Bayesian prior beliefs will always influence posterior beliefs. In a complementary approach, Müller (2012) evaluates the tradeoff between prior and likelihood by examining the sensitivity of posterior beliefs to shifts in the means of the prior distributions. However, Müller's approach rests on an unarticulated choice - a choice to use particular data series to estimate the model. More specifically, his analysis conditions on a specific data sample, determining the sensitivity of the means of the posterior to the priors. In contrast, we consider which data series might be useful for estimation before we have observed any particular sample.

In Bayesian analysis data $y$ are used to update prior beliefs about a model. But data are not manna from heaven that miraculously appear to feed the econometrician. They are instead the outcome of a mundane choice to collect and process certain series. In scientific analysis the data $y$ are traditionally the output of an 'experiment'. In setting up experiments or in performing econometric exercises to estimate model parameters, scientists choose which series are included in the observed data sample $y$. In designing analysis we want to ensure that the data used as observables are actually informative. In short, what data series should we choose to observe?

We focus on parameter inference with an infinite sample of data. An infinite supply of data - the complete population - makes it possible to perfectly characterise the probability distribution of the observed data. While clearly hypothetical, an infinite supply of data is the most favourable data context possible for estimation. If we cannot infer model parameters with an infinite sample then inference with finite samples can never be definitive about the parameters of interest. Speaking from a classical perspective, Christ (1966, p. 300) notes "there is little point [in] attempting to estimate structural parameters from a finite sample if even an infinite sample could not give the desired 
information about the parameters." We use this asymptotic perspective to inform a choice about which data series to use as observables.

One can think of our exercise as one in which the formulation of the model has preceded the collection of data. We then use the properties of the model to inform the collection of data. Proceeding 'as if' we know the population of data means there is no need to 'test' for identification, because there is no sampling variability. The approach is thus different to the classical weak-identification/weak-instrument literature. It is also distinct from the Bayesian approach suggested by Koop et al. (2013), which considers identification by examining how the dispersion of posterior distributions collapses in Monte Carlo experiments as simulated data samples are increased in size. Our approach can be thought of as highlighting the asymptotic properties of the model, and is unaffected by specific finite samples, samples that may not be fully representative of the population as a whole.

Although a somewhat contentious issue in earlier decades, ${ }^{4}$ Bayesian econometricians now seem to accept that identification presents as the same problem in both classical and Bayesian analysis, and depends on the properties of the likelihood of the data, see Poirier (1998) and Koop (2003, p. 291) for example. ${ }^{5}$ The likelihood principle of Bayesian analysis implies that the likelihood summarizes all the information in the data (Berger and Wolpert, 1988). It thus comes as no surprise that the likelihood takes centre stage when considering the mapping from data back to estimated parameters. Because the likelihood depends on the data series that are observed, it is clear that the choice of observables may, in principle, affect the identification of a model. We discuss why there is a choice of observables for DSGE models

\footnotetext{
${ }^{4}$ Hsiao (1983, p. 272) observed that it was 'unresolved' whether Bayesian theory required a different definition of identification to that used in classical analysis. Qin (1989) discusses the early history of identification analysis. Discussing likelihoods and identification in Bayesian analysis, Aldrich (2002) suggests that there was no agreement as to whether identification applied to priors, posteriors or likelihoods. A 2014 blog-post by Andrew Gelman, and associated comments, http://andrewgelman.com/2014/02/ 12/think-identifiability-bayesian-inference/, indicates that identification still remains a controversial topic in Bayesian statistical analysis.

${ }^{5}$ The emphasis on the likelihood for Bayesian identification dates back to at least Kadane (1975, p. 175) and Drèze (1975b, pp. 165, 167). Hsiao (1983), without attribution, associates this perspective with Savage. Aldrich (2002, p. 192) discusses the influence of Drèze in convincing Bayesians from Louvain that identification was a property of the likelihood.
} 
below.

The importance of the data for parameter estimates has been taken up for closed economy models, but this issue has not been pursued systematically for open economy models. The closed economy literature demonstrates that inferences can be sensitive to the data used to estimate models. GuerronQuintana (2010), for example, shows that the choice of observed data matters for parameter estimates, and thus impulse responses and out-of-sample forecasts. In particular, Guerron-Quintana finds that the parameter estimates of a Taylor rule and estimates of wage and price stickiness are materially affected by the choice of observables. Qu and Tkachenko (2012) raise similar concerns for the estimation of Taylor rule coefficients and Del Negro and Schorfheide (2008) show that 'standard macro time-series' may not enable one to discriminate between different degrees of wage and price rigidity. Iskrev (2010b) explores identification of the canonical Smets and Wouters (2007) model of a closed economy. Using the same data set as Smets and Wouters, Iskrev concludes that only 39 of the 41 parameters can be identified. In particular, identification issues arise because it is difficult to distinguish the Calvo price parameter in Smets and Wouters' model from the Kimball curvature parameter for goods aggregation; a similar problem arises with respect to the Calvo wage adjustment parameter and the Kimball curvature parameter for aggregating labour varieties. ${ }^{6}$ In section 2.4 we provide an illustration of a similar identification problem.

The choice of observables also affects variance decompositions. SchmittGrohé and Uribe (2012b) estimate a model with and without the relative price of investment and find that investment specific technology shocks play no role in generating economic fluctuations if the relative price of investment is included as an observable but a large role if this relative price is omitted. ${ }^{7}$

\footnotetext{
${ }^{6}$ See Smets and Wouters (2007) and Kimball (1995) for details of such aggregation.

${ }^{7}$ Greenwood et al. (2000), Fisher (2006), and Justiniano et al. (2010) all argue that investment-specific technology (IST) shocks are important drivers of cyclical fluctuations. Smets and Wouters (2007, fig. 1) report that investment shocks explain over twentypercent of the forecast error variance of GDP and the federal funds rate at some forecast horizons. Other estimates are even higher. A conventional IST shock is exactly equal to the inverse price of investment goods in terms of consumption. Kamber et al. (2015), see chapter 4 of this dissertation, show that IST shocks are not important drivers if a simple loan-to-value ratio friction is introduced into the model. Theory also matters for our interpretation of the underlying drivers of fluctuations.
} 
Similarly, Christiano et al. (2014, p. 29) find that "[o]ur conclusion that the risk shock is the most important shock depends crucially on including the four financial variables in our dataset." The dependence of these inferences on the data reflects the fact that variance decompositions are functions of the underlying structural parameters, and parameter estimates depend on the observed data. Arguably, the analysis in Guerron-Quintana (2010) and the other papers cited here raises questions about estimation rather than identification. The analysis shows that parameter estimates vary with particular samples of observables, but it is difficult to ascertain whether the results reflect something specific to the available data or whether they are fundamentally related to the choice of data series.

In addition to data and theory, the methodology used to estimate parameters also matters for identification. Canova and Sala (2009), for example, examine the use of impulse-response matching to identify parameters for a closed economy model similar in spirit to Smets and Wouters (2007). Canova and Sala find that impulse response matching is problematic for identification because impulses are nonlinear functions of the underlying structural parameters, making it difficult to invert the mapping back to the parameter space.

Identification may seem like an arcane econometric issue, but such issues are ultimately important for evaluations of welfare and hence also practical, normative choices about policy rules. Curvature parameters in preferences and production functions determine, for example, how egregious labour supply distortions are relative to distortions to the intratemporal or intertemporal composition of consumption. Ideally, the data will pin down these costs and we will not be forced to rely on purely subjective prior beliefs that may differ across evaluators, but it is important to understand when the data can - or cannot - perform this role.

In this paper we focus on identification in the context of a small open economy model. More specifically, we investigate whether foreign data are important for identification in our small open economy model. Exogenous or pre-determined variables are central to identify parameters in traditional systems of equations. Since small open economy models have additional exogenous (foreign) variables, there is the tantalising possibility that openness 
may facilitate parameter identification, even when identification is infeasible for a similar closed economy model. Are these foreign data series important in practice?

As noted earlier, identification issues in open economy models have not been investigated in any detail. While methodologically similar, small open economy (SOE) models can be both simpler and more complex than their closed economy counter-parts. For example, SOE models often abstract from the frictions that temper adjustment to shocks in closed economy models, such as capacity utilisation and possibly even capital, yet incorporate multiple sectors and multiple assets, such as domestic and foreign goods, and domestic and foreign bonds. We investigate whether the 'pathologies' that affect identification in medium-scale closed economy DSGE models also plague a generic model of a small open economy. We emphasize that this paper is about the use of foreign data for identification and estimation purposes, and is not at all concerned with the contribution of foreign variables for domestic cyclical fluctuations, which is another issue entirely.

To presage our results, we find that foreign data do not ease identification problems for the domestic parameters of the small open economy model that we use in our analysis. This result is of practical importance, as it implies that the domestic parameters of small open economy models can be estimated using only domestic data. The immateriality of foreign data for estimation is a practical boon, as the construction of 'rest-of-world' data is difficult for econometricians analysing open economies that have highly disaggregated trading patterns. We also illustrate a simple methodology that highlights some of the interdependencies that exist between structural parameters, interdependencies that are a feature of the underlying model and cannot be resolved through data alone.

The rest of the chapter proceeds as follows. In section 2.2 we discuss identification in the context of Bayesian estimation of a DSGE model. Section 2.3 briefly describes the small open economy that we use as our test-bed for identification analysis. In section 2.4 we explore identification in our small open economy dynamic stochastic general equilibrium model using the approach of Iskrev (2010b). Our primary objective is to understand identification issues in the context of this open economy model, focussing particularly on the 
implications of the data used to inform parameter estimation. We provide a contrast between identification in closed and open economy models to illustrate the role that exogenous foreign variables play for identification. Lastly, we conclude in section 2.5 .

\subsection{Identification of a Bayesian DSGE model}

In this section we provide the mathematical machinery used to describe a DSGE model, illustrate the nature of an identification problem in this context, discuss why a 'data choice' can arise in the estimation of DSGE models, and explain why we need to explore identification at multiple points in the parameter space. At its most basic level, we seek to ascertain whether it is possible to map uniquely from the 'data' back to the structural parameters that generated that data.

We begin by noting a conventional definition of model identification. ${ }^{8}$ Like Koopmans (1953), we conceive of a 'model' as a system of equations, and a family of probability distributions that govern the stochastic behaviour of any latent error terms. ${ }^{9}$ A model parameterised with a specific parameter vector $\theta$ will be referred to as an 'instantiation' of the model, following Chen (2016). ${ }^{10}$ A model is thus a set of instantiations.

Definition 1. Let $\mathcal{Y}$ be a sample space and $\mathcal{P}=\left\{P_{\theta}: \theta \in \Theta\right\}$ a family of probability distributions for $\mathcal{Y}$ indexed by the parameter vector $\theta$ belonging to the parameter space $\Theta$. This model is identifiable if for any $\theta_{1}, \theta_{2} \in \Theta$, $P_{\theta_{1}}(Y=y)=P_{\theta_{2}}(Y=y)$ for all $y \in \mathcal{Y}$ implies $\theta_{1}=\theta_{2}$.

The converse of this definition ${ }^{11}$ is that two distinct parameterisations $\left(\theta_{1} \neq \theta_{2}\right)$ can be distinguished from each other (identified) if there exists

\footnotetext{
${ }^{8}$ Bauwens et al. (1999, p. 41, definition 2.7) develop a definition of model identification from the identification of individual model parameters.

${ }^{9}$ See Florens et al. (2007, pp. 395-8) for a more elaborate description of a structural model.

${ }^{10}$ Christ (1966) uses the term 'structure' in place of instantiation, but this terminology is rather confusing in a modern context. An instantiation is common terminology in object-oriented programming.

${ }^{11}$ For variations on this theme see Koopmans and Reiers $\varnothing \mathrm{l}$ (1950, p. 169), Bowden (1973, definition 1), Kadane (1975, pp 176-7), Drèze (1975a, p. 161, definition 2.1), Pesaran (1987, ch. 6), Bauwens et al. (1999, p. 41, definition 2.7), Koop (2003, p. 291), Florens et al. (2007, p. 4), Rubio-Ramírez et al. (2010, p. 669), or Wechsler et al. (2013).
} 
some sample $y \in \mathcal{Y}$ such that the probabilities implied by the two parameterisations differ, $P_{\theta_{1}}(y) \neq P_{\theta_{2}}(y)$. As Koopmans and Reiers $\varnothing 1$ (1950) observe, the study of identification from this traditional simultaneous equations perspective relies on hypothetical, exact knowledge of the distribution of observables, as from a notionally infinite sample. ${ }^{12}$ Any discrepancy between two distribution functions will become evident as the sample asymptotes towards the population.

We apply this notion of identification to a dynamic stochastic general equilibrium model. Following the notation of Iskrev (2010b), a typical linearized DSGE model can be specified as:

$$
\Gamma_{0}(\theta) z_{t}=\Gamma_{1}(\theta) \underset{t}{\mathrm{E}} z_{t+1}+\Gamma_{2}(\theta) z_{t-1}+\Gamma_{3}(\theta) u_{t}
$$

where $z_{t}$ denotes an $n \times 1$ vector of (possibly unobserved) variables at time $t ; \mathrm{E}_{t}$ is a rational expectation formed at time $t$; and $u_{t}$ is an $m \times 1$ vector of independent and identically distributed Gaussian shocks with mean zero and an $m \times m$ covariance matrix corresponding to an identity matrix, $I_{m}$. $\Gamma_{0}(\theta), \Gamma_{1}(\theta)$ and $\Gamma_{2}(\theta)$ are $n \times n$ matrices of parameters, and $\Gamma_{3}(\theta)$ is an $n \times m$ matrix. The elements of these $\Gamma$ matrices are functions of the underlying structural parameters, denoted by $\theta$, which reflect the $k \times 1$ vector of parameters defining preferences and structural constraints.

If it exists, a unique reduced form solution can be expressed as: ${ }^{13}$

$$
z_{t}=A(\theta) z_{t-1}+B(\theta) u_{t}
$$

where $A(\theta)$ and $B(\theta)$ are parameter matrices that are functions of the structural parameters in $\theta$. Later we suppress the dependence on $\theta$ for the sake of brevity. When $u_{t}$ are $\operatorname{VAR}(1)$ errors then the solution will be a second order process in $z_{t}$, see Fukač and Pagan (2007).

Since some elements in $z_{t}$ may not be observed, it is common to cast the

\footnotetext{
${ }^{12}$ See also Manski (1993).

${ }^{13}$ For a general exposition of linear rational expectations models and their solutions see Binder and Pesaran (1995, 1997).
} 
model in state space form, appending an observation equation such as:

$$
y_{t}=s(\theta)+C z_{t}
$$

with $y_{t}$ denoting the observed variables with steady states $s(\theta)$. (We will assume that $z_{t}$ is demeaned relative to its own steady state.) The parameters $A, B$ and $s$ fully characterise the probability distribution of the variables in $y_{t}$. The matrix $C$ will typically be a deterministic selection matrix. In this paper we provide guidance as to what model variables should be selected by matrix $C$. The DSGE model specified in equations (2.1)-(2.3) is only identified if it is possible to uniquely map back from the parameters that define the distribution of observables, $A, B$ and $s$-or from functions of these parameters - to the structural parameters contained in $\theta$.

The mapping from structural parameters $\theta$ to reduced form parameters can be thought of as proceeding in two steps, from $\Theta \rightarrow \Gamma$ and thence from $\boldsymbol{\Gamma} \rightarrow \mathbf{A}$, using $\boldsymbol{\Gamma}$ to represent the space of admissible $\Gamma_{i}(i=0,1,2,3)$ matrices and defining $\mathbf{A}$ to be the space representing admissible reduced form parameters, i.e. $\left(\left((\operatorname{vec} s)^{\prime} \quad \operatorname{vec} A\right)^{\prime} \quad(\operatorname{vech} B)^{\prime} \quad(\operatorname{vec} C)^{\prime}\right)^{\prime} \in \mathbf{A}$. If each mapping, from the structural parameters to the parameters defined by the $\Gamma$ 's, and then from the $\Gamma$ 's to the reduced form parameters $\left(\begin{array}{llll}s & A & B & C\end{array}\right)$ is one-to-one and onto (bijective), then there will be a unique inverse mapping in return.

The second mapping, $\mathbf{\Gamma} \rightarrow \mathbf{A}$, is similar to that required for the simultaneous equations models (SEMs) common in the 1940s-1960s, though the equations of a DSGE model differ from a traditional SEM in four major respects:

i. The elements within DSGE $\Gamma$ 's are functions of the underlying structural parameters, i.e. there are many 'cross-equation restrictions'; ${ }^{14}$

ii. DSGE models typically contain expectations of some variables;

iii. DSGE models are dynamic and contain lagged regressors; and iv. Some variables are unobserved in DSGE models.

\footnotetext{
${ }^{14}$ Aldrich (1989, p. 15), quoting Haavelmo, argues that these equations are not therefore 'autonomous'.
} 
Let us make some observations about i), ii), iii) and iv) in turn.

With regards to i), the cross-equation restrictions implied by the DSGE model mean that individual elements in the $\Gamma$ matrices are determined by the smaller subset of underlying structural parameters, and consequently the elements of the $\Gamma$ 's are not strictly independent of each other. For example the $\Gamma$ elements may be (positively or negatively) correlated if the underlying structural parameters are perturbed. Consequently, some individual elements in the $\Gamma$ 's - and possibly entire equations - may not be necessary to identify the underlying structural parameters since the structural parameters may feature in multiple $\Gamma$ elements and hence be identifiable from other equations.

The inclusion of expectations, noted in ii), also distinguishes DSGE models from SEMs. Expectations can be treated as additional endogenous random variables, with variability that depends on the conditioning information set. In a traditional SEM the inclusion of an additional endogenous variable results in novel information, i.e. the additional population moments of the data series - which may or may not be helpful for identification. Incorporating rational expectations into a model is similarly ambiguous for the identification properties of the model. On one hand, rational expectations provides parameter restrictions that could assist identification, but on the other hand it may be difficult to disentangle coefficients on expectations from those associated with exogenous variables. The identification implications of expectations also relates to the lag specification of the model (issue iii). Pesaran (1987, ch. 6) and Koop et al. (2013, sn. 2) emphasize the importance of higher order dynamics in exogenous variables to achieve identification in linear rational expectations models and DSGE models respectively. These higher order dynamics serve to provide lagged instruments for the endogenous regressors in the model. ${ }^{15}$

The unobservability of some variables in DSGE models, issue iv) above,

\footnotetext{
${ }^{15}$ Sims (1980) argued that restrictions on lag lengths may be 'incredible', making them a weak foundation for identification, but his remark preceded the intensive use of Euler equations in RBC/DSGE models prompted by Kydland and Prescott (1982). Hatanaka (1975) explores identification of simultaneous equations models when there is uncertainty about lag lengths, and highlights the importance of exogenous variables whose lags are entirely excluded from the equation whose parameters we seek to identify. Here we assume that theory truly is informative about the lag lengths of endogenous and exogenous variables.
} 
is central to identification. In some cases 'observability' is dictated by circumstance since some variables may be intrinsically unobservable. Manski (1995, 2007), for example, considers identification when data are 'missing', as occurs when data are censored and so forth. Often the econometrician cannot control the censoring or 'missingness' of the data. In other cases, however, the econometrician may be able to choose which variables are members of the observed data set, $y{ }^{16}$

Identification analysis can be thought of as a component of experimental design (Kadane, 1975, p. 180). In Bayesian analysis an experiment is a triplet of data, parameters, and model, respectively $\left\{y, \theta_{j}, p\left(y \mid \theta_{j}, J\right)\right\}$, where $J$ is an index distinguishing a given model (Geweke, 2005, p. 98). By studying identification we learn, a priori, which data series we should observe and can develop theoretical insight into interdependencies between different parameters (Drèze, 1975a). What to observe, however, depends on the given the context of our theoretical restrictions. In the classic supply and demand example of Working (1927), identification is achieved by theoretical knowledge that there are exogenous or predetermined variables that perturb some equations and not others. For example, exogenous changes in supply help to trace out the demand curve with respect to price. Identification is provided by observing these exogenous or predetermined variables, in conjunction with a theory that says these variables enter the right-hand side of some equations and not others.

Observability is of especial interest in DSGE modelling. As Canova (2007, p. 440) and Canova et al. (2014) point out, the number of endogenous variables typically exceeds the number of shocks entering DSGE models. Consequently, as noted by Kim and Pagan (1995, p. 368) for example, the 'extra' observables may be written as deterministic functions of the others, implying that the variance-covariance matrix of errors is singular and the likelihood is hence undefined. Linear models with fewer shocks than observables must result in stochastic singularities, but Komunjer and Ng (2011, p. 2001) and Christiano (2012, fn. 9) note that singularities are the exception rather than

\footnotetext{
${ }^{16}$ Once a particular data sample $y=y_{0}$ is observed, the traditional Bayesian modes of analysis can be deployed: an experiment provides 'evidence' that enables the experimenter to make or update an inference regarding the parameter vector $\theta_{j}$, which in turn determines all other inferences that might be drawn from the model.
} 
the rule in the data, implying that there should be at least as many shocks as observable variables.

There are four methods commonly used to resolve stochastic singularities: ${ }^{17}$

i. Include additional structural shocks $;{ }^{18}$

ii. Include measurement errors; ${ }^{19}$

iii. Solve out variables from the optimality conditions until the number of shocks equals the number of variables; or

iv. Use a subset of observables less than or equal to the number of shocks entering the model. ${ }^{20}$

Chari et al. (2009) criticise the first strategy, suggesting that some of the newly-introduced shocks are 'dubiously structural'. Canova et al. (2014) caution that the first and second strategies "may distort parameter estimates and jeopardize inference", while Fernández-Villaverde et al. (2010) suggest that the introduction of measurement errors may complicate identification. Canova et al. (2014, p. 1099-1100) remark that the third strategy is problematic since "the convenient state-space structure of the decision rules is lost" and "the likelihood is an even more nonlinear function of the structural parameters and cannot necessarily be computed with standard Kalman filter recursions."

We focus on the fourth strategy, selecting a subset of variables as observables, which is arguably the most common. Ideally, one will select the subset of observables that is most informative for the estimation of the structural parameters. Komunjer and $\mathrm{Ng}$ (2011, p. 1997) comment on this difficulty, saying "[a]lthough we can drop some variables so that the system is full rank, the results will not be robust unless we know which variables are ancillary

\footnotetext{
${ }^{17}$ Bierens (2007) discusses a fifth method, which involves convoluting the model distribution with a non-singular distribution to match - by optimizing the structural parameters - an empirical model that has been convoluted similarly. See also Canova et al. (2014). Lai (2008) provides a variation on this theme.

${ }^{18}$ See for example Smets and Wouters (2007).

${ }^{19}$ As per Sargent (1989), Altug (1989) and Ireland (2004a).

${ }^{20}$ See Kim and Pagan (1995, p. 368).
} 
for the parameters of interest." See also Canova et al. (2014, p. 1100). Exactly which data series to retain, to estimate parameters in an open economy model, is the question we take up below. ${ }^{21}$

Now we turn to the methods used to determine model identification. Methodologically, identification is the study of simple conditions that imply model identifiability. Identification can be established in different ways because it is possible to distinguish 'the data' using different statistics. For example, conditions to ensure that parameterisations are distinct could be expressed in terms of the probability distributions, in terms of the data moments or moment generating functions, spectral properties, or even the cumulants (which can be obtained from the logarithm of the moment generating function). Modelling practitioners can then check that their models are identified by establishing that the required characteristics are present prior to estimation.

In a classic reference, Rothenberg (1971) demonstrates that, subject to a few technical conditions, a simultaneous equations model evaluated at a parameter vector $\theta_{0}$ will be locally identified provided that the information matrix evaluated at $\theta_{0}$ is non-singular, where the information matrix is the expectation of the matrix of second derivatives of the log-likelihood with respect to the parameter vector, with the expectation taken across all possible data. See also Bowden (1973). Iskrev (2010b) takes a different tack to Rothenberg and Bowden and uses the first and second order moments of observables implied by the model to establish identification. Mutschler (2015a) extends the conditions of Iskrev to higher order (nonlinear) approximations to the model, using the recursive framework outlined in Andreasen et al. (2018). Mutschler provides identification conditions for non-Gaussian models using the first four cumulants. For Gaussian models only the first two cumulants are non-zero and the higher order cumulants are therefore uninformative. Thus for Gaussian processes Mutschler's proposition ensuring local identification reverts to theorem 2 of Iskrev (2010b), which we replicate in appendix 2.A for convenience. Qu and Tkachenko (2012) specify conditions in the frequency domain and Komunjer and Ng (2011) specify identification

\footnotetext{
${ }^{21}$ Komunjer and $\mathrm{Ng}$ (2011) develop separate identification conditions for stochastically singular and non-singular models.
} 
conditions based on the parameter matrices of the state-space of the model. Canova et al. (2014) adopt the approach of Komunjer and $\mathrm{Ng}$ and apply it to the same data-choice question that we consider here. Canova et al. also apply the approach of Bierens (2007), computing an information measure that compares the conditional distribution of the singular theoretical model to the conditional distribution of the theoretical model; the singularity in the theoretical model is resolved by convoluting both distributions with a non-singular distribution. In an unpublished paper, Iskrev and Ritto (2016) apply a criterion based on the Fisher information matrix and cast doubt on the selection criteria of Canova et al.

The conditions applied to ensure identifiability are typically 'local' to a given parameter value $\theta_{0}$, as per definition 2 ; see also Iskrev (2010b, p. 192). A typical condition ensures that within some local neighbourhood of a candidate parameterisation there is no alternative parameterisation that has the same probabilistic implications for the data. Conditions that ensure global identifiability are more difficult to establish (Komunjer and Ng 2011, Iskrev 2010b, p. 192).

Definition 2. Let $\mathcal{Y}$ be a sample space and $\mathcal{P}=\left\{P_{\theta}: \theta \in \Theta\right\}$ a family of probability distributions for $\mathcal{Y}$ indexed by the parameter vector $\theta$ belonging to the parameter space $\Theta$. The parameter vector $\theta_{0} \in \Theta$ is locally identifiable if there exists an open neighbourhood $\mathrm{N}\left(\theta_{0}\right)$ around $\theta_{0}$ such that for any $\theta_{1} \in \mathrm{N}\left(\theta_{0}\right), P_{\theta_{0}}(Y=y)=P_{\theta_{1}}(Y=y)$ for all $y \in \mathcal{Y}$ implies $\theta_{0}=\theta_{1}$.

A number of the approaches discussed here are implementations of the inverse function theorem (see Rudin 1976, p. 221, replicated in appendix 2.B). To paraphrase, this theorem implies that a continuous vector-valued function will have a well-defined inverse, local to a particular point in its domain, if the matrix of partial derivatives evaluated at that point is invertible. Iskrev (2010b), for example, specifies a function mapping from the parameter space to the data moments and then evaluates the rank of the Jacobian matrix. Provided the Jacobian of this mapping has full rank, and is thus invertible, the inverse mapping from data moments to structural parameters will be bijective in a neighbourhood of the point being evaluated. Rothenberg's emphasis on the rank of the information matrix can be inter- 
preted in a similar light: the vector-valued function for Rothenberg (1971) is the score. As noted above, various authors have used the reduced form parameters, the spectrum, the Fisher information matrix, and cumulants, as the base function for identification.

At this point it is worth revisiting Lemma 1 of Kadane (1975, p. 177). This lemma and several definitions are reported in appendix 2.C for convenience. The lemma implies the following. If two functions are both identifying, if they can be used to distinguish points in the parameter space, then there must be a mapping from one function to the other. In principle, therefore, it does not matter which identifying function is used. Of course, as Canova and Sala (2009) show, it is possible to choose functions that are not identifying, so some caution is required. Which identifying function should then be chosen? In unpublished notes on identification, Canova suggests using the likelihood, since it contains all the information in the data. One might add that the Fisher information matrix, the negative of the expected value of the Hessian matrix of second derivatives of the log-likelihood, is a natural measure of the information contained in the data series.

Following Iskrev (2010b), we use the autocovariance function to characterise the data to explore the identification of a small open economy model across both data and parameter spaces. For a Gaussian vector autoregressive process the part of the score associated with the autoregressive coefficients (cf. the standard deviation parameters for the shocks) is a weighted average of the data variances and of the $p$ autocovariance matrices, where $p$ corresponds to the order of the vector autoregression. Thus, focussing on variances and autocorrelations provides an approximation to the score of a Gaussian likelihood. ${ }^{22}$ DSGE models are typically estimated with first order perturbation methods specified with Gaussian innovations, so that the machinery of the state-space can be used to compute the model likelihood, again supporting the case for the use of moments to identify the model.

We use a limited number of vector autocovariances to test for identification. In principle, the number of autocovariances might need to be large, but in practice we do not find this to be so. Given usual assumptions, the reduced form of the DSGE model is represented by a state-space model. In

\footnotetext{
${ }^{22}$ See appendix $2 . \mathrm{C}$ on page 90 for a brief note.
} 
turn, Hannan and Deistler (1988) note that state-space models have VARMA representations. As discussed by Kapetanios et al. (2007), a vector stochastic process $\left\{y_{t}: t \in \mathbb{Z}\right\}$ ( $\mathbb{Z}$ the set of integers) that follows a VAR will typically result in a VARMA process for some subset $y_{1 t}$ of the vector $y_{t}$, as would occur if some elements of $y_{t}$ are unobserved. Ravenna (2007) discusses conditions under which the reduced form of a DSGE model will have a finite order VAR. More generally, Ravenna's proposition 2.1 implies that the state-space model has a VARMA $(n+m, n+m-1)$ representation, where $m$ is the number of elements in the state vector and $n$ is the number of observables. Proposition 1 of Morris (2016) provides for a more parsimonious characterisation of the VARMA process, with fewer autoregressive and moving average terms. Nonetheless, autocovariance functions for VARMA processes are well defined. If our parameters uniquely change the low order autocovariances of our data then identification can still be achieved even though the reduced form has a more complicated lag structure.

It should be noted that identification is specific to a given model, and other competing theories and models could result in the same reduced form and hence be observationally equivalent. This observation is made both by Preston (1978) and Pesaran (1987). For example, one could baldly assert that the data generating process is a VARMA process with the same nonzero parameters as a structural model, and the data then cannot distinguish between the structural model and the alternative.

The data that are useful for identification may depend on the 'true' model, including its precise parameterisation, and the particular parameters of interest, which could be some sub-vector of the parameter vector $\theta$. Like most approaches in this literature, Iskrev's approach relies on properties local to a particular parameter vector, e.g. $\theta_{0}$. We use priors to guide the set of parameter values whose identification properties we explore. We think of the identification analysis that we are conducting here as a 'pre-posterior' analysis, just as one might simulate the model from priors to check that they do indeed result in plausible model properties before taking the model to the data. We evaluate the identification of the model across a carefully chosen set of points (vectors) drawn from the parameter space.

A complete identification analysis across the parameter space poses a 
substantial numerical challenge because of the curse of dimensionality. Suppose that the structural parameter vector has two elements and that each parameter is evaluated for identification at just five possible values. Then the number of parameter vectors that needs to be checked for identification is $5 \times 5=25$. Suppose instead that the structural parameter vector has ten elements, and suppose that each structural parameter is evaluated at eight different values. In this latter case the total number of distinct parameter vectors is $8^{10} \approx 1.07$ billion. Obviously, considering only eight distinct values for any single parameter is quite limited, since most DSGE priors for parameters are continuous rather than discrete.

Rather than focusing on a discrete lattice of points in the parameter space, we use Sobol' sequences to generate variates from the prior distributions. ${ }^{23}$ Sobol' sequences are a quasi-Monte Carlo method used to generate vector valued sequences whose domain is a unit hyper-cube. Quasi-random sequences produce faster rates of convergence to the integrals of interest than do conventional estimates derived from averaging 'actual' pseudo-random variates. Figure 2.1 illustrates bivariate Sobol' sequences (top pane) and pseudo random uniform variates (bottom pane), together with marginal distributions to the bottom and side. The bivariate plot clearly illustrates that the Sobol' sequences are not random, but the marginals and the plot of the distribution of points illustrate better coverage in this two dimensional hypercube, relative to pseudo random variates chosen 'at random'. The lack of randomness in the Sobol' sequences is immaterial for the analysis, as would also be the case for a lattice if it were practical.

Sobol' sequences can be used directly to sample uniformly from the finite support of random variables or can be used indirectly to sample from prior distributions, using inverse distribution functions where available. The goal with these sequences is to ensure that the proportion of points 'sampled' in a given subset of the (hyper) unit cube is as close to the volume of the subset as possible. One pitfall is that Sobol' sequences explore the edges of the hypercube, which is inconvenient when trying to map to a parameter space with an infinite support, such as the normal distribution for example. To avoid the extreme tails of the distribution, we truncate the exploration near

\footnotetext{
${ }^{23}$ Ratto (2008) uses Sobol' sequences in his sensitivity analysis.
} 
Figure 2.1: Two dimensional Sobol sequences and pseudo random uniform variates

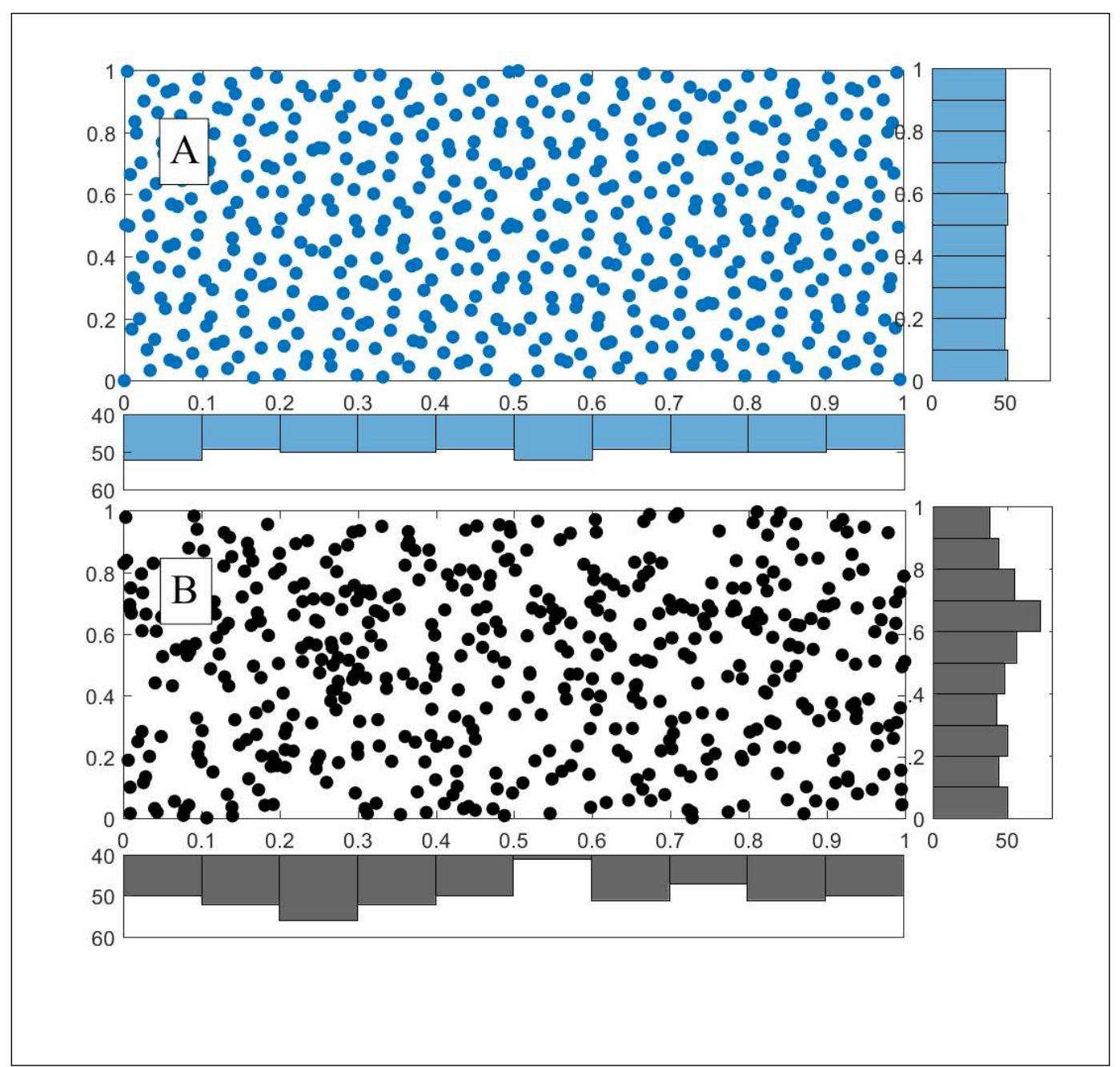

Panel A illustrates a bivariate (quasi-random) Sobol' sequence of 500 points. Panel B illustrates 500 pseudo random variates drawn from a bivariate uniform distribution. The histograms below and to the right of panels A and B illustrate the corresponding marginal distributions. The bins of the Sobol' sequence histograms exhibit much better coverage. Each of the ten bin should contain 50 values. 
the edges of the unit hyper-cube, at 0.001 and 0.999 . We sample from the prior distributions, to inform our pre-posterior efforts to determine which variables should be used as observables.

In the next section, we outline the model which forms the test-bed of our open economy analysis. We then turn to the results of our identification analysis in section 2.4 .

\subsection{Model}

In this section we briefly describe the log linearised equations of the open economy model used in our analysis. We use this model for illustrative purposes, and the approach described here could be applied to other DSGE models. As we use the model outlined in Justiniano and Preston (2010), we keep the description of the model fairly brief. The model has traditional New Keynesian elements of imperfect competition and sticky prices à la Calvo (1983). ${ }^{24}$ The linearized equations are presented in table 2.1 and 2.2; variable definitions are provided in table 2.3; and parameters are defined in tables 2.4 and 2.5.

As discussed by Justiniano and Preston, a variety of frictions are introduced from the closed economy literature, including exogenous habit in consumption, Calvo pricing for domestic goods, imports and wage-setting, and indexation of prices and wages. These features originated in papers such as Erceg et al. (2000), Fuhrer (2000), Woodford (2003), Smets and Wouters (2003, 2007), Christiano et al. (2005), and Monacelli (2005). We allow Calvo pricing for imports, which results in a 'law of one price gap' between the domestic currency price of imported goods and their foreign price (adjusting for the currency of denomination). Implicitly, we assume that it is possible to segment foreign and domestic markets, one of the three mechanisms considered by Benigno and Thoenissen (2003) to generate deviations from purchasing power parity. There are multiple distortions in the model, which are not perfectly correlated. Monetary policy, therefore, cannot simultane-

\footnotetext{
${ }^{24}$ Closely related predecessors in the open economy literature include Galí and Monacelli (2005) and Monacelli (2005). Kirsanova et al. (2006) is similar in spirit, but is a completemarkets version.
} 
ously eliminate all distortions, and policy-makers must trade off the various distortions when optimizing policy.

Foreign shocks affect domestic intertemporal substitution, via foreign bonds and trade in foreign and domestic goods. World prices affect the real exchange rate, which affects uncovered interest parity given incomplete financial markets ${ }^{25}$ foreign interest rates influence the domestic economy through the same channel. The general equilibrium character of the model means that foreign distortions, such as price stickiness for imports for example, affect the terms of trade and therefore have domestic welfare implications by affecting domestic consumption and labour supply, which are the arguments of the utility function of domestic agents and are thus the proximate drivers of welfare.

There is a limited array of financial assets in the economy. This open economy model is 'closed' by assuming a debt elastic interest rate premium (see Schmitt-Grohé and Uribe 2003), ensuring that transitory shocks do not have a permanent effect on the steady state of the model. For the purpose of this premium, debt is measured relative to the fraction of steady state consumption of the imported good. Foreign output (income) propagates through to demand for domestically-produced goods. The exchange rate and interest rates are the key prices that connect domestic and foreign resource allocations, and their macroeconomic dynamics. Taxes are assumed to match the subsidy needed to offset the markup stemming from imperfect competition, offsetting the steady-state distortion that would otherwise arise. Independent and identically distributed cost push shocks are introduced perturbing both domestic and import prices; these shocks can be thought of as exogenous variations in mark-ups, perhaps generated by stochastic changes in competitive pressures.

The equations governing foreign dynamics are akin to the equations for the domestic economy with a modest number of exceptions. First, consumption of domestically-produced imports by foreigners is negligible and is disregarded since the domestic economy is 'small'. Relatedly, there are no import cost push shocks for the foreign economy, and the terms of trade ef-

\footnotetext{
${ }^{25}$ Implicitly, complete risk-sharing is assumed within each country, but not between countries.
} 
fect on the foreign price level is negligible and hence dispensed with. Second, the law of one price is assumed to hold continuously in the foreign economy. Third, in the foreign monetary policy rule $\theta_{\Delta e^{*}}=0$, there is no response to the exchange rate. Fourth, foreigners are assumed to be net creditors and there is no interest elasticity with respect to the level of foreigners' debt. Note that $\eta^{*}$ is the foreign counterpart to $\eta$, the intratemporal substitution elasticity. ${ }^{26}$ We use $e_{t}$ to denote the nominal exchange rate and $s_{t}$ to denote the terms of trade, following the notation of Justiniano and Preston (2006) rather than the notation of Justiniano and Preston (2008) or (2010). ${ }^{27}$

Tables 2.1 and 2.2 report the linearised equations of the model for the domestic and foreign parts of the model, respectively. Definitions of the variables of the model can be found in table 2.3. Definitions of parameters can be found in 2.4 and 2.5. The prior distributions of the endogenous and exogenous parameters are reported in tables 2.6 and 2.7. We sample 10, 000 vectors from these prior distributions (using Sobol' sequences and inverse probability transforms) and then examine local identification at each vector given different sets of observables.

As we describe in equation (2.4), all exogenous shocks are stationary AR(1) processes, except domestic and foreign monetary policy shocks, which are independent and identically distributed (IID). The subscript · in the equation is replaced with $a, g, c H, c F, w, m, a^{*}, g^{*}, c^{*}, w^{*}, m^{*}$ as appropriate. $\check{\epsilon}_{\cdot t}$ is an IID innovation.

$$
\epsilon_{\cdot, t}=\rho . \epsilon_{\cdot, t-1}+\check{\epsilon}_{\cdot, t}
$$

\footnotetext{
${ }^{26}$ Justiniano and Preston denote the foreign intratemporal substitution elasticity using $\lambda$ in place of $\eta^{*}$.

${ }^{27}$ We have amended the notation for the linearised risk premium shock from $\phi_{t}$ to $\epsilon_{r p, t}$ for greater consistency with the notation of other exogenous shocks. We use $\theta$ 's with subscripts to denote parameters of monetary policy rules, with asterisks for foreign parameters and variables. The substitution elasticities for goods varieties have been changed from $\theta$ to $\epsilon$ and the substitution elasticity for labour varieties has been changed from $\theta_{w}$ to $\varepsilon_{w}$. $\theta$ with no subscript or a numerical subscript refers to the vector of parameters estimated using Bayesian methods. Exogenous technological progress is denoted $\epsilon_{a, t}$, ensuring greater notational consistency with the remaining exogenous variables.
} 
Table 2.1: Domestic linearised equations

1. Consumption Euler equation (with external habit)

$c_{t}-h c_{t-1}=E_{t}\left(c_{t+1}-h c_{t}\right)-\sigma(1-h)\left(r_{t}-E_{t} \pi_{t+1}\right)+\sigma(1-h)\left(\epsilon_{g, t}-E_{t} \epsilon_{g, t+1}\right)$

2. Goods market clearing

$y_{t}=(1-\tau) c_{t}+\left(\tau \eta^{*}+\tau \eta(1-\tau)\right) s_{t}+\tau \eta^{*} \psi_{F, t}+\tau y_{t}^{*}$

3. Law of one price (LOOP) gap

$\psi_{F, t} \equiv e_{t}+p_{t}^{*}-p_{F, t}$

4. Terms of trade

$\Delta s_{t}=\pi_{F, t}-\pi_{H, t}$

5. Real exchange rate ${ }^{\dagger}$

$q_{t}=e_{t}+p_{t}^{*}-p_{t}=\psi_{F, t}+(1-\tau) s_{t}$

6. Domestic Phillips curve

$\pi_{H, t}-\gamma_{H} \pi_{H, t-1}=\beta E_{t}\left(\pi_{H, t+1}-\gamma_{H} \pi_{H, t}\right)+\xi_{H}\left(w_{t}+\tau s_{t}-\epsilon_{a, t}\right)+\epsilon_{c H, t}$

7. Import Phillips curve

$\pi_{F, t}-\gamma_{F} \pi_{F, t-1}=\beta E_{t}\left(\pi_{F, t+1}-\gamma_{F} \pi_{F, t}\right)+\xi_{F} \psi_{F, t}+\epsilon_{c F, t}$

8. Wage Phillips curve

$\pi_{t}^{W}-\gamma_{W} \pi_{t-1}=\beta E_{t}\left(\pi_{t+1}^{W}-\gamma_{W} \pi_{t}\right)+\xi_{w}\left(v_{t}-w_{t}\right)$

9. Production function

$y_{t}=\epsilon_{a, t}+n_{t}$

10. Uncovered interest parity

$r_{t}-E_{t} \pi_{t+1}-\left(r_{t}^{*}-E_{t} \pi_{t+1}^{*}\right)=E_{t} \Delta q_{t+1}-\chi B_{t}-\epsilon_{r p, t}$

11. Flow budget constraint

$c_{t}+b_{t}=\beta^{-1} b_{t-1}-\tau\left(s_{t}+\psi_{F, t}\right)+y_{t}$

12. Monetary policy rule

$r_{t}=\theta_{r} r_{t-1}+\left(1-\theta_{r}\right)\left(\theta_{\pi} \pi_{t}+\theta_{y} y_{t}+\theta_{\Delta y} \Delta y_{t}+\theta_{\Delta e_{t}} \Delta e_{t}\right)+\epsilon_{m, t}$

13. Consumer price inflation

$\pi_{t}=\pi_{H, t}+\tau \Delta s_{t}$

Notes: ${ }^{\dagger}$ Note that $e_{t}$ can be substituted out of the model using the LOOP definition. There are three departures from Justiniano and Preston's priors: Indexation is introduced for imported goods; and both domestic and foreign cost push shocks here are $\mathrm{AR}(1)$ processes. 
Table 2.2: Foreign equations

25. Foreign Euler equation

$$
\begin{gathered}
y_{t}^{*}-h^{*} y_{t-1}^{*}=E_{t}\left(y_{t+1}^{*}-h^{*} y_{t}^{*}\right)-\left(1-h^{*}\right) \sigma^{*}\left(r_{t}^{*}-E_{t} \pi_{t+1}^{*}\right) \\
+\left(1-h^{*}\right) \sigma^{*}\left(E_{t} \epsilon_{g^{*}, t+1}-\epsilon_{g^{*}, t}\right)
\end{gathered}
$$

26. Foreign Phillips curve

$\pi_{t}^{*}-\gamma^{*} \pi_{t-1}^{*}=\beta^{*} E_{t}\left(\pi_{t+1}^{*}-\gamma^{*} \pi_{t}^{*}\right)+\xi^{*}\left(w_{t}^{*}-\epsilon_{a^{*}, t}\right)+\epsilon_{\pi^{*}, t}$

27. Foreign wage Phillips curve

$\pi_{w, t}^{*}-\gamma_{w^{*}} \pi_{w, t-1}^{*}=\beta^{*} E_{t}\left(\pi_{w^{*}, t+1}-\gamma_{w^{*}} \pi_{t}^{*}\right)+\xi_{w}^{*}\left(v_{t}^{*}-w_{t}^{*}\right)+\epsilon_{w^{*}, t}$

28. Foreign marginal rate of substitution

$v_{t}^{*}=\varphi^{*}\left(y_{t}^{*}-\epsilon_{a^{*}, t}\right)+\frac{1}{\left(\sigma^{*}\left(1-h^{*}\right)\right)}\left(y_{t}^{*}-h^{*} y_{t-1}^{*}\right)$

29. Foreign production

$y_{t}^{*}=\epsilon_{a^{*}, t}+n_{t}^{*}$

30. Foreign real wage

$w_{t}^{*}=w_{t-1}^{*}+\pi_{w, t}^{*}-\pi_{t}^{*}$

31. Foreign Monetary Policy

$r_{t}^{*}=\theta_{r}^{*} r_{t-1}^{*}+\left(1-\theta_{r}^{*}\right)\left(\theta_{\pi^{*}} \pi_{t+1}^{*}+\theta_{y^{*}, t} * y_{t}^{*}+\theta_{\Delta y^{*}}\left(y_{t}^{*}-y_{t-1}^{*}\right)\right)+\epsilon_{m^{*}, t}$

32. Change in exchange rate

$\Delta q_{t} \equiv q_{t}-q_{t-1}$

33. Change in terms of trade

$\Delta s_{t} \equiv s_{t}-s_{t-1}$

34. Foreign goods market clearing

$y_{t}^{*}=c_{t}^{*}$ 
Table 2.3: Variable definitions

Endogenous variables

\begin{tabular}{ll}
\hline$c_{t}$ & Consumption (real) \\
$b_{t}$ & Foreign bonds \\
$y_{t}$ & Output (real) \\
$r_{t}$ & Interest rate (nominal) \\
$q_{t}$ & Exchange rate (real) \\
$s_{t}$ & Terms of trade \\
$\pi_{t}$ & Headline inflation \\
$\pi_{H, t}$ & Home inflation \\
$\pi_{F, t}$ & Import inflation \\
$\pi_{W, t}$ & Wage inflation \\
$\psi_{F, t}$ & Deviation from law of one price \\
$v_{t}$ & Marginal rate of substitution \\
$p_{t}$ & Domestic price level \\
$p_{F, t}$ & Import price level \\
$e_{t}$ & Nominal exchange rate \\
$w_{t}$ & Wages $($ real) \\
$n_{t}$ & Labour supply \\
$\Delta y_{t}=$ & $y_{t}-y_{t-1} ; \Delta e_{t}=e_{t}-e_{t-1} ; \Delta s_{t}=s_{t}-s_{t-1}$
\end{tabular}

Foreign variables

$y_{t}^{*} \quad$ Foreign income (real)

$p_{t}^{*} \quad$ Foreign price level

$\pi_{t}^{*} \quad$ Foreign inflation

$\pi_{w, t}^{*} \quad$ Foreign wage inflation (nominal)

$r_{t}^{*} \quad$ Foreign interest rate (nominal)

$v_{t}^{*} \quad$ Foreign marginal rate of substitution

$w_{t}^{*} \quad$ Foreign wages (real)

Exogenous shocks

$\epsilon_{a, t} \quad$ Technology shock

$\epsilon_{g, t} \quad$ Demand shock

$\epsilon_{w, t} \quad$ Labour supply shock

$\epsilon_{c H, t} \quad$ Domestic cost push shock

$\epsilon_{c F, t} \quad$ Import cost push shock

$\epsilon_{r p, t} \quad$ Risk premium shock

$\epsilon_{m, t} \quad$ Monetary policy shock

$\epsilon_{a^{*}, t} \quad$ Foreign technology shock

$\epsilon_{g^{*}, t} \quad$ Foreign demand shock

$\epsilon_{w^{*}, t} \quad$ Foreign labour supply shock

$\epsilon_{c^{*}, t} \quad$ Foreign cost push shock

$\epsilon_{m^{*}, t}$ Foreign monetary policy shock 
Table 2.4: Domestic parameter definitions

Preference \& structural parameters

$\begin{array}{ll}\beta & \text { Discount factor } \\ \varepsilon & \text { Substitution elasticity between goods varieties } \\ \varepsilon_{w} & \text { Substitution elasticity between labour varieties } \\ \varphi & \text { Inverse Frisch (labour supply) elasticity } \\ \sigma & \text { Intertemporal substitution elasticity } \\ \alpha_{H} & \text { Calvo domestic prices } \\ \alpha_{F} & \text { Calvo import prices } \\ \alpha_{W} & \text { Calvo wages } \\ \gamma_{H} & \text { Indexation domestic prices } \\ \gamma_{W} & \text { Indexation wages } \\ h & \text { Consumption habit } \\ \tau & \text { Degree of openness (inverse of home bias) } \\ \eta & \text { Domestic intratemporal subst. elasticity home/foreign goods } \\ \chi & \text { Interest elasticity w.r.t. debt }\end{array}$

Domestic policy parameters

$\theta_{\pi} \quad$ Response to inflation

$\theta_{y} \quad$ Response to output gap

$\theta_{\Delta y} \quad$ Response to output growth

$\theta_{\Delta e}$ Response to exchange rate change

$\theta_{r} \quad$ Interest rate smoothing

Auxiliary parameter definitions

$$
\begin{aligned}
& \xi_{H} \equiv\left(1-\alpha_{H}\right)\left(1-\alpha_{H} \beta\right) / \alpha_{H} \\
& \xi_{F} \equiv\left(1-\alpha_{F}\right)\left(1-\alpha_{F} \beta\right) / \alpha_{F} \\
& \xi_{w} \equiv\left(1-\alpha_{W}\right)\left(1-\alpha_{W} \beta\right) /\left(\alpha_{W}\left(1+\varphi \varepsilon_{w}\right)\right) \\
& \xi^{*} \equiv\left(1-\alpha^{*}\right)\left(1-\beta^{*} \alpha^{*}\right) / \alpha^{*} \\
& \xi_{w}^{*} \equiv\left(1-\alpha_{W^{*}}\right)\left(1-\alpha_{W^{*}} \beta^{*}\right) /\left(\alpha_{W^{*}}\left(1+\varphi^{*} \varepsilon_{w}^{*}\right)\right)
\end{aligned}
$$

Exogenous shock persistences \& standard deviations

$\begin{array}{ll}\rho_{a} & \operatorname{AR}(1) \text { persistence technology shock } \\ \rho_{g} & \operatorname{AR}(1) \text { persistence exogenous demand shock } \\ \rho_{c H} & \operatorname{AR}(1) \text { persistence domestic cost push shock } \\ \rho_{c F} & \operatorname{AR}(1) \text { persistence import cost push shock } \\ \rho_{w} & \operatorname{AR}(1) \text { persistence labour supply shock } \\ \rho_{r p} & \operatorname{AR}(1) \text { persistence risk premium shock } \\ \sigma_{a} & \text { Standard deviation technology shock } \\ \sigma_{g} & \text { Standard deviation consumption preference shock } \\ \sigma_{c H} & \text { Standard deviation domestic cost push shock } \\ \sigma_{c F} & \text { Standard deviation import cost push shock } \\ \sigma_{w} & \text { Standard deviation labour supply shock } \\ \sigma_{r p} & \text { Standard deviation risk premium shock } \\ \sigma_{m} & \text { Standard deviation monetary policy shock }\end{array}$


Table 2.5: Foreign parameter definitions

Preference \& structural parameters

$\beta^{*} \quad$ Foreign discount factor

$\varepsilon^{*} \quad$ Foreign substitution elasticity between varieties

$\varepsilon_{w}^{*} \quad$ Foreign substitution elasticity between labour varieties

$\varphi^{*} \quad$ Foreign inverse Frisch (labour supply) elasticity

$\sigma^{*} \quad$ Foreign intertemporal substitution elasticity

$\alpha_{H^{*}} \quad$ Foreign Calvo domestic prices

$\alpha_{W^{*}} \quad$ Foreign Calvo import prices

$\gamma_{H^{*}} \quad$ Foreign indexation domestic prices

$\gamma_{w^{*}} \quad$ Foreign indexation wages

$h^{*} \quad$ Foreign consumption habit

$\eta^{*} \quad$ Foreign price elasticity of foreign demand for domestic goods

Foreign policy parameters

$\theta_{\pi^{*}} \quad$ Foreign response to inflation

$\theta_{y^{*}} \quad$ Foreign response to output gap

$\theta_{\Delta y^{*}} \quad$ Foreign response to output growth

$\theta_{r^{*}} \quad$ Foreign interest rate smoothing

Foreign exogenous shock persistences \& standard deviations

$\rho_{a^{*}} \quad \mathrm{AR}(1)$ persistence foreign technology shock

$\rho_{g^{*}} \quad \mathrm{AR}(1)$ persistence foreign consumption preference shock

$\rho_{l^{*}} \quad \operatorname{AR}(1)$ persistence foreign labour supply shock

$\rho_{\pi^{*}} \quad \operatorname{AR}(1)$ persistence foreign cost push shock

$\sigma_{a^{*}} \quad$ Standard deviation foreign technology shock

$\sigma_{g^{*}} \quad$ Standard deviation foreign consumption preference shock

$\sigma_{w^{*}} \quad$ Standard deviation foreign labour supply shock

$\sigma_{\pi^{*}} \quad$ Standard deviation foreign cosh push shock

$\sigma_{m^{*}} \quad$ Standard deviation foreign monetary policy shock 
Table 2.6: Prior distributions domestic parameters

Symbol Description Prior Mean Std-Dev.

Preference \& structural parameters

\begin{tabular}{lllll}
\hline$\beta$ & Discount factor & $C$ & 0.99 & - \\
$\varepsilon$ & Substitution elasticity between goods varieties & $C$ & 8.00 & - \\
$\varepsilon_{w}$ & Substitution elasticity between labour varieties & $C$ & 8.00 & - \\
$\chi$ & Interest elasticity to debt & $C$ & 0.01 & - \\
$\varphi$ & Inverse Frisch (labour supply) elasticity & $N$ & 1.00 & 0.30 \\
$\sigma$ & Intertemporal substitution elasticity & $N$ & 1.00 & 0.40 \\
$\alpha_{H}$ & Calvo domestic prices & $\beta$ & 0.60 & 0.10 \\
$\alpha_{F}$ & Calvo import prices & $\beta$ & 0.50 & 0.20 \\
$\alpha_{W}$ & Calvo wages & $\beta$ & 0.60 & 0.10 \\
$\gamma_{H}$ & Indexation domestic prices & $\beta$ & 0.50 & 0.20 \\
$\gamma_{W}$ & Indexation wages & $\beta$ & 0.50 & 0.20 \\
$h$ & Consumption habit & $\beta$ & 0.50 & 0.10 \\
$(1-\tau)$ & Degree of home bias & $\beta$ & 0.71 & 0.02 \\
$\eta$ & Intratemporal subst. elasticity home/foreign goods & $N$ & 0.90 & 0.10 \\
$\theta_{\pi}$ & Response to inflation & $N$ & 1.80 & 0.30 \\
$\theta_{y}$ & Response to output gap & $G$ & 0.25 & 0.13 \\
$\theta_{\Delta y}$ & Response to output growth & $N$ & 0.30 & 0.20 \\
$\theta_{\Delta e}$ & Response to exchange rate change & $G$ & 0.30 & 0.20 \\
$\theta_{r}$ & Interest rate smoothing & $\beta$ & 0.60 & 0.20
\end{tabular}

Foreign structural parameters

\begin{tabular}{lllll}
\hline$\beta^{*}$ & Discount factor & $C$ & 0.99 & - \\
$\varepsilon^{*}$ & Substitution elasticity between goods varieties & $C$ & 8.00 & - \\
$\varepsilon_{w}^{*}$ & Substitution elasticity between labour varieties & $C$ & 8.00 & - \\
$\varphi^{*}$ & Inverse Frisch (labour supply) elasticity & $N$ & 1 & 0.3 \\
$\sigma^{*}$ & Intertemporal substitution elasticity & $N$ & 1 & 0.3 \\
$\alpha_{H}^{*}$ & Calvo prices & $\beta$ & 0.6 & 0.1 \\
$\alpha_{W}^{*}$ & Calvo wages & $\beta$ & 0.6 & 0.1 \\
$\gamma_{H}^{*}$ & Indexation prices & $\beta$ & 0.5 & 0.15 \\
$\gamma_{W}^{*}$ & Indexation wages & $\beta$ & 0.5 & 0.15 \\
$h^{*}$ & Consumption habit & $\beta$ & 0.50 & 0.1 \\
$\eta^{*}$ & Intratemporal subst. elasticity home/foreign goods & $N$ & 1.5 & 0.50 \\
$\theta_{\pi}^{*}$ & Response to inflation & $N$ & 1.80 & 0.30 \\
$\theta_{y}^{*}$ & Response to output gap & $G$ & 0.25 & 0.13 \\
$\theta_{\Delta y}^{*}$ & Response to output growth & $N$ & 0.30 & 0.20 \\
$\theta_{r}^{*}$ & Interest rate smoothing & $\beta$ & 0.60 & 0.20 \\
\hline
\end{tabular}

Note: Prior distributions: $C=$ Calibrated; $\beta=$ Beta; $N=$ Normal; $G=$ Gamma; $G^{-1}=$ Inverse Gamma. For symmetry with the domestic economy we have included $\theta_{\Delta y}^{*}$ in the rest-of-world policy rule. Prior parameter values largely follow Justiniano and Preston (2010, p. 67). 
Table 2.7: Prior distributions exogenous parameters Exogenous shock persistences \& standard deviations

Symbol Description

$\begin{array}{lllll}\rho_{a} & \mathrm{AR}(1) \text { persistence technology } & \beta & 0.60 & 0.20\end{array}$

$\begin{array}{lllll}\rho_{g} & \mathrm{AR}(1) \text { persistence exogenous demand } & \beta & 0.60 & 0.20\end{array}$

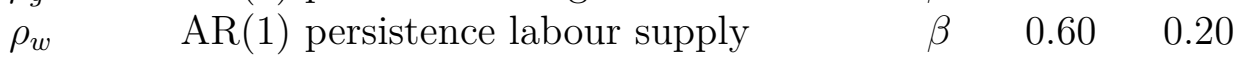

$\begin{array}{lllll}\rho_{c H} & \mathrm{AR}(1) \text { persistence domestic cost push } & \beta & 0.60 & 0.20\end{array}$

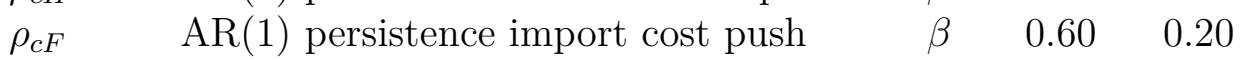

$\begin{array}{lllll}\rho_{r p} & \mathrm{AR}(1) \text { persistence risk premium } & \beta & 0.60 & 0.20\end{array}$

$\rho_{m} \quad$ No persistence in monetary $\quad$ C $\quad 0 \quad-$

$\begin{array}{lllll}\rho_{a}^{*} & \mathrm{AR}(1) \text { persistence foreign technology } & \beta & 0.80 & 0.15\end{array}$

$\begin{array}{lllll}\rho_{g}^{*} & \mathrm{AR}(1) \text { persistence foreign preference } & \beta & 0.80 & 0.15\end{array}$

$\begin{array}{lllll}\rho_{w}^{*} & \mathrm{AR}(1) \text { persistence labour supply } & \beta & 0.80 & 0.15\end{array}$

$\begin{array}{lllll}\rho_{c H}^{*} & \mathrm{AR}(1) \text { persistence in foreign cost-push } & \beta & 0.8 & 0.15\end{array}$

$\rho_{m}^{*} \quad$ No persistence in foreign monetary $\quad$ C 0 -

Shock standard deviations

\begin{tabular}{lllll}
\hline$\sigma_{a}$ & Technology & $G^{-1}$ & 0.50 & 1.00 \\
$\sigma_{g}$ & Consumption preference & $G^{-1}$ & 1.00 & 1.00 \\
$\sigma_{w}$ & Labour supply & $G^{-1}$ & 2.00 & 1.00 \\
$\sigma_{c H}$ & Domestic cost push & $G^{-1}$ & 0.15 & 1.00 \\
$\sigma_{c F}$ & Import cost push & $G^{-1}$ & 1.00 & 1.00 \\
$\sigma_{r p}$ & Risk premium & $G^{-1}$ & 1.00 & 1.00 \\
$\sigma_{m}$ & Monetary policy & $G^{-1}$ & 0.15 & 1.00 \\
$\sigma_{a}^{*}$ & Technology & $G^{-1}$ & 1.00 & 2.00 \\
$\sigma_{g}^{*}$ & Foreign consumption preference & $G^{-1}$ & 2.00 & 2.00 \\
$\sigma_{w}^{*}$ & Labour supply & $G^{-1}$ & 4.00 & 2.00 \\
$\sigma_{c H}^{*}$ & Foreign cost push & $G^{-1}$ & 0.25 & 2.00 \\
$\sigma_{m}^{*}$ & Foreign monetary & $G^{-1}$ & 0.25 & 2.00 \\
\hline
\end{tabular}

Note: Prior distributions: $C=$ Calibrated; $\beta=$ Beta; $N=$ Normal; $G=$ Gamma; $G^{-1}=$ Inverse Gamma The prior parameter values follow Justiniano and Preston (2010, p. 67). 


\subsection{Results}

\subsubsection{Identification of foreign parameters}

Before beginning our open economy analysis, we first consider the identification of a closed economy counterpart to this small open economy model. This exercise allows us to ascertain the features of the model - independent of openness - that are subject to identification problems. Conveniently, the foreign block of the model represents just such a counterpart. We strip off the domestic economy and examine how the choice of foreign (i.e. closed economy) data series influence identification of the foreign structural parameters.

\section{Mapping from $\Theta$ to $\Gamma$}

The linearised equations in section 2.3 above are a specific model corresponding to the expectational equations (2.1). As noted in section 2.2, we can think of the mapping from structural parameters to the reduced form as occurring in two steps - from $\theta$ 's to $\Gamma$ 's and then from $\Gamma$ 's to $s, A, B, C$, and thence to the moments of the data, whose space we will denote $\mathbf{M}$. Define the first mapping to be $f: \Theta \rightarrow \Gamma$ and the second mapping $g: \boldsymbol{\Gamma} \rightarrow \mathbf{M}$. We assume that $\boldsymbol{\Gamma}$ is the range of $f$ and $\mathbf{M}$ is the range of $g$, so that each space is onto with respect to the two functions. Like the bulk of the DSGE literature, we assume that the model has a unique, determinate equilibrium. ${ }^{28}$ Thus, the admissible space $\boldsymbol{\Gamma}$ is restricted to ensure that the mapping $g(\Gamma)$ for $\Gamma \in \boldsymbol{\Gamma}$ is single-valued. The space $\boldsymbol{\Gamma}$ serves as a bridge between $\Theta$ and $\mathbf{M}$ : all roads pass through this bridge. ${ }^{29}$

A function that is one-to-one and onto has a well-defined inverse. We make use of this observation to focus attention on these two mappings separately. Theorem 1 illustrates the importance of the mapping $f: \Theta \rightarrow \Gamma$.

\footnotetext{
${ }^{28}$ There are exceptions to this generalisation. See, for example, Lubik and Schorfheide (2004a,b), Farmer et al. (2015) and Tchatoka et al. (2017).

${ }^{29}$ For those fond of Nordic noir television or massive engineering projects, it might be helpful to think of $\boldsymbol{\Gamma}$ as the Peberholm island in the middle of (the) Øresund (Sound), between Copenhagen in Denmark and Malmö in Sweden. The functions $f$ and $g$ can be thought of as the Øresund bridge and the Drogden tunnel that map to the island.
} 
Theorem 1. Let $f: \boldsymbol{\Theta} \rightarrow \boldsymbol{\Gamma}$ and $g: \boldsymbol{\Gamma} \rightarrow \mathbf{M}$ be functions. If $g \circ f(\theta)$ is one-to-one then $f$ must be one-to-one.

For a simple proof, see appendix 2.E.

We argue here that it is useful to consider each mapping $f$ and $g$ in turn. Traditional identification techniques define the properties of the model using its reduced form, by its parameters, spectrum, or autocovariances. But it is also true that the properties of the model are defined by the expectational equations. Fortunately, the same techniques to locally identify a bijection can be considered in each case. To examine the vector mapping $f: \Theta \rightarrow \boldsymbol{\Gamma}$, we estimate the Jacobian matrix of $f$, which is the matrix of partial derivatives of all functions in $f$ with respect to each parameter $\theta_{i}$, where subscript $i$ indexes the $i^{\text {th }}$ element of $\theta$. The rank of this Jacobian matrix specifies the number of elements of $\theta$ that can be uniquely identified. Given that the function $f$ is explicitly defined, the Jacobian can be computed symbolically.

We take each of the parameters in the expectational equations, excluding deterministic coefficients, ${ }^{30}$ and collate them into a vector. We then compute the matrix of partial derivatives with respect to the elements in the vector $\theta$. Consider the case where each structural parameter maps uniquely to just a single element in the $\Gamma$ 's. Then the Jacobian is simply an identity matrix. A structural parameter $\theta_{i}$ that maps uniquely to just a single element $\Gamma_{j}$ poses no identification difficulty, and so we can simplify the multivariate mapping, eliminating both the parameter $\theta_{i}$ and the associated equation. In our model, we eliminate the standard errors of the variance terms and the $\operatorname{AR}(1)$ coefficients of the structural errors. We also eliminate duplicate 'equations'; when an element $\Gamma_{i}$ is a scalar multiple of $\Gamma_{j}$, for $i \neq j$, we eliminate one of these equations.

\footnotetext{
${ }^{30} \mathrm{We}$ exclude these deterministic parameters because the derivative vector associated with them would consist solely of zeros.
} 
We are left with the following system of equations: ${ }^{31}$

$$
f=\left(\begin{array}{c}
h^{*} \\
\left(1-h^{*}\right) \sigma^{*} \\
\beta^{*} /\left(1+\beta^{*} \gamma^{*}\right) \\
\gamma^{*} /\left(1+\beta^{*} \gamma^{*}\right) \\
\left(1-\beta^{*} \alpha^{*}\right)\left(1-\alpha^{*}\right) /\left(\alpha^{*}\left(1+\beta^{*} \gamma^{*}\right)\right) \\
\gamma_{w}^{*} \\
\beta^{*} \\
-\beta^{*}\left(\gamma_{w}^{*}\right) \\
\left(1-\beta^{*} \alpha_{w}^{*}\right)\left(1-\alpha_{w}^{*}\right) /\left(\alpha_{w}^{*}\left(1+\left(1+\varepsilon_{w}^{*}\right) / \varepsilon_{w}^{*} \varphi^{*}\right)\right) \\
\varphi_{*} \\
1 /\left(\sigma_{*}\left(1-h^{*}\right)\right) \\
-1 /\left(\sigma_{*}\left(1-h^{*}\right)\right) S h^{*} \\
\theta_{r}^{*} \\
\left(1-\theta_{r}^{*}\right) \theta_{\pi}^{*} \\
\left(1-\theta_{r}^{*}\right) \theta_{y}^{*} \\
\left(1-\theta_{r}^{*}\right) \theta_{d y}^{*}
\end{array}\right)
$$

and we take derivatives with respect to the following vector of foreign structural parameters: $\beta^{*}, \epsilon_{W}^{*}, \varphi_{*}, \sigma^{*}, \alpha^{*}, \alpha_{w}^{*}, \gamma^{*}, \gamma_{w}^{*}, h^{*}, \eta^{*}, \theta_{\pi}^{*}, \theta_{y}^{*}, \theta_{d y}^{*}, \theta_{r}^{*}$. For the sake of brevity we omit reporting this Jacobian matrix.

By computing a basis for the null-space ${ }^{32}$ of the Jacobian matrix of $f$, we can ascertain which, if any, parameters are either not locally identified or are under-identified. ${ }^{33}$ As Iskrev (2010a) notes, a lack of identification arises in two ways: i) a parameter may not feature in the likelihood; and/or ii) two or more parameters may not be separately identifiable. The first possibility presents itself as a column of zeroes in the Jacobian and can be ascertained directly. If the second alternative arises then two or more columns of the Jacobian are linearly dependent. In this second case we determine which

\footnotetext{
${ }^{31}$ See the parameters in table 2.2 .

${ }^{32}$ Remember that the null space of a matrix $A$ is the set of vectors x such that $A x=0$. If $A$ has full rank then the only vector in the null space is the vector $\mathbf{0}$.

${ }^{33}$ Bonaldi (2010) also makes use of ranks to determine how many parameters can be identified, and the null spaces of these Jacobian matrices to determine which parameters are dependent and therefore unidentified.
} 
columns are interdependent by computing the null space of the Jacobian matrix. The non-zero elements of each vector in the basis of the null space determines a combination of columns of $f^{\prime}(\theta)$ that maps to the null vector, thus illustrating which columns are dependent.

To understand local identification problems in this context note that for any arbitrary $\theta_{0}$ in the domain of the parameter space we can specify the following first order approximation:

$$
f\left(\theta_{0}+\delta\right) \approx f\left(\theta_{0}\right)+f^{\prime}\left(\theta_{0}\right) \delta
$$

But if $\delta$ is in the null space of $f^{\prime}(\theta)$, then the second term on the right hand side equals zero. Thus, by moving certain parameters by carefully calibrated amounts we can generate the same $\Gamma$ parameters, implying that both $\theta_{0}$ and $\theta_{0}+\delta$ could have generated the same expectational equations and hence the same data. If the parameter vector is locally identified at $\theta_{0}$ then the only element in the null space of $f^{\prime}\left(\theta_{0}\right)$ will be the null vector, $\delta=\mathbf{0}$.

The system of equations $f$ specified here can be expressed symbolically, and both the Jacobian and null space can be computed symbolically, using the jacobian and null commands from the Symbolic Math Toolbox. For the particular case here, Matlab's Symbolic toolbox indicates that the following 
two vectors form a basis for the null space of $f^{\prime}(\theta)$.

$$
\left(\begin{array}{cc}
0 & 0 \\
\frac{-\left(\epsilon_{W}^{*}\left(\beta^{*}\left(\alpha_{w}^{*}\right)^{2}-1\right)\left(\epsilon_{W}^{*}+\varphi^{*}+\epsilon_{W}^{*} \varphi^{*}\right)\right)}{\left(\alpha_{w}^{*} \varphi^{*}\left(\alpha_{w}^{*} \beta^{*}-1\right)\left(\alpha_{w}^{*}-1\right)\right)} & 0 \\
0 & 0 \\
0 & 0 \\
0 & 0 \\
1 & 0 \\
0 & 0 \\
0 & 0 \\
0 & 0 \\
0 & 1 \\
0 & 0 \\
0 & 0 \\
0 & 0 \\
0 & 0
\end{array}\right)
$$

The first column indicates that the second and sixth parameters, $\varepsilon_{w}^{*}$ and $\alpha_{w}^{*}$ are not separately identifiable. The effect of changing $\varepsilon_{w}^{*}$ on the $\Gamma$ parameters can be perfectly offset by a suitably-sized amendment to the parameter $\alpha_{w}^{*}$. Conversely, the second column of the basis illustrates that the tenth parameter $\eta^{*}$ has no effect on any of the $\Gamma^{\prime}$ 's and hence cannot be identified at all. (This is a quirk of the model; the relative price affects foreign demand for domestic goods, but the domestic economy is negligible in size for total demand from the foreign economy's perspective. Thus, $\eta^{*}$ only features in the domestic equations.)

Looking directly at the expectational equations in table 2.2 , we see that the Phillips curve equations for foreign inflation and foreign wage inflation have parameters $\xi^{*}$ and $\xi_{w}^{*}$ that are functions of Calvo parameters, discount rates, and in the case of wages the foreign Frisch elasticity. The discount factor $\beta^{*}$ enters both the wage and price Phillips curve. In simple models $\beta^{*}$ is traditionally tied down by the steady-state interest rate, calibrated $\approx 0.99$. Given a value for $\beta^{*}$ the $\xi^{*}$ parameter is a monotonic function of the structural Calvo parameter $\alpha^{*}$, and therefore the Calvo parameter can be 
recovered if this $\xi^{*}$ parameter is identifiable. The wage Phillips curve is more complex. The parameters $\varepsilon_{w}^{*}$ and $\alpha_{w}^{*}$ only enter $\xi_{w}^{*}$ and these parameters are only separately identifiable because $\varepsilon_{w}^{*}$ is calibrated.

With a multiplicity of equations, it can be difficult to track down which structural parameters are subject to such identification problems. The approach here provides a straightforward and easily implementable method to determine which parameters are subject to these types of identification problems. As models grow in complexity, evaluations of this kind will be particularly valuable in providing insight into the interdependencies that exist between new, unfamiliar parameters and the more-familiar parameters that govern simpler models.

\section{Identification of $\Gamma$}

We now turn to the second mapping, from the expectational equations to the reduced form. Instead of explicitly identifying the underlying structural parameters, $\theta$, we consider whether it is possible to map from the data moments back to the $\Gamma$ matrices, ignoring the cross-equation restrictions that link different elements in $\Gamma$ together. Implicitly, the priors for the elements in $\theta$ define priors for the elements in the $\Gamma$ matrices. We have 18 non-deterministic elements in the $\Gamma$ matrices, which we denote $\Gamma_{i}$ for $i=1, \ldots, 18$. We use these priors to consider local identification at different points in the space $\boldsymbol{\Gamma}$, together with 5 parameters for the standard deviations and 5 persistence parameters from the autoregressive shock terms, 28 parameters in all.

For each set of observables (which defines a set of moments), and for each parameter vector drawn from our sample of 10,000, we evaluate the ranks of the Jacobians of the moments with respect to parameters to see whether different observables affect the identification of the model. In principle, different parameter vectors may have different 'local' identification characteristics even for the same set of observables, but as we detail below, the analysis indicates the identification patterns in our model are largely unrelated to the exact parameterisation of the model. In other words, changing the parameterisation of the model does not change the rank of the Jacobian matrix.

We draw observables from the following set: foreign output $y^{*}$; foreign inflation $\pi^{*}$; foreign wage inflation $\pi_{W}^{*}$; the foreign interest rate $r^{*}$; foreign 
wages $w^{*}$; and hours worked $n^{*}$, and examine how identification is affected by the choice of observables. There are five foreign/closed-economy shocks (technology, 'demand', wage and price markup, and monetary policy shocks), thus to avoid stochastic singularity we can have no more than five observables.

When we use all six observables, we find that there is only one identification issue that arises when ignoring the cross-equation restrictions. Namely the $\Gamma_{5}$ attached to $E_{t} \epsilon_{g^{*}, t+1}-\epsilon_{g^{*}, t}$ is not separately identifiable from the standard deviation of the aggregate demand shock $\sigma_{g^{*}}{ }^{34}$ This identification problem is not problematic for the estimation of $\theta$ because the cross-equation restrictions imply that $\Gamma_{4}=\Gamma_{5}$.

Table 2.8 illustrates that, across the 10,000 parameter draws, at most 27 of $28 \Gamma$ parameters can be identified when the non-zero parameters of the expectational equations are treated as independent parameters to be estimated. Which parameters cannot be identified and why?

Looking at the bases of the null spaces for different parameterisations of the model, we find that the recurrent identification problem is that $\Gamma_{5}$ is not separately identifiable relative to $\sigma_{g^{*}}$. The last two columns of table 2.8 illustrates that the null space here usually only has one dimension, irrespective of the precise parameterisation. ${ }^{35}$

To avoid stochastic singularities we require the number of shocks to be greater than or equal to the number of observables. To be able to identify all 28 parameters of the foreign equations we require at least 4 observables (since 3 observables results in only 24 moments). If we match the number of observables to the number of shocks (5), then we have to exclude one observable out of our set of six. For the most part, excluding a single observable has no impact on the identification of the parameters. However, we find that excluding the foreign interest rate is problematic for identification, as it results in a loss of identification for one additional $\Gamma$ parameter.

We will examine which parameter(s) are dependent in a moment. First,

\footnotetext{
${ }^{34}$ The attentive reader will realise that we have not fully ignored the cross-equation restrictions in this case. In the 'model file' (see Schmitt-Grohé and Uribe 2012a) we have replaced combinations of structural parameters with $\Gamma$ parameters, but we have attached $\Gamma_{5}$ to $E_{t} \epsilon_{g^{*}, t+1}-\epsilon_{g^{*}, t}$.

${ }^{35}$ This result stems from the fundamental theorem of linear algebra / the rank-nullity theorem. For an $m \times n$ matrix the rank of the matrix plus the dimension of the null space will sum to $n$.
} 
Table 2.8: Identifying $\Gamma$ parameters for foreign equations: Minimum and maximum ranks for different observables

\begin{tabular}{lrrr} 
& \multicolumn{5}{c}{ Ranks } \\
Observables & Min & Max & \# Ranks $<$ Max \\
\hline$\pi^{*} \pi_{w}^{*} w^{*}$ & 13 & 13 & 0 \\
\hline$\pi_{w}^{*} r^{*} w^{*} n^{*}$ & 26 & 27 & 4 \\
$\pi^{*} r^{*} w^{*} n^{*}$ & 26 & 27 & 4 \\
$\pi^{*} \pi_{w}^{*} w^{*} n^{*}$ & 23 & 26 & 45 \\
$\pi^{*} \pi_{w}^{*} r^{*} n^{*}$ & 25 & 27 & 7 \\
$\pi^{*} \pi_{w}^{*} r^{*} w^{*}$ & 24 & 26 & 53 \\
$y^{*} r^{*} w^{*} n^{*}$ & 26 & 27 & 12 \\
$y^{*} \pi_{w}^{*} w^{*} n^{*}$ & 24 & 26 & 23 \\
$y^{*} \pi_{w}^{*} r^{*} n^{*}$ & 25 & 27 & 13 \\
$y^{*} \pi_{w}^{*} r^{*} w^{*}$ & 25 & 27 & 27 \\
$y^{*} \pi^{*} w^{*} n^{*}$ & 24 & 26 & 24 \\
$y^{*} \pi^{*} r^{*} n^{*}$ & 24 & 26 & 22 \\
$y^{*} \pi^{*} r^{*} w^{*}$ & 25 & 27 & 30 \\
$y^{*} \pi^{*} \pi_{w}^{*} n^{*}$ & 23 & 26 & 23 \\
$y^{*} \pi^{*} \pi_{w}^{*} w^{*}$ & 23 & 26 & 70 \\
$y^{*} \pi^{*} \pi_{w}^{*} r^{*}$ & 24 & 27 & 34 \\
\hline$\pi^{*} \pi_{w}^{*} r^{*} w^{*} n^{*}$ & 26 & 27 & 5 \\
$y^{*} \pi_{w}^{*} r^{*} w^{*} n^{*}$ & 26 & 27 & 4 \\
$y^{*} \pi^{*} r^{*} w^{*} n^{*}$ & 26 & 27 & 5 \\
$y^{*} \pi^{*} \pi_{w}^{*} w^{*} n^{*}$ & 24 & 26 & 28 \\
$y^{*} \pi^{*} \pi_{w}^{*} r^{*} n^{*}$ & 25 & 27 & 5 \\
$y^{*} \pi^{*} \pi_{w}^{*} r^{*} w^{*}$ & 25 & 27 & 27 \\
\hline$y^{*} \pi^{*} \pi_{w}^{*} r^{*} w^{*} n^{*}$ & 26 & 27 & \\
\hline
\end{tabular}

Note: This table presents ranks for a model that focuses on the elements of the $\Gamma$ parameters of the expectational equations. The ranks were computed at 10,000 points in the parameter space. The first column specifies the variables used as observables. The second and third columns indicate the minimum and the maximum rank found across the 10,000 parameter vectors. The ranks determine the number of elements that can be identified in the $\Gamma$ parameters. If the model were fully identified the ranks would be 28 . At least one $\Gamma$ parameter cannot be identified irrespective of the data set and the parameterisation. The last column counts the number of parameter vectors that result in sub-maximal ranks relative to the maximum ranks reported in the third column. This last column indicates that the vast majority of ranks coincide with the maximum rank reported in the third column. (In addition, 40 parameter vectors resulted in an indeterminate model.) 
however, it is worth dwelling on the computation of the rank of an arbitrary $m \times n$ matrix $A$. Trefethen and Bau (1997, ch. 5) suggest that the best numerical method for computing the rank of a matrix is to compute the reduced (thin) singular value decomposition, $A=\hat{U} \hat{\Sigma} V^{*}$, where $\hat{U}$ is $m \times n$, $V^{*}$ is $n \times n,{ }^{36}$ and $\hat{\Sigma}$ is an $n \times n$ matrix with a diagonal matrix of $r \leq$ $\min (n, m)$ singular values ordered from largest to smallest. If the matrix $A$ has rank $r$, then $\hat{\Sigma}$ will have $r$ non-zero values. To compute the rank of $A$ one simply counts the number of singular values greater than some "judiciously chosen tolerance" (p. 36). Golub and Loan (1996, pp. 260-261) argue that numerically computed singular values are well-approximated, subject to some machine error $\epsilon$, though computed $\hat{U}$ and $V^{*}$ matrices are not necessarily close to their exact counterparts. ${ }^{37}$ Computational inaccuracies related to $V^{*}$ are inconvenient since the $r+1$ to $n$ columns of $V$ correspond to a basis of the null space. Thus, while we will be able to determine the number of dependent columns reasonably accurately, it will be more difficult to compute the exact nature of the dependency.

The solution of a rational expectations model is obtained using computational methods and also results in a computational estimate of the Jacobian matrix. In principle it is possible to convert this numeric matrix into a symbolic matrix, though there is a substantial computational cost to doing so. To avoid this overhead, we employ computational estimates of the null space, using the Matlab null $\left(j, r^{\prime}\right)$ to compute the null-space. The ${ }^{\prime} r^{\prime}$ switch specifies the 'rational' basis associated with the reduced row echelon form. While computationally deprecated in favour of an orthonormal basis (as produced by the singular value decomposition), the rational basis makes it clear which columns of the Jacobian, and hence which parameters, are dependent (since the rows in the basis have non-zero coefficients). Because numerical errors can creep in from the digital approximation of numbers, we consider a value in the null space basis to be non-zero if its absolute value is greater than a threshold of $10^{-10}$.

We apply these computational techniques to compute null spaces for the

\footnotetext{
${ }^{36} V^{*}$ is the adjoint, the Hermitian conjugate of a complex matrix and the transpose of a real matrix. $V^{*}$ and $\hat{U}$ are unitary matrices with orthonormal columns.

${ }^{37}$ Note that Golub and Van Loan use 'hats' to denote computed values rather than the matrices of the reduced/thin singular value decomposition as done by Trefethen and Bau.
} 
sample $y^{*}, \pi^{*}, \pi_{w}^{*}, w^{*}, n^{*}$ (omitting $r^{*}$ ) for each of the 10,000 parameterisations. Although the null space computations are a bit variable across the parameter space, the additional identification problem are primarily associated with the coefficient on output in the Euler equation (which is associated with habit) and the coefficients on output and the growth rate of output in the monetary policy rule. As we note below, the cross-equation restrictions in the model ensure that these identification issues do not propagate through to the structural parameters in $\theta$.

\section{Identification of $\theta$}

We now examine whether the same identification problems arise when we directly focus on estimation of $\theta$ from the moments. To repeat, we simulate 10,000 parameter draws from the parameter space, drawing from the prior distributions reported in tables 2.6 and 2.7 using Sobol' sequences in conjunction with inverse probability integral transforms. It would be nice to increase the fineness with which the parameter space is explored, but this benefit is offset by the computational cost of increasing the number of parameter vectors in the analysis. Adapting Matlab code from Schmitt-Grohé and Uribe (2004), which uses the symbolic toolbox of Matlab, we solve the rational expectations model for its reduced form and compute the Jacobian of the variance-covariance matrix and first auto-covariance matrix of different samples of data. (See appendix 2.D.) Computing the derivatives symbolically mitigates a problem noted by Fernández-Villaverde et al. (2016, p. 654 ), namely it reduces the risk that numerical errors adversely affect the computation of the rank of the matrix.

Let us return now to the parameter draws for the DSGE model. If there is no unique solution to the rational expectations model - either because there is no solution at all or there is a multiplicity of solutions - we discard the parameter vector from the analysis. In practice, for the foreign/closed economy model, there are very few parameter draws that do not result in unique solutions (40 vectors out of 10,000).

We check identification using the variance matrix of observables and the first two autocovariance matrices. Let $\tilde{n}$ be the number of observables. Since the variance-covariance matrix is symmetric it has $\tilde{n}(\tilde{n}+2) / 2$ dis- 
tinct elements, while the two auto-covariance matrices need not be symmetric and thus each have $\tilde{n}^{2}$ distinct elements. Given that there are 23 parameters that we wish to estimate, we require at least $\tilde{n}=3$ observables $\left(\Rightarrow 2 \tilde{n}^{2}+\tilde{n}(\tilde{n}+1) / 2=24\right.$ moments $)$ to fully identify the model, given that we are not using steady-states to contribute to identification. In other words, $y_{t}$ in equation (2.3) has to be at least a $3 \times 1$ vector.

Using each possible combination of observable and each parameter vector drawn from the prior distributions, we compute the Jacobian matrix of the variance-covariance matrix and the first autocorrelation matrix. We then compute the ranks of these Jacobian matrices.

The identification analysis of the $\theta$ vector for the foreign block, reported in table 2.9 confirms the results that we have already found from looking at the two mappings $\Theta \rightarrow \boldsymbol{\Gamma}$ and $\boldsymbol{\Gamma} \rightarrow \mathbf{M}$. Using the null space, we find that $\eta^{*}$ cannot be identified and $\alpha_{w}^{*}$ and $\varepsilon_{w}^{*}$ are not separately identified. As there are fewer structural $\theta$ parameters than there are $\Gamma$ parameters, as few as 3 observables might suffice to identify the model parameters, but as usual it makes most sense for the number of observables to coincide with the number of shocks ( 5 in the foreign block). Once again, the rank of the Jacobian matrices is largely independent of the exact parameterisation of the model, confirmed by the last column of the table, which counts the number of instantiations of the model that result in sub-maximal ranks.

In contrast to the situation above where we considered the identification of the $\Gamma$ parameters, eliminating interest rates from our set of observables has no impact on the rank of the Jacobian computed with respect to $\theta$. The cross equation restrictions materially contribute to the identification of the underlying structural model, because the habit coefficient crops up in multiple $\Gamma$ elements.

\subsubsection{Identification of domestic parameters}

In this section we replicate the approach of the previous section but apply it to the domestic equations. To start, we consider again the mapping from the structural parameters in $\theta$ to the parameters in the $\Gamma$ matrices of the expectational equations. We specify the mapping using Matlab's Symbolic 
Table 2.9: Identifying $\theta$ parameters for foreign equations: Minimum and maximum ranks for different observables

\begin{tabular}{lrrr} 
& \multicolumn{5}{c}{ Ranks } & \\
Observables & Min & Max & \# Ranks $<$ Max \\
\hline$\pi_{w}^{*} r^{*} w^{*} n^{*}$ & 21 & 21 & 0 \\
$\pi^{*} r^{*} w^{*} n^{*}$ & 21 & 21 & 0 \\
$\pi^{*} \pi_{w}^{*} w^{*} n^{*}$ & 20 & 21 & 6 \\
$\pi^{*} \pi_{w}^{*} r^{*} n^{*}$ & 21 & 21 & 0 \\
$\pi^{*} \pi_{w}^{*} r^{*} w^{*}$ & 20 & 21 & 4 \\
$y^{*} r^{*} w^{*} n^{*}$ & 21 & 21 & 0 \\
$y^{*} \pi_{w}^{*} w^{*} n^{*}$ & 20 & 21 & 6 \\
$y^{*} \pi_{w}^{*} r^{*} n^{*}$ & 21 & 21 & 0 \\
$y^{*} \pi_{w}^{*} r^{*} w^{*}$ & 21 & 21 & 0 \\
$y^{*} \pi^{*} w^{*} n^{*}$ & 20 & 21 & 6 \\
$y^{*} \pi^{*} r^{*} n^{*}$ & 21 & 21 & 0 \\
$y^{*} \pi^{*} r^{*} w^{*}$ & 21 & 21 & 0 \\
$y^{*} \pi^{*} \pi_{w}^{*} n^{*}$ & 20 & 21 & 7 \\
$y^{*} \pi^{*} \pi_{w}^{*} w^{*}$ & 20 & 21 & 6 \\
$y^{*} \pi^{*} \pi_{w}^{*} r^{*}$ & 21 & 21 & 0 \\
\hline$\pi^{*} \pi_{w}^{*} r^{*} w^{*} n^{*}$ & 21 & 21 & 0 \\
$y^{*} \pi_{w}^{*} r^{*} w^{*} n^{*}$ & 21 & 21 & 0 \\
$y^{*} \pi^{*} r^{*} w^{*} n^{*}$ & 21 & 21 & 6 \\
$y^{*} \pi^{*} \pi_{w}^{*} w^{*} n^{*}$ & 20 & 21 & 0 \\
$y^{*} \pi^{*} \pi_{w}^{*} r^{*} n^{*}$ & 21 & 21 & 0 \\
$y^{*} \pi^{*} \pi_{w}^{*} r^{*} w^{*}$ & 21 & 21 & 21 \\
\hline$y^{*} \pi^{*} \pi_{w}^{*} r^{*} w^{*} n^{*}$ & 21 & 21 & 0 \\
\hline
\end{tabular}

Note: The first column specifies the variables used as observables. The second and third columns indicate the minimum and the maximum rank computed across the 10,000 parameter vectors. The ranks determine the number of elements that can be identified in the vector $\theta$. If the model were fully identified the ranks would be 23 . Thus, at least two parameters cannot be identified irrespective of the data set and the parameterisation. The last column counts the number of parameterisations with ranks that are less than that reported in the third column. (In addition, 40 parameter vectors resulted in an indeterminate model.) 
Toolbox and then compute the Jacobian with respect to the elements of $\theta$. We then compute a basis for the null space of this Jacobian matrix, illustrating which columns of the Jacobian are dependent. We find that the basis is two-dimensional. One of the domestic identification problems mirrors that of the foreign economy: $\varepsilon_{w}$ cannot be separately identified from $\alpha_{w}$. The second identification problem does not relate to $\eta^{*}$, which features in the domestic equations, but relates instead to $\varepsilon$, which is the elasticity of substitution between goods varieties. This $\varepsilon$ parameter does not affect any of the parameters in the expectational equations. This identification problem is commonly recognized. Justiniano and Preston (2010), for example, resolve it by calibrating $\varepsilon=8$.

We now explore whether domestic or foreign variables are more useful to achieve identification of the domestic parameters. Computationally, the analysis faces a similar problem to that of variable selection in Bayesian model averaging of linear regressions. Suppose that there are $n$ series that can be used as observables, then there are $\left(2^{n}\right)-1$ different ways of sampling from this set of data series (where the -1 means that we ignore the data sample that has zero columns). Intuitively, suppose that $X$ is a $T \times n$ matrix of data and specify an $n \times 1$ row vector of ones and zeros, where a 1 indicates that a column is included as an observable and a 0 indicates that it is not. For example [01011] indicates that the second, fourth and fifth columns are treated as observables, and the remaining two columns are not. This 5element vector can take binary values between 0 and 11111, where the latter number corresponds to 32 in base-10 terms. However, the model has 25 variables that could - theoretically at least - be used as observables. Since $2^{25}$ corresponds to roughly 33.5 million combinations it is infeasible to consider all such alternatives.

To reduce the dimensionality of the analysis we consider situations where the number of observables exactly equals the number of shocks. We also assume that some of the domestic and foreign variables are inherently unobservable, and thus exclude them from our analysis. In the open economy version of the model there are 12 shocks (seven domestic and five foreign), and we therefore select 12 observables.

We draw domestic observables from the following set: 
1. Gross domestic product

2. Consumer price inflation

3. Interest rates

4. The real exchange rate

5. Domestic consumption

6. Domestic consumption of home-produced goods

7. Domestic consumption of imports

8. The inflation rate for home-produced goods

9. The inflation rate for imported goods

10. The inflation rate for domestic wages

11. The terms of trade, and

12. Domestic hours worked.

And draw foreign observables from amongst the following:

1. Foreign output

2. Foreign inflation

3. Foreign wages

4. Foreign wage inflation

5. Foreign interest rates, and

6. Foreign hours worked.

Unfortunately, this number of combinations is still impractically large. The binomial coefficient for 18 choose 12 is $18 ! /(12 ! 6 !)=18,564$. To simplify the problem further, we assume that gross domestic product, consumer price inflation, interest rates and the exchange rate are always included as observables. That leaves us with 8 observable variables to choose from 14, which results in a mere 3,003 possible samples. Given that there are 12 domestic variables there is exactly one combination of observables that omits all foreign observables.

Space constraints preclude us from replicating table 2.9, reporting the ranks for each of these 3,003 samples, but we provide a small excerpt in table 2.10 as an illustration of some of the ranks from different samples. This excerpt shows that most samples result in the identification of all 29 parameters, but there is a reasonable degree of variation in the number of sub-maximal ranks that arise for different sets of observables. 
Table 2.10: Identifying $\Gamma$ parameters for foreign equations: Minimum and maximum ranks for different observables

Ranks

\begin{tabular}{llll} 
Observables & Min & Max & \#Ranks $<$ Max \\
\hline$\vdots$ & $\vdots$ & $\vdots$ & $\vdots$ \\
$c c_{f} \pi_{h} \pi_{f} \pi_{w} y^{*} w^{*} n^{*}$ & 29 & 29 & 0 \\
$c c_{f} \pi_{h} \pi_{f} \pi_{w} \mathrm{~s} y^{*} n^{*}$ & 29 & 29 & 0 \\
$c c_{f} \pi_{h} \pi_{f} \pi_{w} \mathrm{~s} w^{*} n^{*}$ & 29 & 29 & 0 \\
$c c_{f} \pi_{h} \pi_{f} \pi_{w} y^{*} r^{*} n^{*}$ & 29 & 29 & 0 \\
$c c_{f} \pi_{h} \pi_{f} \pi_{w} r^{*} w^{*} n^{*}$ & 29 & 29 & 0 \\
$c c_{f} \pi_{h} \pi_{f} \pi_{w} \mathrm{~s} r^{*} n^{*}$ & 29 & 29 & 0 \\
$c c_{f} \pi_{h} \pi_{f} \pi_{w} y^{*} \pi_{w}^{*} n^{*}$ & 29 & 29 & 0 \\
$c c_{f} \pi_{h} \pi_{f} \pi_{w} \pi_{w}^{*} w^{*} n^{*}$ & 29 & 29 & 0 \\
$c c_{f} \pi_{h} \pi_{f} \pi_{w} \mathrm{~s} \pi_{w}^{*} n^{*}$ & 29 & 29 & 0 \\
$c c_{f} \pi_{h} \pi_{f} \pi_{w} \pi_{w}^{*} r^{*} n^{*}$ & 29 & 29 & 0 \\
$c c_{f} \pi_{h} \pi_{f} y^{*} \pi^{*} w^{*} n^{*}$ & 27 & 29 & 217 \\
$c c_{f} \pi_{h} \pi_{f} \mathrm{~s} y^{*} \pi^{*} n^{*}$ & 27 & 29 & 249 \\
$c c_{f} \pi_{h} \pi_{f} \mathrm{~s} \pi^{*} w^{*} n^{*}$ & 27 & 29 & 235 \\
$c c_{f} \pi_{h} \pi_{f} y^{*} \pi^{*} r^{*} n^{*}$ & 27 & 29 & 227 \\
$c c_{f} \pi_{h} \pi_{f} \pi^{*} r^{*} w^{*} n^{*}$ & 27 & 29 & 216 \\
$c c_{f} \pi_{h} \pi_{f} \mathrm{~s} \pi^{*} r^{*} n^{*}$ & 27 & 29 & 249 \\
$c c_{f} \pi_{h} \pi_{f} y^{*} \pi^{*} \pi_{w}^{*} n^{*}$ & 27 & 29 & 211 \\
$c c_{f} \pi_{h} \pi_{f} \pi^{*} \pi_{w}^{*} w^{*} n^{*}$ & 27 & 29 & 208 \\
$c c_{f} \pi_{h} \pi_{f} \mathrm{~s} \pi^{*} \pi_{w}^{*} n^{*}$ & 27 & 29 & 232 \\
$c c_{f} \pi_{h} \pi_{f} \pi^{*} \pi_{w}^{*} r^{*} n^{*}$ & 27 & 29 & 213 \\
$\vdots$ & $\vdots$ & $\vdots$ & $\vdots$
\end{tabular}

Note: The first column specifies additional the variables used as observables. The four baseline variables, $y, r, \pi$ and $q$, are included in all combinations. The second and third columns indicate the minimum and the maximum rank computed across the 10,000 parameter vectors. The ranks determine the number of elements that can be identified in the vector $\theta$. The model is fully identified if the ranks are 29. The last column counts the number of parameterisations with ranks that are less than that reported in the third column. (In addition, 40 parameter vectors resulted in an indeterminate model.) 
We can make a few over-arching observations about these different sets of observables. First, however, we should note that we examine the identifiability of the following 29 domestic parameters: $\phi, \sigma, \alpha_{H}, \alpha_{F}, \alpha_{w}, \gamma_{H}, \gamma_{F}$, $\gamma_{w}, h, \tau, \eta, \theta_{\pi}, \theta_{y}, \theta_{\delta y}, \theta_{\delta e}, \theta_{r}, \rho_{a}, \rho_{g}, \rho_{w}, \rho_{c H}, \rho_{c F}, \rho_{r p}, \sigma_{a}, \sigma_{g}, \sigma_{c H}, \sigma_{c F}, \sigma_{w}$, $\sigma_{r} p, \sigma_{m}$. We exclude $\beta, \varepsilon, \varepsilon_{w}, \chi$ and $\rho_{m}$ from the identification analysis since all of these parameters are calibrated in Justiniano and Preston's priors.

When we examine the 3,003 combinations each with twelve observables, we find that each combination can identify all 29 of the elements of $\theta$ for the vast majority of the 10,000 instantiations (parameterisations) considered. We also find that there are many (924) combinations of observables that are impervious to the exact parameterisation of the model, identifying all 29 parameters across all instantiations, see figure 2.2. We find that 2,508/3, 003 combinations result in identification problems at 14 or fewer instantiations. In contrast, some combinations of observables suffer additional identification problems for $200-300$ of our 10,000 instantiations, as is evident from the sub-maximal ranks. According to the numerical ranks being computed, nine of the combinations of observables face some instantiations in which only 26/29 parameters can be identified.

Is the number of sub-maximal ranks correlated with the number of foreign variables included amongst the observables? figure 2.3 illustrates that a very low number of foreign variables (either zero or one) results in a very low number of additional identification problems. The figure plots a point for each combination of observables. The horizontal axis records the number of foreign variables in the combination and the vertical axis counts the number of times one or more parameters are unidentified across the 10, 000 instantiations. Each dimension (count) maps to the set of integers. When the number of foreign observables is 2 or more, there are $200-300$ instantiations that result in additional identification problems.

We determine which data have better identification properties by examining models with and without particular observable variables. We specify samples that contain the $i^{\text {th }}$ observable $y_{i}$ and identify the number of submaximal ranks for such samples and we contrast that number with the number of sub-maximal ranks when $y_{i}$ is not an observable. We undertake these contrasts for both the domestic and foreign variables. The results clearly 
Figure 2.2: Sub-maximal ranks by sample

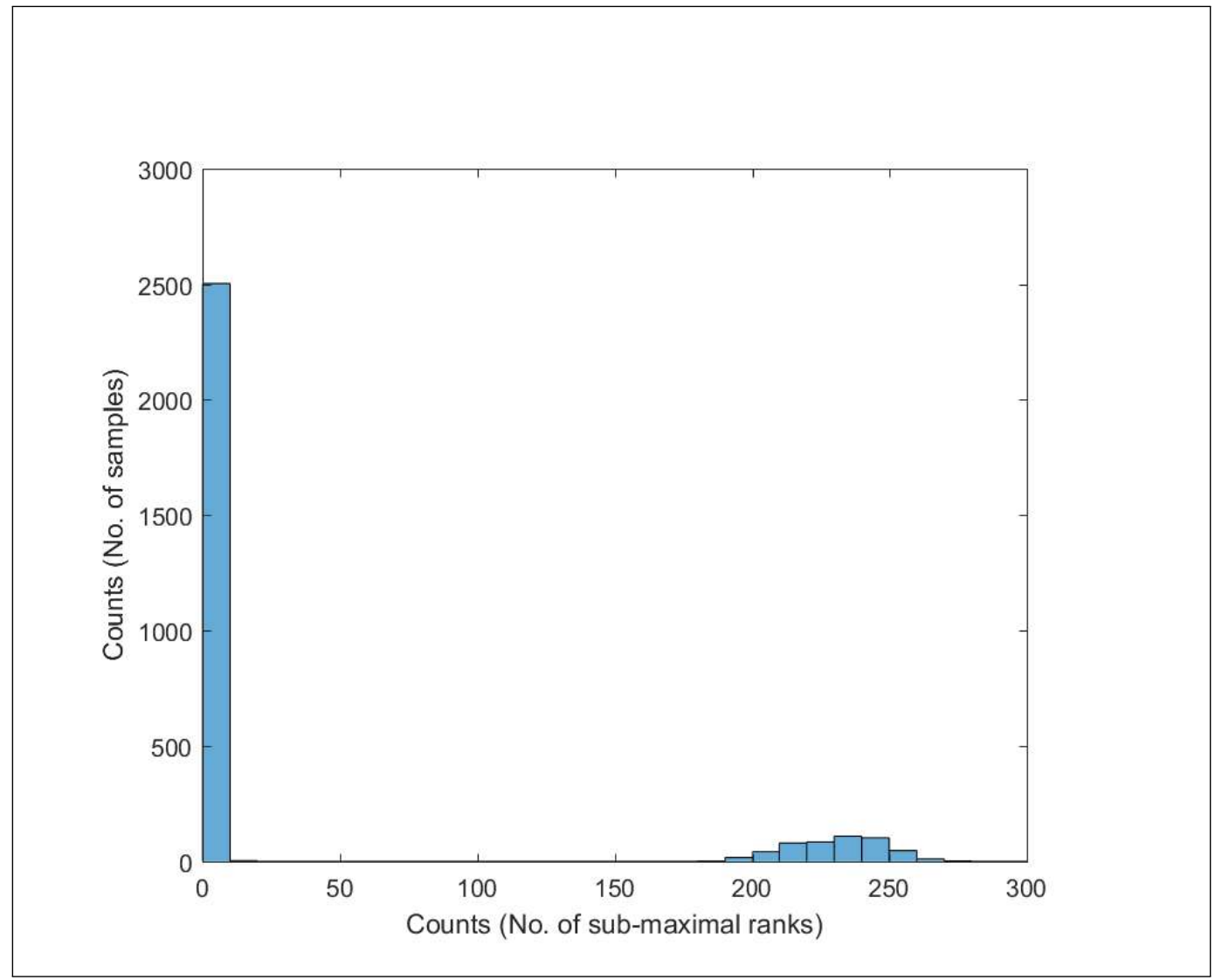

Note: This figure illustrates how many samples have sub-maximal ranks. The figure illustrates that roughly 2,500 of 3,003 combinations of observables successfully identify all 29 parameters with the exception of 14 or fewer instantiations. However, a non-trivial number of samples suffer from additional identification problems for around $2-3$ percent of instantiations (more exactly, $199-283$ instantiations out of 10,0000). 
Figure 2.3: Sub-maximal ranks vs the number of foreign variables included in the observables: Domestic parameters

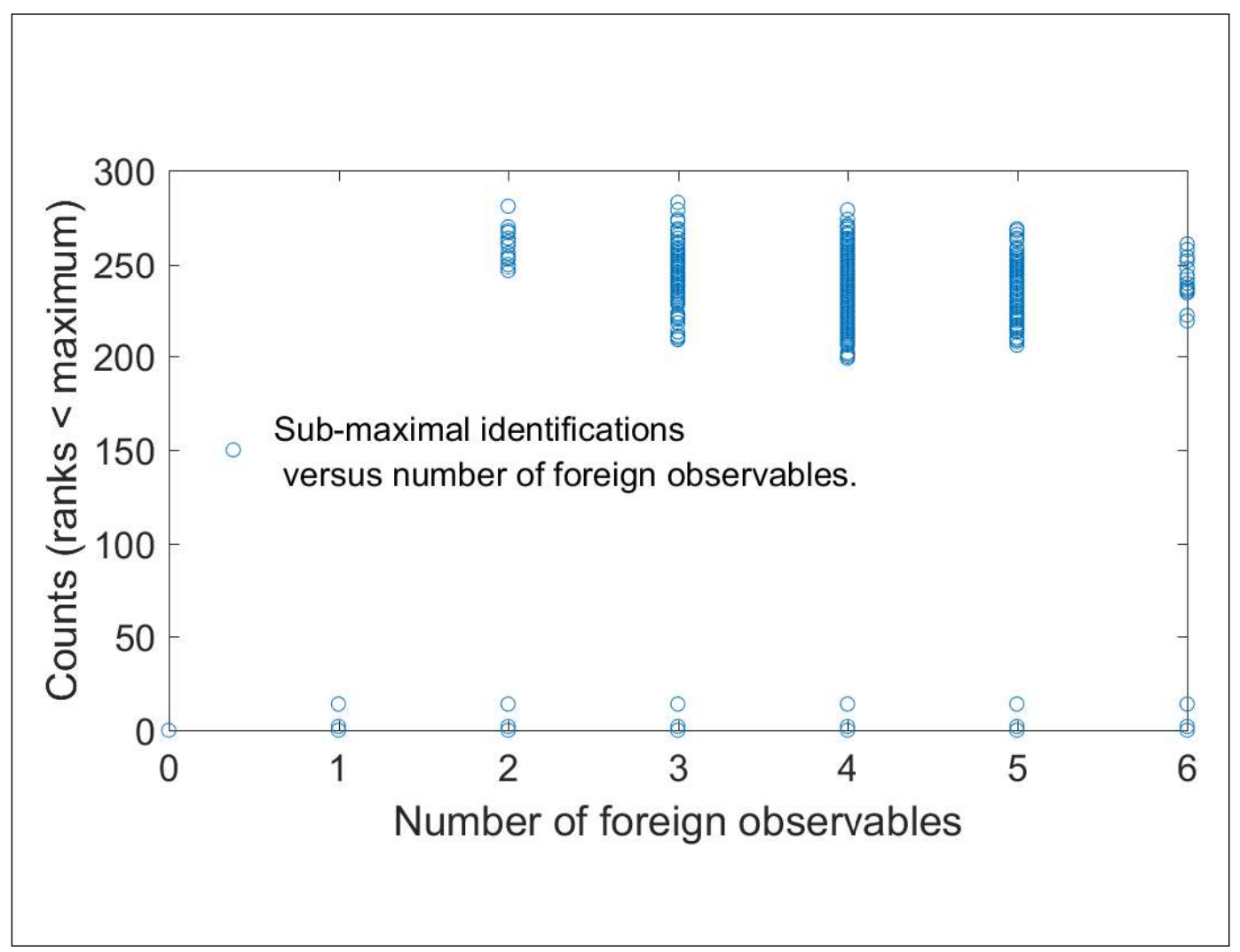

Note: This figure plots the number of sub-maximal ranks against the number of foreign variables included in the observables. There are 3,003 points, one for each combination. There is a single sample consisting solely of domestic variables. 
indicate that there is no single foreign variable that has a material impact on the frequency of sub-maximal ranks. Whether a foreign is included or excluded has little impact on the number of 'problem' instantiations.

The outlook is different for domestic variables. We find that including domestic labour market variables, either $\pi_{w}$ or $n$, is particularly valuable for eliminating these additional identification problems. In particular, we show that combinations that include domestic wage inflation or the number of hours worked have much better properties than combinations of observables that exclude both variables.

The benefit of labour market variables was not evident in our closed economy analysis of the foreign equations, reported in table 2.8, because the observable combinations we reported there always had labour market variables. In our examination of identification for closed economy parameters, three variables provided sufficient moments to estimate the structural parameters, at least in principle. For the sake of brevity we report only one 3-observable combination in that table, with price inflation, wage inflation and wages as observables. Those observables have very poor identification properties for the foreign parameters: only 13 structural parameters could be identified as compared to the more usual 21. Some data choices clearly matter for identification.

\subsubsection{Identification of foreign parameters from domes- tic data}

We look at the same combinations of 12 observables and consider whether the foreign parameters can also be locally identified. Figure 2.4 illustrates that many of the combinations of observables result in identification of 21 foreign parameters. (Each shade/colour corresponds to a particular combination of observables.) While many combinations enable the identification of 21 parameters, the figure also illustrates that a substantial number of instantiations have additional identification problems, with an additional one or two parameters no longer identified. These additional identification problems are much more common across the parameterisations than in our previous discussions. 
Table 2.11: Sub-maximal ranks across different combinations of observables Number of instantiations with ranks $<$ maximum

\begin{tabular}{lr}
\hline Minimum & 0.00 \\
Maximum & 278.00 \\
Mean & 43.78
\end{tabular}

Domestic variables

\begin{tabular}{lr}
\hline Include $c$ & 50.47 \\
Exclude $c$ & 34.85 \\
Include $c_{H}$ & 50.29 \\
Exclude $c_{H}$ & 35.10 \\
Include $c_{F}$ & 50.92 \\
Exclude $c_{F}$ & 34.25 \\
Include $\pi_{H}$ & 50.20 \\
Exclude $\pi_{H}$ & 35.20 \\
Include $\pi_{F}$ & 49.66 \\
Exclude $\pi_{F}$ & 35.94 \\
Include $\pi_{w}$ & 0.92 \\
Exclude $\pi_{w}$ & 100.91 \\
Include $s$ & 52.15 \\
Exclude $s$ & 32.61 \\
Include $n$ & 6.46 \\
Exclude $n$ & 93.53 \\
Include $\pi_{w}$ or $n$ & 5.05 \\
Exclude $\pi_{w}$ and $n$ & 239.97 \\
Foreign variables & \\
\hline Include $y^{*}$ & \\
Exclude $y^{*}$ & 50.56 \\
Include $\pi^{*}$ & 34.73 \\
Exclude $\pi^{*}$ & 50.66 \\
Include $\pi_{w}^{*}$ & 34.60 \\
Exclude $\pi_{w}^{*}$ & 49.65 \\
Include $r^{*}$ & 35.94 \\
Exclude $r^{*}$ & 50.70 \\
Include $w^{*}$ & 34.55 \\
Exclude $w^{*}$ & 49.83 \\
Include $n^{*}$ & 35.70 \\
Exclude $n^{*}$ & 50.39 \\
Eot I & 34.96 \\
\hline
\end{tabular}

Note: The table indicates the average number of 'problematic' instantiations associated with identification problems when a variable is included/excluded as an observable. The table indicates that observing wage inflation and/or hours worked decreases the number of instantiations with identification problems. Conversely, including foreign variables somewhat increases the number of problematic instantiations, presumably because it increases the probability of excluding domestic labour market variables. 
Figure 2.4: Histogram of ranks given 12 observables

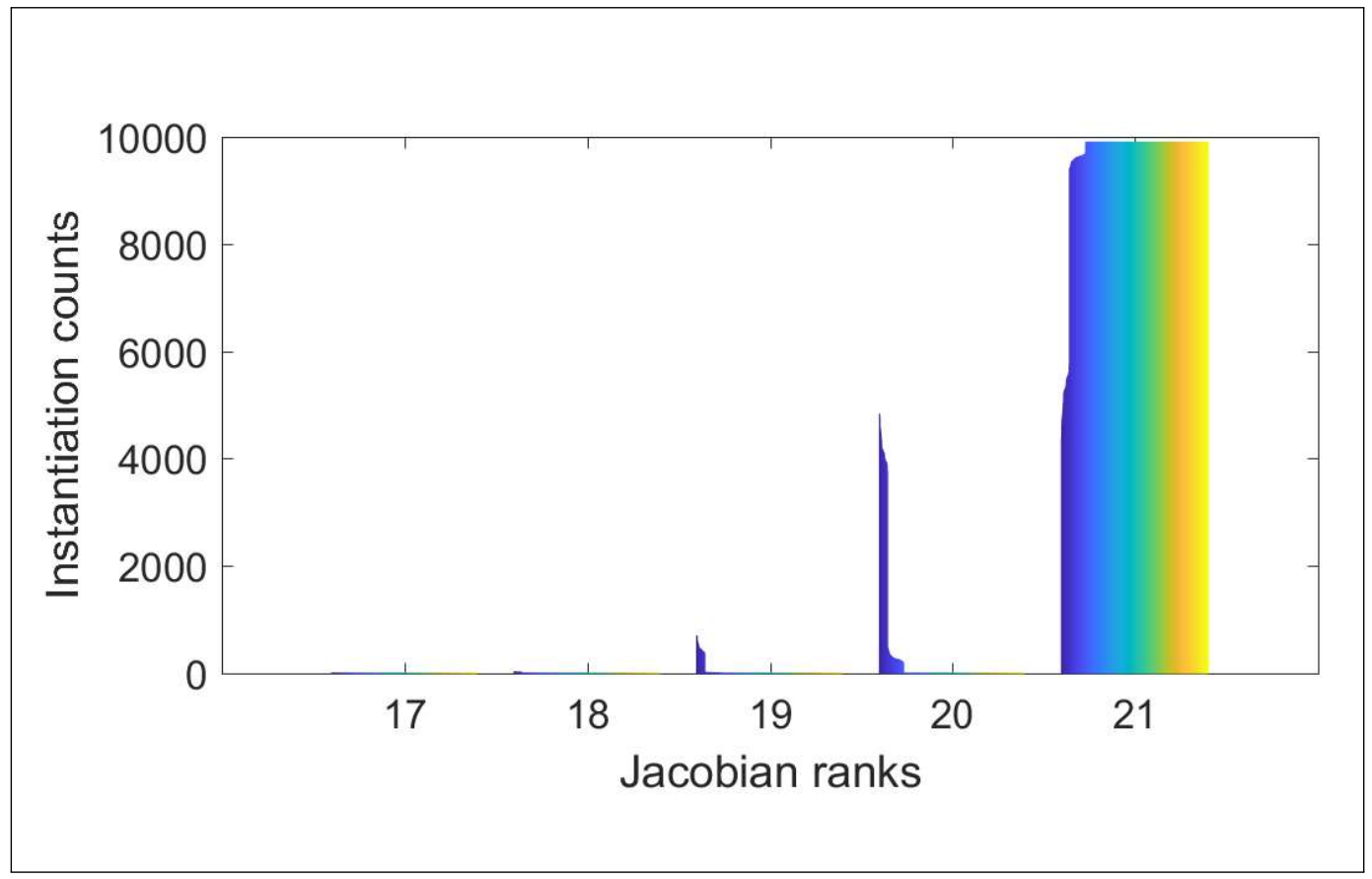

Note: This figure plots a histogram of ranks for each combination of 12 observables. The combinations were sorted to illustrate the differences in histograms more clearly. Each combination corresponds to a specific colour/shade. While many combinations identify 21/23 parameters, many combinations of observables result in additional identification problems. For some parameterisations of the model, in conjunction with particular observables, as few as 17 parameters are identified. 
Figure 2.5: Sub-maximal ranks vs the number of foreign variables included in the observables: Foreign parameters

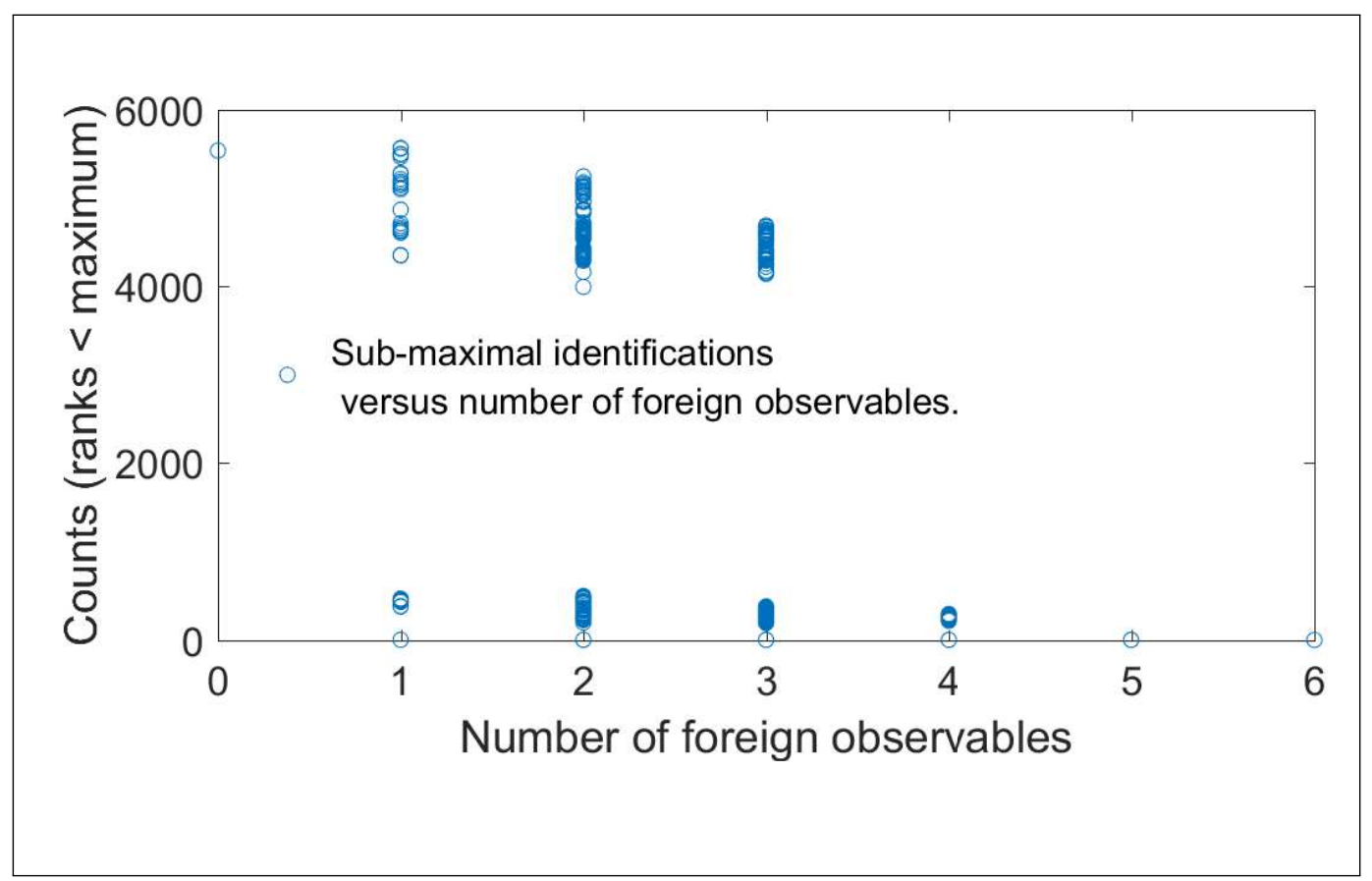

Note: This figure plots the number of sub-maximal ranks against the number of foreign variables included in the observables. There are thus 3,003 points, one for each combination of observables. There is a single sample consisting solely of domestic variables (zero foreign variables).

Figure 2.5 illustrates the counts of sub-maximal ranks against the number of foreign variables included in the combination of observables. This figure clearly illustrates that samples that have more foreign variables are more useful for identifying the foreign parameters. The figure also shows that domestic variables provide some degree of identification, because the dynamics and impulses from foreign variables still propagate to the domestic economy, at least to some degree.

Figure 2.6 demonstrates that the additional identification problems are much alleviated if $n^{*}$, the number of foreign hours worked, is included as an observable. A similar picture arises if we compare combinations of observables that include foreign wages, $w^{*}$. Including labour market data in the form of quantities or prices thus seems particularly valuable for the identification of foreign parameters. This figure reinforces the insight provided by table 2.11, that including labour market data is particularly useful for 
identification.

\subsubsection{What impact do autocorrelated errors have on identification?}

Pesaran (1987) and others note the importance of the dynamics of exogenous variables for identification in rational expectations models. In the context of our DSGE model a natural avenue to explore is the impact of autocorrelated shocks. The exogenous shocks that underpin business cycle fluctuations are typically modelled as autoregressive (or less commonly autoregressive-moving average) processes. Modelling the shocks as persistent processes is a pragmatic response to the fact that the intrinsic structure of our DSGE models fail to match the persistence of the data. What happens to identification when we turn off shock persistence? We take our baseline model but calibrate all of our autocorrelation terms, $\rho_{a}, \rho_{g}, \rho_{w}$, etc., to zero. We are then left with 23 domestic parameters to identify. Computing histograms across ranks we find that some combinations of observables identify 23 parameters and other combinations only identify 22 parameters. For a given combination of observables the same rank is achieved across all 10,000 points explored in the parameter space. To ascertain which observables influence identification we undertake the same contrast between combinations of observables that include variable $i$ and combinations that exclude variable $i$. The results are starkly illustrated in figure 2.7. Including $n$, the number of hours worked, enables the identification of 23 parameters, but only 22 parameters can be identified if $n$ is absent from the set of observables. In contrast, comparable figures for other inclusions/exclusions exhibit a mix of ranks in both cases. 
Figure 2.6: Histogram of ranks for foreign parameter identification with/without foreign hours worked

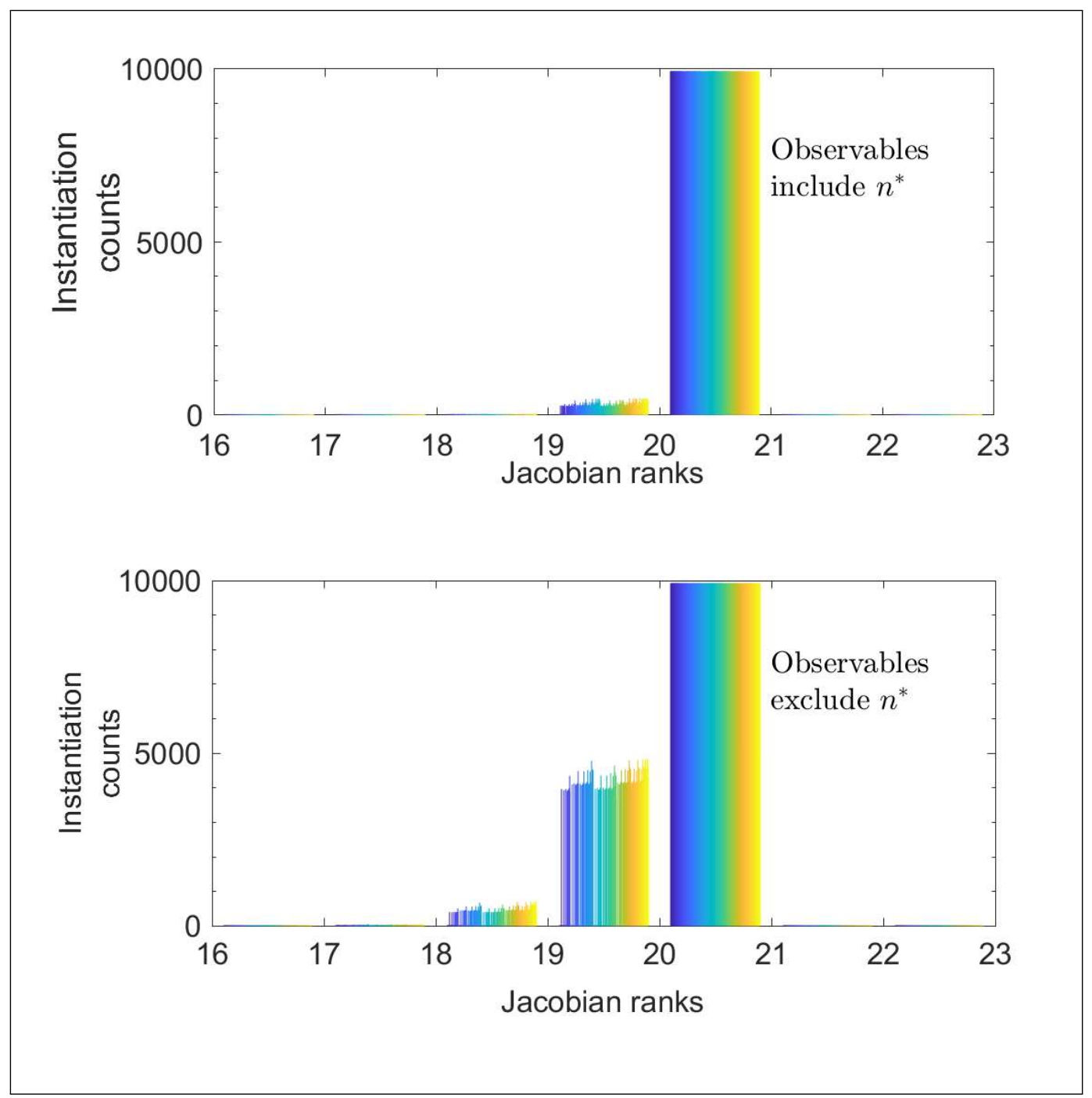

Note: This figure plots the histogram of ranks for different combinations of observables. The top panel illustrates histograms of ranks when $n^{*}$, foreign hours worked, is included as an observable. The bottom panel illustrates histograms of ranks when $n^{*}$ is excluded from the observables. The figure illustrates that observing $n^{*}$ results in markedly fewer identification problems. 
Figure 2.7: Histogram of ranks with/without labour market variables - IID shocks

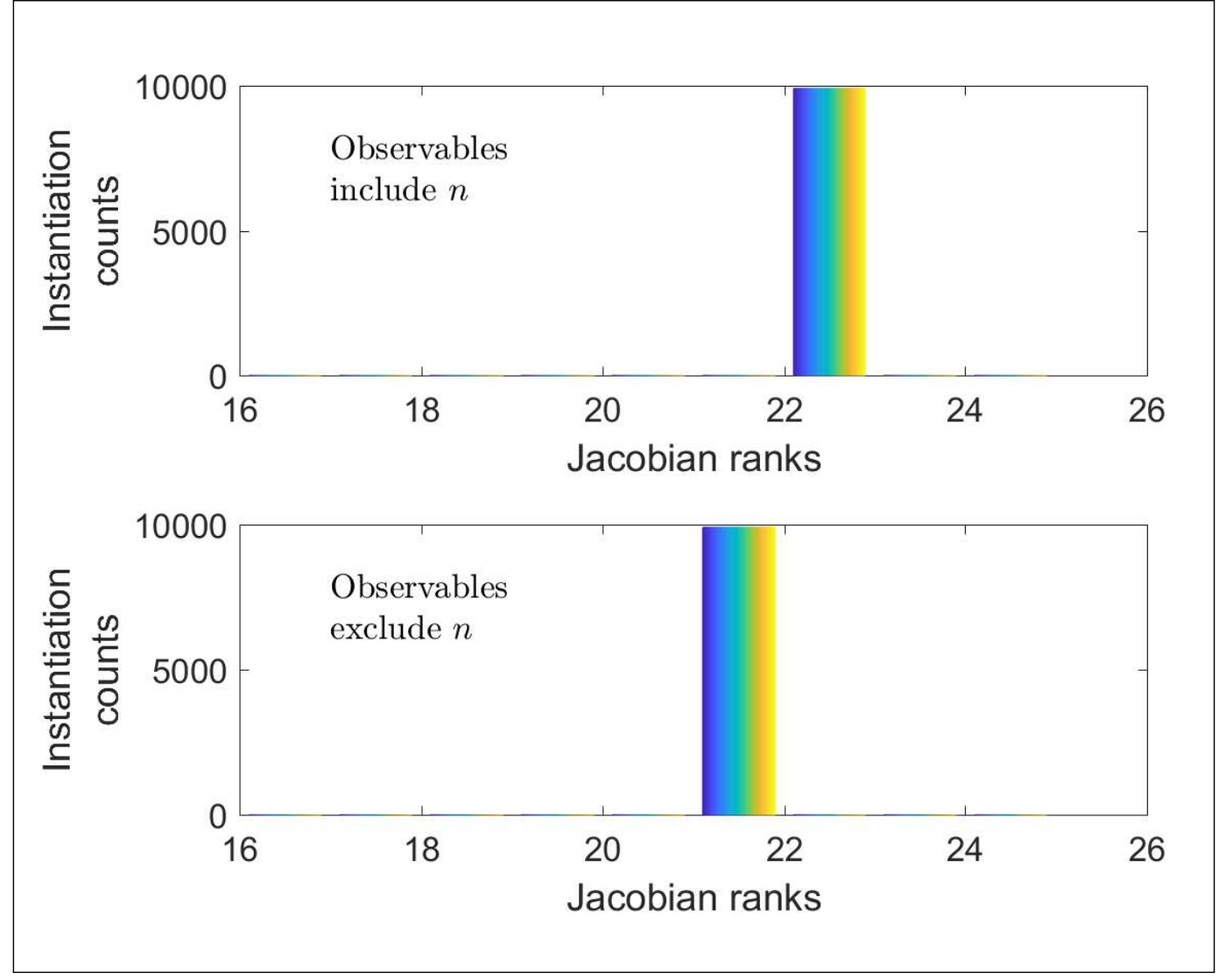

Note: This figure plots the histogram of ranks for different combinations of observables. The top panel illustrates histograms of ranks when domestic hours worked, $n$, is included as an observable. The bottom panel illustrates histograms of ranks when $n$ is excluded from the observables. The figure illustrates that observing $n$ is materially important for identifying one of the domestic parameters. 


\subsection{Conclusion}

In this chapter we explored whether the parameters of a small open economy DSGE model are identified. That is, can we correctly infer which parameterisation of the model generated the data if we know the properties of the data exactly?

A DSGE model is a system of expectational equations that arises from the optimising decisions of households, firms, and policy-makers. Once linearised, the system of equations is very similar to a system of simultaneous linear equations in the Cowles Commission tradition. DSGE models differ to simultaneous equations models in three main respects: i) $\Gamma$ parameters in the DSGE system are functions of underlying structural parameters $\theta$; ii) a DSGE model typically includes expectations of future variables; and iii) some DSGE variables are unobserved. Identification problems can arise because of each of these differences. We show that identification problems can arise in the mapping from the deep structural parameters $\theta$ that govern objectives and constraints to the $\Gamma$ parameters that instantiate the system of expectational equations.

One of the main contributions of the chapter is the development of a straightforward, easy-to-implement methodology that can be used to anticipate identification problems in the mapping from structural parameters $\theta$ to the $\Gamma$ parameters. By focusing explicitly on this mapping, we highlight identification problems and determine which structural parameters are interdependent and not separately identifiable.

Mechanically, we use the inverse function theorem and compute null spaces for the Jacobian matrix of a multivariate function to determine which, if any, parameters are interdependent. Since the mapping from a parameter vector $\theta$ to the $\Gamma$ matrices of the expectational equations is known, the Jacobian and null-space and ranks can be computed symbolically. For Bayesians, understanding these identification problems is important so that due care and attention can be directed to the priors for unidentified parameters, since data will not be fully informative about such parameters.

When the number of observed variables in a DSGE model is greater than the number of shocks a stochastic singularity arises in linear models, 
which results in an undefined likelihood. Undefined likelihoods arising from stochastic singularity pose a material problem for parameter estimation, both classically and for Bayesians optimising posterior beliefs about parameters. To avoid this problem, it is common to ensure that the number of observed variables is less than or equal to the number of shocks, by arbitrarily restricting the variables that are used as observables. We use the approach of Iskrev (2010b) to shed light on which variables should be used to estimate the model. We examine whether observed data moments change given local perturbations in the structural parameters. In particular, we examine whether changing the set of observables has material implications for local identification. In principle, identification may also depend on the parameterisation of the model. Thus, we examine identification at 10,000 points in the parameter space.

Our particular focus is on the use of foreign data. We examine whether foreign data are more useful than domestic variables in achieving model identification. Our results show that foreign data are not pre-eminently useful for the identification of domestic parameters. We start from a baseline of four domestic observables, $y, \pi, r$ and $q,{ }^{38}$ and then add 8 additional observables selected from a set of 8 domestic variables and 6 foreign variables, so that the number of observables coincides with the number of shocks. We find that at most points in the parameter space, all combinations of observables successfully identify the domestic parameters that we analyse (bar those subject to identification problems that arise in the mapping from $\theta$ to $\Gamma$ ).

We find that all feasible combinations of 12 variables are successful in locally identifying the parameters for the vast majority of parameterisations that we examine. However, some combinations of observables exhibit domestic identification problems for $2-3$ percent of the parameterisations, while other combinations of observables do not. We find that labour market variables - either hours worked or wage inflation - usefully augment the four benchmark variables in our analysis.

For countries with heavily diversified and/or idiosyncratic trade and financial relationships, constructing foreign data can be a challenging task, fraught with mis-measurement. Our results indicate that, if anything, do-

\footnotetext{
${ }^{38}$ Domestic output, inflation, interest rates, and the real exchange rate.
} 
mestic variables are more useful than foreign variables for achieving identification of the domestic parameters. For modellers in many small economies this finding will come as a relief, because it implies that one does not need to 'construct' foreign data to estimate small open economy models. However, it should be noted that foreign data make a constructive contribution to the identification of foreign parameters.

Our results provide modest support for a conclusion drawn by Iskrev and Ritto (2016), namely that identification can depend on the precise parameterisation of the model. However, our evidence suggests that identification properties are largely invariant to the parameterisation of the model. While it is possible for identification problems to crop up if too few observables are used or if the observables are too closely related to each, for the most part the identification properties of our model are the same across different combinations of observables. A mix of activity data, labour market data, and interest rates solves most identification problems, except those associated with mapping $\theta$ to $\Gamma$.

While the ranks of Jacobian matrices and the associated null spaces provide fundamental insight into the parameterisation of our models, they do not provide particularly sharp guidance as to which data series should be used as observables - many combinations of observables will identify the model. Thus, like Iskrev and Ritto (2016) and Canova et al. (2014), one needs to develop alternative criteria to guide the choice of observables. These criteria should reflect i) preferences (which moments are important to explain?); ii) beliefs about the accuracy or measurement quality of the data; and iii) beliefs about the veracity of our model for the data.

We began this chapter by quoting Fernandez-Villaverde: "Identification issues ought to be discussed in more detail in DSGE models, since they affect the conclusions we get from them." With the benefit of this chapter behind us, we think this claim should be amended. For the most part, we find that model parameters are identified irrespective of which data series are used to estimate the model. However, it may well be the case that the choice of the data affects the conclusions we draw from our models, reflecting the fact that our models are imperfect representations of the data. To challenge theory as much as possible, it makes eminent sense to condition our prior 
estimates with as much data as possible, preferably with series that relate to the underlying elements in our structural theories. Lastly, we should dive into the individual dimensions of the model to understand which additional data series are poorly captured by the model so that we may improve the accord of our models with the data. 


\section{Appendix}

\section{A Iskrev theorem 2}

The following theorem from Iskrev (2010b) provides a condition for local identification.

Theorem 2. Suppose that $m_{T}$ [A vectorized summary of $T$ autocovariance moments] is a continuously differentiable function of $\theta$ [a vector of structural parameters]. Then $\theta_{0}$ is locally identifiable if the Jacobian matrix $J(q):=$ $\partial m_{q} / \partial \theta^{\prime}$ has a full column rank at $\theta_{0}$ for $q \leq T$. This condition is both necessary and sufficient when $q=T$ if $u_{t}$ is normally distributed.

\section{B Inverse function theorem}

The following theorem is paraphrased from Rudin (1976, pp. 219-221), see particularly definition 9.20 and theorem 9.24.

Theorem 3 (Inverse function theorem). Suppose $f$ is a continuously differentiable mapping of an open set $E \subset \mathbb{R}^{n}$ into $\mathbb{R}^{n}, f^{\prime}(a)$ is invertible for some $a \in E$ and $b=f(a)$. Then

(a) there exist open sets $U$ and $V$ in $\mathbb{R}^{n}$ such that $a \in U, b \in V, f$ is one-to-one on $U$ and $f(U)=V$;

(b) if $g$ is the inverse of $f$ [which exists by (a)], defined in $V$ by $g(f(x))=x$ for $x \in U$, then $g$ is a continuously differentiable mapping on the set $V$. 


\section{C Identification of real-valued functions}

\section{C.1 Identifying function}

We replicate some of the definitions and lemmas of Kadane (1975, pp 176178) to note that there are mappings between 'identifying functions' (defined below). Kadane notes "[i]ntuitively such an identifying function carries with it all information about the identification of such functions." A natural implication is that the exact function used to identify the underlying parameter vector of a model is not material.

Definition 3 (Observational equivalence). Let $(\mathscr{X}, \mathscr{C})$ be a measurable space ( $\mathscr{X}$ is a set and $\mathscr{C}$ is $\sigma$-Algebra on $\mathscr{X}$ ) and let $P_{\theta}, \theta \in \Theta$ be a family of probability distributions on $(\mathscr{X}, \mathscr{C})$. Two parameter vectors $\theta, \theta^{\prime}$ are observationally equivalent if $P_{\theta}(A)=P_{\theta^{\prime}}(A)$ for all $A \in \mathscr{C}$.

Definition 4 (Identifying functions). An identifying function $T: \Theta \rightarrow \mathscr{T}$ is a function satisfying $\forall \theta, \theta^{\prime}$, such that $\theta$ is observationally equivalent to $\theta^{\prime} \Longleftrightarrow T(\theta)=T\left(\theta^{\prime}\right)$.

Given the above definitions, Kadane specifies the following lemma.

Lemma 1 (Identifying functions map). Let $f: \Theta \rightarrow \mathscr{S}$ and suppose $T$ : $\Theta \rightarrow \mathscr{T}$ is an identifying function. Then $f$ is identified $\Longleftrightarrow \exists$ a function $g: \mathscr{T} \rightarrow \mathscr{S}$ satisfying $f(\theta)=g(T(\theta))$.

To understand this result note that if the two functions are identifying then the structural parameter space $\Theta$ can be used as a bridge uniquely connecting elements $s \in \mathscr{S}$ to elements $t \in \mathscr{T}$ and vice-versa.

\section{C.2 Score of a Gaussian vector autoregression}

As noted in the text, the score - the vector of derivatives of the log-likelihood with respect to the parameters - is an obvious choice to use as an identifying function. Here we note that the score for a Gaussian process is a weighted function of the underlying variances and covariances of the data, the moments that we use to estimate the model. 
Let $Y=\left(\begin{array}{llll}y_{1} & y_{2} & \ldots & y_{T}\end{array}\right)^{\prime}$, where $y_{t}$ is an $n \times 1$ vector. Let $\Omega_{t}$ be the information set available at time $t$, consisting of $y_{t}, y_{t-1}, y_{t-2}, \ldots$ Suppose that $Y$ is a $\operatorname{VAR}(p)$ process. The prediction error decomposition of the likelihood of a vector is:

$$
\operatorname{Pr}(Y)=\operatorname{Pr}\left(y_{0}\right) \cdot \operatorname{Pr}\left(y_{1} \mid \Omega_{0}\right) \cdot \ldots \cdot \operatorname{Pr}\left(y_{t} \mid \Omega_{t-1}\right)
$$

Taking logs to form the log-likelihood, we have

$$
\log \operatorname{Pr}(Y)=\log \operatorname{Pr}\left(y_{0}\right)+\sum_{t=1}^{T} \log \operatorname{Pr}\left(y_{t} \mid \Omega_{t-1}\right)
$$

Define $X_{t-1}=\left(\begin{array}{llll}1 & y_{t-1}^{\prime} & \ldots & y_{t-p-1}^{\prime}\end{array}\right)^{\prime}$, a $1+p k \times 1$ vector. Then the $\operatorname{VAR}(\mathrm{p})$ can be represented as:

$$
y_{t}=B X_{t-1}+u_{t}
$$

with $B$ a $n \times 1+p k$ matrix. We assume that the vector of errors, also with dimension $n \times 1$, is multivariate Gaussian, $u_{t} \sim N(0, \Sigma)$. Then, given a suitable change-of-variable,

$$
\begin{aligned}
\log \operatorname{Pr}\left(y_{t} \mid \Omega_{t-1}\right) & =\log \operatorname{Pr}\left(y_{t} \mid X_{t-1}\right) \\
& =(2 \pi)^{-\frac{n}{2}}+|\Sigma|^{-\frac{1}{2}}-\frac{1}{2}\left(u_{t}\right)^{\prime} \Sigma^{-1}\left(u_{t}\right)^{\prime}
\end{aligned}
$$

given that $u_{t}$ has zero mean. Solving (2.10) for $u_{t}$ and substituting the answer into equation (2.11) and eliminating terms that are unrelated to $B$ we have:

$$
\begin{aligned}
\log \operatorname{Pr}\left(y_{t} \mid \Omega_{t-1}\right)= & \log \operatorname{Pr}\left(y_{t} \mid X_{t-1}\right) \\
= & -\frac{1}{2}\left(y_{t}-B X_{t}\right)^{\prime} \Sigma^{-1}\left(y_{t}-B X_{t}\right) \\
& + \text { unrelated terms }
\end{aligned}
$$

Noting that $\Sigma$ is symmetric and taking the derivative of (2.13) with respect 
to $B$ results in

$$
\frac{\partial \log \operatorname{Pr}\left(y_{t} \mid \Omega_{t-1}\right)}{\partial B}=\left(X_{t}\right)^{\prime} \Sigma^{-1}\left(y_{t}-B X_{t}\right)
$$

Given the definition of $X_{t}$ and $y_{t}$, the score of the likelihood with respect to the parameters in $B$ can be represented as a weighted average of variances and autocovariances of the data (summing across all $t=1, \ldots, T$, and scaling by $T$ ). See Lütkepohl (2006, sn. 3.4) for a rendition of the score using vec operators and Kronecker products. (The system of equations can be augmented with first order conditions with respect to the variance-covariance matrix of the error terms.)

\section{D DSGE solutions and identification}

We specify the DSGE model in the form used by Schmitt-Grohé and Uribe (2004), which uses policy function approximations. We closely follow SchmittGrohé and Uribe's notation and make no claim to originality in this section of the appendix. See Judd (1999) for a generic introduction to perturbation techniques, and Heer and Maußner (2009, ch. 2) for an introduction to perturbation techniques explicitly focused on DSGE models. We note that the notation in the following differs from that in the main text, which more closely follows Iskrev (2010b).

Suppose the system of equations from the DSGE model can be represented in the following form:

$$
\underset{t}{\mathrm{E}} f\left(y_{t+1}, y_{t}, x_{t+1}, x_{t}\right)=0
$$

where $\mathrm{E}_{t}$ is the expectation operator, conditional on the information available at time $t, y_{t}$ is the vector of non-predetermined variables at time $t$, and $x_{t}$ is the vector of predetermined variables. The system of equations is a mapping $f: \mathbb{R}^{n_{y}} \times \mathbb{R}^{n_{y}} \times \mathbb{R}^{n_{x}} \times \mathbb{R}^{n_{x}} \rightarrow \mathbb{R}^{n}$, where $n_{y}$ is the dimension of the vector $y_{t} ; n_{x}$ is the dimension of the vector $x_{t}$; and $n=n_{y}+n_{x}$. All vectors are assumed to be column vectors. The vector of predetermined (state) variables 
$x_{t}=\left(\begin{array}{ll}x_{1 t}^{\prime} & x_{2 t}^{\prime}\end{array}\right)^{\prime}$, where $x_{2 t}$ is a completely exogenous process

$$
x_{2 t+1}=\Lambda x_{2 t}+\tilde{\eta} \sigma \varepsilon_{t+1}
$$

where $\epsilon_{t}$ is an $n_{\varepsilon} \times 1$ vector of zero mean IID shocks with bounded support, and covariance matrix $\sigma \geq 0$ is a scalar and $\tilde{\eta}$ is an $n_{\varepsilon} \times n_{\varepsilon}$ matrix of parameters.

The first sub-component of the state vector, $x_{1 t}$ is a function of the entire state vector $x_{t}$. The solution to the model consists of two functions:

$$
\begin{gathered}
y_{t}=g\left(x_{t}, \sigma\right) \\
x_{t+1}=h\left(x_{t}, \sigma\right)+\eta \sigma \varepsilon_{t+1}
\end{gathered}
$$

And $\eta=\left(\begin{array}{cc}0^{\prime} & \tilde{\eta}^{\prime}\end{array}\right)^{\prime}$, with dimensions $n_{x} \times n_{\epsilon}$.

We seek a solution to the system equations via first (or second) order approximations of the policy function (see equations 2.18 and 2.19). Let $\bar{y}$ and $\bar{x}$ denote steady-state values. We compute a first order approximation around the non-stochastic steady-state (i.e. $\sigma=0)$. Substituting $(2.18)$ and (2.19) into (2.16), we have a function

$$
F(x, \sigma)=\underset{t}{\operatorname{E}} f\left(g\left(h(x, \sigma)+\eta \sigma \varepsilon^{\prime}, \sigma\right), g(x, \sigma), h(x, \sigma)+\eta \sigma \varepsilon^{\prime}, x\right)=0
$$

where a prime now indicates variables dated one period ahead. Since the function equals a constant, all the derivatives must leave the function unchanged: $F_{x^{k} \sigma^{j}}(x, \sigma)=0 \forall x, \sigma, j, k$, where this is the derivative of $F$ with respect to $x$ taken $k$ times and with respect to $\sigma$ taken $j$ times.

A first order approximation of the solution to the model provides two linear functions

$$
\begin{aligned}
& g(x, \sigma)=g(\bar{x}, 0)+g_{x}(\bar{x}, 0)(x-\bar{x})+g_{\sigma}(\bar{x}, 0) \sigma \\
& h(x, \sigma)=h(\bar{x}, 0)+h_{x}(\bar{x}, 0)(x-\bar{x})+h_{\sigma}(\bar{x}, 0) \sigma
\end{aligned}
$$

These linear functions can be inferred by taking the derivatives of $F$, as 
defined in equation (2.20), with respect to the elements of the vector $x$ and $\sigma$. Let $\left[f_{y^{\prime}}\right]_{\alpha}^{i}$ denote the derivative of the i-th equation with respect to the $\alpha$-th coefficient of $y^{\prime}$. (Again, remember that primes refer to periods onestep-ahead.) Then

$$
\begin{aligned}
{\left[F_{x}(\bar{x}, 0)\right]_{j}^{i} } & =\left[f_{y^{\prime}}\right]_{\alpha}^{i}\left[g_{x}\right]_{\beta}^{\alpha}\left[h_{x}\right]_{j}^{\beta}+\left[f_{y}\right]_{\alpha}^{i}\left[g_{x}\right]_{j}^{\alpha}+\left[f_{x^{\prime}}\right]_{\beta}^{i}\left[h_{x}\right]_{j}^{\beta}+\left[f_{x}\right]_{j}^{i} \\
& =0, \quad i=1, \ldots, n ; \quad j, \beta=1, \ldots, n_{x} ; \quad \alpha=1, \ldots, n_{y}
\end{aligned}
$$

for $i=1, \ldots, n ; j, \beta=1, \ldots, n_{x}$, and $\alpha=1, \ldots, n_{y}$. In (2.23), the derivatives of $F$ with respect to $y^{\prime}, x^{\prime}, x, y$ are evaluated at the steady state $\left(\begin{array}{llll}\bar{y} & \bar{y} & \bar{x} & \bar{x}\end{array}\right)$ and can be computed directly from the system of equations.

Equation (2.23) provides a set of quadratic equations, implicitly defining $h_{x}$ and $g_{x}$. The solution for $g_{x}$ and $h_{x}$ can be computed from (2.23) using the Schur decomposition of the system of equations. The matrices $-\left[f_{x^{\prime}}\right]_{\beta}^{i}$ and $-\left[f_{y^{\prime}}\right]_{\alpha}^{i}$ are concatenated together to form a matrix $A$ and $\left[f_{x}\right]_{j}^{i}$ and $\left[f_{y}\right]_{\alpha}^{i}$ are concatenated to form matrix $B$. We then compute the complex Schur decomposition of $A$ and $B$ and split the system into a set of equations with eigenvalues greater than one, which are recursed into the future, and equations that have eigenvalues less than one, which are 'solved backward'. The use of the Schur decomposition follows Klein (2000). See also Sims (2002) and DeJong and Dave (2007, ch. 2) for an accessible discussion. Analogous expressions can be derived by taking the derivative with respect to $\sigma$. Schmitt-Grohé and Uribe (2004) show that $h_{\sigma}=0$ and $g_{\sigma}=0$, so to first order the functions are unrelated to the size of the shock variances.

The solution to the reduced from can be used to compute the variances and autocovariances of the 'observed' variables. These moments can be thought of as a system of equations. Following Iskrev (2010b) we compute Hessians of these moments with respect to the structural parameters, which are derived from the partial derivatives of $f$ with respect to $y^{\prime}, y, x^{\prime}, x$. See Schmitt-Grohé and Uribe (2012a) for more explicit details. 


\section{E Composition of one-to-one functions is one-to-one}

Consider the following propositions.

Proposition A: $g \circ f$ is one-to-one

Proposition B: $f$ is one-to-one

We prove that $A \Rightarrow B$ by contradiction.

Proof. Suppose that $f$ is not one-to-one. Then there exists $\theta_{1} \neq \theta_{2}$ such that $f\left(\theta_{1}\right)=f\left(\theta_{2}\right)$. But by the composition function that implies $g \circ f\left(\theta_{1}\right)=$ gof $\left(\theta_{2}\right)$, and thus the composition is not one-to-one in contradiction to proposition A.

Consequently, if the DSGE model is to be identified then the mapping $f: \Theta \rightarrow \Gamma$ must be one-to-one. 


\section{Chapter 3}

\section{Skilled migration and business cycle dynamics}

\subsection{Introduction}

In recent years, migration flows have been large. Very large. These flows have been large in absolute numbers and large relative to non-migrant populations in destination countries. According to the United Nations, the world-wide stock of migrants increased by 17 percent between 2010 and 2017. ${ }^{1}$ In Western Europe migrant stocks rose by 18 percent, in the United States by 13 percent, in Canada by 16 percent and by 18 percent in Oceania. ${ }^{2}$ In contrast, overall population growth in high-income countries has been a paltry 3.8 percent, much slower than the growth of migrant populations. Even looking at the world as a whole, population growth has only been around 8.5 percent.

The economic causes and consequences of migration are complex and multi-dimensional, affecting both origin and destination countries. Kerr and Kerr (2011) and Nathan (2014) provide surveys that discuss various facets of migration, while Constant and Zimmermann (2013) and Chiswick and Miller (2015) provide handbooks on the topic. A particular focus of the literature has been on the effect of migration on the labour market (Borjas, 1999a; Dustmann et al., 2005; Borjas, 2014; Burstein et al., 2017). Much

\footnotetext{
${ }^{1}$ www.un.org/en/development/desa/population/migration/data/index.shtml, accessed 9 May 2018.

${ }^{2}$ See Peri (2016) for more cross-country detail, though for a slightly earlier period.
} 
of this analysis has a strong microeconometric focus, ${ }^{3}$ sometimes based on partial equilibrium models or models that exploit cross-country or regional variation. The macroeconomic consequences of migration, and in particular the general equilibrium business cycle consequences, are less well understood and have not been researched in much depth in the international literature. One notable exception is the work by Mandelman and Zlate (2012), which focuses on international risk sharing via migrants' remittances.

In this paper, we focus on the role of skilled migrants as a driver of the business cycle in countries-of-destination. How does migration affect the per capita level of gross domestic product (GDP) and its components? How does skilled migration affect the real exchange rate, and finally, do shocks to skilled migration drive the business cycle?

To address these questions, we develop and estimate a dynamic stochastic general equilibrium model for a small open economy that experiences net migration flows. We fit this model to the New Zealand economy, because of the availability of excellent migration data. All arrivals and departures in New Zealand are subject to reporting requirements and virtually all migrants arrive or depart by air, which provides a natural bottleneck for data collection. Migration flows into New Zealand have also been substantial in recent years, providing much-needed variation for econometric analysis. For example, net migration has increased working-age population in New Zealand by 1 percent in each of the three years from 2015-2017, and continues at a fast pace in 2018.

The key difference between migration flows and natural population increase is that migrants have pre-existing accumulations of financial and human capital, while babies enter the world with no capital balances. In our analysis we model migration allowing for human capital, but for simplicity we do not model stocks of financial capital. ${ }^{4}$

Our analysis enables us to determine the contribution of migration shocks to the business cycle. We illustrate that the skill level of migrants relative

\footnotetext{
${ }^{3}$ See, for example, the discussion paper series of the Centre for Research and Analysis of Migration.

${ }^{4}$ Skilled and other migrants entering New Zealand in 2004 and 2005 had on average about NZD36,000 worth of gross assets DOL (2009). Investor migrants, unsurprisingly, are required to bring in a larger stock of assets.
} 
to locals materially influences the dynamic impact of migration on the host economy. Borjas (1999a) notes that "the labour market impact of immigration hinges crucially on how the skills of migrants compare to those of natives in the host country". We show that relative skill levels also matter for variables such as consumption and investment, in addition to labour market variables such as hours worked and wages.

Migration shocks account for more of the volatility of the business cycle if migrants' level of human capital differs from that of locals. If migrants have a higher level of human capital than locals, the effects of migration are expansionary on a per capita basis and migration shocks can account for a large fraction of the volatility of GDP and its components. When migrants have the same human capital as locals, migration shocks account for only a small fraction of the overall volatility of GDP. While still expansionary on a per capita basis, this kind of migration causes much less volatility for the host economy.

The literature on the business cycle effects of migration can be traced back to Jerome (1926), who explored the implications of immigration into the United States in the early twentieth century. However, the modern literature on the macroeconomic effects of migration, using time series and structural macroeconomic models, is relatively sparse. Our work is related to Weiske (2017a,b), who looks at the macroeconomic effects of migration and population growth in the United States (US). Using constructed working-age net migration data for the United States in a vector autoregression, Weiske (2017a) finds that the short-run effects of migration are consistent with standard growth theory, i.e. real wages fall and investment increases. However, Weiske also finds that migration shocks make only a modest contribution to US business cycle dynamics. The latter result is not entirely surprising, since data from the Department of Homeland Security and the US Census Bureau suggest that the per annum migration rate for the United States has been below 1 percent since 1915 and, with two exceptions, has been below 0.4 percent since $1925 .^{5}$

\footnotetext{
${ }^{5}$ See https://www.dhs.gov/immigration-statistics/yearbook/2016/table1 and the Haver population series A111POPG10. These percentages are indicative since the immigration series are for fiscal years, and do not align perfectly with the Census numbers.
} 
Localised waves of migrants have been used as exogenous shocks to understand the effect of migration on wages, unemployment rates and house prices. Card (1990), for example, uses the 1980 Mariel boatlift of refugees from Cuba to Florida to examine the effects of migration on labour markets. The Mariel immigrants, relatively unskilled, increased the population of Miami by seven percent. Yet, according to Card, the influx had little effect on wages and unemployment rates overall. Borjas (2017), however, argues that the wage effects were substantial for the high school dropouts that were directly competing with the influx of unskilled migrants. The analytical framework developed below focuses on the average per capita effects of skilled migrant flows, not on the effect on specific types of labour grouped by skill. We will see, however, that relative skill-levels are particularly important for the macroeconomic consequences of migration shocks.

For some countries, the effects of migration shocks are substantial. Furlanetto and Robstad (2016), for example, use Norwegian data and find that positive migration shocks are expansionary and are a major driver of the dynamics of unemployment, though they are unimportant for house prices. ${ }^{6}$ Barrell et al. (2010) examine a particular facet of migration, namely the migration that occurred following the accession of ten Eastern European countries into the European Union, highlighting large flows into Ireland and the United Kingdom. In a Bundesbank working paper, Stähler (2017) examines the macro impact of refugees in Germany. In Stähler's model, refugees from the rest-of-the-world are absorbed only gradually into the labour force. Refugees initially increase output, via a demand channel, but the later dynamics depend on whether refugees accumulate more or less qualifications than locals.

In New Zealand, much of the macro literature on migration focuses on the housing market. ${ }^{7}$ Using a structural vector autoregression, Coleman and Landon-Lane (2007) find that migration has an extremely large impact on house prices, unlike the result reported for Norway by Furlanetto and Rob-

\footnotetext{
${ }^{6}$ Likewise, Saiz (2003) finds that the Mariel boatlift had little impact on house prices in Miami, though rents increased by substantially more than other metropolitan areas.

${ }^{7}$ Hodgson and Poot (2011) provide a summary of New Zealand research on migration between 2005 and 2010. Their synthesis focuses on labour market adjustment, but also discusses literature on housing, trade and tourism, fiscal impacts, and innovation.
} 
stad (2016). Stillman and Maré (2008) apply microeconometric techniques to New Zealand census and house sales data to examine the impact of population and migration on house prices at a local, disaggregated level; they find no impact of foreign-born migrants on local house prices, though returning New Zealanders seem to have a statistically significant impact. In contrast, McDonald (2013) investigates the composition of New Zealand migration and finds that constituent parts of net migration have different macro consequences: migrant arrivals are found to have more substantial impact on house prices than migrant departures and the citizenship of migrants also appears to have implications for the domestic (New Zealand) impact of migration. In a similar vein, Vehbi (2016) finds that the age-composition of migrants matters, with (presumably wealthier) 30-49 year old migrants having more substantial effects on consumption, house prices, rents, and residential investment than 17-29 year old migrants.

Having briefly described the literature we now turn to the specification of the structural model that we use to investigate the impact of migration flows on the business cycle.

\subsection{A model of migration in a small open economy}

We analyse the effects of migration shocks on business cycle dynamics using a dynamic stochastic general equilibrium (DSGE) model of a small open economy. The standard small open economy model is augmented with two features that have non-trivial implications for the economy's dynamic response to migration shocks. First, we allow for human capital accumulation, such that migration can affect not just the physical capital stock per head, but also the stock of human capital per capita. Importantly, the two forms of capital need not be affected by a migration shock in the same way. Second, we introduce a residential housing sector into the model. This addition allows us to analyse the effect of migration on residential real estate prices, and also sectoral labour flows. In other words, does migration cause labour to flow from the production of goods into the production of houses? We 
briefly discuss these two modelling choices in relation to the macroeconomic environment in New Zealand.

New Zealand's Immigration Act 2009 provides the current framework for migration into New Zealand. This legislation is augmented with regulations that specify application requirements for different visa categories. Visas are available for entrepreneurs, investors, skilled migrants, refugees, Pacific Islanders, and others. Of most note, in the context of our analysis, is the use of points-based criteria to rank applicants for many visa categories. Comparatively little use is made of visa ballots, ${ }^{8}$ such as those used to allocate 'Diversity Immigrant Visas' in the United States - green cards. In the ten and a half years from fiscal year 2007/8, roughly 463 thousand migrants have had visa applications approved by New Zealand immigration authorities. ${ }^{9}$ Some 263 thousand successful applicants (circa 57 percent of successful applicants) entered New Zealand as 'Skilled Migrants' or via investor, entrepreneur or other skill-related categories. A further 163 thousand migrants (35 percent) were approved for family-related reasons, around 15 thousand visas (3.2 percent) were granted for refugees, 16 thousand visas were approved for people from the Pacific (3.5 percent); and a little over 5 thousand people were provided visas for various other reasons (primarily by ministerial direction). While the measurement of human capital is clearly fraught, the importance of skilled, investor, and entrepreneurial migrants provides some support for the view that the 'average' migrant might have more human capital than the average domestic resident.

As mentioned above, we also explicitly model the housing sector. We incorporate housing into our analysis because housing is an important component of the capital stock, and demand for houses is directly and immediately affected by an increase in population - from migrants and residents alike. Residential investment is also one of the most volatile components of gross domestic product, contributing to business cycle fluctuations. Furthermore, construction is an important sector of the New Zealand labour market. According to the Quarterly Employment Survey, the proportion of full-time

\footnotetext{
${ }^{8}$ There are exceptions to this generalisation: Gibson et al. (2018) discuss the effects of Tongan migrants who enter into New Zealand via a lottery.

${ }^{9}$ See https://www.immigration.govt.nz/documents/statistics/ r1residencedecisionsbyfy.zip, downloaded 8 February 2018.
} 
equivalents employed in construction has increased from below 5 percent in the early 1990s to around 9 percent in the most recent data in 2017. The links between house values and consumption, and therefore aggregate demand, also receives continued emphasis in the monetary policy statements of the Reserve Bank of New Zealand, in part reflecting the fact that a substantial proportion of New Zealanders' domestic wealth is tied up in home ownership.

\subsubsection{Households}

In our model, households maximise expected utility defined over consumption, housing services, labour effort, and skill accumulation. The period utility function is

$$
U_{t}=\left(j_{t}^{c} \ln c_{t}+j_{t} \ln h_{t}-\frac{\phi_{0}}{1+\eta}\left(n_{t}+s_{t}\right)^{1+\eta}\right)
$$

where $c_{t}$ is consumption per capita, $h_{t}$ are housing services per capita, $j_{t}^{c}$ and $j_{t}$ are shocks that affects the utility agents derive from consumption and housing services, respectively. $n_{t}$ denotes working hours, and $s_{t}$ is training hours per capita. The final consumption good, $c_{t}$, consists of a domestically produced good, $c_{t}^{h}$, and an imported good, $c_{t}^{f}$. More precisely, the final good is defined as a constant elasticity of substitution (CES) aggregate:

$$
c_{t}=\left[v^{\frac{1}{\theta}}\left(c_{t}^{h}\right)^{\frac{\theta-1}{\theta}}+(1-v)^{\frac{1}{\theta}}\left(c_{t}^{f}\right)^{\frac{\theta-1}{\theta}}\right]^{\frac{\theta}{\theta-1}} .
$$

Here $\theta$ denotes the elasticity of substitution between the two types of goods and $v$ is the share of the domestically produced good in final consumption. The price index of the final good, $P_{t}$, is chosen to be the numeraire. Consequently, all other prices are expressed relative to the home final good. For example, the relative price of domestically produced goods, $p_{t}^{h}$, denotes the ratio $\frac{P_{t}^{h}}{P_{t}}$.

Households maximise expected utility subject to the flow budget con- 
straint:

$$
\begin{aligned}
c_{t}+p_{t}^{f} b_{t}+q_{t}^{H} h_{t}+p_{t}^{l} l_{t}= & \left(1+r_{t-1}\right) p_{t}^{f} \frac{N_{t-1}}{N_{t}} b_{t-1}+q_{t}^{H}\left(1-\delta_{h}\right) \frac{N_{t-1}}{N_{t}} h_{t-1} \\
& +w_{t} n_{t} \frac{N_{t-1}}{N_{t}} d_{t-1}+\left(p_{t}^{l}+R_{t}^{l}\right) \frac{N_{t-1}}{N_{t}} l_{t-1}+\pi_{t}
\end{aligned}
$$

Let us first note that the size of the working-age population at time $t$ is denoted by $N_{t}$. Expressing all variables in the model on a per capita basis implies that all carried-over stocks, such as housing, bonds, human capital and land in equation (3.3), are deflated by the term $\frac{N_{t-1}}{N_{t}}$, which is the inverse of the gross growth rate of working-age population. We implicitly assume that there is a great deal of risk sharing once migrants are assimilated into the local population.

In the budget constraint above, households consume goods, $c_{t}$, buy bonds that pay out in units of foreign-produced goods, $b_{t}$, buy housing services, $h_{t}$ at price $q_{t}^{H}$, and buy land, $l_{t}$ at price $p_{t}^{l}$. Households finance these expenditures through wage income, $w_{t} n_{t} d_{t}$ (reflecting both hours $n_{t}$ and human capital $\left.d_{t}\right)$; the return they receive from the bonds purchased in the previous period, $\left(1+r_{t-1}\right) b_{t-1}$; from the rental returns to their land holdings, $R_{t}^{l} l_{t-1}$; from selling the un-depreciated housing services purchased last period, $\left(1-\delta_{h}\right) h_{t-1}$; and through dividend income, $\pi_{t}$ that accrues to households as owners of the production sector. The stock of human capital, denoted $d_{t}$, evolves according to the following law of motion:

$$
d_{t}=\left(\frac{N_{t-1}}{N_{t}} d_{t-1} s_{t}\right)^{\phi_{s}} N_{t}^{2 \phi_{s}-1}+\left(1-\delta_{d}\right) \frac{N_{t-1}}{N_{t}} d_{t-1}
$$

where $\left(d_{t-1} s_{t}\right)^{\phi_{s}}$ denotes the production technology that turns effective time investment into human capital and $\delta_{d}$ denotes the depreciation rate of human capital. In modelling the accumulation of human capital we largely follow Kim and Lee (2007). ${ }^{10}$ Setting the parameter $\phi_{s}<1$ ensures that growth is exogenous. In our case with exogenous population growth we set $\phi_{s}=\frac{1}{2}$ to

\footnotetext{
${ }^{10} \mathrm{Kim}$ and Lee introduce human capital accumulation to explain why macro estimates of the intertemporal elasticity of substitution for labour supply are at odds with estimates from micro data. The macro elasticities are large to explain why labour supply fluctuations are large even though real wage variations are relatively small over the business cycle. The presence of human capital results in an implicit mis-measurement of real wages.
} 
rule out a scale effect from population growth.

\subsubsection{Household's first order conditions}

Equations (3.5) - (3.11) are the optimality conditions for consumption, hours worked, hours spent training, the accumulation of human capital, bonds, housing, and land. The marginal utility of consumption at time $t$ in these equations is denoted $\mu_{t}$ and the multiplier on the accumulation constraint for human capital is denoted $\lambda_{t}$.

$$
\begin{aligned}
& j_{t}^{c} / c_{t}-\mu_{t}=0 \\
& -\phi_{0}\left(n_{t}+s_{t}\right)^{\eta}+\mu_{t} w_{t} \frac{N_{t-1}}{N_{t}} d_{t-1}=0 \\
& -\phi_{0}\left(n_{t}+s_{t}\right)^{\eta}+\lambda_{t} \phi_{s} \frac{\left(\frac{N_{t-1}}{N_{t}} d_{t-1} s_{t}\right)^{\phi_{s}}}{s_{t}}=0 \\
& -\lambda_{t}+\mu_{t} w_{t} n_{t}+\beta \underset{t}{\mathrm{E}} \lambda_{t+1}\left[\phi_{s} \frac{\left(s_{t+1} \frac{N_{t}}{N_{t+1}} d_{t}\right)^{\phi_{s}}}{d_{t}}+\left(1-\delta_{d}\right) \frac{N_{t}}{N_{t+1}}\right]=0 \\
& -\mu_{t}+\beta \underset{t}{\mathrm{E}} \mu_{t+1} \frac{p_{t+1}^{f}}{p_{t}^{f}} \frac{N_{t}}{N_{t+1}}\left(1+r_{t}\right)=0 \\
& -q_{t}^{H}+j_{t} \frac{1}{\left(h_{t} \mu_{t}\right)}+\beta \underset{t}{\mathrm{E}} \frac{N_{t}}{N_{t+1}} \frac{\mu_{t+1}}{\mu_{t}}\left(\left(1-\delta_{h}\right) q_{t+1}^{H}\right)=0 \\
& -p_{t}^{l}+\beta \underset{t}{\mathrm{E}} \frac{N_{t}}{N_{t+1}} \frac{\mu_{t+1}}{\mu_{t}}\left(p_{t+1}^{l}+R_{t+1}^{l}\right)=0
\end{aligned}
$$

\subsubsection{Firms}

Households supply firms with effective labour, defined as $n_{t} d_{t-1} \frac{N_{t-1}}{N_{t}}=e n_{t}$, which is remunerated with the real wage $w_{t}$. Note that the opportunity cost of investing in human capital is borne exclusively by the household and not the firm. Households divide total effective labour, $e n_{t}$, between the goods producing sector, supplying $e n_{t}^{y}$ units of labour, and the construction sector, supplying $e n_{t}^{H}$ units of labour.

$$
e n_{t}=e n_{t}^{y}+e n_{t}^{H}
$$




\section{Goods sector}

Goods-producing firms produce a tradable good $y_{t}$ whose price in terms of the numeraire good is $p_{t}^{h}$. Firms maximise cash-flow defined as the difference between the value of output and expenditure on wages and investment, $x_{t}$ :

$$
\pi_{t}^{y}=p_{t}^{h} y_{t}-w_{t} e n_{t}^{y}-x_{t}
$$

subject to a production technology that combines effective labour and utilised capital:

$$
y_{t}=a_{t}\left(u_{t} \frac{N_{t-1}}{N_{t}} k_{t-1}\right)^{\alpha}\left(e n_{t}^{y}\right)^{1-\alpha} .
$$

The usual law of motion of the capital stock is defined as:

$$
k_{t}=\left(1-\delta\left(u_{t}\right)\right) k_{t-1} \frac{N_{t-1}}{N_{t}}+a_{t}^{i} \iota\left(x_{t} / x_{t-1}\right)
$$

where the depreciation rate $\delta()$ is a function of the utilisation rate, $u_{t}$. The function $\iota\left(x_{t} / x_{t-1}\right)$ represents investment adjustment costs, as per Christiano et al. (2005), and $a^{i}$ denotes a shock to the marginal efficiency of investment (MEI). Investment $x_{t}$ is denominated in terms of the final good, with the numeraire price of 1 . The standard optimality condition for capital, investment, and utilisation are:

$$
\begin{gathered}
q_{t}=\underset{t}{\mathrm{E}} \beta \frac{N_{t}}{N_{t+1}} \frac{\mu_{t+1}}{\mu_{t}}\left(p_{t+1}^{h} \frac{\partial y_{t+1}}{\partial k_{t}}+q_{t+1}\left(1-\delta\left(u_{t+1}\right)\right)\right) \\
1 / a_{t}^{i}=q_{t} \frac{\partial \iota\left(x_{t}, x_{t-1}\right)}{\partial x_{t}}+\beta \underset{t}{\mathrm{E}}\left(\frac{\mu_{t+1}}{\mu_{t}} q_{t+1} \frac{\partial \iota\left(x_{t+1}, x_{t}\right)}{\partial x_{t}}\right) \\
\alpha p_{t}^{h} \frac{y_{t}}{u_{t}}=q_{t} \delta^{\prime}\left(u_{t}\right) k_{t}
\end{gathered}
$$

\section{Construction sector}

Our housing and construction sector is based on Iacoviello (2005). Housing stock is built using effective labour, land and home-produced intermediate goods, $m_{t}$. Profits in the construction sector at time $t$ are defined as $\pi_{t}^{H}$, 
with

$$
\pi_{t}^{H}=q_{t}^{H} H_{t}-w_{t} e n_{t}^{H}-R_{t}^{l} l_{t-1}-p_{t}^{h} m_{t}
$$

where $q_{t}^{H}$ denotes the price of newly built housing stock. Labour mobility across sectors ensures that builders face the same wage costs as do goods producing firms, $w_{t}$. The rental rate of land faced by the construction sector is denoted by $R_{t}^{l}$. Profits are maximised subject to the following production technology for new housing:

$$
H_{t}=a_{t}^{H}\left(\frac{N_{t-1}}{N_{t}} l_{t-1}\right)^{\xi_{l}}\left(e n_{t}^{H}\right)^{1-\xi_{l}-\xi_{m}} m_{t}^{\xi_{m}}
$$

The production of houses is, like the production of goods, subject to an $\operatorname{AR}(1)$ technology shock, $a_{t}^{H}$. The construction firm maximises profits by choosing effective labour, land and intermediate inputs optimally:

$$
\begin{gathered}
\left(1-\xi_{l}-\xi_{m}\right) q_{t}^{H} \frac{H_{t}}{e n_{t}^{H}}=w_{t} \\
\xi_{l} q_{t}^{H} \frac{H_{t}}{l_{t-1}}=R_{t}^{l} \\
\xi_{m} q_{t}^{H} \frac{H_{t}}{m_{t}}=p_{t}^{h}
\end{gathered}
$$

Every period, households sell their un-depreciated housing stock and purchase new homes with the proceeds. Market clearing implies that the supply of new houses equals the net increase in the housing stock.

$$
H_{t}=h_{t}-\left(1-\delta_{h}\right) \frac{N_{t-1}}{N_{t}} h_{t-1}
$$

The total amount of land in the economy is fixed, but as the population grows the supply of land per household diminishes. A temporary increase in migration, or indeed just natural population growth, would imply an ever decreasing amount of land per household. From a modelling perspective, the steady state around which we are linearising the model would therefore not be deterministic. We get around this problem by assuming that land is re-zoned for building purposes as the population grows. As the supply of building land grows along with the population, the supply of land per 
household remains constant:

$$
l_{t}=1
$$

\subsubsection{Current account}

Having described the household and production sectors above, this section presents the final equations needed to close the model. Market clearing in the home-produced goods market is described by equation (3.26):

$$
y_{t}-m_{t}=v\left(p_{t}^{h}\right)^{-\theta}\left(c_{t}+x_{t}\right)+e x_{t}^{h}
$$

The home-produced good is used in the production of the domestically consumed final good and the final investment good, which is used in domestic production, and is also exported and used as an intermediate input in construction. Export demand from abroad is assumed to be of the form:

$$
e x_{t}^{h}=v^{*}\left(\frac{r e r_{t}}{p_{t}^{h}}\right)^{\theta^{*}} y_{t}^{*}
$$

with $y_{t}^{*}$ denoting total foreign demand for the domestic good. Substituting the market clearing conditions from the goods and labour markets into the household budget constraints yields the economy-wide current account equation:

$$
y_{t}=c_{t}+x_{t}+m_{t}+p_{t}^{f} b_{t}-p_{t}^{f}\left(1+r_{t-1}\right) \frac{N_{t-1}}{N_{t}} b_{t-1}
$$

Finally, we close the model by introducing a debt elastic interest rate premium that allows for small deviations of the domestic real interest rate from the world rate when the domestic net foreign asset position deviates from its steady state level. This eliminates the unit-root in bond holdings:

$$
1+r_{t}=\left(1+r_{t}^{*}\right) e^{-\phi_{b}\left(b_{t}-\bar{b}\right)}
$$

Details of the steady state can be found in section 3.A of the appendix to this chapter. 


\subsubsection{Driving processes}

The model economy is driven by seven shocks each of which is an $\operatorname{AR}(1)$ process:

$$
\begin{aligned}
a_{t} & =\rho_{a} a_{t-1}+\epsilon_{a t} \\
a_{t}^{H} & =\rho_{h} a_{t-1}^{H}+\epsilon_{h t} \\
j_{t} & =\rho_{j} j_{t-1}+\epsilon_{j t} \\
j_{t}^{c} & =\rho_{j c} j_{t-1}^{c}+\epsilon_{j c t} \\
a_{t}^{i} & =\rho_{i} a_{t-1}^{i}+\epsilon_{i t} \\
y_{t}^{*} & =\rho_{y *} y_{t-1}^{*}+\epsilon_{y * t} \\
v_{t} & =\rho_{v} v_{t-1}+\epsilon_{v t}
\end{aligned}
$$

Equations (3.30) and (3.31) are total factor productivity processes in goods production and construction, respectively. Equations (3.32) and (3.33) represent preference shocks for housing and consumption, while (3.34) denotes the MEI shock process. World output and net migration follow the processes denoted in (3.35) and (3.36). Specifically, the migration process is defined as $v_{t} \equiv \ln \left(N_{t} / N_{t-1}\right)$.

Modelling migration as an exogenous process is a simplifying assumption, with some empirical support in our reduced form analysis, depending on how the model is specified. The literature also provides a degree of support for this assumption. Mitchell et al. (2011) find that simple autoregressive models can provide more accurate forecasts of migration in the United Kingdom than models that include economic or policy factors - in part because policy factors are hard to forecast. ${ }^{11}$

\footnotetext{
${ }^{11}$ Conversely, theory emphasizes that migration should be endogenous to domestic and foreign conditions, see for example Borjas (1999a). Alternative empirical methodologies do uncover endogenous effects at some frequencies: Mayda (2010), for example, conducts a panel data analysis based on annual data from 14 developed countries and finds that 'pull' factors in destination economies, such as relative income levels, do affect migration flows, though 'push' factors have only small effects.
} 
Table 3.1: Variables - summary Domestic

Domestic continued

\begin{tabular}{|c|c|c|c|}
\hline$y_{t}$ & Output (tradable) & $p_{t}^{h}$ & Price of tradables \\
\hline$h_{t}$ & Flow of housing services & $p_{t}^{f}$ & Price of imports \\
\hline$N_{t}$ & Total population & $w_{t}$ & Wages \\
\hline$n_{t}$ & Hours worked & $q_{t}$ & Price of capital \\
\hline$e n_{t}$ & Effective hours & $q_{t}^{H}$ & Price of housing \\
\hline$e n_{t}^{y}$ & Effective hours tradables & $p_{t}^{l}$ & Price of land \\
\hline$e n_{t}^{H}$ & Effective hours housing & $R_{t}^{l}$ & Rental return on land \\
\hline$s_{t}$ & Training hours & $\operatorname{rer}_{t}$ & Real exchange rate \\
\hline$d_{t}$ & Stock of human capital & & \\
\hline$c_{t}$ & ${ }^{\dagger} \mathrm{CES}$-aggregate of consumption & \multicolumn{2}{|c|}{ Exogenous processes } \\
\hline$c_{t}^{h}$ & Consumption of tradables & $a_{t}$ & Technology shock \\
\hline$c_{t}^{f}$ & Consumption of foreign goods & $a_{t}^{H}$ & Housing technology shock \\
\hline$b_{t}$ & Bond holdings (assets) & $j_{t}$ & Housing preference shock \\
\hline$l_{t}$ & Land per capita & $j_{t}^{c}$ & Consumption preference shock \\
\hline$r_{t}$ & Interest rate & $a_{t}^{i}$ & ${ }^{\ddagger} \mathrm{MEI}$ shock \\
\hline$u_{t}$ & Utilisation & $y_{t}^{*}$ & Foreign income \\
\hline$k_{t}$ & Capital & $v_{t}$ & Migration shock \\
\hline$x_{t}$ & Investment & & \\
\hline$m_{t}$ & Intermediate goods & \multicolumn{2}{|c|}{ Foreign } \\
\hline$\pi_{t}$ & Dividend income & $r_{t}^{*}$ & Foreign interest rate \\
\hline$e x_{t}^{h}$ & Exports & $y_{t}^{*}$ & Foreign income (see also above) \\
\hline
\end{tabular}

Notes: $\dagger$ CES $=$ Constant elasticity of substitution. $\ddagger$ MEI $=$ Marginal efficiency of investment.

\subsubsection{Model description in brief}

To round out the presentation of the model we briefly summarise the notation for our variables in table 3.1. The parameters, collated with their priors and posteriors, can be found in table 3.5.

\subsection{Migration versus population growth}

What is the key difference between migration and population growth? In the model, the main effect of both migration and population growth is to dilute existing stocks of capital, housing, human capital, and net foreign assets on 
a per capita basis. What differentiates migration from population growth is that for human capital, at the very least, migration need not reduce the per capita level of the relevant stock. Indeed, migration may even raise the average human capital in the economy.

To illustrate the effect of migrants on the stock of human capital consider the log-linearised evolution of $d_{t}$ over time when migrants arrive with no human capital:

$$
\hat{d}_{t}=\phi_{s} \delta_{d}\left[\hat{d}_{t-1}-v_{t}+\hat{s}_{t}\right]+\left(1-\delta_{d}\right)\left[\hat{d}_{t-1}-v_{t}\right]
$$

Unskilled migration reduces the per capita stock of human capital in the economy. Skilled migration in the model would imply that the evolution of human capital per head is not affected by migration. We thus amend the equation above as:

$$
\hat{d}_{t}=\phi_{s} \delta_{d}\left[\hat{d}_{t-1}-(1-\chi) v_{t}+\hat{s}_{t}\right]+\left(1-\delta_{d}\right)\left[\hat{d}_{t-1}-(1-\chi) v_{t}\right]
$$

where $\chi$ is strictly positive and takes the value of 1 when migrants possess the same level of human capital as natives, or greater than 1 when migrants have a higher average level of human capital.

\subsection{Estimation strategy}

The principal aim of our model is to shed light on the short-run macroeconomic effects of migration shocks and assess their contribution to the dynamics of the business cycle. To this end, we implement a Bayesian estimation procedure. We estimate the model using macroeconomic data for New Zealand, an economy that has experienced both large and volatile migration flows in recent decades. In addition, New Zealand is one of the very few countries for which reliable data on working-age net migration is available.

We focus our analysis on 'permanent and long-term' (PLT) arrivals and departures. PLT arrivals are people arriving for a stay of 12 months or more, including New Zealanders returning after an absence overseas of 12 months or more. Conversely, PLT departures are New Zealanders departing for 12 
months or more and migrants leaving after a stay of 12 months or more in New Zealand. Net migration figures in New Zealand are often decomposed into net migration between New Zealand and Australia and net migration relative to the rest of the world. Australian and New Zealand citizens largely have freedom of movement between the two countries, including the right to work. In Figure 3.1, net migration between New Zealand and Australia is summarised by the chequered (blue) bars and that between New Zealand and the rest of the world by the solid (red) bars. ${ }^{12}$ Over the sample, there was negative net migration between New Zealand and Australia, offset by positive migration between the rest of the world and New Zealand. Since about 2014, net emigration to Australia has dried up, while net migration into New Zealand from the rest of the world has increased. As a result, annual net migration has risen to about 1.5 percent of the total resident population, while working-age migration has increased by a slightly larger percentage. In the four years 2014-2017, working age population increased by over 5.5 percent from net migration alone.

\subsubsection{Data}

We use national accounts data, migration data, house price data, and a tradeweighted aggregate of world gross domestic product (GDP) to estimate the model. The national account and migration data are sourced from Statistics New Zealand, while the house price data are from Quotable Value New Zealand. The trade-weighted world GDP data are compiled by the Reserve Bank of New Zealand. The data sample runs from 1992Q1-2017Q2.

GDP, residential investment, gross fixed capital formation (investment), and private consumption are sourced from the national accounts. We also use a trade-weighted measure of world GDP and working age net migration. All series are seasonally adjusted. The national accounts and migration data are transformed into per capita terms by dividing by seasonally-adjusted working age (15-65 year old) population. We take the natural logarithm of trending series and then apply the local linear projections of Hamilton

\footnotetext{
${ }^{12}$ These numbers are based on the destination country and country of origin, rather than country of citizenship.
} 
Figure 3.1: Net working-age migration flows into and out of New Zealand

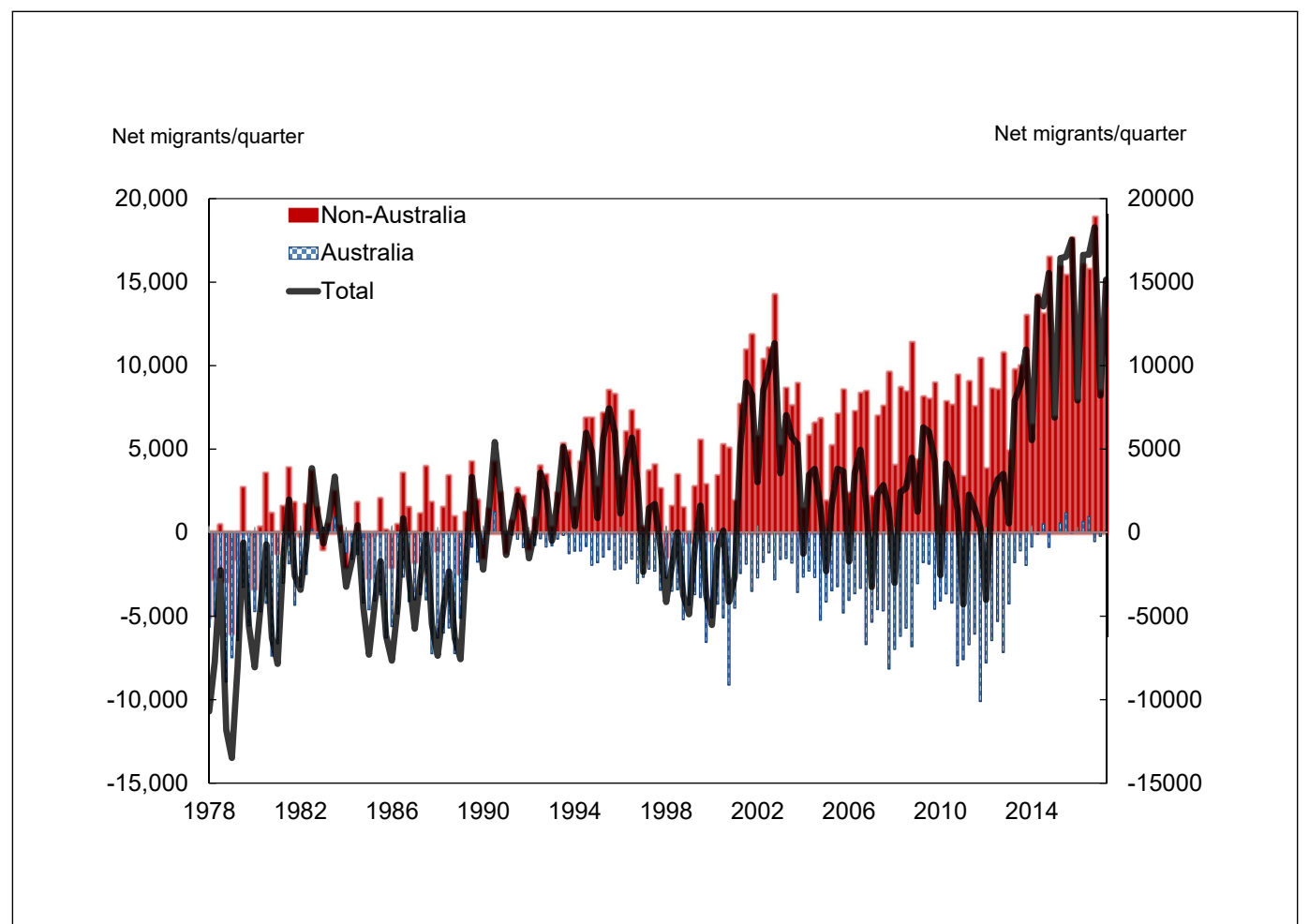

Note: The solid (black) line denotes non-seasonally adjusted working-age net migration into New Zealand. This figure is split into net migration into New Zealand from Australia (chequered blue bars; predominantly an out-flow) and net migration into New Zealand from all countries other than Australia (solid red bars). 
(2017) to compute the trends, and hence cycles, of the data series. ${ }^{13}$ This de-trending method is particularly straightforward to implement and consists of regressing the representative data series $x_{t+h}$ against a constant and the data $x_{t}, x_{t-1}, x_{t-2}$, and $x_{t-3}$, where $h=8$ quarters. This filter is onesided and thus avoids the so-called 'end-point' problem commonly associated with the Hodrick-Prescott filter. The filter has the added advantage that, given that four lags are used, it simultaneously strips out seasonality. Cycles derived from seasonally adjusted data and unadjusted data are virtually indistinguishable. Furthermore, the detrended series also has a mean of zero provided that a constant is included in the local linear projection. (See Hamilton 2017 for a thorough discussion of the virtues of this detrending method.) As the filter is not yet widely used, and as our migration data are not well-known, we illustrate the detrended data in figure 3.2. As can be see in the figure, the cycles obtained from a seasonally adjusted series and from an unadjusted migration series are virtually identical.

Over the course of our sample working age population has increased rapidly, from around 2.66 million people in 1992 to 3.84 million in 2017Q2, making it difficult to translate percentage changes into the number of migrants entering the country. The largest quarterly migrant impulse in the raw data in percentage terms corresponded to an increase in working age population of 0.4 percent in a single quarter, in 2015Q4. In this quarter (in unofficially seasonally adjusted terms) a little more than 15, 100 working age PLT migrants entered the country in raw terms.

The standard deviation of the detrended migration series is 0.00125 in quarter-on-quarter percent terms. Thus, in an ordinary year a one standard deviation increase in population from migration corresponds to roughly a $\frac{1}{2}$ percent of working age population. The largest detrended seasonally adjusted migration inflow in a quarter, 0.0031 percent, occurred in 2003Q1, and was nearly $2 \frac{1}{2}$ times as large as the standard deviation of the detrended series. Approximately 10,500 working age migrants entered New Zealand in that particular quarter in the raw data.

The rest-of-world gross domestic product series is a trade-weighted average of the GDPs from 17 countries. We have backcast the series 2 quar-

\footnotetext{
${ }^{13}$ The migration series is not logged as it takes both positive and negative values.
} 
Figure 3.2: Net working-age migration flows detrended via local linear projections

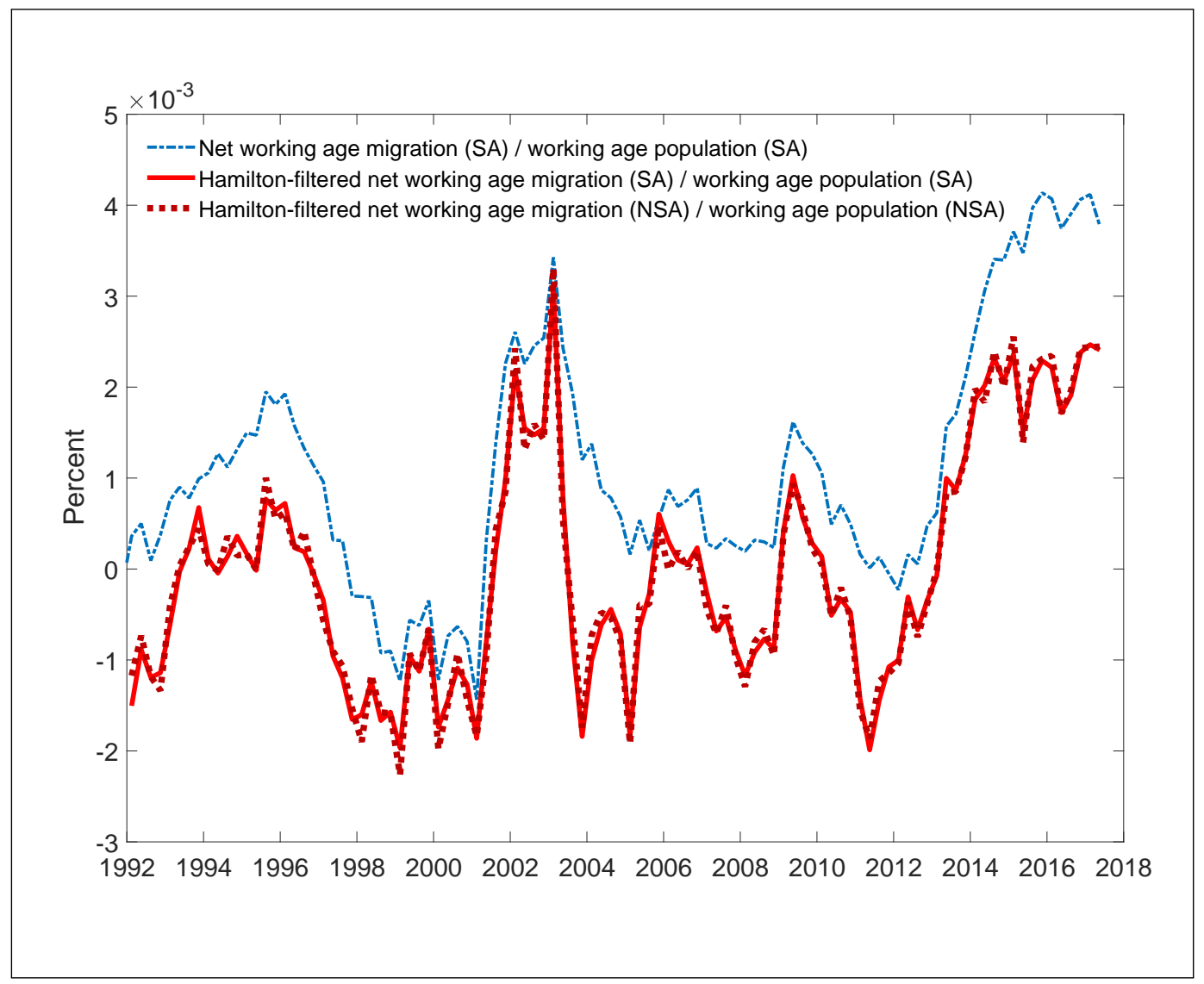

Note: The dash-dot (blue) line is the net migration working age impulse relative to the size of total working age population on a quarterly basis, seasonally adjusted but not de-trended. The solid and dotted (red) lines are the cycles derived from applying the Hamilton local linear projection to the dash-dot series and to an equivalent series that has not been seasonally adjusted. These cyclically adjusted series have means of zero by construction. The latter two lines illustrate that the Hamilton local projection can be used to eliminate both trends and seasonality simultaneously. 
Table 3.2: Raw data

\begin{tabular}{lll} 
Symbol & Description & RBNZ identifiers \\
\hline$G D P$ & Production GDP seasonally adjusted & ngdpp__z \\
$I^{\text {res }}$ & Private residential investment seasonally adjusted & nipd_z \\
$X$ & Gross fixed capita formation seasonally adjusted & ni_z \\
$C$ & Private consumption seasonally adjusted & ncp_z \\
pop wa & Working age (15-65 year old) population & lhpwa_z \\
$M$ & Net perm./long-term migration 15-65 year old & $-^{\dagger}$ \\
$q^{h}$ & Quotable Value House price index & pqhpi \\
$C P I$ & Consumer price index & pcpis ${ }^{\ddagger}$ \\
$G D P^{*}$ & Trade-weighted rest-of-world GDP & IWGDP_Z
\end{tabular}

${ }^{\dagger}$ Arrivals less departures. $\ddagger$ The CPI series used here slightly deviates from headline CPI in the early 1990s, as it excludes interest charges, which were incorporated in headline CPI at that time. The data are available from the authors upon request.

ters using an earlier vintage of this trade-weighted GDP, based on slightly fewer countries. Working age net migration is computed from Statistics New Zealand's permanent and long-term arrivals and departures data. The working-age data are assembled from age cohort data. We seasonally adjust the per capita working-age net migration data using a default implementation of X12. ${ }^{14}$ Table 3.2 defines the raw data, while table 3.3 describes the transformations applied to the raw data.

Table 3.4 reports the standard deviations, the standard deviation of variable $i$ relative to that of GDP and first-order autocorrelation to the observables. New Zealand GDP per capita is considerably more volatile than our measure of World GDP. Residential investment is 5.67 times as volatile as GDP and more volatile than gross fixed capital formation (investment). Real house prices are 3.8 times as volatile as GDP. Unlike most other developed economies, New Zealand consumption is somewhat more volatile than GDP. Net migration per capita in New Zealand is volatile by OECD standards, but is still only about 5 percent as volatile as real GDP.

\footnotetext{
${ }^{14}$ The executable file for X12 is available from the United States Census Bureau. We use an X12 implementation embedded in IRIS, https://github.com/IRIS-Solutions-Team.
} 
Table 3.3: Data transformations

\begin{tabular}{ll} 
Description & Symbol \\
\hline Log per capita income & $y=H A M\left(\log \left(G D P / p o p^{W A}\right)\right)$ \\
Log per capita residential investment & $H=H A M\left(\log \left(I^{\text {res }} / p o p^{W A}\right)\right)$ \\
Log per capita gross fixed capital formation & $x=H A M\left(\log \left(X / p o p^{W A}\right)\right)$ \\
Log per capita private consumption & $c=H A M\left(\log \left(C / p o p^{W A}\right)\right)$ \\
Log real house prices & $q^{h}=H A M\left(\log \left(P^{h} \cdot 1000 / C P I\right)\right)$ \\
Detrended migration per capita & $v=H A M\left(M / p o p^{W A}\right)$ \\
Log trade-weighted foreign GDP & $y^{*}=H A M\left(\log \left(G D P^{*}\right)\right)$ \\
\hline$H A M()$ represents the Hamilton filter used to detrend all series. $\log ()$ is the natural \\
logarithm. Migration is not logged because it can assume negative values. A Matlab file \\
implementing these transformations is available upon request.
\end{tabular}

Table 3.4: Observables and model moments

\begin{tabular}{lccc} 
& Std Dev $(\sigma)$ & $\sigma_{i} / \sigma_{y}$ & $\operatorname{Corr}\left(Y_{t}, Y_{t-1}\right)$ \\
\hline GDP per capita & 0.0264 & 1 & 0.8453 \\
Residential Investment per capita & 0.1496 & 5.67 & 0.8694 \\
Investment per capita & 0.1134 & 4.30 & 0.8297 \\
Consumption per capita & 0.0275 & 1.04 & 0.8408 \\
Real House Prices & 0.1006 & 3.81 & 0.8715 \\
World GDP & 0.0164 & 0.62 & 0.8864 \\
Migration per capita & 0.0013 & 0.05 & 0.8904 \\
\hline
\end{tabular}

Note: All data, except for net migration per capita, are in logs and and all are de-trended using the Hamilton filter. 


\subsubsection{Calibration and priors}

Columns 3 - 5 of Table 3.5 report the priors, means and the standard deviations of the parameters to be estimated. Most of our priors are fairly standard, see for instance Kamber et al. (2015). We do however, differ from the literature along several dimensions. Specifically, we attach a very tight prior to the share of capital, $\alpha$, with a prior mean of 0.33 , as is standard in the real business cycle literature. Likewise, the $\mathrm{AR}(1)$ coefficients for world GDP and net migration, $\rho_{y *}$ and $\rho_{v}$ respectively, have a prior that corresponds to estimates of these coefficients from single equation methods, as do the standard errors of the these two shocks, $\epsilon_{y *}$ and $\epsilon_{v}$. In each case, we estimate the parameter, but choose a relatively small standard error for our prior. These tight priors are implemented to prevent biases in the domestic equations from contaminating our estimates of these foreign impulses via the systems estimation of the model.

Preliminary efforts to estimate the ratio of human capital for migrants relative to domestic residents, $\chi$ proved problematic, so we calibrate this parameter and later report a sensitivity analysis in section 3.6 to illustrate how the dynamics of the model are affected by this parameter. The bottom half of Table 3.5 reports the calibrated parameters. Most of these are standard and only two parameters merit a special mention: the ratio of residential investment to consumption, which we set at 0.12 to match New Zealand data, and the above mentioned parameter $\chi$, which we set at 1.85 . The latter value is the relative level of human capital of migrants into New Zealand as reported in Boubtane et al. (2016).

\subsubsection{Estimation results}

Columns 6 - 8 of Table 3.5 report the posterior mean and lower and upper limits of 90 percent Bayesian confidence intervals from the posterior distribution. The share of capital in the production of goods has a posterior mean of 0.33 and the share of land in the housing sector has a posterior mean of 0.61. Capital depreciates 2.7 percent per quarter. The inverse of the labour supply elasticity, $\eta$, has a posterior mean of 3.7. The trade elasticity, the intra-temporal elasticity of substitution between home and foreign produced 
goods, is estimated to be 2.55, which implies that home and foreign-produced goods are highly substitutable for one another. The openness parameter, $\gamma$, also has a tight prior informed by the ratios of exports and imports to GDP. For New Zealand, this value is around 0.33. Parameters $a c u$ and $a c$ are the capacity utilisation elasticity and the investment adjustment cost parameter, respectively. $\phi^{b}$ measures the bond holding costs. The data suggests a low mean value 0.2 of one percent.

Total factor productivity (TFP) in goods production and housing is persistent, with estimated $\operatorname{AR}(1)$ coefficients of 0.76 and 0.72 , respectively. The corresponding standard deviations of the innovations are 0.03 and 0.04 , respectively. The shocks to preferences for housing and consumption have $\mathrm{AR}(1)$ coefficients of 0.86 and 0.83 , respectively. Whereas these two shocks have a similar persistence, the housing preference shock is more volatile than the consumption shock. The investment specific technology shock has a low autocorrelation coefficient and large standard deviation. The magnitude of this shock process is similar to estimates from Kamber et al. (2015). Working age migration per capita, estimated with a tight prior, is persistent with an associated $\mathrm{AR}(1)$ coefficient of 0.89 and a standard deviation of the migration impulse of 0.001 .

Trace-plot diagnostics of deciles are presented in appendix 3.B illustrating the convergence of the Markov chains that are used to sample from the posterior distribution. See Robert and Casella (2004) for a textbook treatment of the Monte Carlo methods used to estimate Bayesian models. 
Table 3.5: Estimated parameters values: $\chi=1.85$

\begin{tabular}{|c|c|c|c|c|c|c|c|}
\hline \multirow[b]{2}{*}{ Symbol } & \multirow[b]{2}{*}{ Description } & \multicolumn{3}{|c|}{ Prior } & \multicolumn{3}{|c|}{ Posterior } \\
\hline & & & Mean & Std Dev & Mean & $(5 \%$ & $95 \%)$ \\
\hline $\bar{\alpha}$ & Share of capital & $N$ & 0.330 & 0.010 & 0.330 & 0.314 & 0.346 \\
\hline$\xi_{h}$ & Share of land in housing & $N$ & 0.700 & 0.050 & 0.614 & 0.561 & 0.667 \\
\hline$\delta$ & Depreciation rate capital & $N$ & 2.500 & 0.500 & 2.748 & 1.944 & 3.538 \\
\hline$\eta$ & Frisch elasticity & $\Gamma$ & 2.000 & 0.750 & 3.733 & 2.211 & 5.251 \\
\hline$\theta$ & Intratemp. subst. elasticity & $N$ & 1.000 & 0.250 & 2.550 & 2.498 & 2.590 \\
\hline$\gamma$ & Openness & $\beta$ & 0.300 & 0.010 & 0.337 & 0.321 & 0.353 \\
\hline acu & Capacity-U curvature & $\beta$ & 0.500 & 0.150 & 0.669 & 0.479 & 0.865 \\
\hline$a c$ & Investment adjustment costs & $N$ & 4.000 & 1.500 & 6.313 & 4.433 & 8.131 \\
\hline $100 \cdot \phi^{b}$ & Bond adjustment costs & $\Gamma^{-1}$ & 1.000 & 5.000 & 0.205 & 0.152 & 0.256 \\
\hline$\rho_{a}$ & Persistence tech. & $\beta$ & 0.500 & 0.200 & 0.762 & 0.710 & 0.814 \\
\hline$\rho_{a h}$ & Persistence housing tech. & $\beta$ & 0.500 & 0.200 & 0.718 & 0.613 & 0.826 \\
\hline$\rho_{y}$ & Persistence foreign demand. & $\beta$ & 0.886 & 0.010 & 0.887 & 0.871 & 0.903 \\
\hline$\rho_{j}$ & Persistence housing pref. & $\beta$ & 0.500 & 0.200 & 0.860 & 0.806 & 0.917 \\
\hline$\rho_{j c}$ & Persistence consumption pref. & $\beta$ & 0.500 & 0.200 & 0.830 & 0.780 & 0.879 \\
\hline$\rho_{i}$ & Persistence investment-specific & $\beta$ & 0.500 & 0.150 & 0.272 & 0.145 & 0.397 \\
\hline$\rho_{v}$ & Persistence migration & $\beta$ & 0.890 & 0.010 & 0.890 & 0.874 & 0.906 \\
\hline$\sigma_{\epsilon_{a}}$ & Std dev. tech. & $\Gamma^{-1}$ & 0.004 & 1.500 & 0.030 & 0.026 & 0.034 \\
\hline$\sigma_{\epsilon_{h}}$ & Std dev. housing tech. & $\Gamma^{-1}$ & 0.005 & 1.500 & 0.038 & 0.032 & 0.043 \\
\hline$\sigma_{\epsilon_{y *}}$ & Std dev. foreign demand & $\Gamma^{-1}$ & 0.007 & 1.500 & 0.007 & 0.006 & 0.008 \\
\hline$\sigma_{\epsilon_{j}}$ & Std dev. housing pref. & $\Gamma^{-1}$ & 0.005 & 0.500 & 0.535 & 0.335 & 0.728 \\
\hline$\sigma_{\epsilon_{i}}$ & Std dev. investment-specific & $\Gamma^{-1}$ & 0.005 & 1.500 & 0.366 & 0.244 & 0.483 \\
\hline$\sigma_{\epsilon_{j c}}$ & Std dev. consumption pref. & $\Gamma^{-1}$ & 0.004 & 1.500 & 0.034 & 0.030 & 0.039 \\
\hline$\sigma_{\epsilon_{v}}$ & Std dev. migration & $\Gamma^{-1}$ & 0.001 & 1.500 & 0.001 & 0.001 & 0.001 \\
\hline & Calibrated & & & & & & \\
\hline$\chi$ & Relative human cap of migrants & & 1.85 & & & & \\
\hline $100 \cdot \delta_{h}$ & Depreciation rate housing & & 1 & & & & \\
\hline$\beta$ & Discount rate & & $1 / 1.01$ & & & & \\
\hline$\delta_{d}$ & Depreciation rate human cap. & & 0.01 & & & & \\
\hline$\phi_{s}$ & Skill accumulation & & 0.5 & & & & \\
\hline $\bar{j}$ & Steady-state j & & 0.7 & & & & \\
\hline$n+s$ & Hours worked + training & & $1 / 3$ & & & & \\
\hline$\xi_{m}$ & Share of traded goods in housing & & 0.1 & & & & \\
\hline$H / c$ & $\mathrm{H}$ - C ratio & & 0.12 & & & & \\
\hline
\end{tabular}




\subsection{A migration shock}

Migration increases a country's population and its labour supply. As a result, a positive migration shock, initially at least, reduces the per capita value of stocks such as capital, housing and bond holdings. As our calibration assumes that migrants have a higher stock of human capital than locals, the per capita stock of human capital rises in response to a migration shock. Much of the transitional dynamics of the model economy are therefore driven by the reversion of these stocks to their steady-state values following a shock to migration.

Another key driver of the model's dynamics following a migration shock is the response of the real exchange rate or (near-synonymously) the terms of trade. Figures 3.3-3.4, which show the impulse responses of the model using the mean of the estimated parameters, suggest that the terms of trade, defined as the price of foreign to home-produced goods, appreciate following an unexpected increase in migration. The reason the terms of trade appreciate is that a migration shock raises absorption of home-produced goods. The estimation results suggest that agents have a significant degree of home-bias in both consumption and investment expenditure (the smaller is the openness parameter $\gamma$, the greater is home bias), which raises demand for domestically produced goods by more than the demand for imports, and hence leads to an appreciation of the terms of trade. An appreciation of the terms of trade raises the return to domestic factors of production and increases the purchasing power of domestic consumers. The real appreciation, caused by the positive migration shock, thus has a positive wealth effect on consumption.

An increase in migration lowers the per capita physical capital stock. This reduction in capital per capita, along with the appreciation in the terms of trade, has the effect of raising the marginal product of capital. Thus owners of installed capital unambiguously benefit from an increase in migration. The increased return on capital stock raises investment. At the same time, an increase in migration raises the utilisation rate of capital. As Brunow et al. (2015, p. 1030) note, a constant returns to scale technology implies that per capita income growth rates decline when labour supply increases are not 
Figure 3.3: A migration shock (Panel A)

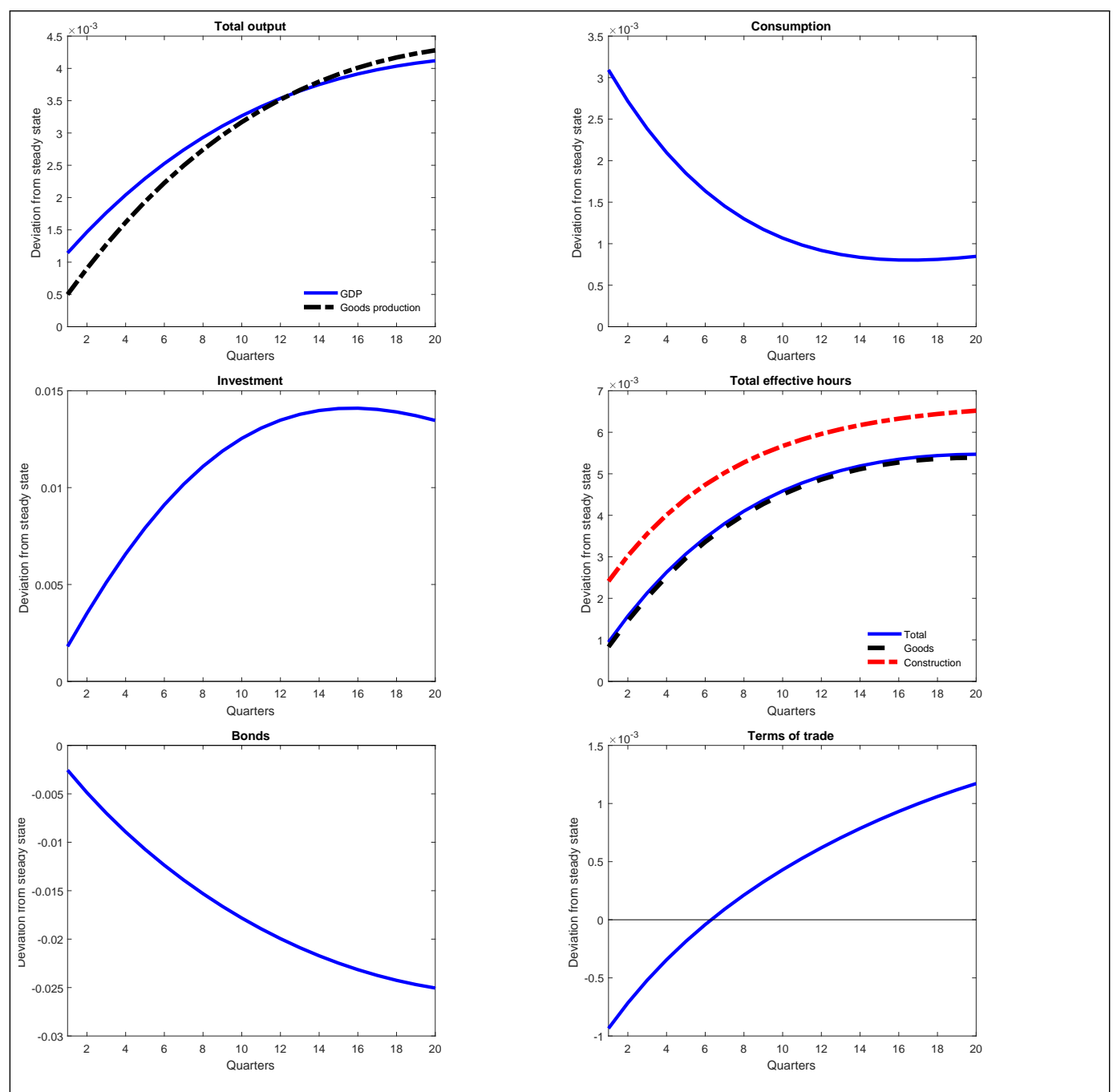

Note: An increase in migration in a small open economy. In the 'output' panel, the solid (blue) line denotes GDP, while the dash-dot (black) line denotes the home-produced traded goods. In the 'Total effective hours' panel, the solid thin (blue) line denotes total effective hours supplied by households, the dashed (black) line denotes effective hours devoted to goods production, while the dashed-dotted (red) line denotes effective hours in construction. 
Figure 3.4: A migration shock (Panel B)
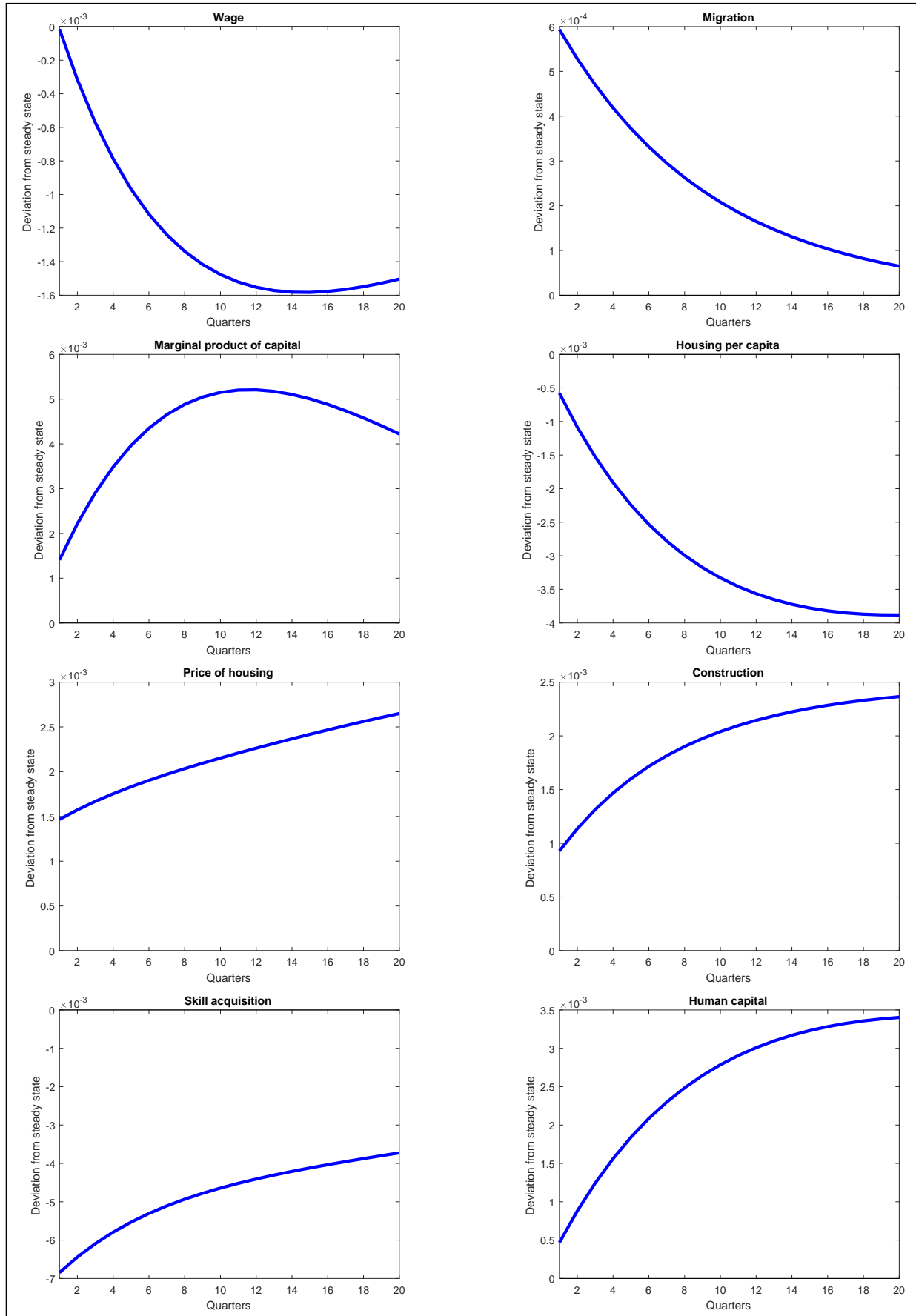

Note: An increase in migration in a small open economy. 
accompanied by corresponding increases in capital. In our model, however, changes in capacity utilisation partially offset the movements in capital per capita that arise with migration inflows and outflows.

Boubtane et al. (2016) estimate that the relative stock of human capital for migrants into New Zealand is 1.85 times that of the average domestic resident for the 1986-2006 period. Only the United States, with an estimated ratio of 0.97 , has a ratio below $1 .^{15}$ The remaining countries examined by Boubtane et al. have ratios from 1.01-2.87 (delimited by Greece and Ireland respectively). Using census data, Poot and Stillman (2016) find that migrants into New Zealand have 12.81 years of education on average, whereas New Zealand born individuals average a little less at 12.44 years of education. In 2006, 34 percent of migrants had bachelor degrees, while only 18 percent of New Zealand-born residents had such degrees. ${ }^{16}$ The mapping between different measures of schooling and human capital is difficult to resolve, so later we explore the sensitivity of our results to this key ratio.

As the empirical evidence suggests that migrants have a higher stock of human capital than New Zealand locals, we observe an increase in the per capita human capital stock following an increase in migration. As the transitional dynamics are characterised by a reversion to the pre-migration mean, the representative household reduces investment in skill acquisition. Less time spent training, means more time spent on hours worked. As a result, effective hours per capita increase following a rise in migration. The combination of a lower capital stock and an increased supply of effective labour, pushes down the wage rate. On impact, this effect is offset by the appreciation of the terms of trade. After a couple of quarters, the wage rate falls, before reverting back to the initial steady state in the medium run. The increase in effective hours plus the increase in capacity utilisation allow output per head to rise in response to a positive migration shock.

In the housing market, the per capita stock of housing is reduced by a sudden increase in migration. Given that migrants have the same preferences over housing and consumption as locals, the demand for new houses as well

\footnotetext{
${ }^{15}$ Borjas $(1994,1999 \mathrm{~b})$ confirms that the skill level of US immigrants is less than locals.

${ }^{16}$ See DOL (2009) and the 2006 New Zealand Census data, http://archive.stats. govt.nz/Census.aspx?.
} 
as the price of housing rises and the return on land increases. The increase in demand for new housing stock stimulates construction activity. Building houses requires land, labour and intermediate goods. Although total effective hours per worker increase, there is some reallocation of labour effort from the goods sector into the construction sector. Effective hours in the construction sector increase by more than in the goods producing sector. As noted earlier, we assume that the supply of building land is allowed to grow with the population to ensure that the post-migration steady-state is the same as the pre-migration steady state.

GDP is the sum of goods production and construction denoted by the solid (blue) line in the top left panel of figure 3.3. In the estimated model, goods production initially grows faster than overall GDP, though construction in the housing market overtakes around the three year mark. In summary, an increase in skilled migrants is expansionary for a small open economy. Even though the wage rate falls, per capita consumption, investment and GDP rises. Migration raises the return to stocks of physical capital and land and can temporarily reduce the return to human capital if migrants bring with them higher stocks of human capital. Our baseline business cycle results contrast with the cross-country panel data analysis of Brunow et al. (2015), who find that decadal averages of per capita GDP growth are unrelated to decadal movements in net migration rates. However, it should be noted that our conclusion is sensitive to the relative human capital of migrants to locals.

\subsubsection{Does migration drive the business cycle?}

Having analysed the dynamics of a migration shock in the model, we now consider whether migration is an important driver of the business cycle. Table 3.6 presents the variance decomposition at the posterior mean of our estimated model, with the lower and upper limit of the 90 percent Bayesian confidence intervals in square brackets underneath. Over our sample, the median contribution of the migration shock is 19 percent of the variance of observed GDP per capita. The rest of the variance is accounted for, in roughly equal parts, by the TFP shock and the preference for housing shock. 
Table 3.6: Variance decomposition at the posterior mean: $\chi=1.85$

\begin{tabular}{|c|c|c|c|c|c|c|c|}
\hline & & & Shocks & & & & \\
\hline Observables & $\epsilon_{a}$ & $\epsilon_{h}$ & $\epsilon_{y *}$ & $\epsilon_{j}$ & $\epsilon_{i}$ & $\epsilon_{j c}$ & $\epsilon_{v}$ \\
\hline$\overline{\text { GDP }}$ & $\begin{array}{c}0.36 \\
{[0.22,0.49]}\end{array}$ & $\begin{array}{c}0.04 \\
{[0.02,0.06]}\end{array}$ & $\begin{array}{c}0.00 \\
{[0.00,0.00]}\end{array}$ & $\begin{array}{c}0.35 \\
{[0.12,0.56]}\end{array}$ & $\begin{array}{c}0.04 \\
{[0.01,0.07]}\end{array}$ & $\begin{array}{c}0.02 \\
{[0.01,0.02]}\end{array}$ & $\begin{array}{c}0.19 \\
{[0.11,0.27]}\end{array}$ \\
\hline Investment & $\begin{array}{c}0.12 \\
{[0.05,0.18]}\end{array}$ & $\begin{array}{c}0.00 \\
{[0.00,0.00]}\end{array}$ & $\begin{array}{c}0.00 \\
{[0.00,0.00]}\end{array}$ & $\begin{array}{c}0.01 \\
{[0.00,0.01]}\end{array}$ & $\begin{array}{c}0.70 \\
{[0.55,0.85]}\end{array}$ & $\begin{array}{c}0.00 \\
{[0.00,0.00]}\end{array}$ & $\begin{array}{c}0.17 \\
{[0.07,0.27]}\end{array}$ \\
\hline $\begin{array}{l}\text { Residential } \\
\text { investment }\end{array}$ & $\begin{array}{c}0.00 \\
{[0.00,0.00]}\end{array}$ & $\begin{array}{c}0.46 \\
{[0.23,0.72]}\end{array}$ & $\begin{array}{c}0.00 \\
{[0.00,0.00]}\end{array}$ & $\begin{array}{c}0.50 \\
{[0.25,0.76]}\end{array}$ & $\begin{array}{c}0.00 \\
{[0.00,0.00]}\end{array}$ & $\begin{array}{c}0.01 \\
{[0.00,0.01]}\end{array}$ & $\begin{array}{c}0.03 \\
{[0.01,0.04]}\end{array}$ \\
\hline Consumption & $\begin{array}{c}0.24 \\
{[0.18,0.29]}\end{array}$ & $\begin{array}{c}0.00 \\
{[0.00,0.00]}\end{array}$ & $\begin{array}{c}0.00 \\
{[0.00,0.00]}\end{array}$ & $\begin{array}{c}0.02 \\
{[0.00,0.04]}\end{array}$ & $\begin{array}{c}0.07 \\
{[0.04,0.10]}\end{array}$ & $\begin{array}{c}0.56 \\
{[0.48,0.62]}\end{array}$ & $\begin{array}{c}0.12 \\
{[0.09,0.15]}\end{array}$ \\
\hline $\begin{array}{l}\text { Real house } \\
\text { prices }\end{array}$ & $\begin{array}{c}0.05 \\
{[0.01,0.08]}\end{array}$ & $\begin{array}{c}0.00 \\
{[0.00,0.01]}\end{array}$ & $\begin{array}{c}0.00 \\
{[0.00,0.00]}\end{array}$ & $\begin{array}{c}0.88 \\
{[0.79,0.98]}\end{array}$ & $\begin{array}{c}0.01 \\
{[0.00,0.02]}\end{array}$ & $\begin{array}{c}0.02 \\
{[0.00,0.03]}\end{array}$ & $\begin{array}{c}0.04 \\
{[0.01,0.07]}\end{array}$ \\
\hline
\end{tabular}

Recall that GDP consists of output of goods as well as housing. Migration is thus one of the main drivers of the variance of New Zealand GDP. For per capita consumption, migration is the third most important driver accounting for on average 12 percent of the variance, behind the consumption preference shock and the productivity shock. For investment, the migration shock accounts for on average 17 percent, which makes it the second most important driver behind the MEI (marginal efficiency of investment) shock. The role of migration shocks for the volatility of the housing market variables is modest, between 4 percent for real house prices and 3 percent for residential investment. The variance of residential investment is split relatively evenly between the housing sector productivity shock and the demand for housing shock, with a further 3 percent accounted for by migration. The variance of house prices is largely accounted for by the housing demand shock, which contributes 88 percent to the variability of real house prices. Migration accounts for 4 percent, which is more than is accounted for by the housing supply shock.

Given the relatively low degree of trade openness and the ability of the 
terms of trade to insulate the economy against foreign shocks, it is not surprising that the shock to world GDP has virtually no effect on the variances of our observables.

\subsubsection{Non-migration properties of the model}

The appendix to this chapter provides the impulse responses, historical shock decompositions, and the estimates of the (smoothed) shocks obtained from the Kalman filter. Section 3.C reports the IRFs, section 3.D illustrates the contributions of all shocks to the historical evolution of the observables, and 3.E depicts the seven primitive structural shocks that underpin these contributions.

The impulse responses for the non-migration shocks are broadly as one would expect. The generic technology shock induces co-movement in per capita GDP, consumption, investment, wages, residential investment and real house prices, with the terms of trade depreciating, reflecting the relative abundance of domestic goods.

A positive housing technology shock increases residential investment, and GDP more generally, but consumption and capital investment fall. Residential investment increases taking advantage of the positive technology shock for housing, but houses are now relatively cheaper to build so the price of houses falls. Labour supply is reallocated to build houses while residential investment is particularly productive, and wages increase, contributing another margin of labour adjustment. The terms of trade appreciate easing the decline in domestic goods production.

The foreign output shock in the model has a conventional effect, appreciating the terms of trade, increasing wages, but inducing substitution between housing and domestic goods. GDP and investment increase; an initial fall in consumption is offset by a longer-lived increase.

The preference shock for housing induces substitution between goods and housing. Increased demand for housing increases the relative price of houses and also stimulates residential investment. GDP increases but consumption of goods and capital investment falls. Like the housing technology shock, the terms of trade appreciates and wages increase. 
In this model, the investment-specific technology shock induces co-movement between GDP, consumption, house prices and capital investment. Residential investment dips on impact before recovering, with the terms of trade following a similar pattern. Wages, on the other hand are unambiguously above steady-state.

The consumption demand shock boosts output and consumption, but induces a relative shift away from housing: the relative price of housing falls as does residential investment. The terms of trade appreciates enabling substitution towards domestic goods. Capital investment declines on impact but eventually increases above steady-state. The increase in consumption outweighs the transitory decline in residential investment and GDP increases. However, wages are predominantly below steady-state.

The migration shock has been described in the main text, and is not addressed further here in this appendix.

The historical shock decompositions provide a sample-specific illustration of the variance decompositions reported in table 3.6. Per capita GDP, for example, is predominantly driven by the technology and housing preference shocks. Consumption is driven by the technology and consumption preference shocks, with the investment-specific technology shock making a small contribution too. Per capita investment is driven by this same investmentspecific technology shock, with a surprisingly large contribution coming from the standard technology shock and a small contribution from migration in this sample. The contribution of migration is particularly strong at the end of the sample, when migration is of course at an historic high. Residential investment per capita is driven by the housing demand technology shock and the housing preference shock, while the real house price is driven by the housing demand shock and technology.

Interestingly, only the migration shock causes a simultaneous increase in hours worked in both the housing and goods sectors. All other shocks induce movements in opposite directions. 


\subsection{Sensitivity analysis: The relative human capital of migrants}

One of the key assumptions of our DSGE model is that, on average, migrants have higher human capital levels than locals. As our data is not informative about this parameter, we calibrated this parameter $\chi$ to a baseline value of 1.85, which is the value estimated by Boubtane et al. (2016) for New Zealand for the period from 1986 to 2006 . We justified this claim by noting that skilled and entrepreneurial migrants are a large proportion of total migration into New Zealand. Here we re-estimate the model under the assumption that migrants' human capital stock does not differ from that of locals, and explore the contribution that migration shocks then make to the variability of our observables.

Tables 3.7 and 3.8 reports the parameter estimates and the variance decomposition for a model where $\chi$ has been set to 1 . Cancelling out the effects of migration on the stock of human capital does not significantly alter the model's parameter estimates, see table 3.7 , but does significantly reduce the contribution of the migration shock to the variance of our observables. For per capita GDP, the contribution falls from around 19 percent to 0.2 percent, for residential investment the figure drops from 3 percent to 0 percent. For consumption per capita the migration shock's contribution of the total variance falls from 12 percent to just 3 percent and for house prices from 4 percent down to 1 percent. Our results thus imply that migration has less of an effect on the business cycle when migrants are closer to the local population in terms of their human capital. Our business cycle results thus cohere with an observation by Dustmann et al. (2005, p. F324), namely that "labour market effects of immigration depend most importantly on the structure of the receiving economy, as well as the skill mix of immigrants, relative to the resident population." Figure 3.5 illustrates how the contribution of migration shocks varies with the parameter $\chi$. The relationship is U-shaped, with minima for $\chi \in(0.5,1)$, i.e., where migrants have lower or equivalent levels of human capital relative to locals. 
Table 3.7: Estimated parameter values: $\chi=1$

\begin{tabular}{|c|c|c|c|c|c|c|c|}
\hline \multirow[b]{2}{*}{ Symbol } & \multirow[b]{2}{*}{ Description } & \multicolumn{3}{|c|}{ Prior } & \multicolumn{3}{|c|}{ Posterior } \\
\hline & & & Mean & Std Dev & Mean & $(5 \%$ & $95 \%)$ \\
\hline$\alpha$ & Share of capital & $N$ & 0.330 & 0.010 & 0.330 & 0.313 & 0.346 \\
\hline$\alpha_{h}$ & Share of land in housing & $N$ & 0.700 & 0.050 & 0.612 & 0.560 & 0.665 \\
\hline $100 \cdot \delta$ & Depreciation rate capital & $N$ & 2.500 & 0.500 & 2.791 & 2.011 & 3.577 \\
\hline$\eta$ & Frisch elasticity & $\Gamma$ & 2.000 & 0.750 & 3.778 & 2.223 & 5.294 \\
\hline ces & Intratemp. subst. elasticity & $N$ & 1.000 & 0.250 & 2.551 & 2.501 & 2.590 \\
\hline$\gamma$ & Openness & $\beta$ & 0.300 & 0.010 & 0.338 & 0.322 & 0.354 \\
\hline acu & Capacity-U curvature & $\beta$ & 0.500 & 0.150 & 0.667 & 0.477 & 0.863 \\
\hline$a c$ & Investment adjustment costs & $N$ & 4.000 & 1.500 & 6.348 & 4.525 & 8.173 \\
\hline $100 \cdot \phi^{b}$ & Bond adjustment costs & $\Gamma^{-1}$ & 1.000 & 5.000 & 0.204 & 0.152 & 0.253 \\
\hline$\rho_{a}$ & Persistence tech. & $\beta$ & 0.500 & 0.200 & 0.756 & 0.703 & 0.808 \\
\hline$\rho_{a h}$ & Persistence housing tech. & $\beta$ & 0.500 & 0.200 & 0.714 & 0.609 & 0.823 \\
\hline$\rho_{y}$ & Persistence foreign demand. & $\beta$ & 0.886 & 0.010 & 0.887 & 0.871 & 0.903 \\
\hline$\rho_{j}$ & Persistence housing pref. & $\beta$ & 0.500 & 0.200 & 0.861 & 0.806 & 0.917 \\
\hline$\rho_{j c}$ & Persistence consumption pref. & $\beta$ & 0.500 & 0.200 & 0.833 & 0.785 & 0.881 \\
\hline$\rho_{i}$ & Persistence investment-specific & $\beta$ & 0.500 & 0.150 & 0.272 & 0.145 & 0.397 \\
\hline$\rho_{v}$ & Persistence migration & $\beta$ & 0.890 & 0.010 & 0.890 & 0.874 & 0.907 \\
\hline$\epsilon_{a}$ & Std dev. tech. & $\Gamma^{-1}$ & 0.004 & 1.500 & 0.030 & 0.026 & 0.034 \\
\hline$\epsilon_{h}$ & Std dev. housing tech. & $\Gamma^{-1}$ & 0.005 & 1.500 & 0.037 & 0.032 & 0.043 \\
\hline$\epsilon_{y *}$ & Std dev. foreign demand & $\Gamma^{-1}$ & 0.007 & 1.500 & 0.007 & 0.006 & 0.008 \\
\hline$\epsilon_{j}$ & Std dev. housing pref. & $\Gamma^{-1}$ & 0.005 & 0.500 & 0.533 & 0.337 & 0.728 \\
\hline$\epsilon_{i}$ & Std dev. investment-specific & $\Gamma^{-1}$ & 0.005 & 1.500 & 0.367 & 0.247 & 0.485 \\
\hline$\epsilon_{j c}$ & Std dev. consumption pref. & $\Gamma^{-1}$ & 0.004 & 1.500 & 0.034 & 0.030 & 0.039 \\
\hline$\epsilon_{v}$ & Std dev. migration & $\Gamma^{-1}$ & 0.001 & 1.500 & 0.001 & 0.001 & 0.001 \\
\hline & Calibrated & & & & & & \\
\hline$\chi$ & Relative human cap of migrants & & 1 & & & & \\
\hline $100 \cdot \delta_{h}$ & Depreciation rate housing & & 1 & & & & \\
\hline$\beta$ & Discount rate & & $1 / 1.01$ & & & & \\
\hline$\delta_{d}$ & Depreciation rate human cap. & & 0.01 & & & & \\
\hline$\phi_{s}$ & Skill accumulation & & 0.5 & & & & \\
\hline $\bar{j}$ & Steady-state j & & 0.7 & & & & \\
\hline$n+s$ & Hours worked + training & & $1 / 3$ & & & & \\
\hline$\xi_{m}$ & Share of traded goods in housing & & 0.1 & & & & \\
\hline$H / c$ & $\mathrm{H}-\mathrm{C}$ ratio & & 0.12 & & & & \\
\hline
\end{tabular}


Figure 3.5: The role of migration shocks as a function of $\chi$
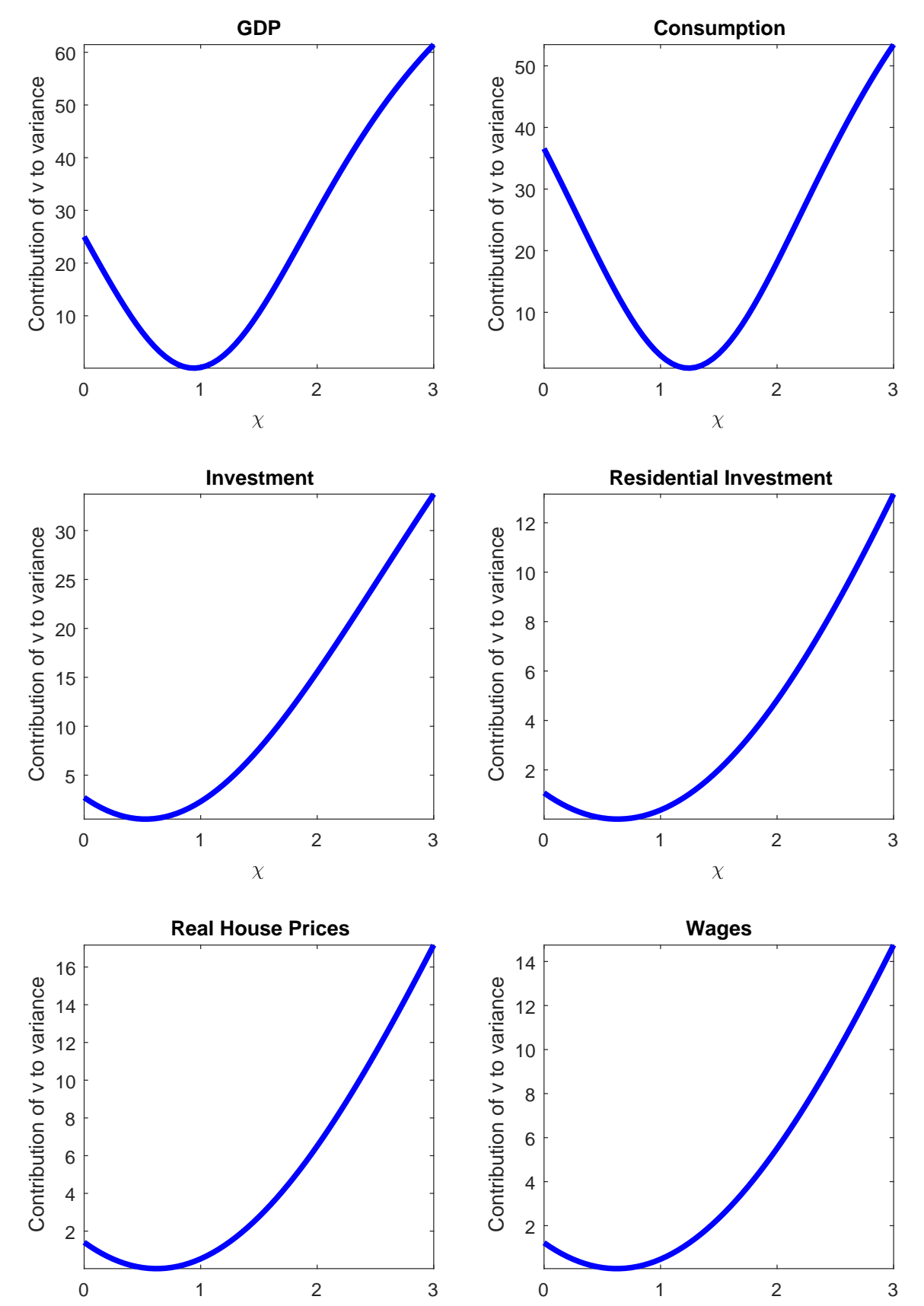

Note: This figure reports the variance contributions of the migration shock for different values of the relative human capital parameter $\chi$, migrant capital to local, with all other parameters held constant at the mean posterior values. 
Table 3.8: Variance decomposition at the posterior mean: $\chi=1$

\begin{tabular}{lccccccc} 
Observables & $\epsilon_{a}$ & $\epsilon_{h}$ & $\epsilon_{y *}$ & $\epsilon_{j}$ & $\epsilon_{i}$ & $\epsilon_{j c}$ & $\epsilon_{v}$ \\
\hline GDP & 0.46 & 0.06 & 0.00 & 0.35 & 0.10 & 0.03 & 0.00 \\
& {$[0.28,0.62]$} & {$[0.03,0.09]$} & {$[0.00,0.00]$} & {$[0.13,0.56]$} & {$[0.03,0.16]$} & {$[0.02,0.04]$} & {$[0.00,0.00]$} \\
Investment & 0.08 & 0.00 & 0.00 & 0.00 & 0.88 & 0.00 & 0.03 \\
& {$[0.03,0.14]$} & {$[0.00,0.00]$} & {$[0.00,0.00]$} & {$[0.00,0.01]$} & {$[0.81,0.95]$} & {$[0.00,0.00]$} & {$[0.01,0.05]$}
\end{tabular}

\subsection{An SVAR look at the data}

Having investigated the business cycle effects of migration via a DSGE model, we now look at the data using a structural vector autoregression (SVAR). As SVARs embody fewer restrictions, we do not necessarily expect to find the exact same dynamics as in the DSGE model. Instead, our focus is on the qualitative effects of a migration shock on the variables in our data set. In this section, we ask whether or not a migration shock in an SVAR is expansionary for the components of GDP, whether it raises residential investment and house prices, and whether it causes the real exchange rate to appreciate. In other words, are the qualitative dynamics of an SVAR comparable with those generated by the estimated DSGE model?

We develop an SVAR from the same observable variables that were used to estimate the DSGE model, but augmented with the real wage and the real exchange rate, both logged and detrended as previously described. We specify the VAR as follows

$$
A_{0} y_{t}=A(L) y_{t-1}+u_{t} .
$$


where $A_{0}$ is a $k+1 \times k+1$ matrix; $y_{t}$ is a $k+1$ column vector of variables, including a 1 to account for constants, and $A(L) \equiv A_{1} L+A_{2} L^{2}+\ldots A_{p} L^{p}$ denotes a lag polynomial where $L$ is the lag operator, such that $L y_{t}=y_{t-1}$. The vector $u_{t}$ represents the mean-zero, serially uncorrelated exogenous shocks with diagonal variance-covariance matrix $\Sigma_{u}$. The reduced form errors thus have a variance covariance matrix $A_{0}^{-1} \Sigma_{u}\left(A_{0}^{-1}\right)^{\prime}$.

Migration shocks are identified through a recursive identification scheme. We treat world GDP as an exogenous variable, ordered first in the causal ordering, followed by per capita migration. ${ }^{17}$ Migration shocks are assumed to be uncorrelated with contemporaneous shocks to domestic variables. While we employ a Cholesky decomposition to identify the world GDP and migration shocks, we disregard the exact ordering of the subsequent domestic shocks, since they are not of material importance to our migration analysis. The lack of contemporaneous correlation seems a reasonable identifying assumption given that obtaining a visa or going through the logistics of leaving a job and moving from one country to another can be a lengthy process.

We use a multivariate Bayesian information criterion (MBIC) to determine the lag structure of the model. Unsurprisingly, given the comparatively strong penalty on the number of parameters and hence the preference for parsimony, the MBIC implies that the reduced form VAR has a single lag. ${ }^{18}$

In our DSGE model we assume that the migration impulse is exogenous to the domestic economy. This assumption may seem a little implausible, since the propensity to migrate should reflect the relative costs and benefits in home and foreign countries (see for example Clark et al. 2007, Mayda 2010, and Hatton 1995). To explore whether domestic pull factors are material explanators of our migration series we conduct a simple Granger-causality test. We focus on the migration equation alone, and consider simple autoregressive processes against single equations that have lags of domestic variables as additional regressors.

When the migration equation from a $\operatorname{VAR}(1)$ is compared to an $\operatorname{AR}(1)$ process for migration, a likelihood ratio test cannot reject the restrictions

\footnotetext{
${ }^{17}$ The migration process is equivalent to what one would obtain from a VARX model with contemporaneous and lagged world GDP included as exogenous regressors.

${ }^{18}$ The Hannan-Quinn information criterion also implies a VAR model with one lag, while the Akaike information criterion implies that four lags should be included.
} 
embedded in the $\mathrm{AR}(1)$. The dynamics of the migration equation are predominantly affected by its own lags. This block exogeneity assumption is not material to our qualitative results. The impulse responses are virtually identical if one specifies the migration equation as an autoregressive process or as an unrestricted equation from a $\operatorname{VAR}(1)$, with feedback from lagged domestic variables. Specifying migration as an exogenous process does, however, have the benefit of making our SVAR model broadly comparable with the reduced form of the DSGE model. ${ }^{19}$

In Figure 3.6 we report the impulse response shock to migration. The independent and identically distributed migration shock has the same standard deviation as in the DSGE model (0.00059), which of course is propagated via the $\mathrm{AR}(1)$ process used to model the migration series. In the long-run this shock corresponds to a cumulative impulse of roughly 0.0053 percent of working age population. This is larger than the simple standard deviation of the working age migration series, but reflects the fact that migration impulses exhibit a strong degree of autocorrelation. The migration shock is associated with a statistically significant increase in consumption, investment, residential investment, and house prices. GDP per capita also increases, but is not significantly different from zero. The VAR impulse response also confirms one of the key transmission mechanisms of a migration shock, namely the appreciation of the real exchange rate associated with a migration shock.

\footnotetext{
${ }^{19}$ Given that the exogenous shocks in the DSGE model are AR(1) processes the reduced form of the DSGE model corresponds to a $\operatorname{VAR}(2)$.
} 
Figure 3.6: A migration shock in a VAR

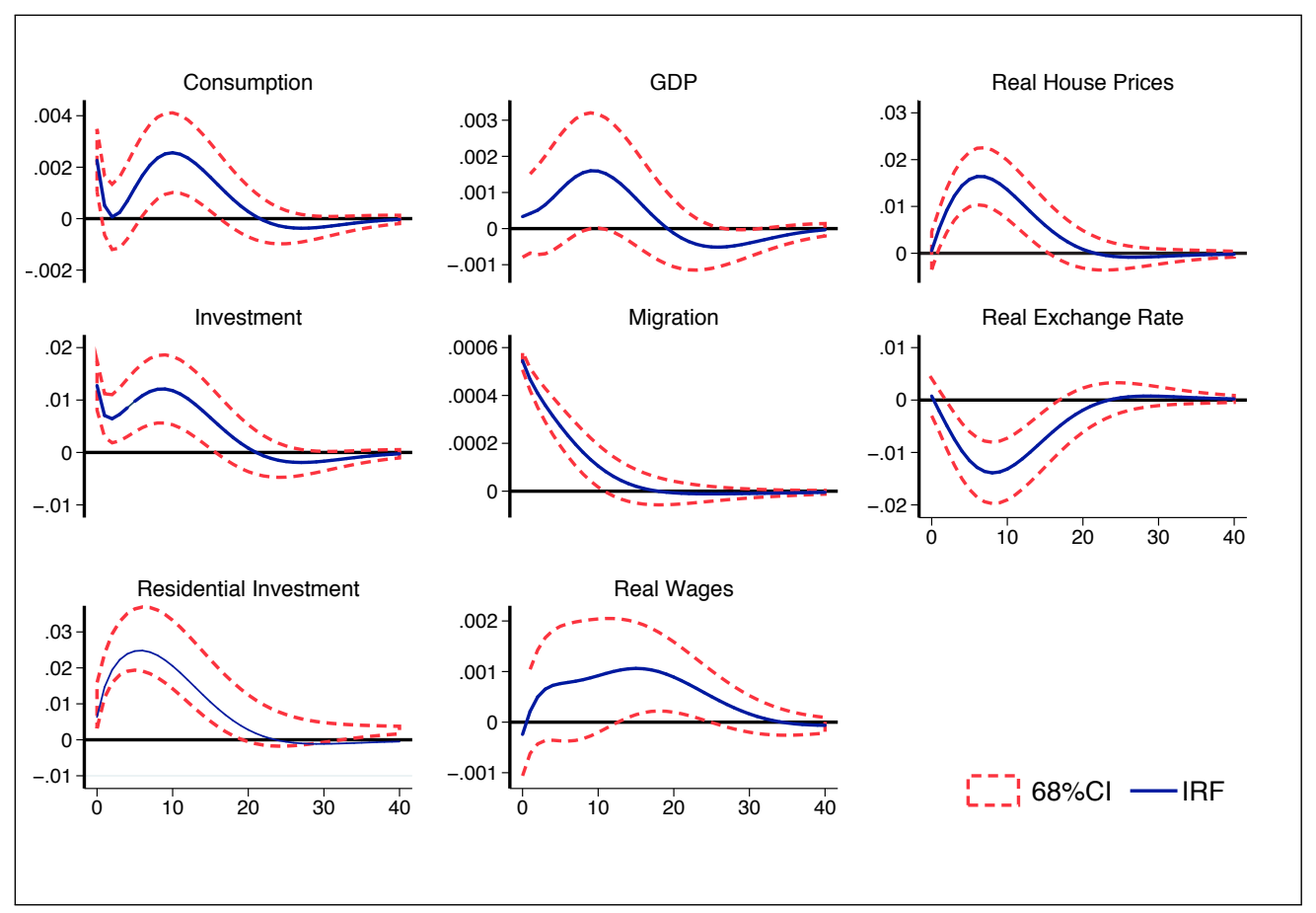

Notes: An increase in migration in a small open economy. Migration shock in the VAR(1) is identified by a Cholesky decomposition where the filtered world GDP series is ordered first followed by the migration per capita series which is ordered second. All data series are logged and de-trended using the Hamilton (2017) filter, as in the Bayesian estimation. The dashed lines are $68 \%$ confidence intervals. A decline in the real exchange rate series denotes a real appreciation. 


\subsection{Conclusion}

Migration shocks matter for the business cycle. Using a dynamic stochastic general equilibrium model of a small open economy estimated on data for New Zealand, we find that migration shocks account for a considerable proportion of the variability of per capita GDP. For the components of per capita GDP, migration shocks matter, but are not the key drivers. Even for residential investment and real house prices, migration shocks make some contribution, though other shocks - housing technology and housing preference shocks are far more important.

An unexpected positive migration shock is found to be expansionary in terms of per capita real GDP and its components and is associated with an initial appreciation of the terms of trade. As expected, migration benefits the owners of fixed assets such as capital or housing: the returns on these assets rise with an influx of migrants. The return on human capital is also affected by the relative human capital of migrants versus locals. If, as in our case, migrants have an initially higher level of human capital than locals, the real wage, or the return on effective labour falls.

The relative level of human capital of migrants also affects the extent to which migration shocks contribute to the volatility of per capita GDP. We conduct a sensitivity analysis on the relative level of human capital. We find that the impact of migration shocks for the business cycle is much diminished if new migrants and locals have similar levels of human capital. When we assume that migrants have the same level of human capital as locals, migration shocks make only a minor contribution to the variances of per capita GDP and other macro variables. 


\section{Appendix}

\section{A Steady state}

$$
\begin{aligned}
\mu & =1 / c \\
0 & =\phi_{0}(n+s)^{\eta}+(1 / c) w d \\
0 & =-\phi_{0}(n+s)^{\eta}+\lambda \phi_{s} \frac{(d s)^{\phi_{s}}}{s} \\
\lambda & =(1 / c) w n+\beta \lambda\left[\phi_{s} \frac{(s d)^{\phi_{s}}}{d}+\left(1-\delta_{d}\right)\right] \\
1 & =\frac{(d s)^{\phi_{s}}}{d}+\left(1-\delta_{d}\right) \\
1 & =\beta(1+r) \\
\frac{q^{H} h}{c} & =\frac{j}{\left(1-\beta\left(1-\delta_{h}\right)\right)} \\
p^{l} & =\beta\left(p^{l}+R^{l}\right)
\end{aligned}
$$




$$
\begin{aligned}
\frac{n}{s} & =\frac{\left(1-\beta\left(\delta_{d}\left(\phi_{s}-1\right)+1\right)\right.}{\phi_{s} \delta_{d}} \\
n+s & =1 / 3 \\
n & =\frac{(n+s) \frac{n}{s}}{\frac{n}{s}+1} \\
s & =(n+s)-s \\
\frac{y}{k} & =\frac{1 / \beta-(1-\delta)}{\alpha} \\
\frac{c}{k} & =\frac{y}{k}-\delta \\
\frac{k}{n} & =\left(\frac{y}{k}\right)^{1 /(\alpha-1)} \\
\frac{n^{y}}{n^{h}} & =\frac{y}{k} \frac{k}{c} \frac{1}{h} \frac{1}{\delta_{h} q^{H}} \\
\frac{n}{n^{h}} & =1+\frac{n^{y}}{n^{h}}
\end{aligned}
$$

\section{B MCMC convergence}

This appendix illustrates diagnostics to assess whether the Markov chains have converged to the posterior distributions of interest. Two Markov chains were iterated 2,000,000 times to estimate the posterior distribution of the vector of parameter. As noted by Brooks and Roberts (1998) and others, no diagnostic can guarantee convergence. Nevertheless, these figures provide some reassurance that the chains have indeed converged to their stationary distributions. The figures below illustrate the deciles for each chain, computed recursively as the chains are iterated forward (following a 100,000 burn-in period to reduce bias from initial conditions). Convergence implies that like-deciles from the two chain should asymptote to the same values, which should remain constant as the sample is extended. The variability evident in some deciles for some parameters seems fairly modest and is unlikely to be of economic significance. 
Figure 3.B.1: MCMC convergence - deciles (Panel A)

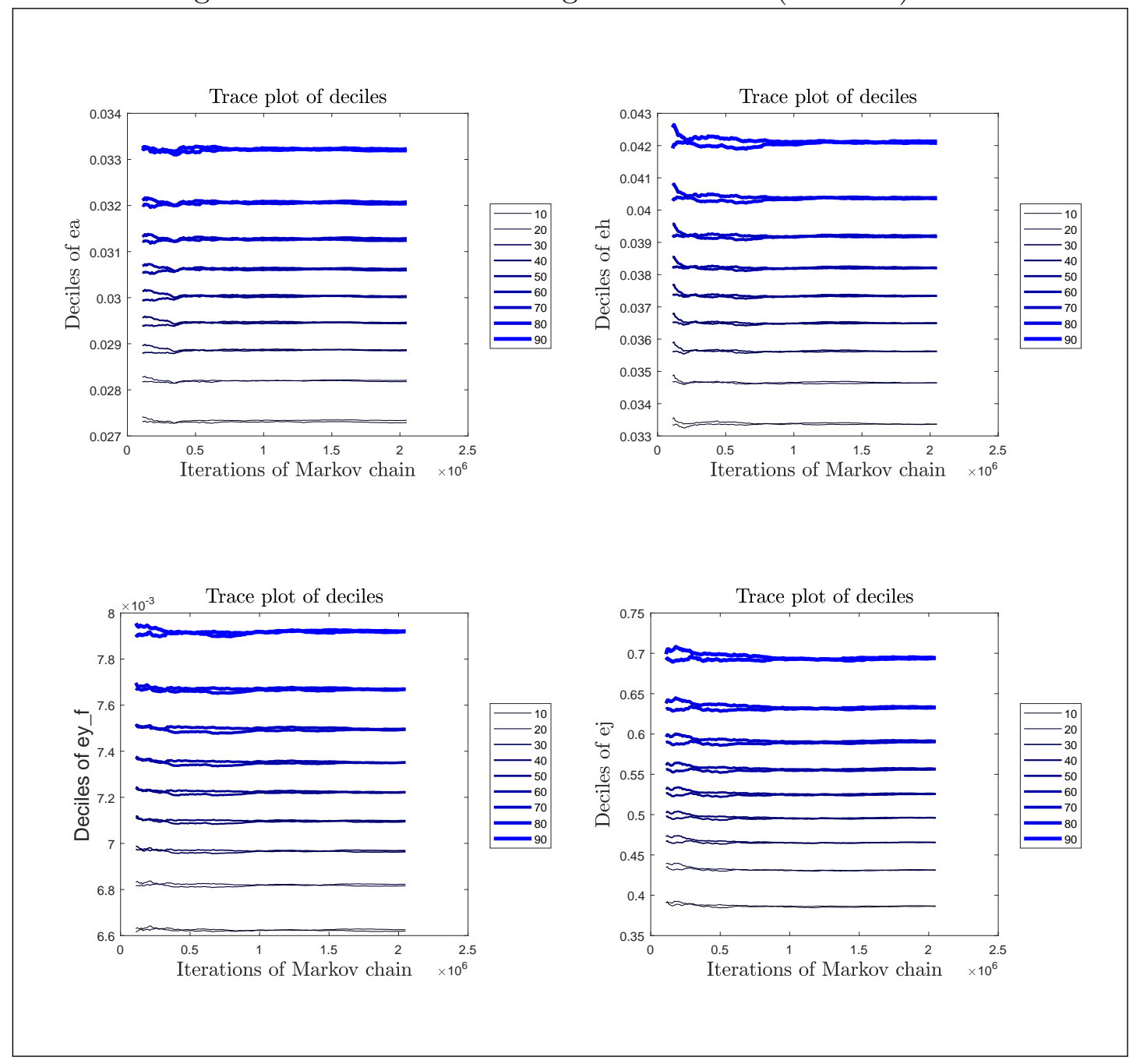


Figure 3.B.2: MCMC convergence - deciles (Panel B)

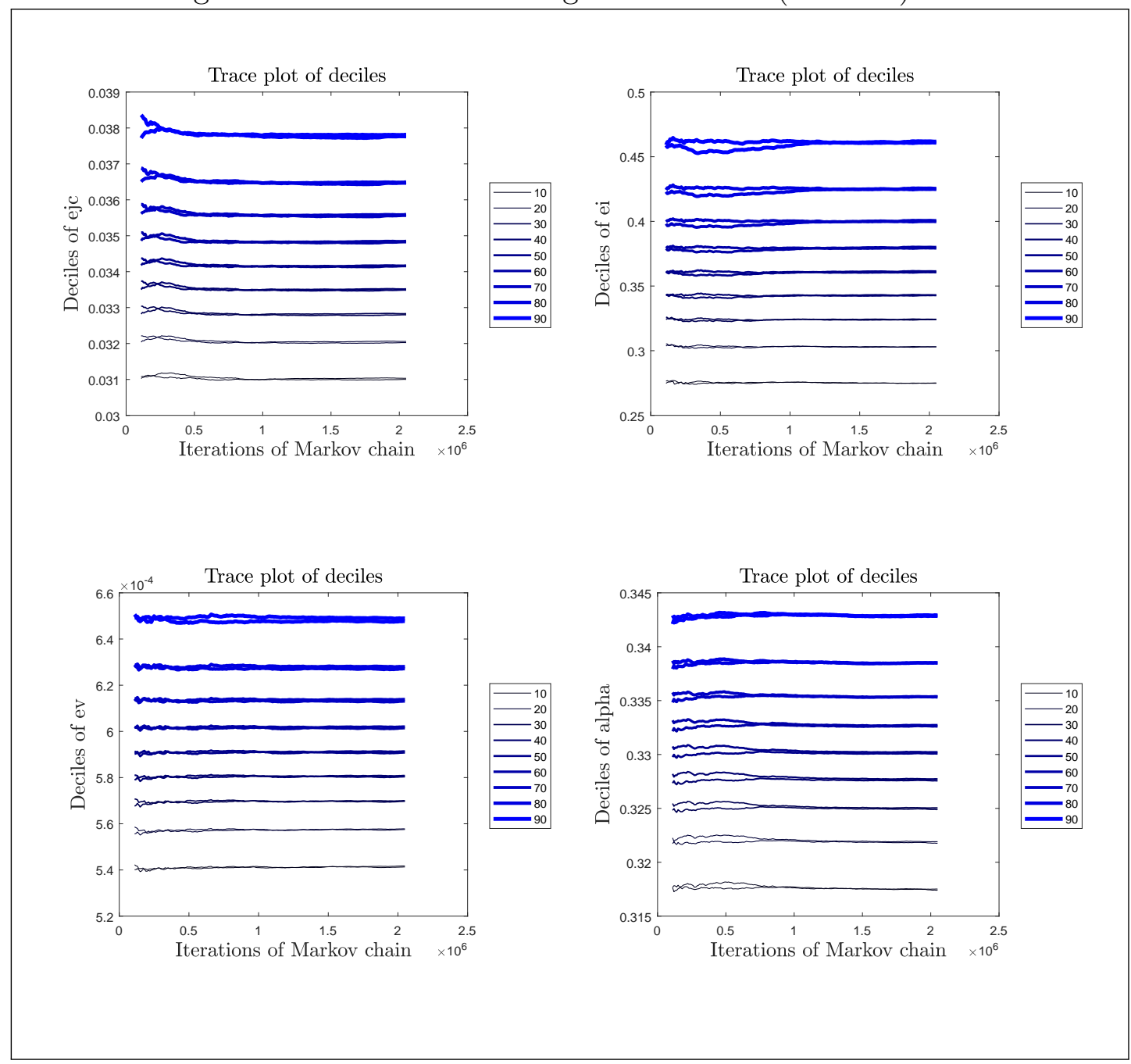


Figure 3.B.3: MCMC convergence - deciles (Panel C)

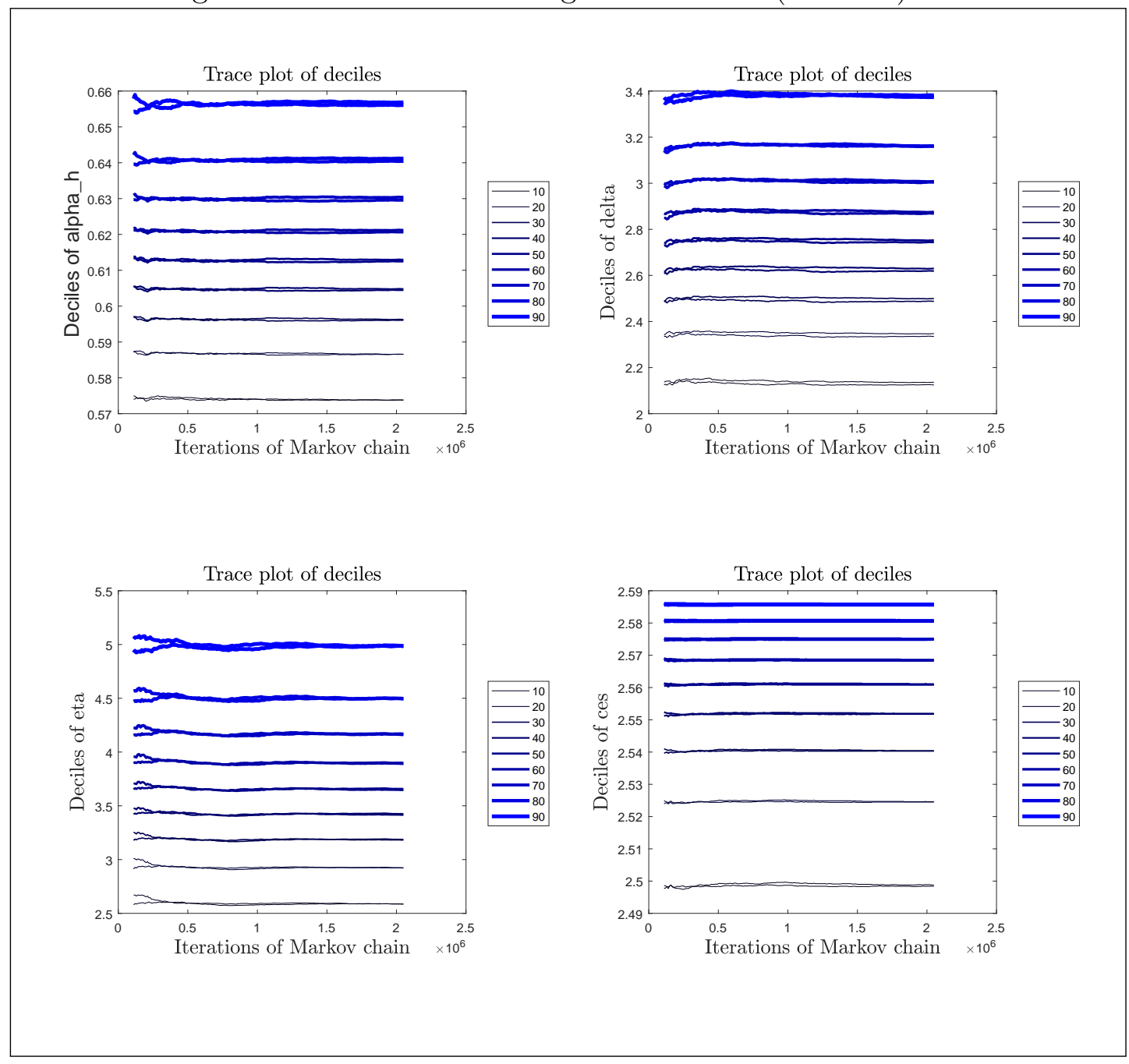


Figure 3.B.4: MCMC convergence - deciles (Panel D)

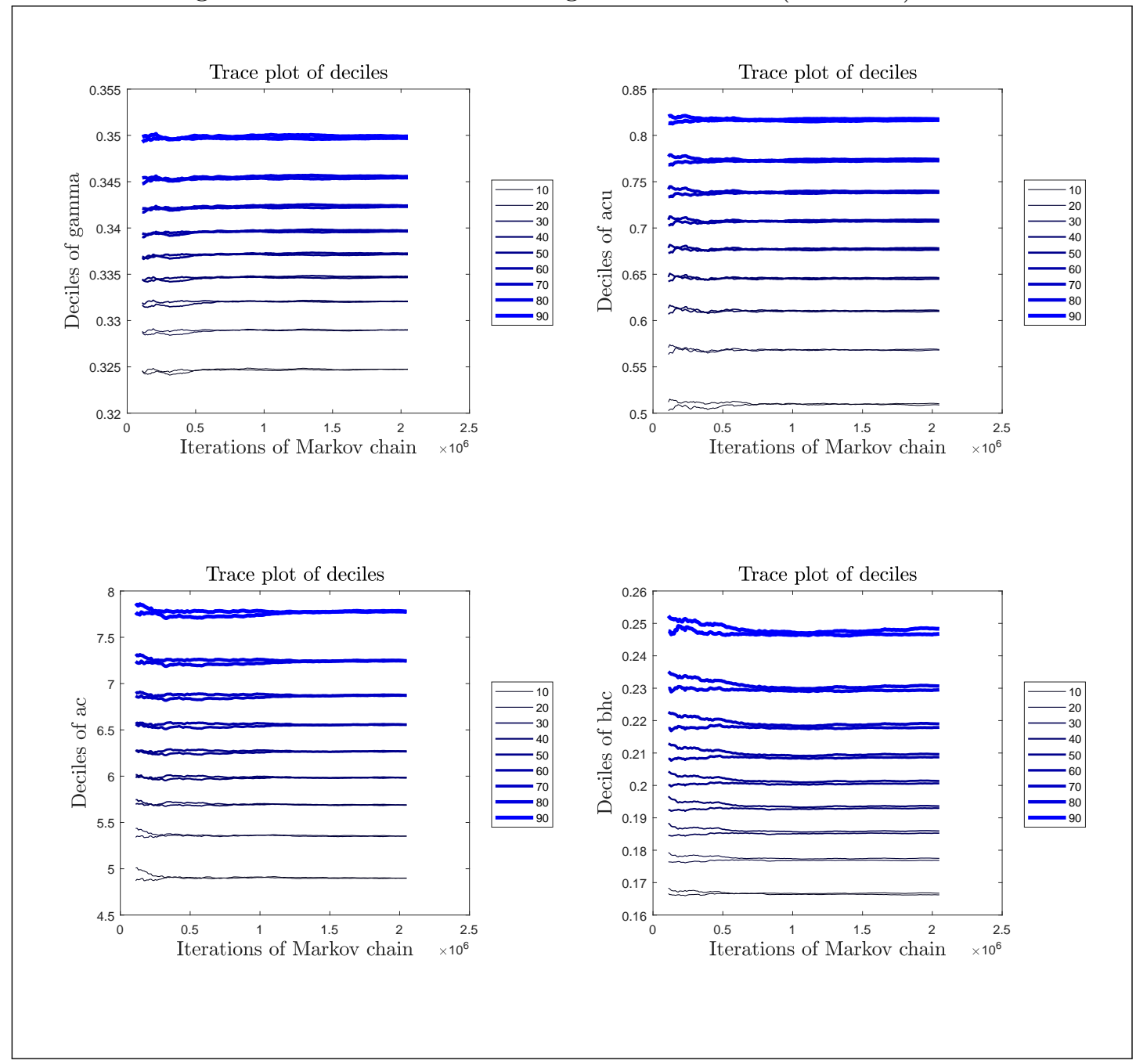


Figure 3.B.5: MCMC convergence - deciles (Panel E)

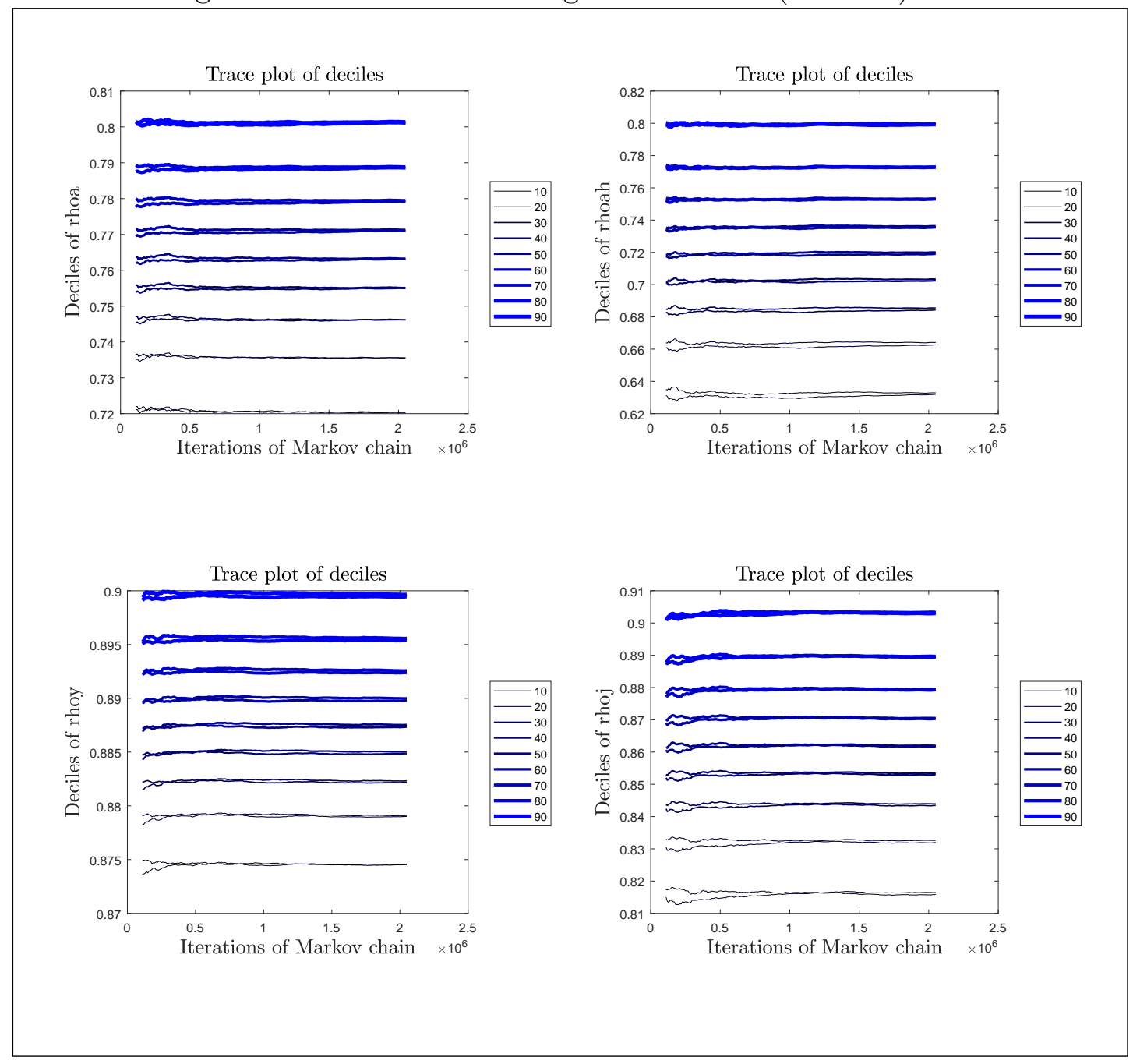


Figure 3.B.6: MCMC convergence - deciles (Panel F)

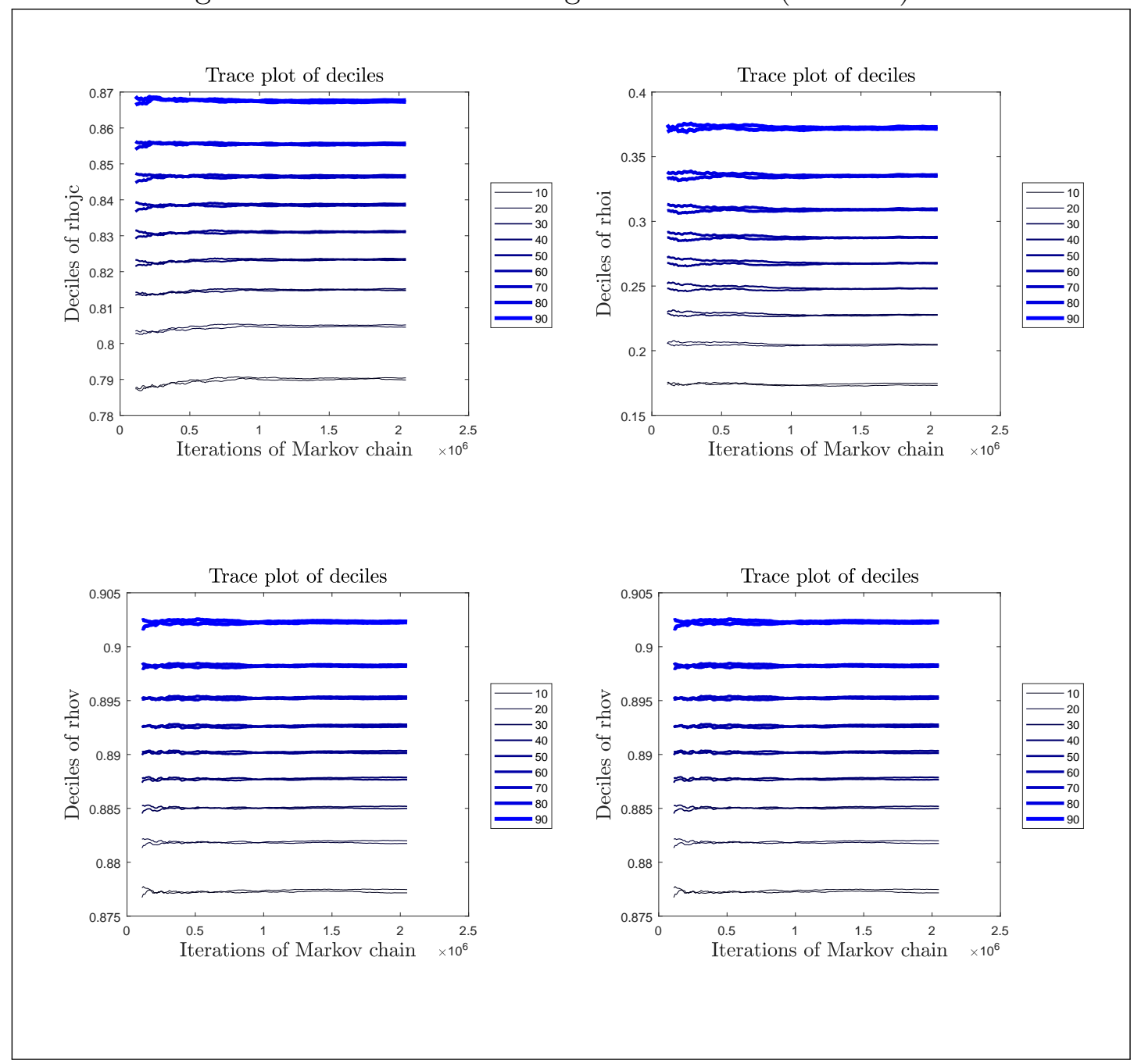




\section{C Impulse response functions}

Figure 3.C.1: Impulse responses

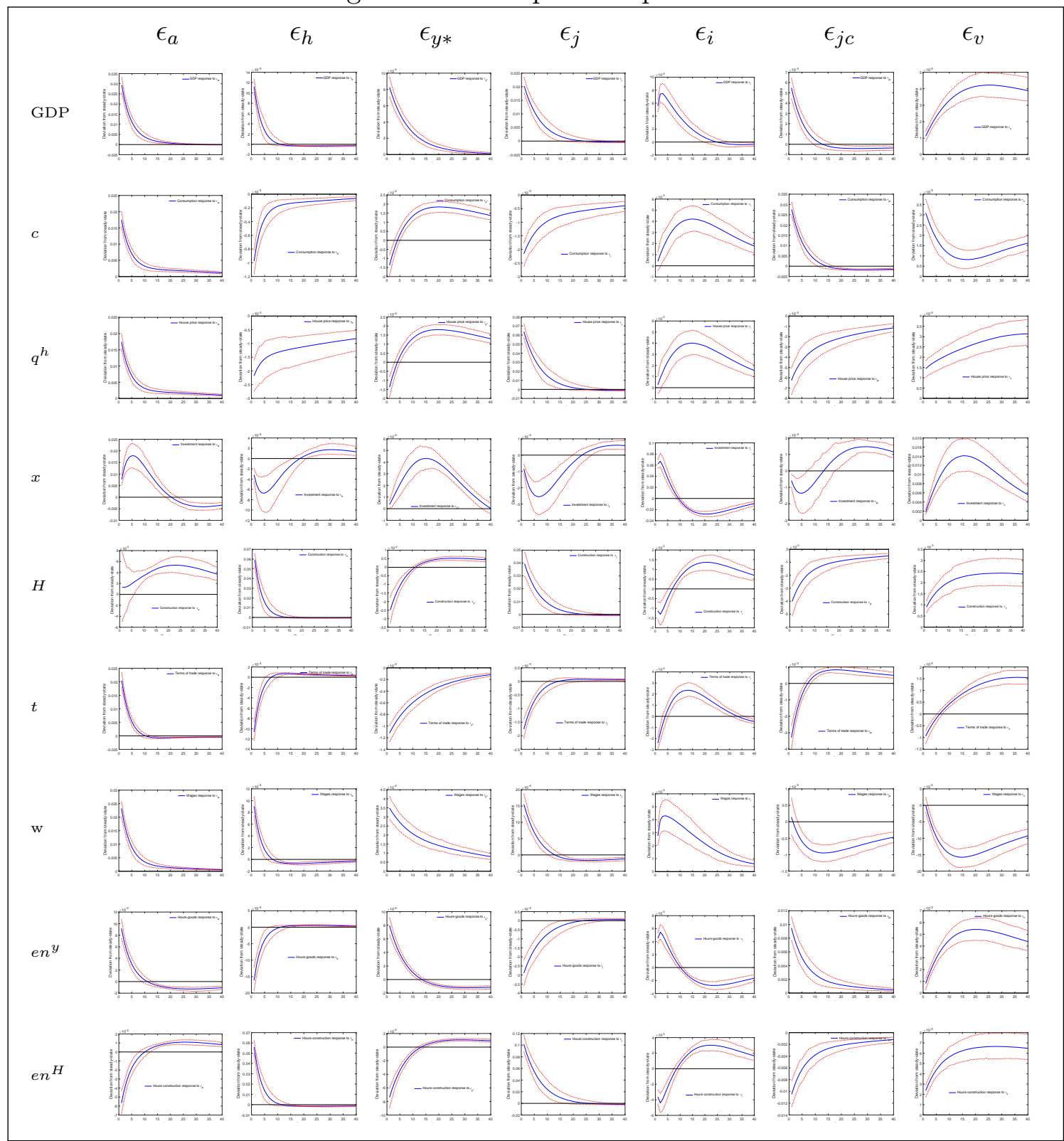

Note: The horizontal axes is measured in quarters. Variables as defined in table 3.1 and structural shocks as in equations (3.30)-(3.36). 


\section{D Historical shock decompositions}

Figure 3.D.1: Shock decomposition - Log GDP per capita

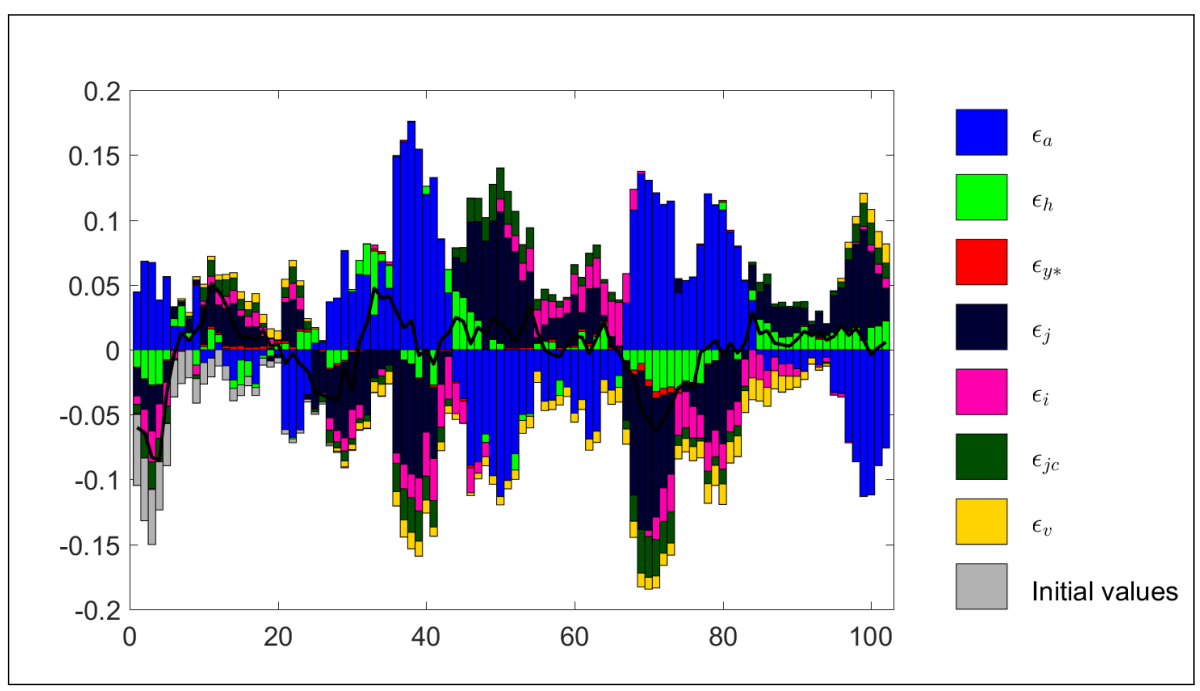

Figure 3.D.2: Shock decomposition - Log consumption per capita

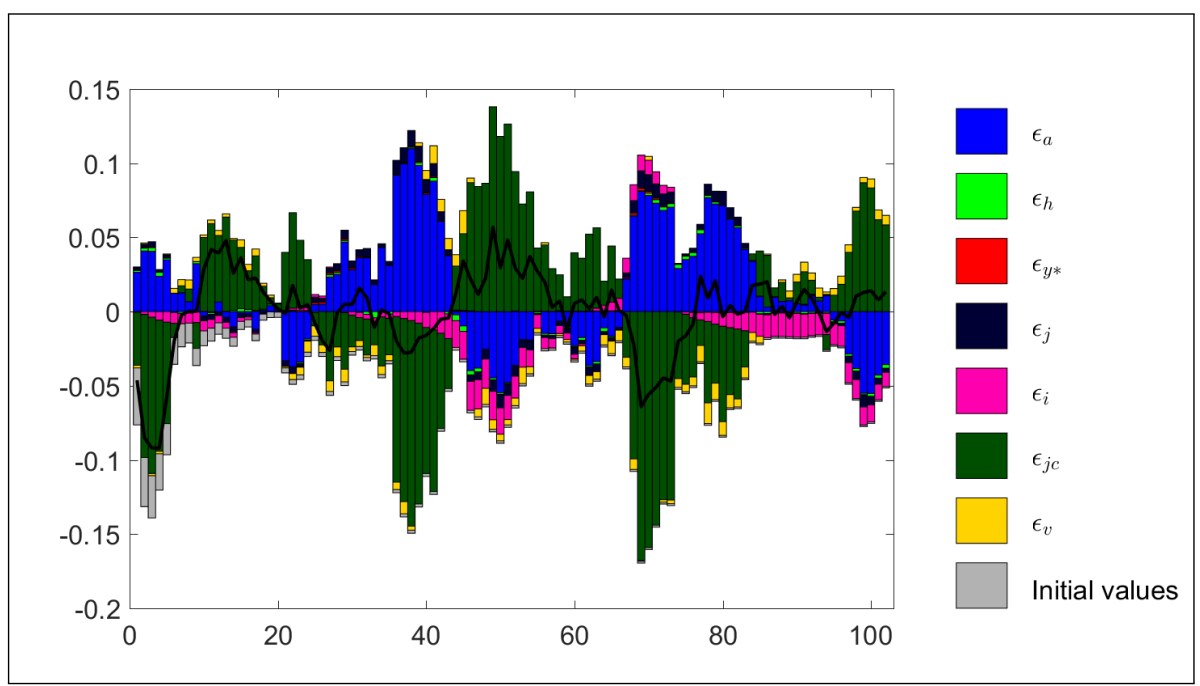


Figure 3.D.3: Shock decomposition - Log investment per capita

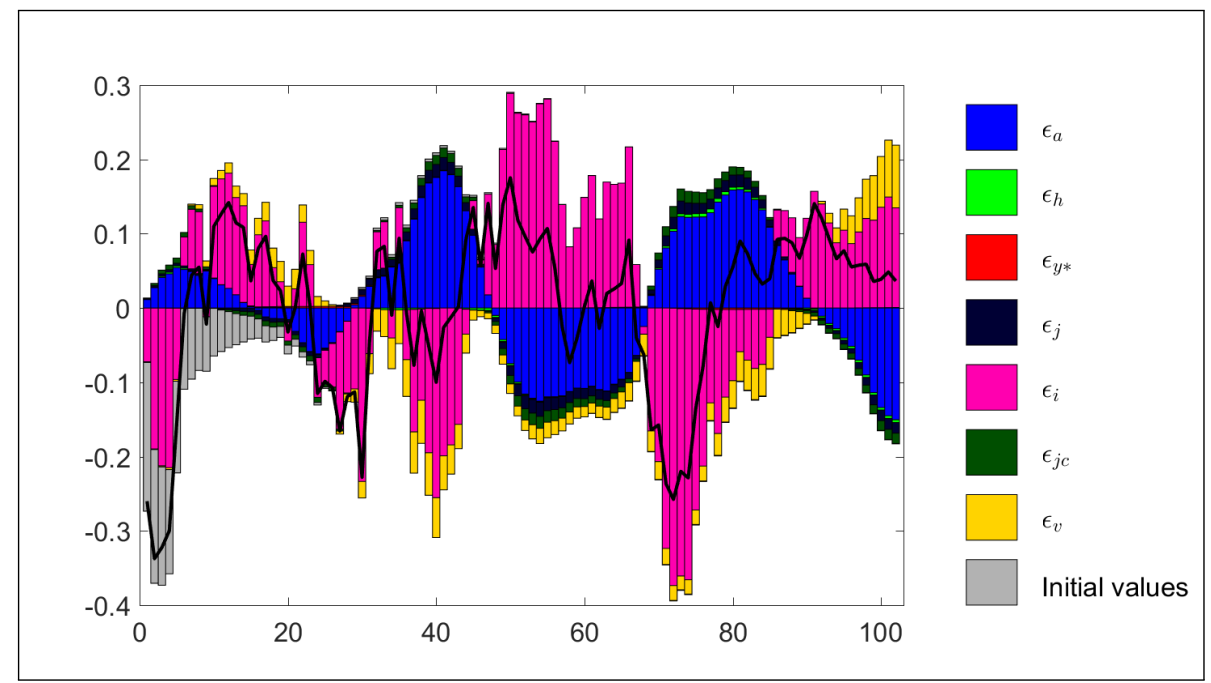

Figure 3.D.4: Shock decomposition - Log residential investment per capita

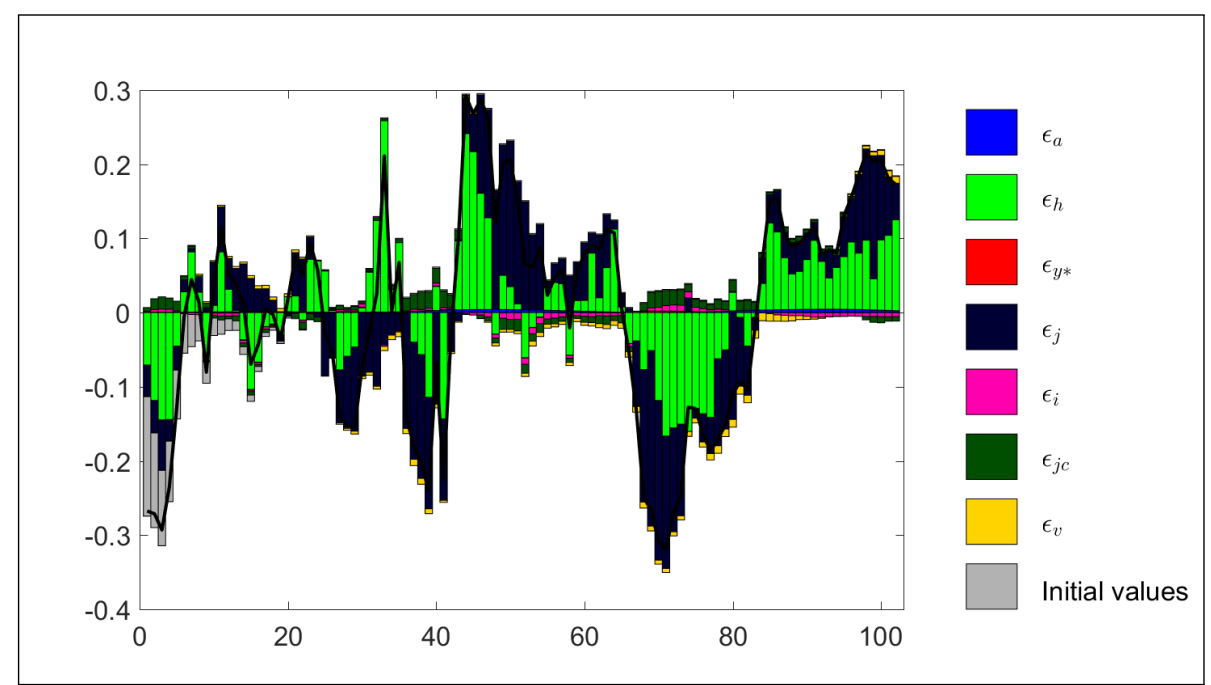


Figure 3.D.5: Shock decomposition - Real house price

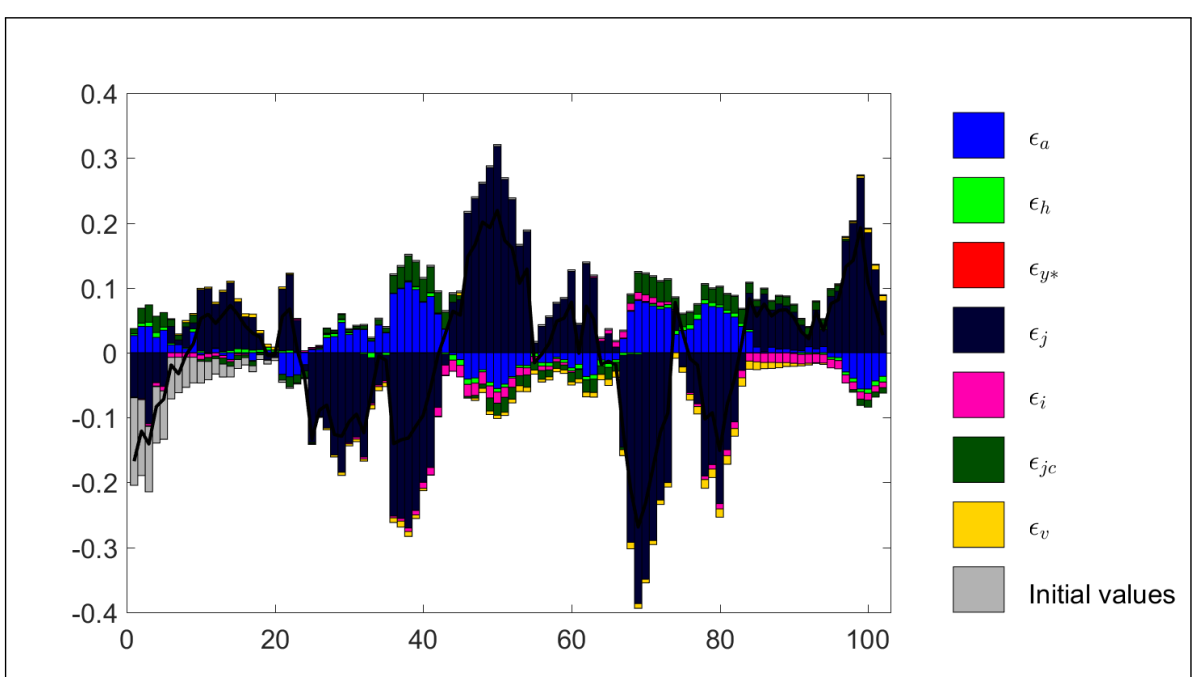

Note: The foreign demand shock and the migration shock explain all of world GDP and migration respectively, and hence are not depicted. 


\section{E Smoothed shocks}

Figure 3.E.1: Smoothed shocks
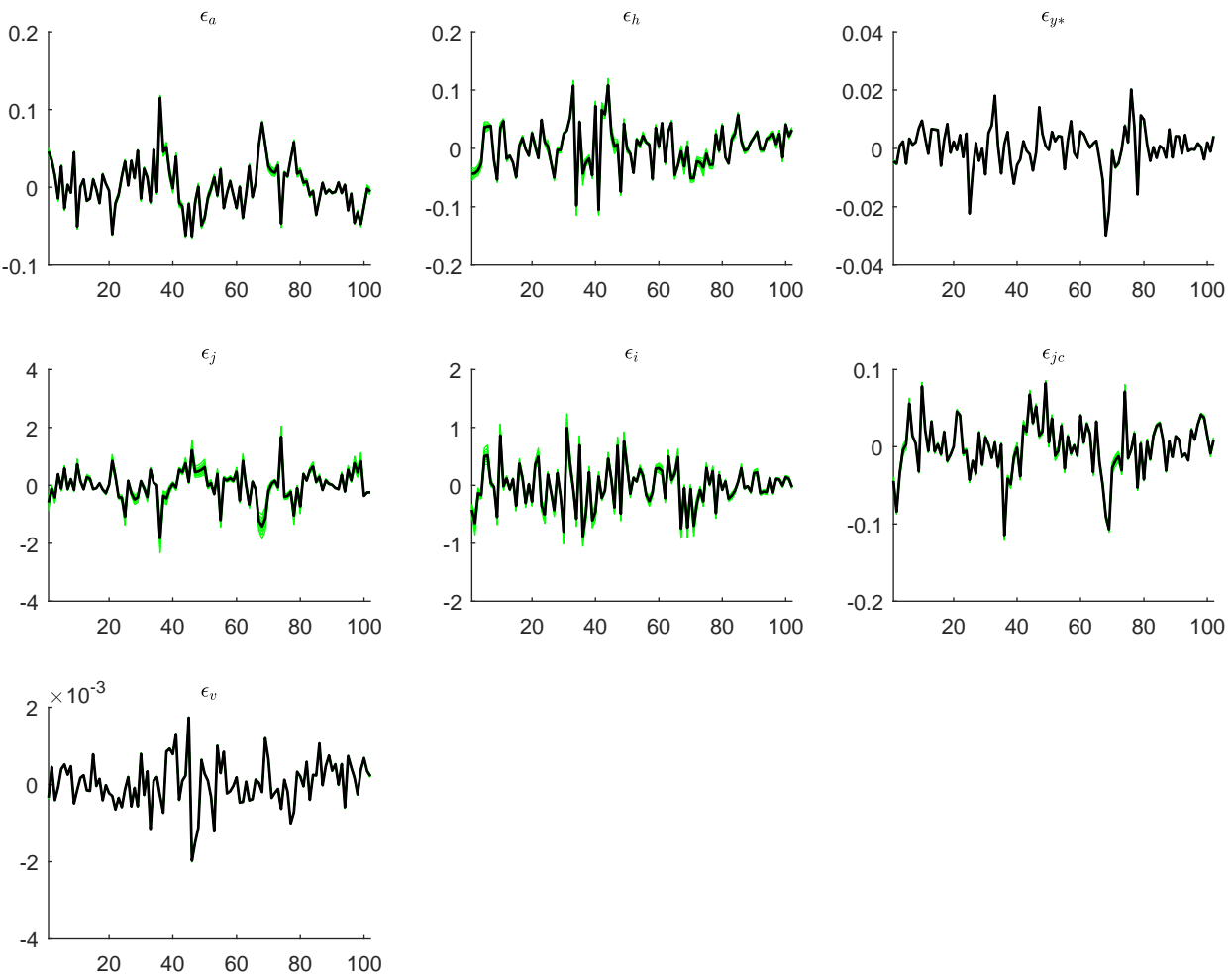

Note: These are the smoothed structural shocks obtained from the Kalman filter. 


\section{Chapter 4}

\section{Financial frictions and investment-specific technology shocks*}

\subsection{Introduction}

Do shocks to investment drive the business cycle? A number of papers over the last decade suggest that investment shocks account for the majority of the variation in key macroeconomic aggregates. ${ }^{1}$ The role of investment shocks has also come into renewed focus following the recent financial crisis. Financial intermediation affects the transformation of savings into usable, installed capital. Likewise, investment shocks affect the economy's ability to transform consumption goods into productive capital and thus play a parallel role to the process of financial intermediation. Justiniano et al. (2011), for example, draw an explicit link between shocks to the marginal efficiency of investment and credit risk spreads. Credit spreads imply the existence of a material financial friction, yet the model in Justiniano et al. (2011) has no such friction.

*This chapter was published as Kamber G., C. Smith and C. Thoenissen (2015) Financial frictions and the role of investment-specific technology shocks in the business cycle, Economic Modelling, 51, 571-582.

${ }^{1}$ See for example Fisher (2006) and Altig et al. (2011) for evidence from structural vector autoregressions and Justiniano et al. (2010, 2011) for DSGE based evidence. For an emerging market context see Araújo (2012). 
Our principal aim in this chapter is to investigate the role and transmission mechanism of investment shocks in the presence of financial frictions. More specifically, we introduce a collateral constraint, similar to that of Kiyotaki and Moore (1997) and Gerali et al. (2010), into the model of Smets and Wouters (2007). We introduce patient households and impatient entrepreneurs into the model, as per Iacoviello (2005) and Iacoviello and Neri (2010). Agents with differential levels of patience also featured in Kiyotaki and Moore (1997). This heterogeneity provides a role for credit, with the impatient entrepreneurs borrowing from the patient households, and ensures that the collateral constraint binds.

Using a data set from the United States (US) that extends from 1954Q3 through to 2011Q4, we estimate our amended model and compute the contribution of structural shocks to the cyclical variation of output, investment, consumption, and so on. We demonstrate that the introduction of a financial friction in the form of a collateral constraint materially alters which shocks are thought to be the most important drivers of the business cycle. The intuition behind our result is simple: a positive investment shock lowers the relative price of capital goods, Tobin's q, and leads to an investment boom. However, when entrepreneurs are subject to binding collateral constraints, a reduction in the value of installed capital reduces the value of collateral and thus reduces the amount an entrepreneur can borrow. As a result, the initial response of investment to a positive investment shock is attenuated by the decline in available credit. In the presence of a collateral constraint the increase in investment cannot be financed via increased borrowing and is therefore accompanied by a decline in entrepreneurial consumption. Consequently, investment shocks do not generate the positive correlation between consumption and investment that is observed in the data.

In our model, the shock affecting the cost of borrowing - the risk premium or consumption shock - is a major driver of cyclical fluctuations in output and other macroeconomic variables. This risk premium shock accounts for around half of the variation in output and consumption, and 40 percent of the variation in investment and interest rates. There is also a striking conformity between the estimated risk premium shock and the US business cycle. ${ }^{2}$ The

${ }^{2}$ See Figure 4.2 . 
collateral constraint also has a material effect on the transmission of risk premium shocks. Contrary to the transmission mechanism of investment shocks described above, a stimulatory risk premium shock causes demand to rise and Tobin's q to increase. This implies that entrepreneurs face a looser borrowing constraint, and thus the impact of the risk premium shock is amplified for both consumption and investment.

As in this chapter, Christiano et al. (2011) and Christiano et al. (2012) observe that the contribution of IST shocks to the variance of GDP is diminished when a financial friction is introduced into the model. Our work differs from those papers in two main respects. First, we have a collateral constraint rather than an external finance premium as our financial friction. Second, in the above papers the IST shock remains an important driver of GDP dynamics except when financial variables are included as observables, whereas in our model - even with just the standard Smets-Wouters observables - the contribution of the IST shock to cyclical dynamics is largely annihilated.

The remainder of this chapter is structured as follows. Section 4.2 outlines the model used in the analysis. The model closely follows that of Smets and Wouters (2007) and Justiniano et al. (2010), but we add impatient entrepreneurs who are collateral constrained. Section 4.3 discusses the estimation of the model. Section 4.4 looks at the role of investment specific technology (IST) and risk premium shocks as cyclical drivers. ${ }^{3}$ In section 4.5 and section 4.6, we discuss our results and their robustness.

\subsection{Model}

Our model is based on the familiar New Keynesian model put forward by Smets and Wouters (2007). Households consume (and save) and supply labour. The household income that underpins consumption and saving is obtained from wages, and from dividend streams from owning the firms that produce final goods. Households smooth consumption over time by investing

\footnotetext{
${ }^{3}$ We refer to investment specific technology shocks in the spirit of Smets and Wouters (2007). Other authors (such as Justiniano et al. 2011) make a distinction between IST shocks, which affect the transformation of consumption goods into investment, and shocks to the marginal efficiency of investment (MEI shocks), which affect the transformation of investment into productive capital.
} 
in deposits issued by competitive financial intermediaries. The model has various nominal and real frictions including price and wage rigidities (with backward inflation indexation), habit formation in consumption, and adjustment costs for investment. The model also has variable capital utilization and fixed costs.

We modify the baseline Smets-Wouters model by introducing entrepreneurial agents who are subject to a borrowing constraint. ${ }^{4}$ Introducing an additional agent into the model provides scope for borrowing and lending in the steady state. We assume that borrowing is limited to a fraction $\chi$ of the present value of the future capital stock owned by the entrepreneur. Mendoza (2006) provides a general specification for collateral constraints nesting the one employed here. Our approach is similar to the 'margin constraint' in Aiyagari and Gertler (1999), which hinges on the value of capital owned. Debt is one-period, so the stock of capital financed by household lending to the entrepreneurs needs to to be re-financed each period.

We adopt a borrowing constraint because it is a parsimonious financial friction, and has a pedigree in theoretical models dating back to at least Kiyotaki and Moore (1997). Furthermore, empirical evidence indicates that collateralization of debt is ubiquitous (see for example Berger and Udell 1990, Harhoff and Korting 1998 and Jimenez et al. 2006); collateral requirements are consistent with the notion that entrepreneurs' borrowing capacity is constrained by the value of their assets.

Entrepreneurs are responsible for all investment. We assume that entrepreneurs have a higher rate of time preference than households and are therefore more impatient. The entrepreneurs' impatience causes the collateral constraint to be binding even in steady state, unlike Mendoza (2008). Entrepreneurial impatience means that entrepreneurs can beneficially exchange current consumption for future consumption by borrowing from households. This intertemporal substitution is enabled by investment in capital goods. All agents, both households and entrepreneurs, are subject to the same stochastic shocks, and thus there is no idiosyncratic risk to insure away.

\footnotetext{
${ }^{4}$ Lombardo and McAdam (2012) also introduce borrowing constraints into the SmetsWouters model, but in their model, the constraint binds for households, while firms are subject to costly state verification.
} 
As discussed by Iacoviello (2005), the return to investment exceeds the return to savings so that the collateral constraint is binding, but we do not want entrepreneurs to postpone consumption to self-fund all of the desired investment, which is prevented by the entrepreneur's impatience.

Entrepreneurs are the agents who own the capital stock. They finance consumption and investment expenditure by renting out capital goods to final goods producers and by borrowing from households, via notional financial intermediaries.

In our description of the model below, we limit our discussion to those parts of the model that differ from Smets and Wouters (2007), focusing on the decision problems of households and entrepreneurs. A full set of linearized model equations is presented in Appendix 4.A.

\subsubsection{Households}

The representative household maximizes the following utility function:

$$
E_{t} \sum_{s=0}^{\infty} \beta^{s}\left[\frac{1}{1-\sigma_{c}}\left(C_{j, t+s}-h C_{t-1+s}\right)^{1-\sigma_{c}} \exp \left(\frac{\sigma_{c}-1}{1+\sigma_{l}} L_{j, t+s}^{1+\sigma_{l}}\right)\right]
$$

subject to

$$
C_{j, t}+\frac{B_{j, t}}{P_{t}}=\Pi_{j, t}+W_{j, t} L_{j, t}+\frac{R_{t-1}^{f}}{\pi_{t}} \frac{B_{j, t-1}}{P_{t-1}}
$$

The $j^{\text {th }}$ household maximizes utility by choosing consumption at time $t, C_{j, t}$, and hours worked $L_{j, t}$. $\beta$ is the discount factor; $h$ dictates the degree of habit persistence; $\sigma_{l}$ is the elasticity of substitution with respect to the real wage; and $\sigma_{c}$ in conjunction with the habit term determines the intertemporal substitution elasticity for households. The flow constraint has consumption and real deposits $\left(B_{j, t} / P_{t}\right)$ equal to profits, $\Pi_{j, t}$, labour income (real wages $W_{j, t}$ multiplied by hours worked) and the value of real deposits from last period scaled up by the gross effective nominal interest rate $R_{t-1}^{f}$ divided by the gross inflation rate, $\pi_{t}$. The gross effective nominal interest rate is defined as $R_{t}^{f} \equiv R_{t} \varepsilon_{c, t}$ where $\varepsilon_{c, t}$ is a risk premium shock, as in Smets and Wouters (2007), and $R_{t}$ is the gross risk free policy rate.

The household's first order conditions for consumption and deposits are 
summarized by the following set of equations. The marginal utility of consumption at time $t$, denoted $\lambda_{t}$, is:

$$
\lambda_{j, t}=\exp \left(\frac{\sigma_{c}-1}{1+\sigma_{l}} L_{j, t}^{1+\sigma_{l}}\right)\left(C_{j, t}-h C_{t-1}\right)^{-\sigma_{c}} .
$$

The Euler equation for households can then be represented as:

$$
\lambda_{j, t}=\beta E_{t}\left(\lambda_{j, t+1} \frac{R_{t}^{f}}{\pi_{t+1}}\right)
$$

The savings, or deposits of the household, $B_{t} / P_{t}$, are lent to entrepreneurs, who use these funds to purchase capital goods. These capital goods are rented out to final goods-producing firms (which are in turn owned by the households).

\subsubsection{Entrepreneurs}

The representative entrepreneur maximizes the expected utility:

$$
\underset{t}{\mathrm{E}} \sum_{s=0}^{\infty} \beta_{e}^{s}\left[\frac{1}{1-\sigma_{e}}\left(C_{j, t+s}^{e}-h_{e} C_{t-1+s}^{e}\right)^{1-\sigma_{e}}\right]
$$

where $C^{e}$ denotes entrepreneurial consumption. Entrepreneurs are subject to the following budget constraint:

$C_{j, t}^{e}+Q_{t} K_{j, t}=\frac{B_{j, t}}{P_{t}}-\frac{R_{t-1}^{f}}{\pi_{t}} \frac{B_{j, t-1}}{P_{t-1}}+R_{t}^{k} Z_{t} K_{j, t-1}-a\left(Z_{t}\right) K_{j, t-1}+Q_{t}(1-\delta) K_{j, t-1}+\Pi_{t}^{e}$

In each period, the entrepreneur purchases consumption goods $C_{j, t}^{e}$ and new capital stock, $K_{j, t}$, at price $Q_{t}$. These purchases are financed by: net borrowing from households $\left(\frac{B_{j, t}}{P_{t}}-\frac{R_{t-1}^{f}}{\pi_{t}} \frac{B_{j, t-1}}{P_{t-1}}\right)$; rental income on capital goods net of capital utilization costs $\left(R_{t}^{k} Z_{t} K_{j, t-1}-a\left(Z_{t}\right) K_{j, t-1}\right)$, where $z_{t}$ is the utilization rate; the proceeds from selling last period's capital stock net of depreciation $\left(Q_{t}(1-\delta) K_{j, t-1}\right)$; and profit from the intermediate production of capital $\left(\Pi_{t}^{e}\right)$. Because entrepreneurs are more impatient than households, 
they face the following borrowing constraint on their degree of leverage:

$$
\frac{R_{t}^{f}}{\pi_{t+1}} \frac{B_{j, t}}{P_{t}}=\chi \underset{t}{\mathrm{E}} Q_{t+1} K_{j, t}
$$

where $\chi$ is the loan-to-value ratio (LVR), which dictates the maximum permissible leverage ratio. This constraint is on the future value of capital, hence $\mathrm{E}_{t} Q_{t+1}$, because any default and required loan recovery will occur in the future. Because of the assumption that $\beta>\beta_{e}$, the constraint is always binding in the neighborhood of the steady state.

The optimality conditions for the entrepreneur's consumption, borrowing, capital purchases, and capital utilization are as follows:

$$
\begin{gathered}
\left(C_{j, t}^{e}-h_{e} C_{t-1}^{e}\right)^{-\sigma_{e}}-\lambda_{j, t}^{e}=0 \\
\lambda_{j, t}^{e}-\beta_{e} E_{t} \lambda_{j, t+1}^{e} \frac{R_{t}^{f}}{\pi_{t+1}}-\lambda_{j, t}^{B} \frac{R_{t}^{f}}{\pi_{t+1}}=0 \\
Q_{t}=\frac{\lambda_{j, t}^{B}}{\lambda_{j, t}^{e}} \chi Q_{t+1}+\beta_{e} \underset{t}{\mathrm{E}} \frac{\lambda_{j, t+1}^{e}}{\lambda_{j, t}^{e}}\left[R_{t+1}^{k} Z_{t+1}-a\left(Z_{t+1}\right)+Q_{t+1}(1-\delta)\right] \\
R_{t}^{k}=a^{\prime}\left(Z_{t}\right)
\end{gathered}
$$

where $\lambda^{e}$ and $\lambda^{B}$ are the Lagrange multipliers on the flow and borrowing constraints respectively, $R_{t}^{k}$ is the return on capital and, as before, $Z_{t}$ is capital utilization.

The presence of $\lambda^{B}$ in the first order conditions represents the effects of the borrowing constraint on entrepreneurs' allocation of consumption and capital purchases. Consider, for example, a case where the borrowing constraint is exogenously relaxed. This results in a decline in the shadow value of the constraint, $\lambda^{B}$. For constant real interest rates, the Euler equation suggests that a looser borrowing constraint would be associated with higher consumption. Likewise, for a constant path of the effective interest rate, a looser borrowing constraint implies a higher value of installed capital, Q, and thus higher investment. 


\section{Capital producers}

The capital stock is produced by firms, wholly owned by the entrepreneurs. The $j^{\text {th }}$ representative capital-producing firm maximizes the following profit function:

$$
\underset{t}{\mathrm{E}} \sum_{s=0}^{\infty} \Lambda_{t+s}^{e}\left[Q_{t+s} \Delta x_{j, t+s}-I_{j, t+s}\right]
$$

where $\Lambda_{t}^{e}$ is the stochastic discount factor of the owner, in this case the entrepreneur, and net capital accumulation is defined as:

$$
\Delta x_{j, t}=K_{j, t}-(1-\delta) K_{j, t-1}=\varepsilon_{\mu, t}\left(1-S\left(I_{j, t}, I_{j, t-1}\right)\right) I_{j, t}
$$

where $\delta$ is the depreciation rate, $\varepsilon_{\mu, t}$ is an investment-specific shock, and the function $\left.S\left(I_{j, t}, I_{j, t-1}\right)\right) I_{j, t}$ captures investment adjustment costs. The investment adjustment cost function is quadratic in the ratio of investment to its lag. Substituting (4.13) into (4.12) yields:

$$
\underset{t}{\mathrm{E}} \sum_{s=0}^{\infty} \Lambda_{t+s}^{e}\left[Q_{t+s} \varepsilon_{\mu, t+s}\left(1-S\left(I_{j, t+s}, I_{j, t+s-1}\right)\right) I_{j, t+s}-I_{j, t+s}\right]
$$

Assuming that the adjustment cost function $S\left(I_{j, t}, I_{j, t-1}\right)$ takes the form $\frac{\kappa}{2}\left(\frac{I_{j, t}}{I_{j, t-1}}-\gamma\right)^{2}$, where $\gamma$ is the gross steady state growth rate of the economy, the optimality condition for investment is given by:

$$
\begin{aligned}
1 & =Q_{t} \varepsilon_{\mu, t}\left[\left(1-\frac{\kappa}{2}\left(\frac{I_{j, t}}{I_{j, t-1}}-\gamma\right)^{2}\right)-\kappa\left(\frac{I_{j, t}}{I_{j, t-1}}-\gamma\right) \frac{I_{j, t}}{I_{j, t-1}}\right] \\
& +\beta_{e} \underset{t}{\mathrm{E}} \frac{\lambda_{t+1}^{e}}{\lambda_{t}^{e}} Q_{t+1} \varepsilon_{\mu, t+1}\left[\kappa\left(\frac{I_{j, t+1}}{I_{j, t}}-\gamma\right)\left(\frac{I_{j, t+1}}{I_{j, t}}\right)^{2}\right]
\end{aligned}
$$

Adjustment costs dampen the response of investment to various shocks and play an important role in the dynamics of Tobin's q - the relative price of firms' collateral in our model. 


\subsubsection{The rest of the model}

The rest of the model directly follows Smets and Wouters (2007) and thus we only provide a very brief description. A complete set of linearized model equations is presented in Table 4.A.1 in Appendix 4.A.

Output of final goods is a function of effective capital, labour and technology. Final goods producers rent capital services with a given degree of utilization from entrepreneurs, and labour services from household unions.

Goods and labour markets are monopolistically competitive with both prices and wages being set in a time-dependent manner as put forward by Calvo (1983), albeit with partial indexation to past inflation for those price and wage setters not called upon to re-price in a given time period.

Government spending is simply modeled as a stochastic share of GDP. Monetary policy is modeled by a generalized Taylor-type interest rate rule that links the current period policy rate to its lag, to deviations of the current period inflation rate from target, to deviations in the output gap, and to changes in the growth rate of the output gap.

The output gap is defined as the difference between output in the sticky price allocation of the model and output corresponding to a flexible price allocation. In the flexible price allocation there are no nominal rigidities in either price or wage setting, and hence there is no role for monetary policy.

\subsubsection{Shocks}

There are seven shocks perturbing the economy. The risk premium $\left(\varepsilon_{c, t}\right)$ and investment specific technology shock $\left(\varepsilon_{\mu, t}\right)$, discussed above, are augmented with shocks to total factor productivity $\left(\varepsilon_{a, t}\right)$, the share of government spending in GDP $\left(\varepsilon_{g, t}\right)$, the interest rate rule $\left(\varepsilon_{r, t}\right)$, and shocks to the price and wage Phillips curves $\left(\varepsilon_{p, t}\right.$ and $\left.\varepsilon_{w, t}\right) .{ }^{5}$ These shocks all exhibit some degree of

\footnotetext{
${ }^{5}$ The flexible price allocation used to construct the output gap is not affected by either $\varepsilon_{r, t}, \varepsilon_{p, t}$ or $\varepsilon_{w, t}$.
} 
persistence, as described in the following equations:

$$
\begin{aligned}
\varepsilon_{c, t} & =\rho_{c} \varepsilon_{c, t-1}+\zeta_{c, t} \\
\varepsilon_{\mu, t} & =\rho_{\mu} \varepsilon_{\mu, t-1}+\zeta_{\mu, t} \\
\varepsilon_{a, t} & =\rho_{a} \varepsilon_{a, t-1}+\zeta_{a, t} \\
\varepsilon_{g, t} & =\rho_{g} \varepsilon_{g, t-1}+\zeta_{g, t}+\rho_{(g, a)} \zeta_{a, t} \\
\varepsilon_{r, t} & =\rho_{r} \varepsilon_{r, t-1}+\zeta_{r, t} \\
\varepsilon_{p, t} & =\rho_{p} \varepsilon_{p, t-1}+\zeta_{p, t}-\rho_{(p, \zeta)} \zeta_{p, t-1} \\
\varepsilon_{w, t} & =\rho_{w} \varepsilon_{w, t-1}+\zeta_{w, t}-\rho_{(w, \zeta)} \zeta_{w, t-1}
\end{aligned}
$$

The various autoregressive and moving average (MA) coefficients are represented by $\rho$. Following Smets and Wouters (2007), we include a feedback term between the innovation in technology and government spending, $\rho_{(g, a)}$, in the shock term for exogenous government spending, as well as MA terms in the price and wage shocks to capture high frequency fluctuations in price and wage dynamics. The innovations $\zeta_{j, t}$ are normal, independent and identically distributed.

\subsubsection{An alternative model}

To isolate the effects of borrowing constraints on the business cycle, we estimate two versions of our model: the model presented above, and an alternative model where entrepreneurs are identical to households in terms of their rate of time preference and thus do not face borrowing constraints. This alternative is essentially the model put forward by Smets and Wouters (2007).

\subsection{Bayesian estimation}

The following seven observables are used to estimate the two versions of the model: the growth rates of GDP, aggregate consumption, and investment; real wages; inflation; the short-term nominal interest rate; and hours worked. Given that we have seven stochastic shocks in the model, we avoid stochastic singularity. The data used to estimate the models are described in Appendix 
4.B. We denote 'aggregate' consumption as $C^{a}$ since it corresponds to the sum of household and entrepreneurial consumption in our model. As in Justiniano et al. (2010), consumption corresponds to private consumption of non-durable goods, while investment is defined as the sum of gross domestic private investment and consumption of durable goods. The models are estimated using standard Bayesian techniques. For the most part the priors for the model are the same as those employed by Smets and Wouters. There are two innocuous caveats to this statement. First, we use a Gamma prior instead of a Normal prior for the labour-disutility parameter, $\sigma_{l}$, though with the same mean and variance used in Smets and Wouters. ${ }^{6}$ Second, we estimate the household's discount rate using a Gamma prior with a mean of 0.25 and a standard deviation of 0.1, though the data are found to be somewhat uninformative for these priors. Other authors such as Iacoviello (2005) and Iacoviello and Neri (2010) calibrate this parameter directly.

The model with borrowing constraints has two parameters without analogues in the original Smets-Wouters model: (i) the loan-to-value ratio, $\chi$, and (ii) the gap between the discount rates of the households and entrepreneurs, $\tilde{\beta}$. Given that the LVR is a device to ensure that entrepreneur's have equity in their investment ventures, the LVR is assumed to fall within $(0,1)$. More specifically the prior for the LVR is a Beta distribution with mean 0.5 and standard deviation of 0.15. Iacoviello and Neri (2010) calibrate the LVR to be 0.85 , suggesting that it is difficult to estimate without data on debt and housing holdings of credit-constrained households. Our mean posterior parameter estimates for the LVR are close to our prior value of 0.5 , but the data are somewhat informative, indicating that the probability mass should be more tightly grouped around the mean value. Indeed, when taking the model to a shortened data sample, starting in the post-Volcker period, we obtain a posterior mean of 0.7 for the same prior.

The prior distribution for the discount rate gap, $\tilde{\beta}$, is a Gamma distribution with a mean of 1 and a standard deviation of 0.5 . This prior distribution implicitly encompasses the calibrated discount factors for impatient borrowers used in Iacoviello (2005) and Iacoviello and Neri (2010), which range

\footnotetext{
${ }^{6}$ In estimation over a smaller sub-sample, positive probability mass was assigned to negative parameter values, which we rule out on a priori theoretical grounds.
} 
from 0.98 to 0.97 . Gerali et al. (2010) estimate a similar model, but do not attempt to estimate either $\chi$ or $\tilde{\beta}$. Iacoviello provides greater discussion of plausible discount factors, and cites a number of papers on cross-sectional variation in discount factors (Carroll and Samwick 1997, for example, suggest that the plausible range for discount factors is between 0.91 and 0.99). While our prior range does not fully encompass this cross-sectional variation we think it provides a sufficiently broad range for what one might assume is the average impatient entrepreneur.

Finally, we calibrate the depreciation rate to 0.025 and the share of government spending in GDP to 0.22. Following Smets and Wouters (2007), we set the Kimball aggregator parameters, $\epsilon_{p}$ and $\epsilon_{w}$, to 10 and calibrate the steady state wage mark-up to 1.5 .

Table 4.1: Estimation results for parameters and shock processes of model with borrowing constraints: 1954Q3 2011Q4

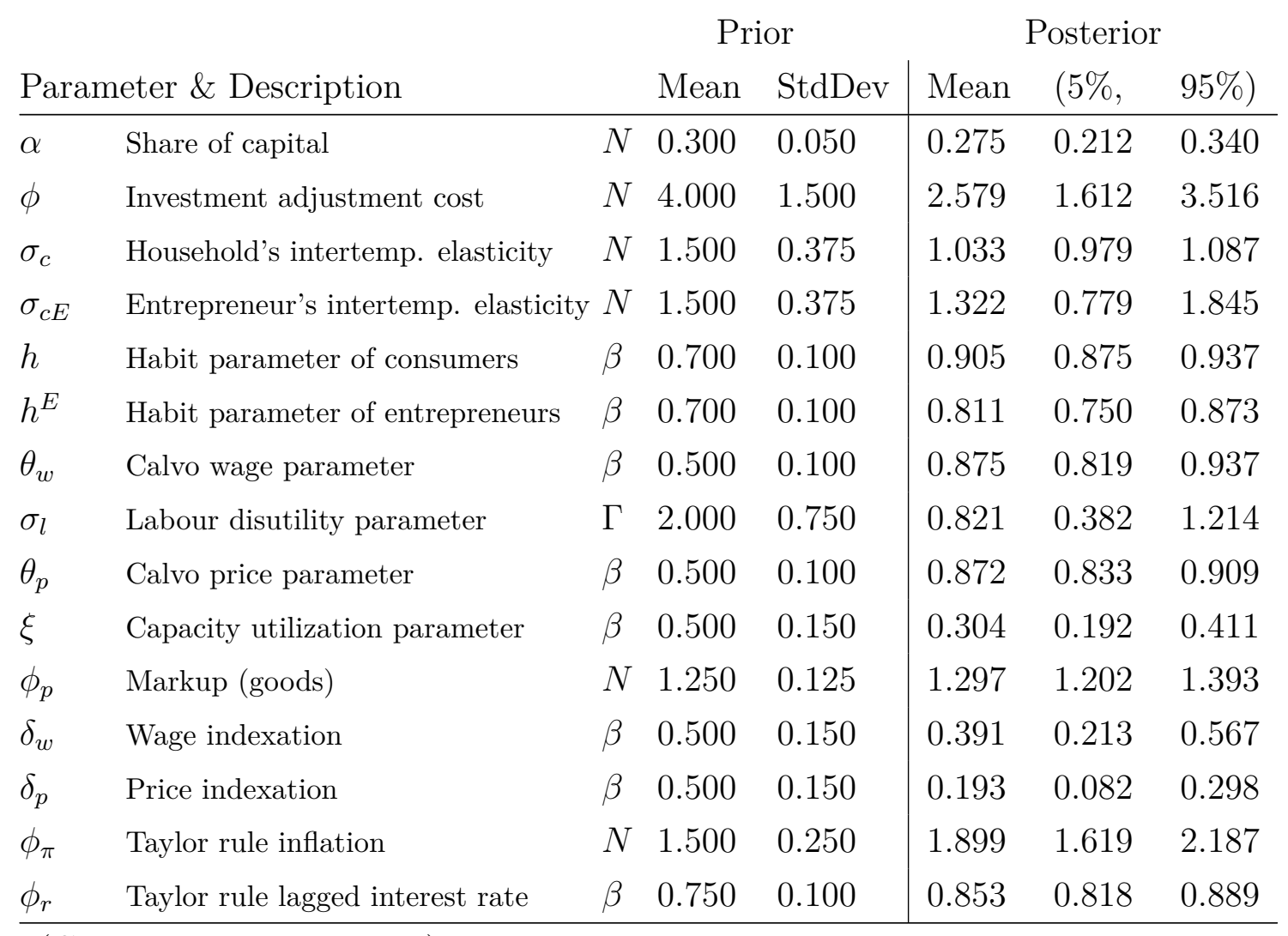

(Continued on next page.) 


\begin{tabular}{|c|c|c|c|c|c|c|c|}
\hline \multirow{2}{*}{\multicolumn{3}{|c|}{ Parameter \& Description }} & \multicolumn{2}{|c|}{ Prior } & \multicolumn{3}{|c|}{ Posterior } \\
\hline & & & Mean & StdDev & Mean & $(5 \%$ & $95 \%)$ \\
\hline$\overline{\phi_{x}}$ & Taylor rule output gap & $N$ & 0.125 & 0.050 & 0.074 & 0.044 & 0.106 \\
\hline$\phi_{\Delta x}$ & Taylor rule output gap growth rate & $N$ & 0.125 & 0.050 & 0.245 & 0.210 & 0.282 \\
\hline $\bar{\pi}$ & Steady state inflation & $\Gamma$ & 0.625 & 0.100 & 0.835 & 0.734 & 0.943 \\
\hline$\frac{100(1-\beta}{\beta}$ & Discount rate (percent) & $\Gamma$ & 0.250 & 0.100 & 0.250 & 0.092 & 0.398 \\
\hline ltv & Loan to value ratio & $\beta$ & 0.500 & 0.150 & 0.510 & 0.322 & 0.709 \\
\hline$\tilde{\beta}$ & Entrepreneurs discount less H'hlds & $\Gamma$ & 1.000 & 0.500 & 0.985 & 0.222 & 1.717 \\
\hline lss & Log steady state hours & $N$ & 0.000 & 2.000 & -0.580 & -2.628 & 1.504 \\
\hline$\gamma$ & Steady state growth rate (percent) & $N$ & 0.400 & 0.100 & 0.470 & 0.443 & 0.495 \\
\hline$\rho_{a}$ & AR parameter technology & $\beta$ & 0.500 & 0.200 & 0.970 & 0.960 & 0.982 \\
\hline$\rho_{c}$ & AR parameter risk premium & $\beta$ & 0.500 & 0.200 & 0.866 & 0.827 & 0.904 \\
\hline$\rho_{g}$ & AR parameter exog. demand & $\beta$ & 0.500 & 0.200 & 0.990 & 0.982 & 0.997 \\
\hline$\rho_{i}$ & AR parameter investment & $\beta$ & 0.500 & 0.200 & 0.192 & 0.123 & 0.264 \\
\hline$\rho_{r}$ & AR parameter interest rate & $\beta$ & 0.500 & 0.200 & 0.134 & 0.051 & 0.212 \\
\hline$\rho_{p}$ & AR parameter price markup & $\beta$ & 0.500 & 0.200 & 0.945 & 0.906 & 0.982 \\
\hline$\rho_{w}$ & AR parameter wage markup & $\beta$ & 0.500 & 0.200 & 0.957 & 0.928 & 0.988 \\
\hline$\rho_{e p}$ & MA parameter price markup & $\beta$ & 0.500 & 0.200 & 0.888 & 0.818 & 0.954 \\
\hline$\rho_{e w}$ & MA parameter wage markup & $\beta$ & 0.500 & 0.200 & 0.934 & 0.897 & 0.972 \\
\hline$\rho_{g a}$ & Effect of tech shock on exog. Dd. & $N$ & 0.500 & 0.200 & 0.359 & 0.280 & 0.434 \\
\hline$\sigma_{c}$ & Std dev. of risk premium shock & $\Gamma^{-1}$ & 10.100 & 2.000 & 0.387 & 0.341 & 0.430 \\
\hline$\sigma_{w}$ & Std dev. of wage markup shock & $\Gamma^{-1}$ & ${ }^{1} 0.100$ & 2.000 & 0.258 & 0.230 & 0.285 \\
\hline$\sigma_{p}$ & Std dev. of price markup shock & $\Gamma^{-1}$ & ${ }^{1} 0.100$ & 2.000 & 0.141 & 0.122 & 0.160 \\
\hline$\sigma_{r}$ & Std dev. of interest rate shock & $\Gamma^{-1}$ & 0.100 & 2.000 & 0.216 & 0.197 & 0.236 \\
\hline$\sigma_{a}$ & Std dev. of technology shock & & 0.100 & 2.000 & 0.548 & 0.501 & 0.592 \\
\hline$\sigma_{i}$ & Std dev. of investment shock & $\Gamma^{-1}$ & 0.100 & 2.000 & 2.199 & 1.807 & 2.610 \\
\hline$\sigma_{g}$ & Std dev. of exog. demand shock & $\Gamma^{-1}$ & 10.100 & 2.000 & 0.344 & 0.315 & 0.373 \\
\hline
\end{tabular}

Notes: The prior for a parameter is a Normal $(N)$, Beta $(\beta)$, Gamma $(\Gamma)$, or inverseGamma $\left(\Gamma^{-1}\right)$ distribution. Columns 4 and 5 indicate the mean and standard deviation of the prior distribution, and the final three columns report the posterior mean and lower and upper limits of 90 percent Bayesian confidence intervals from the posterior distribution.

Tables 4.1 and 4.2 report the posterior mean and 90 percent posterior probability intervals for the structural parameters and the standard devi- 
ations of the shocks for the model with and without collateral constraints. The reported parameter estimates for the models are based on 900,000 draws of Markov chains. Appendix 4.C illustrates (recursive) 'trace-plots' of deciles from two Markov chains, to provide some degree of confidence that the chains have converged to their stationary (posterior) distributions.

Table 4.2: Estimation results for parameters and shock processes of model without borrowing constraints: 1954Q3 - 2011Q4

\begin{tabular}{|c|c|c|c|c|c|c|c|}
\hline \multirow{2}{*}{\multicolumn{3}{|c|}{ Parameter \& Description }} & \multicolumn{2}{|c|}{ Prior } & \multicolumn{3}{|c|}{ Posterior } \\
\hline & & & Mean & StdDev & Mean & $(5 \%$ & $95 \%)$ \\
\hline$\alpha$ & Share of capital & $N$ & 0.300 & 0.050 & 0.126 & 0.055 & 0.198 \\
\hline$\phi$ & Investment adjustment cost & $N$ & 4.000 & 1.500 & 4.882 & 3.235 & 6.548 \\
\hline$\sigma_{c}$ & Household's intertemp. elasticity & $N$ & 1.500 & 0.375 & 1.358 & 1.205 & 1.499 \\
\hline$h$ & Habit parameter of consumers & $\beta$ & 0.700 & 0.100 & 0.759 & 0.686 & 0.826 \\
\hline$\theta_{w}$ & Calvo wage parameter & $\beta$ & 0.500 & 0.100 & 0.811 & 0.751 & 0.876 \\
\hline$\sigma_{l}$ & Labour disutility parameter & $\Gamma$ & 2.000 & 0.750 & 1.363 & 0.644 & 2.125 \\
\hline$\theta_{p}$ & Calvo price parameter & $\beta$ & 0.500 & 0.100 & 0.765 & 0.716 & 0.818 \\
\hline$\xi$ & Capacity utilization parameter & $\beta$ & 0.500 & 0.150 & 0.711 & 0.567 & 0.840 \\
\hline$\phi_{p}$ & Markup (goods) & $N$ & 1.250 & 0.125 & 1.291 & 1.195 & 1.381 \\
\hline$\delta_{w}$ & Wage indexation & $\beta$ & 0.500 & 0.150 & 0.576 & 0.385 & 0.765 \\
\hline$\delta_{p}$ & Price indexation & $\beta$ & 0.500 & 0.150 & 0.225 & 0.111 & 0.334 \\
\hline$\phi_{\pi}$ & Taylor rule inflation & $N$ & 1.500 & 0.250 & 1.896 & 1.660 & 2.124 \\
\hline$\phi_{r}$ & Taylor rule lagged interest rate & $\beta$ & 0.750 & 0.100 & 0.797 & 0.759 & 0.836 \\
\hline$\phi_{x}$ & Taylor rule output gap & $N$ & 0.125 & 0.050 & 0.076 & 0.049 & 0.103 \\
\hline$\phi_{\Delta x}$ & Taylor rule output gap growth rate & $N$ & 0.125 & 0.050 & 0.211 & 0.171 & 0.251 \\
\hline $\bar{\pi}$ & Steady state inflation & $\Gamma$ & 0.625 & 0.100 & 0.871 & 0.768 & 0.975 \\
\hline$\frac{100(1-\beta)}{\beta}$ & Discount rate (percent) & $\Gamma$ & 0.250 & 0.100 & 0.245 & 0.090 & 0.380 \\
\hline lss & Log steady state hours & $N$ & 0.000 & 2.000 & -0.538 & -2.400 & 1.327 \\
\hline$\gamma$ & Steady state growth rate (percent) & $N$ & 0.400 & 0.100 & 0.450 & 0.420 & 0.482 \\
\hline$\rho_{a}$ & AR parameter technology & $\beta$ & 0.500 & 0.200 & 0.981 & 0.971 & 0.989 \\
\hline$\rho_{c}$ & AR parameter risk premium & $\beta$ & 0.500 & 0.200 & 0.507 & 0.365 & 0.647 \\
\hline$\underline{\rho_{g}}$ & AR parameter exog. demand & $\beta$ & 0.500 & 0.200 & 0.986 & 0.978 & 0.994 \\
\hline
\end{tabular}

(Continued on next page.) 


\begin{tabular}{|c|c|c|c|c|c|c|c|}
\hline \multirow{2}{*}{\multicolumn{3}{|c|}{ Parameter \& Description }} & \multicolumn{2}{|c|}{ Prior } & \multicolumn{3}{|c|}{ Posterior } \\
\hline & & & \multirow{2}{*}{$\frac{\text { Mean }}{0.500}$} & \multirow{2}{*}{$\frac{\text { StdDev }}{0.200}$} & \multirow{2}{*}{$\begin{array}{l}\text { Mean } \\
0.695\end{array}$} & \multirow{2}{*}{$\frac{(5 \%}{0.611}$} & \multirow{2}{*}{$\frac{95 \%)}{0.783}$} \\
\hline$\rho_{i}$ & AR parameter investment & $\beta$ & & & & & \\
\hline$\rho_{r}$ & AR parameter interest rate & $\beta$ & 0.500 & 0.200 & 0.256 & 0.152 & 0.367 \\
\hline$\rho_{p}$ & AR parameter price markup & $\beta$ & 0.500 & 0.200 & 0.964 & 0.942 & 0.985 \\
\hline$\rho_{w}$ & AR parameter wage markup & $\beta$ & 0.500 & 0.200 & 0.962 & 0.941 & 0.986 \\
\hline$\rho_{e p}$ & MA parameter price markup & $\beta$ & 0.500 & 0.200 & 0.820 & 0.744 & 0.910 \\
\hline$\rho_{e w}$ & MA parameter wage markup & $\beta$ & 0.500 & 0.200 & 0.921 & 0.882 & 0.962 \\
\hline$\rho_{g a}$ & Effect of tech shock on exog. Dd. & $N$ & 0.500 & 0.200 & 0.256 & 0.192 & 0.323 \\
\hline$\sigma_{c}$ & Std dev. of risk premium shock & & ${ }^{1} 0.100$ & 2.000 & 1.427 & 0.801 & 1.974 \\
\hline$\sigma_{w}$ & Std dev. of wage markup shock & & ${ }^{1} 0.100$ & 2.000 & 0.261 & 0.234 & 0.289 \\
\hline$\sigma_{p}$ & Std dev. of price markup shock & & ${ }^{1} 0.100$ & 2.000 & 0.121 & 0.100 & 0.142 \\
\hline$\sigma_{r}$ & Std dev. of interest rate shock & & ${ }^{1} 0.100$ & 2.000 & 0.210 & 0.192 & 0.228 \\
\hline$\sigma_{a}$ & Std dev. of technology shock & & ${ }^{1} 0.100$ & 2.000 & 0.565 & 0.518 & 0.615 \\
\hline$\sigma_{i}$ & Std dev. of investment shock & & ${ }^{1} 0.100$ & 2.000 & 0.967 & 0.836 & 1.109 \\
\hline$\sigma_{g}$ & Std dev. of exog. demand shock & $\Gamma^{-}$ & ${ }^{1} 0.100$ & 2.000 & 0.320 & 0.294 & 0.344 \\
\hline
\end{tabular}

Notes: The prior for a parameter is a Normal $(N)$, Beta $(\beta)$, Gamma $(\Gamma)$, or inverseGamma $\left(\Gamma^{-1}\right)$ distribution. Columns 4 and 5 indicate the mean and standard deviation of the prior distribution, and the final three columns report the posterior mean and lower and upper limits of 90 percent Bayesian confidence intervals from the posterior distribution.

The posterior estimates for the common structural parameters in the two models are broadly similar. They suggest a high degree of nominal price and wage rigidity, a significant degree of habit persistence and sluggish investment adjustment. Differences between the two models arise primarily in the size and persistence of investment and risk premium shocks. In the presence of borrowing constraints, investment shocks become more volatile but less persistent. Risk premium shocks, however, are estimated to be less volatile but more persistent. Introducing borrowing constraints also lowers the mean of the posterior estimates of the capital utilization and investment adjustment cost parameters, relative to the model without borrowing constraints.

The additional structure that we have introduced with the two agent types and the borrowing constraint has come at a cost. Like Brzoza-Brzezina and Kolasa (2013), we find that empirical fit is adversely affected by the in- 
Table 4.3: Log marginal data densities

\begin{tabular}{lrr} 
& \multicolumn{2}{c}{ Models } \\
& $\begin{array}{r}\text { No borrowing } \\
\text { constraint }\end{array}$ & $\begin{array}{c}\text { Borrowing } \\
\text { constraint }\end{array}$ \\
\hline Modified Harmonic Mean & -1342.001 & -1413.831 \\
Laplace Approximation & -1340.979 & -1413.943 \\
\hline
\end{tabular}

troduction of the borrowing constraint. Estimates of the (log) marginal data densities of the models with and without the borrowing constraint are reported in Table 4.3. ${ }^{7}$ The modified harmonic mean estimate is based on an average of the draws from the Markov chains, and the Laplace estimate is based on a second order approximation of that log marginal data density (which approximates the data density using a Normal distribution). Bayes factors can be computed from these log marginal data densities, which can then be used to compare the two models (Kass and Raftery, 1995). Kass and Raftery's guidelines to assess the log Bayes factor imply that the data strongly support the model without the borrowing constraint. ${ }^{8}$ What we demonstrate below is that IST shocks are incompatible with borrowing constraints, as implemented in the model. However, to explain financial frictions empirically, alternative structural assumptions are needed, or additional features are required to rehabilitate the model with borrowing constraints.

\subsection{IST and risk premium shocks and the busi- ness cycle}

This section analyzes the key drivers of the business cycle by looking at the variance decomposition of the observables in both version of the model. Table 4.4 reports the contribution of each structural shock to the volatility of the observables for the version of the model without the borrowing constraint.

\footnotetext{
${ }^{7}$ See also Brzoza-Brzezina et al. (2013).

${ }^{8}$ If $2 \log _{e}\left(B_{01}\right)>10$ then the evidence is considered to be 'very strong' in favour of model 0 , where $B_{01}$ is the marginal data density of model zero divided by the marginal data density of model one.
} 
Table 4.4: Variance decomposition of model without borrowing constraints:

\begin{tabular}{|c|c|c|c|c|c|c|c|}
\hline & $\begin{array}{c}\text { Risk } \\
\text { premium }\end{array}$ & $\begin{array}{l}\text { Wage } \\
\text { markup }\end{array}$ & $\begin{array}{l}\text { Price } \\
\text { markup }\end{array}$ & $\begin{array}{l}\text { Monetary } \\
\text { policy }\end{array}$ & $\begin{array}{c}\text { Neutral } \\
\text { Technology }\end{array}$ & IST & Govt. \\
\hline $\begin{array}{l}\text { Output } \\
\text { growth }\end{array}$ & $\begin{array}{c}0.10 \\
{[0.03,0.18]}\end{array}$ & $\begin{array}{c}0.04 \\
{[0.01,0.06]}\end{array}$ & $\begin{array}{c}0.05 \\
{[0.02,0.07]}\end{array}$ & $\begin{array}{c}0.06 \\
{[0.04,0.08]}\end{array}$ & $\begin{array}{c}0.09 \\
{[0.06,0.12]}\end{array}$ & $\begin{array}{c}0.55 \\
{[0.43,0.65]}\end{array}$ & $\begin{array}{c}0.11 \\
{[0.09,0.14]}\end{array}$ \\
\hline $\begin{array}{l}\text { Consump. } \\
\text { growth }\end{array}$ & $\begin{array}{c}0.38 \\
{[0.22,0.56]}\end{array}$ & $\begin{array}{c}0.13 \\
{[0.06,0.21]}\end{array}$ & $\begin{array}{c}0.07 \\
{[0.02,0.11]}\end{array}$ & $\begin{array}{c}0.16 \\
{[0.11,0.21]}\end{array}$ & $\begin{array}{c}0.11 \\
{[0.07,0.16]}\end{array}$ & $\begin{array}{c}0.12 \\
{[0.03,0.21]}\end{array}$ & $\begin{array}{c}0.01 \\
{[0.00,0.02]}\end{array}$ \\
\hline $\begin{array}{l}\text { Investmt. } \\
\text { growth }\end{array}$ & $\begin{array}{c}0.02 \\
{[0.00,0.03]}\end{array}$ & $\begin{array}{c}0.01 \\
{[0.00,0.02]}\end{array}$ & $\begin{array}{c}0.03 \\
{[0.01,0.04]}\end{array}$ & $\begin{array}{c}0.01 \\
{[0.01,0.02]}\end{array}$ & $\begin{array}{c}0.03 \\
{[0.02,0.04]}\end{array}$ & $\begin{array}{c}0.91 \\
{[0.87,0.95]}\end{array}$ & $\begin{array}{c}0.00 \\
{[0.00,0.00]}\end{array}$ \\
\hline $\begin{array}{l}\text { Real wage } \\
\text { growth }\end{array}$ & $\begin{array}{c}0.01 \\
{[0.00,0.01]}\end{array}$ & $\begin{array}{c}0.60 \\
{[0.51,0.70]}\end{array}$ & $\begin{array}{c}0.28 \\
{[0.20,0.39]}\end{array}$ & $\begin{array}{c}0.01 \\
{[0.00,0.01]}\end{array}$ & $\begin{array}{c}0.06 \\
{[0.04,0.09]}\end{array}$ & $\begin{array}{c}0.04 \\
{[0.02,0.07]}\end{array}$ & $\begin{array}{c}0.00 \\
{[0.00,0.00]}\end{array}$ \\
\hline $\begin{array}{l}\text { Total hours } \\
\text { growth }\end{array}$ & $\begin{array}{c}\text { s } 0.03 \\
{[0.01,0.07]}\end{array}$ & $\begin{array}{c}0.32 \\
{[0.16,0.47]}\end{array}$ & $\begin{array}{c}0.18 \\
{[0.07,0.27]}\end{array}$ & $\begin{array}{c}0.04 \\
{[0.02,0.06]}\end{array}$ & $\begin{array}{c}0.03 \\
{[0.02,0.05]}\end{array}$ & $\begin{array}{c}0.31 \\
{[0.17,0.44]}\end{array}$ & $\begin{array}{c}0.09 \\
{[0.03,0.13]}\end{array}$ \\
\hline Inflation & $\begin{array}{c}0.01 \\
{[0.00,0.03]}\end{array}$ & $\begin{array}{c}0.40 \\
{[0.25,0.55]}\end{array}$ & $\begin{array}{c}0.38 \\
{[0.22,0.52]}\end{array}$ & $\begin{array}{c}0.04 \\
{[0.02,0.07]}\end{array}$ & $\begin{array}{c}0.06 \\
{[0.04,0.09]}\end{array}$ & $\begin{array}{c}0.09 \\
{[0.02,0.16]}\end{array}$ & $\begin{array}{c}0.01 \\
{[0.00,0.01]}\end{array}$ \\
\hline $\begin{array}{l}\text { Interest } \\
\text { rate }\end{array}$ & $\begin{array}{c}0.08 \\
{[0.01,0.17]}\end{array}$ & $\begin{array}{c}0.17 \\
{[0.08,0.26]}\end{array}$ & $\begin{array}{c}0.09 \\
{[0.03,0.15]}\end{array}$ & $\begin{array}{c}0.12 \\
{[0.07,0.17]}\end{array}$ & $\begin{array}{c}0.09 \\
{[0.06,0.13]}\end{array}$ & $\begin{array}{c}0.43 \\
{[0.27,0.62]}\end{array}$ & $\begin{array}{c}0.01 \\
{[0.01,0.02]}\end{array}$ \\
\hline
\end{tabular}

Notes: Each column corresponds to the contribution of a particular structural shock to the variance of observables. The values in square brackets are 90 percent Bayesian confidence intervals.

The dominant role of IST shocks highlighted by Justiniano et al. (2010) is replicated in this version of the model as 55 percent of the variance of output growth is accounted for by IST shocks. Risk premium, neutral technology and government spending shocks jointly make up another 30 percent of the variance of output growth. IST shocks also account for almost all (91 percent) of the variance of investment growth and a large part of the variance of the nominal interest rate (43 percent).

In this model IST shocks are particularly important in capturing the decline in output that occurred during the Great Recession. Figure 4.1 shows the path of output growth when the model is driven solely by IST shocks. Here, IST shocks account for over half of the drop in output growth during the last recession. The premise of this chapter is that this result is not robust to the introduction of financial frictions in the form of borrowing constraints. 
Figure 4.1: Role of IST shock in the Great Recession in the model without borrowing constraints

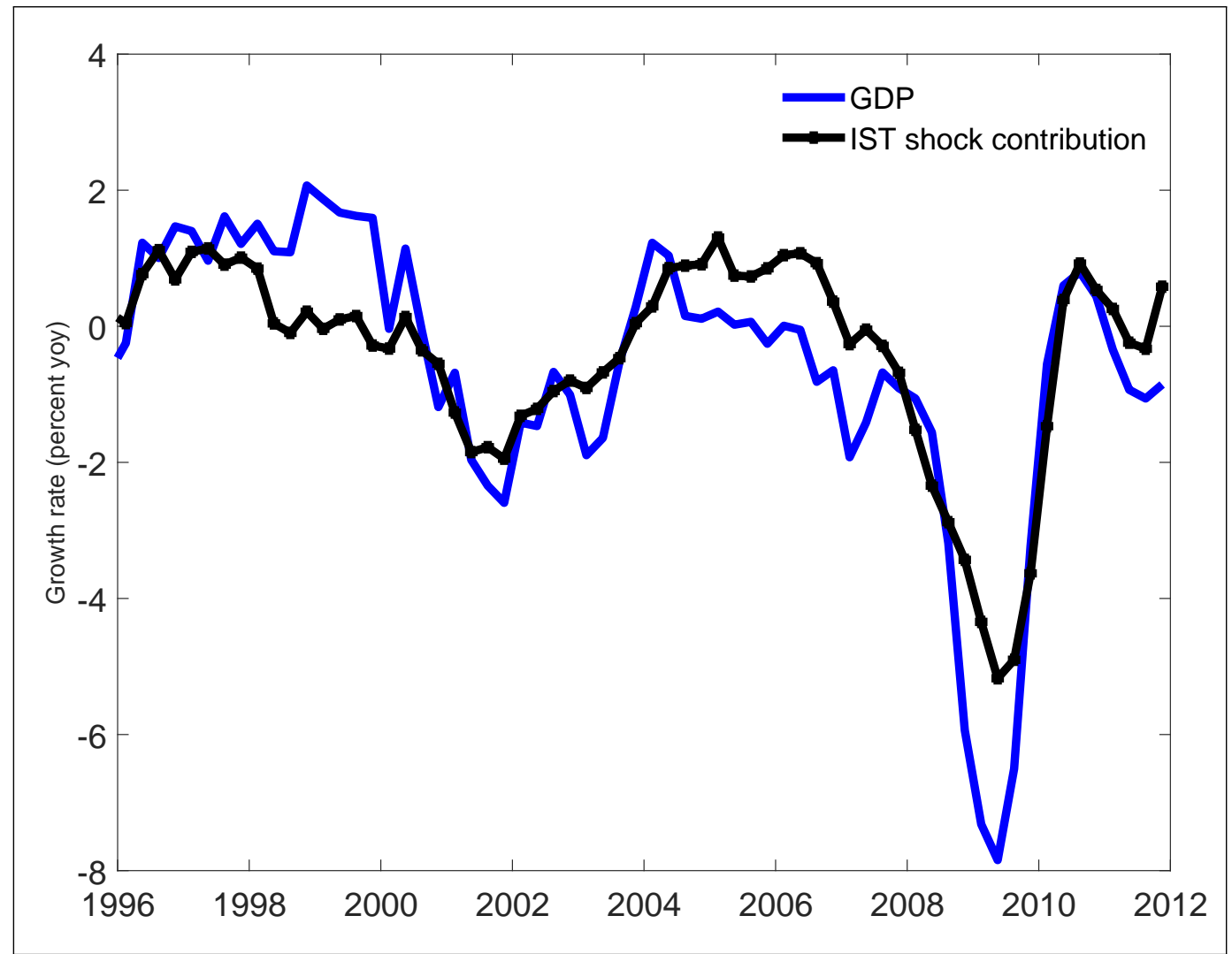

Notes: The solid line labelled Data shows the year-on-year growth rate of GDP. The solidcrossed line, labelled IST shock contribution, shows the growth rate of GDP that would have occurred if only the estimated IST shocks assume non-zero values. The estimated shocks are obtained via the Kalman smoother on the estimated posterior mean of the Smets-Wouters model with no borrowing constraints. 
Table 4.5: Variance decomposition of model with borrowing constraints:

\begin{tabular}{|c|c|c|c|c|c|c|c|}
\hline & $\begin{array}{c}\text { Risk } \\
\text { premium }\end{array}$ & $\begin{array}{c}\text { Wage } \\
\text { markup }\end{array}$ & $\begin{array}{l}\text { Price } \\
\text { markup }\end{array}$ & $\begin{array}{c}\text { Monetary } \\
\text { policy }\end{array}$ & $\begin{array}{c}\text { Neutral } \\
\text { Technology }\end{array}$ & IST & Govt. \\
\hline $\begin{array}{l}\text { Output } \\
\text { growth }\end{array}$ & $\begin{array}{c}0.47 \\
{[0.43,0.53]}\end{array}$ & $\begin{array}{c}0.05 \\
{[0.03,0.07]}\end{array}$ & $\begin{array}{c}0.04 \\
{[0.02,0.06]}\end{array}$ & $\begin{array}{c}0.20 \\
{[0.18,0.23]}\end{array}$ & $\begin{array}{c}0.06 \\
{[0.03,0.09]}\end{array}$ & $\begin{array}{c}0.04 \\
{[0.01,0.06]}\end{array}$ & $\begin{array}{c}0.14 \\
{[0.12,0.15]}\end{array}$ \\
\hline $\begin{array}{l}\text { Consump. } \\
\text { growth }\end{array}$ & $\begin{array}{c}0.46 \\
{[0.40,0.52]}\end{array}$ & $\begin{array}{c}0.05 \\
{[0.03,0.08]}\end{array}$ & $\begin{array}{c}0.04 \\
{[0.02,0.06]}\end{array}$ & $\begin{array}{c}0.20 \\
{[0.16,0.23]}\end{array}$ & $\begin{array}{c}0.05 \\
{[0.02,0.06]}\end{array}$ & $\begin{array}{c}0.20 \\
{[0.14,0.25]}\end{array}$ & $\begin{array}{c}0.00 \\
{[0.00,0.01]}\end{array}$ \\
\hline $\begin{array}{l}\text { Investmt. } \\
\text { growth }\end{array}$ & $\begin{array}{c}0.39 \\
{[0.31,0.48]}\end{array}$ & $\begin{array}{c}0.02 \\
{[0.01,0.04]}\end{array}$ & $\begin{array}{c}0.04 \\
{[0.01,0.06]}\end{array}$ & $\begin{array}{c}0.17 \\
{[0.13,0.21]}\end{array}$ & $\begin{array}{c}0.01 \\
{[0.00,0.02]}\end{array}$ & $\begin{array}{c}0.37 \\
{[0.23,0.50]}\end{array}$ & $\begin{array}{c}0.00 \\
{[0.00,0.00]}\end{array}$ \\
\hline $\begin{array}{l}\text { Real wage } \\
\text { growth }\end{array}$ & $\begin{array}{c}0.01 \\
{[0.00,0.02]}\end{array}$ & $\begin{array}{c}0.81 \\
{[0.76,0.87]}\end{array}$ & $\begin{array}{c}0.15 \\
{[0.11,0.19]}\end{array}$ & $\begin{array}{c}0.00 \\
{[0.00,0.01]}\end{array}$ & $\begin{array}{c}0.02 \\
{[0.01,0.03]}\end{array}$ & $\begin{array}{c}0.00 \\
{[0.00,0.00]}\end{array}$ & $\begin{array}{c}0.00 \\
{[0.00,0.00]}\end{array}$ \\
\hline $\begin{array}{l}\text { Total hours } \\
\text { growth }\end{array}$ & $\begin{array}{l}\mathrm{s} \quad 0.23 \\
{[0.12,0.35]}\end{array}$ & $\begin{array}{c}0.32 \\
{[0.14,0.49]}\end{array}$ & $\begin{array}{c}0.06 \\
{[0.01,0.10]}\end{array}$ & $\begin{array}{c}0.10 \\
{[0.04,0.14]}\end{array}$ & $\begin{array}{c}0.06 \\
{[0.02,0.09]}\end{array}$ & $\begin{array}{c}0.01 \\
{[0.00,0.02]}\end{array}$ & $\begin{array}{c}0.23 \\
{[0.10,0.36]}\end{array}$ \\
\hline Inflation & $\begin{array}{c}0.02 \\
{[0.00,0.05]}\end{array}$ & $\begin{array}{c}0.43 \\
{[0.28,0.58]}\end{array}$ & $\begin{array}{c}0.50 \\
{[0.35,0.65]}\end{array}$ & $\begin{array}{c}0.01 \\
{[0.00,0.01]}\end{array}$ & $\begin{array}{c}0.04 \\
{[0.01,0.07]}\end{array}$ & $\begin{array}{c}0.00 \\
{[0.00,0.00]}\end{array}$ & $\begin{array}{c}0.00 \\
{[0.00,0.00]}\end{array}$ \\
\hline $\begin{array}{l}\text { Interest } \\
\text { rate }\end{array}$ & $\begin{array}{c}0.40 \\
{[0.25,0.55]}\end{array}$ & $\begin{array}{c}0.25 \\
{[0.14,0.37]}\end{array}$ & $\begin{array}{c}0.14 \\
{[0.07,0.22]}\end{array}$ & $\begin{array}{c}0.10 \\
{[0.06,0.13]}\end{array}$ & $\begin{array}{c}0.11 \\
{[0.07,0.15]}\end{array}$ & $\begin{array}{c}0.00 \\
{[0.00,0.01]}\end{array}$ & $\begin{array}{c}0.00 \\
{[0.00,0.01]}\end{array}$ \\
\hline
\end{tabular}

Notes: Each column corresponds to the contribution of a particular structural shock to the variance of observables. The values in square brackets are 90 percent Bayesian confidence intervals.

Introducing a borrowing constraint on entrepreneurs affects the transmission mechanism of IST shocks and thus their relative contribution to the volatility of GDP. The variance decomposition of the observables in Table 4.5 illustrates that in the model with borrowing constraints, the role of IST shocks is greatly reduced. Apart from consumption and investment, IST shocks account for less than 5 percent of the volatility of the observable variables. Their contribution to the dynamics of investment remains significant but almost two thirds less than in the model without the borrowing constraint. The higher share of IST shocks in the volatility of consumption reflects these shocks' role in the dynamics of entrepreneurial consumption. In section 4.4.1, we examine this channel in more detail.

In the model with borrowing constraint, the main driver of business cycle fluctuations appears to be the risk premium, contributing between 39 per- 
cent and 47 percent to the variance of the components of GDP. Adding a borrowing constraint also increases the share of risk premium shocks in the variance of total hours and nominal interest rates.

Given its importance in shaping business cycle dynamics, we now examine how the risk premium shock evolves over the business cycle. Figure 4.2 plots the posterior mean of our estimated risk premium shock and the NBER recession dates which start at the peak of a business cycle and end at the trough. The sample includes every recession from the late 1950s onwards. There is a striking conformity of the risk premium shock with these recessions. At the beginning of each recession the estimated risk premium shock rises sharply, implying that the effective interest rate in the model is highly countercyclical. Moreover, the risk premium and the effective interest rate start to rise before the peak of the boom, in almost every recession in our sample.

The increase in our measure of the risk premium shock is most pronounced during the last recession. Figure 4.3 illustrates the role of risk premium shocks over the last decade and a half by simulating the path of output assuming that the model is only driven by the estimated risk premium shock. Most of the drop in output growth in the last recession is due to the variation in the risk premium shock. This is in line with the observation that the last recession was driven by sharp disruptions in the financial system resulting in higher interest rate spreads.

\subsubsection{IST shocks and collateral constraints}

The following two sections flesh out the intuition behind our results starting with the role of IST shocks. In a real business cycle type model, investment rises but consumption falls following a positive IST shock (see for example Barro and King 1984). A shock that increases the marginal efficiency of investment raises the incentive to invest by more than can be accommodated by an increase in labour effort. As a result, investment can only increase sufficiently if consumption falls. This GDP-consumption co-movement puzzle precludes IST shocks from being a key driver of the business cycle in this type of model. Justiniano et al. (2010) show how this co-movement puzzle can be 
Figure 4.2: The risk-premium shock and NBER recession intervals in the model with borrowing constraints

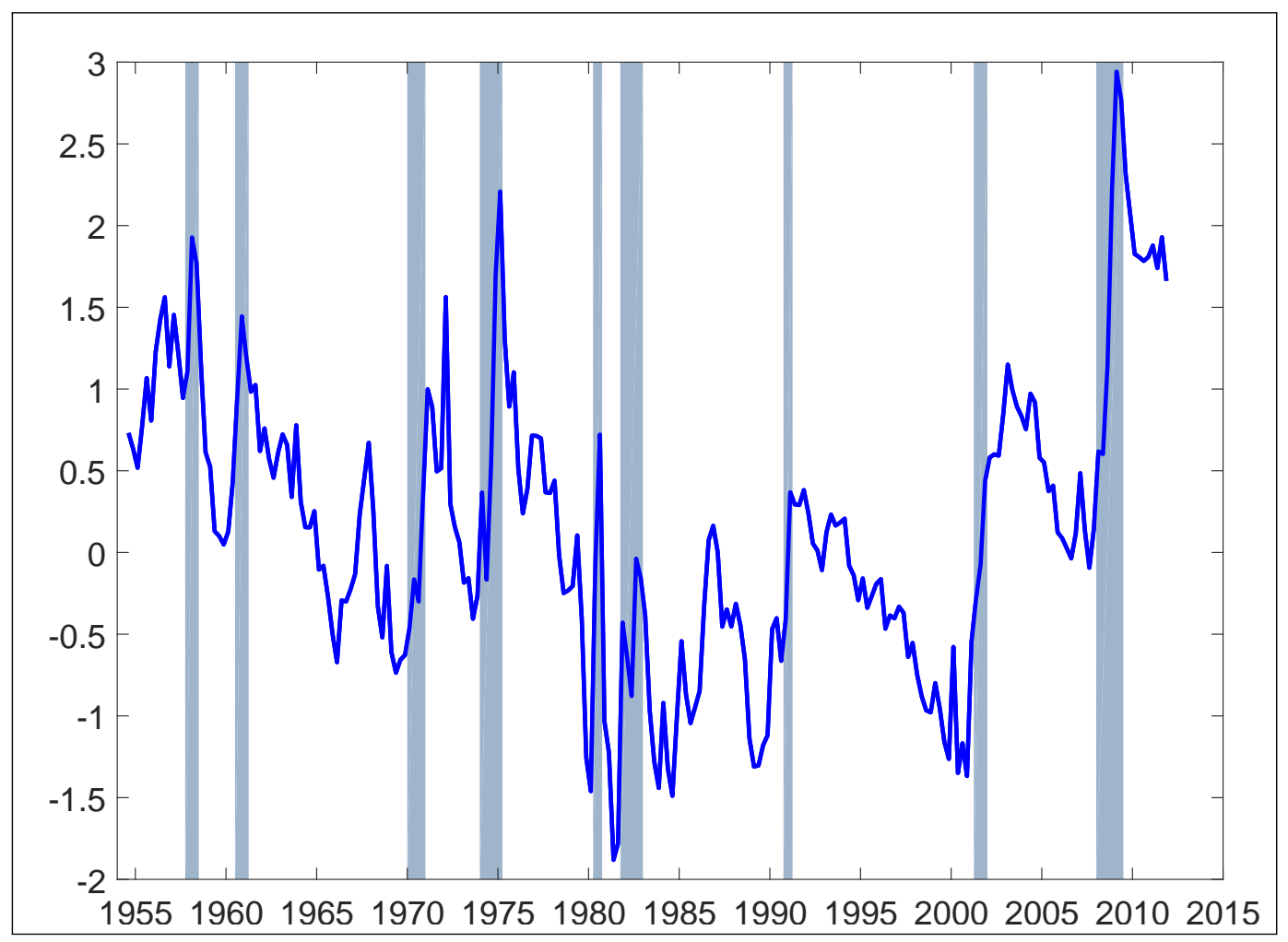

Notes: The solid line shows the estimated risk premium shocks for the model with the borrowing constraint. The estimated shocks are obtained via the Kalman smoother on the estimated posterior mean. The shaded areas correspond to the NBER recession intervals.

overcome through a combination of nominal and real rigidities plus variable capital utilization. ${ }^{9}$ As a result, their model is able to generate a dominant role for IST shocks over the business cycle, although these shocks have a limited role in accounting for consumption movements. All the features that account for the co-movement puzzle in Justiniano et al. are also present in our model, in addition to the binding borrowing constraint on entrepreneurs.

Figure 4.4 shows the impulse response functions following an IST shock

\footnotetext{
${ }^{9}$ Greenwood et al. (2000) and more recently Furlanetto and Seneca (2014) and Khan and Tsoukalas (2011), discuss a number of ways in which the positive co-movement of consumption and investment can be derived, including non-separable preferences, habit persistence and factor immobility, intratemporal adjustment costs on investment, and intermediate inputs.
} 
Figure 4.3: Role of Risk Premium shock in the Great Recession in the model with borrowing constraints

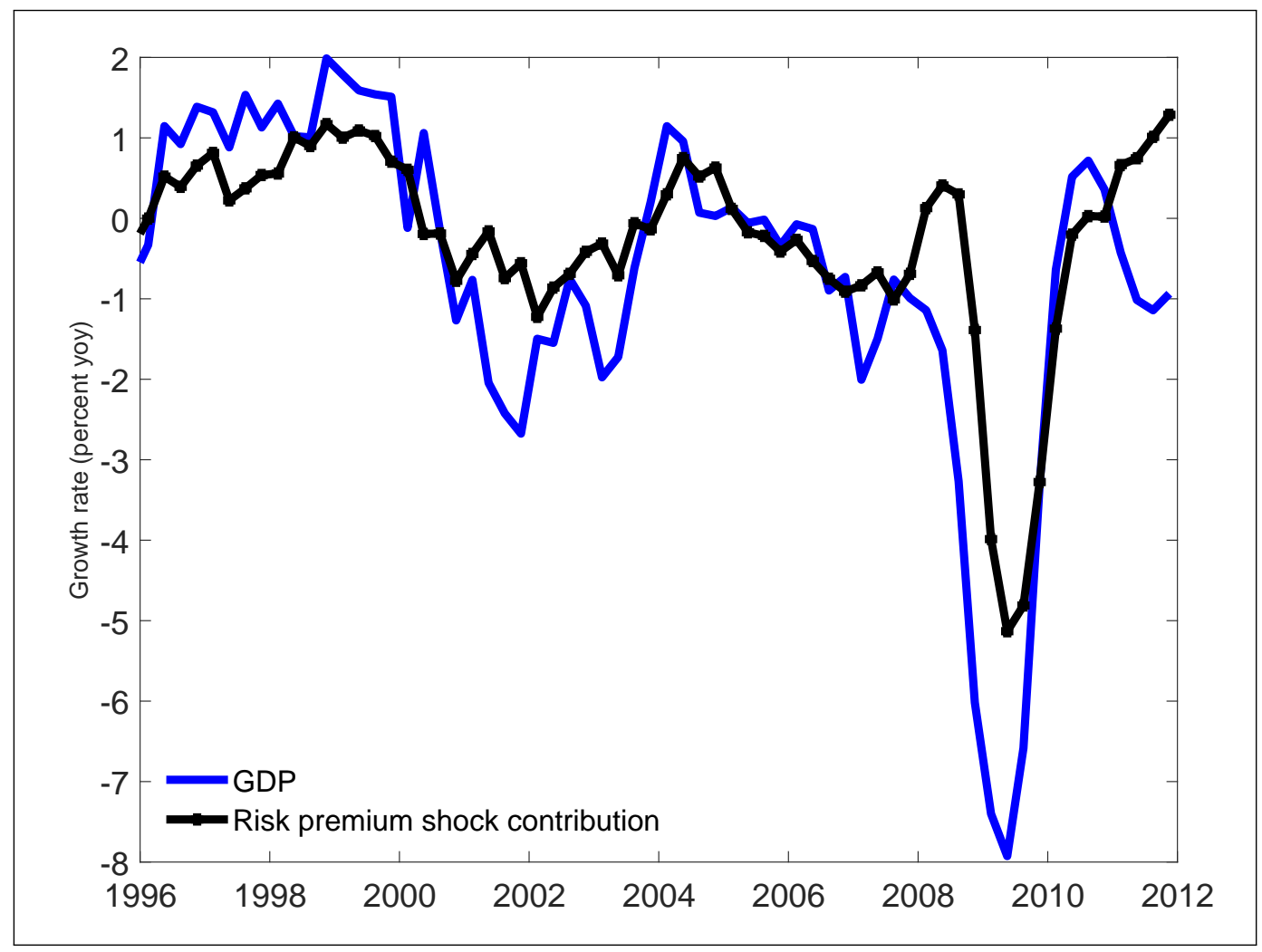

Notes: The solid line labelled Data shows the year-on-year growth rate of GDP. The solidcrossed line, labelled Risk premium shock contribution, shows the growth rate of GDP that would have occurred if only the estimated Risk premium shocks assume non-zero values. The estimated shocks are obtained via the Kalman smoother on the estimated posterior mean of the model with the borrowing constraint. 
in our estimated model. The solid lines show the median response and the shaded areas the 90 percent confidence intervals. As Figure 4.4 makes clear, there is no co-movement puzzle between GDP and household consumption. However, aggregate consumption declines because of a sharp adjustment to entrepreneurs' consumption in the wake of a positive IST shock. A positive IST shock reduces the value of Tobin's q (this is true even in a simple RBC model without adjustment costs where $\left.1=Q_{t} \varepsilon_{\mu, t}\right)$ and thus the value of the capital stock used for collateral. The decline in the value of collateral, other things equal, reduces firms' ability to borrow just when the demand for borrowing coming from investment is high. As a result, investment is reduced relative to the case without borrowing constraints, and entrepreneurs' consumption falls. In terms of the entrepreneurs' Euler equation, (4.9), a decline in Tobin's q tightens the borrowing constraint causing $\lambda_{t}^{e}$ to rise, which, other things equal, causes entrepreneurial consumption to fall. In the estimated model entrepreneurs' consumption falls by enough to lead to a decline in aggregate consumption.

\subsubsection{Risk premium shocks and borrowing constraints}

The volatilities of observed variables ultimately stem from some underlying structural shocks. In the context of the model with borrowing constraints, the risk premium shocks supplant investment shocks. The same channel that reduces the impact of IST shocks contributes to the increase in the importance of risk premium shocks. Figure 4.5 shows the transmission mechanism of a risk premium shock. A negative risk premium shock lowers the effective interest rates faced by household and entrepreneurs. This results in higher consumption and output, generating an increased demand for investment and a higher price of capital. From the perspective of entrepreneurs, even in the absence of any borrowing constraint, the lower cost of servicing their debt allows them to increase both their consumption and capital purchases. The additional asset price channel (higher Tobin's q) implies that they also face a looser borrowing constraint (both $\lambda_{t}^{e}$ and $\varepsilon_{c, t}$ decline in equation (4.9) causing entrepreneurial consumption to rise). This engenders an amplification of the impact of risk premium shocks for both consumption and investment. 
Figure 4.4: Impulse response to an IST shock in the model with borrowing constraints

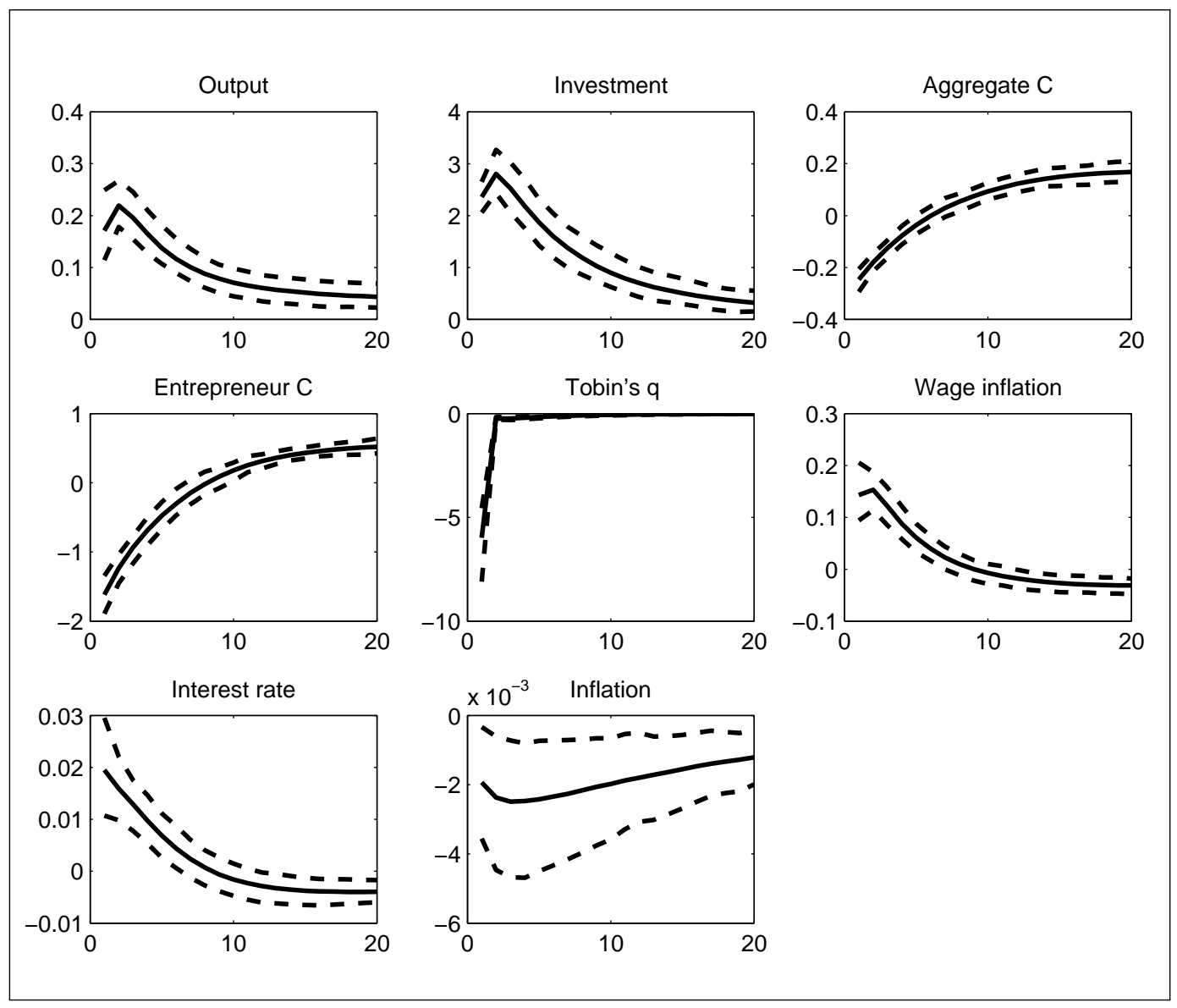

Notes: The solid lines are impulse response functions to a one standard deviation shock. The solid line is the posterior median, the shaded areas correspond to the 90 percent Bayesian confidence intervals. Responses are measured as the percentage deviations from trend except for inflation and interest rates, which are measured as the percentage point deviation from steady state values. 
Figure 4.5: Impulse response to a risk premium shock in the model with borrowing constraints

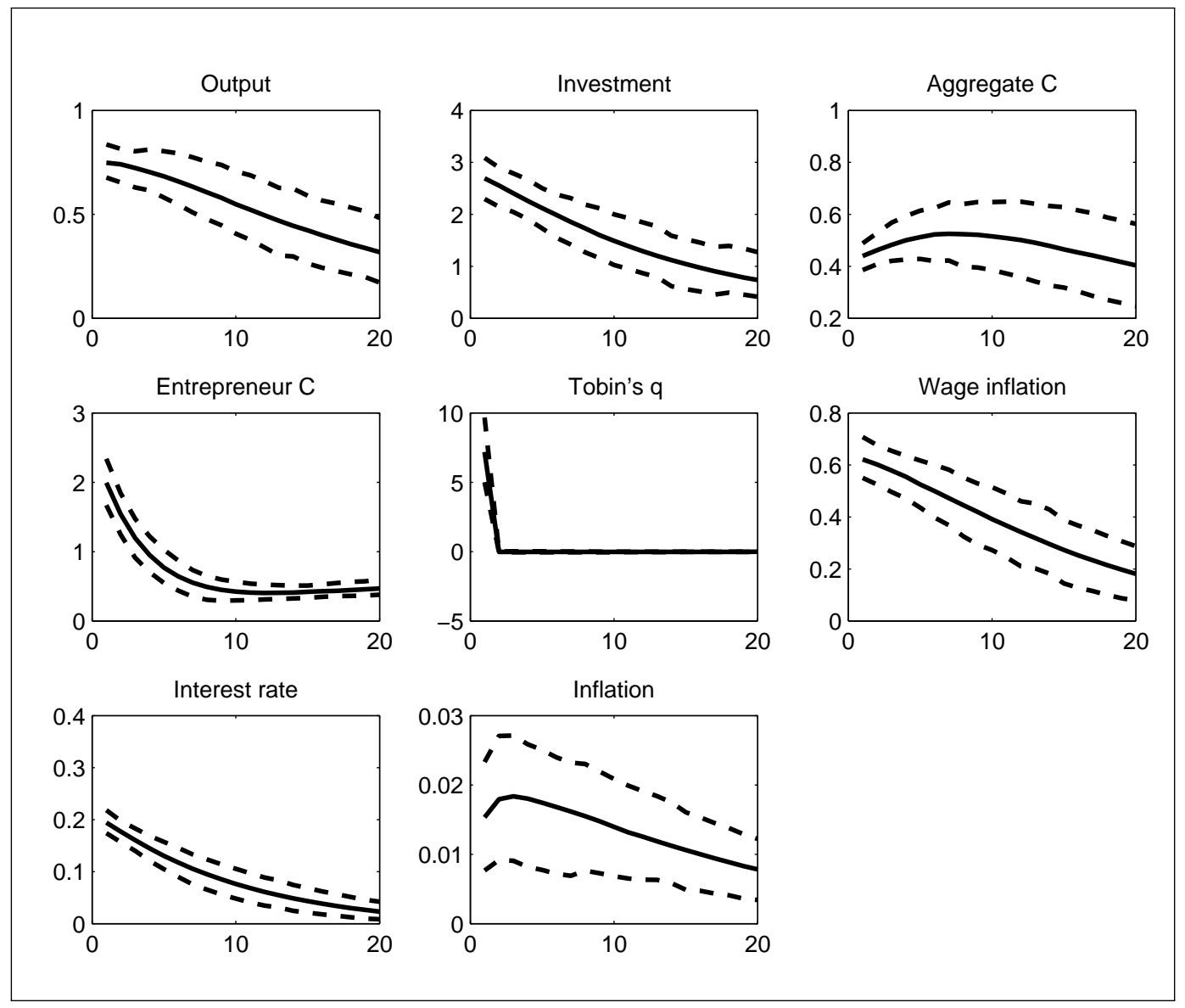

Notes: The solid lines are impulse response functions to a one standard deviation shock. The solid line is the posterior median, the shaded areas correspond to the 90 percent Bayesian confidence intervals. Responses are measured as the percentage deviations from trend except for inflation and interest rates, which are measured as the percentage point deviation from steady state values. 
As the response of interest rates and inflation are positive, our model generates positive co-movement between macroeconomic aggregates following a risk premium shock.

Our analysis shows that the introduction of the borrowing constraint alters the transmission mechanisms of both IST and risk premium shocks. The borrowing constraint attenuates the expansionary effects of IST shocks on output, whereas the impact of risk premium shocks is amplified.

\subsection{Discussion}

Our analysis suggests that risk premium shocks, or shocks to the effective interest rate faced by households and firms, are the main driver of the business cycle. This result is attributable to the role played by simple financial frictions in the form of borrowing constraints. An expansionary risk premium shock loosens the borrowing constraint faced by entrepreneurs and thus reduces the cost of transforming household savings into productive capital.

Justiniano et al. (2011), in a model without explicit financial frictions, attribute this role to IST shocks. A positive IST shock raises the marginal efficiency of investment and thus the rate with which household savings are transformed into productive capital. As a supply type shock, a positive IST shock also yields a decline in the price of capital. In the presence of borrowing constraints, the counter-cyclical asset price movement tends to tighten the borrowing constraint and this mechanism reduces the contribution of IST shocks.

Christensen and Dib (2008), and more recently Merola (2015), compare models with and without a financial accelerator mechanism, where firms' net worth affects the 'external finance premium' and thus the firms' costs of borrowing. Even though there are significant differences between their approach and ours (in terms of sample period, model and estimation technique), they too find that the role of IST shocks in the forecast variance of GDP diminishes in the presence of financial frictions, albeit to a much lesser extent. The financial friction in Christensen and Dib (2008) has a mild effect on the transmission mechanism quantitatively, but the dynamics are qualitatively unchanged. In our model, financial frictions reverse the short term impact of 
IST shocks on aggregate consumption, and thus have both quantitative and qualitative effects on the response of output.

A number of recent papers in the literature view the financial sector as a source of shocks driving the business cycle. For example, Nolan and Thoenissen (2009) show in a Bernanke et al. (1999) type model that shocks to entrepreneurial net worth play a key role in the dynamics of GDP. Christiano et al. (2012) estimates a modified financial accelerator model where the volatility of idiosyncratic shocks in the financial accelerator mechanism is treated as a stochastic process. This risk shock is shown to account for a large proportion of the volatility of GDP when the model is estimated on financial data. As in our analysis, the contribution of investment shocks declines, once risk shocks are introduced. Hirakata et al. (2011) also introduce shocks to financial intermediation in a BGG-type model and find shocks to financial intermediation play an important role in the dynamics of investment, in particular accounting for the collapse of investment during the financial crisis. Jermann and Quadrini (2012) investigate the importance of shocks originating in the financial sector when firms face borrowing constraints. As in the previous literature, these financial shocks are found to be quantitatively important.

In relation to this literature, our results highlight the importance of risk premium shocks. Although this type of shock is present in canonical DSGE models such as Smets and Wouters (2007), its role as a driver of the business cycle only comes to the fore once we introduce the borrowing constraint. In contrast to Christiano et al. (2012), our risk premium shock becomes important in the presence of financial frictions, without using financial data in the estimation of the model.

Our results share some similarities with Iacoviello (2005), who points out that, in a model with real estate investments, the effects of borrowing constraints on the amplification of shocks depend on the response of asset prices and consumer price inflation. In his framework, where household debt is denominated in nominal terms, shocks that generate a negative correlation between inflation and output (such as supply shocks), are decelerated while the impact of demand shocks are amplified. Our contribution extends this channel to the case of investment shocks. 


\subsection{Robustness over the sample}

This section analyzes the robustness of our results to alternative sample periods. Our baseline estimation period runs from 1954Q3 through to 2011Q4 and therefore encompasses the estimation periods of, amongst others, Justiniano et al. (2010, 2011) and Smets and Wouters (2007). This estimation period spans two recent episodes that have the potential to affect our results: the Great Moderation, from the Volcker disinflation up to the Financial Crisis; and the post 2009Q1 period where the zero lower bound for the federal funds rate becomes binding. To check for the robustness of our results, we re-estimate the model for the 1984Q1 to 2009Q1 period.

Tables 4.6 and 4.7 present the estimation results and variance decomposition for this alternative sample. Our parameter estimates are broadly consistent with those obtained in the baseline estimation. The main changes relate to the dynamics of investment. As the volatility of investment is lower during that period, the model requires a higher investment adjustment cost parameter as well as a lower volatility for the IST shock. The posterior mean of the investment adjustment cost parameter is about three times larger, while the posterior mean of the standard deviation of the IST shock is halved.

Table 4.6: Estimation results for parameters and shock processes of model with borrowing constraints: 1984Q1 2009Q1

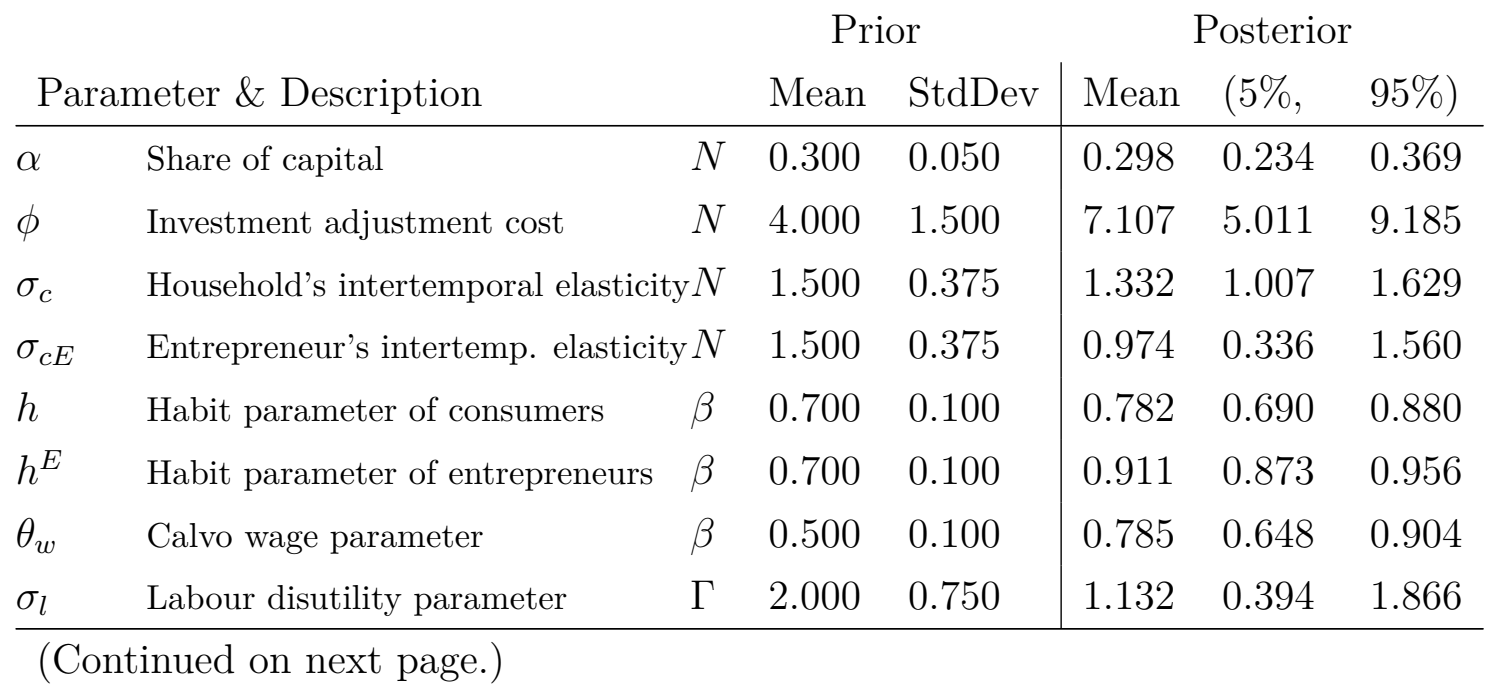




\begin{tabular}{|c|c|c|c|c|c|c|c|}
\hline \multirow{2}{*}{\multicolumn{3}{|c|}{ Parameter \& Description }} & \multicolumn{2}{|c|}{ Prior } & \multicolumn{3}{|c|}{ Posterior } \\
\hline & & & Mean & StdDev & Mean & $(5 \%$ & $95 \%)$ \\
\hline$\overline{\theta_{p}}$ & Calvo price parameter & $\beta$ & 0.500 & 0.100 & 0.780 & 0.687 & 0.899 \\
\hline$\xi$ & Capacity utilization parameter & $\beta$ & 0.500 & 0.150 & 0.667 & 0.516 & 0.819 \\
\hline$\phi_{p}$ & Markup (goods) & $N$ & 1.250 & 0.125 & 1.329 & 1.201 & 1.467 \\
\hline$\delta_{w}$ & Wage indexation & $\beta$ & 0.500 & 0.150 & 0.384 & 0.162 & 0.602 \\
\hline$\delta_{p}$ & Price indexation & $\beta$ & 0.500 & 0.150 & 0.268 & 0.104 & 0.442 \\
\hline$\phi_{\pi}$ & Taylor rule inflation & $N$ & 1.500 & 0.250 & 2.194 & 1.804 & 2.580 \\
\hline$\phi_{r}$ & Taylor rule lagged interest rate & $\beta$ & 0.750 & 0.100 & 0.825 & 0.786 & 0.866 \\
\hline$\phi_{x}$ & Taylor rule output gap & $N$ & 0.125 & 0.050 & 0.064 & 0.019 & 0.107 \\
\hline$\phi_{\Delta x}$ & Taylor rule output gap growth rate & $N$ & 0.125 & 0.050 & 0.154 & 0.115 & 0.193 \\
\hline $\bar{\pi}$ & Steady state inflation & $\Gamma$ & 0.625 & 0.100 & 0.773 & 0.683 & 0.856 \\
\hline$\frac{100(1-\beta)}{\beta}$ & Discount rate (percent) & $\Gamma$ & 0.250 & 0.100 & 0.248 & 0.099 & 0.390 \\
\hline$l t v$ & Loan to value ratio & $\beta$ & 0.500 & 0.150 & 0.572 & 0.328 & 0.811 \\
\hline$\tilde{\beta}$ & Entrepreneurs discount less h'hlds & $\Gamma$ & 1.000 & 0.500 & 0.989 & 0.235 & 1.690 \\
\hline lss & Log steady state hours & $N$ & 0.000 & 2.000 & 0.313 & -1.601 & 2.240 \\
\hline$\gamma$ & Steady state growth rate (percent) & $N$ & 0.400 & 0.100 & 0.362 & 0.269 & 0.447 \\
\hline
\end{tabular}

(Continued on next page.) 


\begin{tabular}{|c|c|c|c|c|c|c|c|}
\hline \multirow{2}{*}{\multicolumn{3}{|c|}{ Parameter \& Description }} & \multicolumn{2}{|c|}{ Prior } & \multicolumn{3}{|c|}{ Posterior } \\
\hline & & & \multirow{2}{*}{$\begin{array}{l}\text { Mean } \\
0.500\end{array}$} & \multirow{2}{*}{$\frac{\text { StdDev }}{0.200}$} & \multirow{2}{*}{$\frac{\text { Mean }}{0.959}$} & \multirow{2}{*}{$\frac{(5 \%,}{0.932}$} & \multirow{2}{*}{$\frac{95 \%)}{0.986}$} \\
\hline$\rho_{a}$ & AR parameter technology & $\beta$ & & & & & \\
\hline$\rho_{c}$ & AR parameter risk premium & $\beta$ & 0.500 & 0.200 & 0.632 & 0.380 & 0.870 \\
\hline$\rho_{g}$ & AR parameter exog. Dd. & $\beta$ & 0.500 & 0.200 & 0.952 & 0.911 & 0.991 \\
\hline$\rho_{i}$ & AR parameter investment & $\beta$ & 0.500 & 0.200 & 0.389 & 0.276 & 0.503 \\
\hline$\rho_{r}$ & AR parameter interest rate & $\beta$ & 0.500 & 0.200 & 0.278 & 0.149 & 0.403 \\
\hline$\rho_{p}$ & AR parameter price markup & $\beta$ & 0.500 & 0.200 & 0.900 & 0.820 & 0.979 \\
\hline$\rho_{w}$ & AR parameter wage markup & $\beta$ & 0.500 & 0.200 & 0.861 & 0.760 & 0.985 \\
\hline$\rho_{e p}$ & MA parameter price markup & $\beta$ & 0.500 & 0.200 & 0.745 & 0.586 & 0.943 \\
\hline$\rho_{e w}$ & MA parameter wage markup & $\beta$ & 0.500 & 0.200 & 0.800 & 0.680 & 0.940 \\
\hline$\rho_{g a}$ & Effect of tech shock on exog. Dd. & $N$ & 0.500 & 0.200 & 0.285 & 0.165 & 0.400 \\
\hline$\sigma_{c}$ & Std dev. of risk premium shock & $\Gamma^{-1}$ & 0.100 & 2.000 & 0.355 & 0.205 & 0.513 \\
\hline$\sigma_{w}$ & Std dev. of wage markup shock & $\Gamma^{-1}$ & 0.100 & 2.000 & 0.323 & 0.267 & 0.378 \\
\hline$\sigma_{p}$ & Std dev. of price markup shock & $\Gamma^{-1}$ & 0.100 & 2.000 & 0.109 & 0.076 & 0.135 \\
\hline$\sigma_{r}$ & Std dev. of interest rate shock & $\Gamma^{-1}$ & 0.100 & 2.000 & 0.126 & 0.108 & 0.145 \\
\hline$\sigma_{a}$ & Std dev. of technology shock & $\Gamma^{-1}$ & 0.100 & 2.000 & 0.450 & 0.385 & 0.502 \\
\hline$\sigma_{i}$ & Std dev. of investment shock & $\Gamma^{-1}$ & 0.100 & 2.000 & 1.075 & 0.816 & 1.305 \\
\hline$\sigma_{g}$ & Std dev. of exog. demand shock & $\Gamma^{-1}$ & 0.100 & 2.000 & 0.272 & 0.239 & 0.307 \\
\hline
\end{tabular}

Notes: The prior for a parameter is a Normal $(N)$, Beta $(\beta)$, Gamma $(\Gamma)$, or inverseGamma $\left(\Gamma^{-1}\right)$ distribution. Columns 4 and 5 indicate the mean and standard deviation of the prior distribution, and the final three columns report the posterior mean and lower and upper limits of 90 percent Bayesian confidence intervals from the posterior distribution.

The main conclusion regarding the drivers of the business cycle remains unchanged. In the context of the model with borrowing constraints, the risk premium shock remains the dominant driver of the volatility in the components of GDP. 
Table 4.7: Variance decomposition of model with borrowing constraints: 1984Q1 - 2009Q1

\begin{tabular}{|c|c|c|c|c|c|c|c|}
\hline & $\begin{array}{c}\text { Risk } \\
\text { premium }\end{array}$ & $\begin{array}{c}\text { Wage } \\
\text { markup }\end{array}$ & $\begin{array}{c}\text { Price } \\
\text { markup }\end{array}$ & $\begin{array}{c}\text { Monetary } \\
\text { policy }\end{array}$ & $\begin{array}{c}\text { Neutral } \\
\text { Technology }\end{array}$ & IST & Govt. \\
\hline Output & 0.40 & 0.09 & 0.07 & 0.20 & 0.06 & 0.07 & 0.11 \\
\hline growth & {$[0.18,0.61]$} & {$[0.04,0.13]$} & {$[0.00,0.13]$} & {$[0.11,0.28]$} & {$[0.02,0.11]$} & {$[0.02,0.13]$} & {$[0.06,0.15]$} \\
\hline Consump. & 0.48 & 0.08 & 0.06 & 0.25 & 0.04 & 0.09 & 0.00 \\
\hline growth & {$[0.25,0.70]$} & {$[0.03,0.13]$} & {$[0.00,0.12]$} & {$[0.12,0.36]$} & {$[0.01,0.06]$} & {$[0.01,0.16]$} & {$[0.00,0.00]$} \\
\hline Invest. & 0.24 & 0.04 & 0.04 & 0.12 & 0.01 & 0.55 & 0.00 \\
\hline growth & {$[0.04,0.43]$} & {$[0.01,0.07]$} & {$[0.00,0.08]$} & {$[0.05,0.18]$} & {$[0.00,0.02]$} & {$[0.28,0.78]$} & {$[0.00,0.00]$} \\
\hline Real wage & e $\quad 0.01$ & 0.85 & 0.11 & 0.01 & 0.02 & 0.01 & 0.00 \\
\hline growth & {$[0.00,0.03]$} & {$[0.74,0.94]$} & {$[0.01,0.18]$} & {$[0.00,0.02]$} & {$[0.00,0.03]$} & {$[0.00,0.02]$} & {$[0.00,0.00]$} \\
\hline Total & 0.18 & 0.30 & 0.19 & 0.13 & 0.07 & 0.05 & 0.08 \\
\hline hours & {$[0.03,0.36]$} & {$[0.07,0.52]$} & {$[0.00,0.36]$} & {$[0.04,0.23]$} & {$[0.01,0.14]$} & {$[0.01,0.09]$} & {$[0.02,0.13]$} \\
\hline Inflation & 0.05 & 0.37 & 0.43 & 0.05 & 0.08 & 0.02 & 0.01 \\
\hline & {$[0.00,0.12]$} & {$[0.16,0.54]$} & {$[0.22,0.65]$} & {$[0.01,0.09]$} & {$[0.02,0.13]$} & {$[0.00,0.03]$} & {$[0.00,0.02]$} \\
\hline Interest & 0.25 & 0.29 & 0.12 & 0.06 & 0.18 & 0.06 & 0.04 \\
\hline rate & {$[0.04,0.53]$} & {$[0.12,0.45]$} & {$[0.01,0.21]$} & {$[0.02,0.10]$} & {$[0.06,0.29]$} & {$[0.00,0.12]$} & {$[0.00,0.06]$} \\
\hline
\end{tabular}

\subsection{Conclusion}

At the heart of this chapter is an identification problem that affects the interpretation of the key drivers of the business cycle. We demonstrate that the introduction of financial frictions materially alters which shocks are thought to be the most important drivers of the business cycle. When entrepreneurs are subject to binding collateral constraints, a reduction in the value of installed capital reduces the value of collateral and thus the amount an entrepreneur can borrow. We find that the dynamic responses of output and consumption to a positive investment shock are materially altered by such collateral constraints. While an investment shock prompts more investment and positive output growth, the behaviour of consumption is completely altered, since the impact effect is for consumption to fall. The investment shock causes collateral values to decline, which reduces entrepreneurs' ability to obtain external finance. Thus, to increase investment entrepreneurs are forced to reduce their consumption. Investment shocks can then no longer generate the positive co-movement that is evident between consumption and invest- 
ment. Instead, in the model with collateral constraints, risk premium shocks increase markedly in importance, whereas shocks to investment have a much diminished role, contributing only 4 percent of the variation in output. 
Appendix 


\section{A Linearized model}

Table 4.A.1: Linearized model equations

\begin{tabular}{|c|c|}
\hline $\begin{array}{l}\text { GDP } \\
\text { Marginal utility c }\end{array}$ & $\begin{array}{l}y_{t}=\frac{c^{a}}{y} c_{t}^{a}+\frac{i}{y} i_{t}+\frac{z}{y} z_{t}+\varepsilon_{g, t} \\
\lambda_{t}=-\sigma^{c} \frac{1}{1-\underline{h}}\left(c_{t}-\frac{h}{\gamma} c_{t-1}\right)+\frac{\sigma^{c}-1}{1-\underline{h}}(W L C) l_{t}\end{array}$ \\
\hline Euler & $\lambda_{t}=\mathrm{E}_{t}\left(\lambda_{t+1}^{\gamma}+r_{t}+\varepsilon_{c, t}-\pi_{t+1}\right)^{\gamma}$ \\
\hline Marginal utility $c^{e}$ & $\lambda_{t}^{e}=-\sigma^{e} \frac{1}{1-\frac{h^{e}}{\gamma}}\left(c_{t}^{e}-\frac{h}{\gamma} c_{t-1}^{e}\right)$ \\
\hline Entrepreneur's Euler & $\lambda_{t}^{e}=\lambda_{t+1}^{e}+v\left(r_{t}+\varepsilon_{c, t}-\pi_{t+1}\right)+(v-1) \Delta_{t}$ \\
\hline Borrowing constraint & $b_{t}+r_{t}+\varepsilon_{c, t}-\pi_{t+1}=q_{t+1}+k_{t}$ \\
\hline Entrep. flow constr. & $\begin{array}{l}\frac{c^{e}}{y} c_{t}^{e}+\frac{i}{y} i_{t}=\frac{b}{y} b_{t}+\frac{b}{y} \frac{R R}{\gamma \pi}\left(\pi_{t}-b_{t-1}-r_{t-1}-\varepsilon_{c, t-1}\right) \\
\quad+\left(r k_{t}+k_{t-1}\right) r k \frac{k}{2} \frac{1}{\gamma}\end{array}$ \\
\hline Consumption agg. & $\frac{c^{a}}{y} c_{t}^{a}=\frac{c}{y} c_{t}+\frac{c^{e}}{y} c_{t}^{e}$ \\
\hline Investment & $\begin{aligned} i_{t}= & \frac{1}{1+\beta^{e} \gamma^{1-\sigma^{e}}} i_{t-1}+\left(1-\frac{1}{1+\beta^{e} \gamma^{1-\sigma^{e}}}\right) i_{t+1} \\
& +\frac{1}{1+\beta^{e} \gamma^{1-\sigma^{e}} \gamma^{2} \phi} q_{t}+\varepsilon_{\mu, t}\end{aligned}$ \\
\hline Tobin's q & $\begin{aligned} q_{t}= & \left((1-\delta) \beta^{e} \gamma^{-\sigma^{e}}+\Delta \chi\right) q_{t+1} \\
& +\left(1-(1-\delta) \beta^{e} \gamma^{-\sigma^{e}}-\Delta \chi\right) r k_{t+1} \\
& +\Delta \chi \Delta_{t}+\lambda_{t+1}^{e}-\lambda_{t}^{e}\end{aligned}$ \\
\hline Production fn. & $y_{t}=\phi_{F}\left(\alpha \bar{k}_{t}+(1-\alpha) l_{t}+\varepsilon_{a, t}\right)$ \\
\hline Effective capital & $\bar{k}_{t}=k_{t-1}+z_{t}$ \\
\hline Capital utilization & $z_{t}=\frac{1-\xi}{\xi} r k_{t}$ \\
\hline Capital accumulation & $\begin{aligned} k_{t}= & \frac{1-\delta}{\gamma} k_{t-1}+\left(1-\frac{1-\delta}{\gamma}\right) i_{t} \\
& +\left(1-\frac{1-\delta}{\gamma}\right)\left(1+\beta^{e} \gamma^{1-\sigma^{e}} \gamma^{2} \phi\right) \varepsilon_{\mu, t}\end{aligned}$ \\
\hline Marginal cost & $m c_{t}=(\alpha) r k_{t}+(1-\alpha) w_{t}-\varepsilon_{a, t}$ \\
\hline Wage mark up & $\mu_{t}^{w}=w_{t}-\left(\sigma^{l} l_{t}+\frac{1}{1-\frac{h}{\gamma}}\left(c_{t}-\frac{h}{\gamma} c_{t-1}\right)\right.$ \\
\hline Cost minimization & $r k_{t}=-\left(\bar{k}_{t}-l_{t}\right)+w_{t}$ \\
\hline Price inflation & $\begin{aligned} \pi_{t}= & \frac{\delta_{p}}{1+\beta \gamma^{1-\sigma^{c} \delta_{p}}} \pi_{t-1}+\frac{\beta \gamma^{1-\sigma^{c}}}{1+\beta \gamma^{1-\sigma^{c} \delta_{p}}} \pi_{t+1} \\
& +\left(\frac{1-\beta \gamma^{1-\sigma^{c}} \theta_{p}}{1+\beta \gamma^{1-\sigma^{c}} \delta_{p}}\right) \frac{1-\theta_{p}}{\left(\theta_{p}\left(\left(\phi_{p}-1\right) \epsilon_{p}+1\right)\right)} m c_{t}+\varepsilon_{p, t}\end{aligned}$ \\
\hline Wage inflation & $\begin{aligned} w_{t}= & \frac{1}{1+\beta \gamma^{1-\sigma^{c}}} w_{t-1}+\left(1-\frac{1}{1+\beta \gamma^{1-\sigma^{c}}}\right)\left(w_{t+1}+\pi_{t+1}\right) \\
& -\frac{1+\beta \gamma^{1-\sigma^{c}} \delta_{w}}{1+\beta \gamma^{1-\sigma^{c}}} \pi_{t}+\frac{\delta_{w}}{1+\beta \gamma^{1-\sigma^{c}}} \pi_{t-1} \\
& -\frac{1-\beta \gamma^{1-\sigma^{c}} \theta_{w}}{1+\beta \gamma^{1-\sigma^{c}}} \frac{1-\theta_{w}}{\theta_{w}\left(\left(\phi_{w}-1\right) \epsilon_{w}+1\right.} \mu_{t}^{w}+\varepsilon_{w, t}\end{aligned}$ \\
\hline Interest rate rule & $\begin{aligned} r_{t}= & \phi_{r} r_{t-1}+\left(1-\phi_{r}\right)\left(\phi_{\pi} \pi_{t}+\phi_{x}\left(y_{t}-y_{t}^{f l e x}\right)\right) \\
& +\phi_{d x}\left(y_{t}-y_{t-1}-\left(y_{t}^{\text {flex }}-y_{t-1}^{\text {flex }}\right)\right)+\varepsilon_{r, t}\end{aligned}$ \\
\hline
\end{tabular}

Note that $\Delta$ is the steady state value of the shadow price on the borrowing constraint; $v=\beta \gamma^{-\sigma_{c}} /\left(\beta_{E} \gamma^{-\sigma_{c E}}\right)$; and $\Delta_{t}$ is the multiplier on the borrowing constraint. 


\section{B.2 Data transformations}

We transform the data as described in Justiniano et al.'s Investment shocks and business cycles: technical appendix and additional results, with a minor exception relating to nonfarm labour hours (discussed below). The mnemonics from table 4.B.1 are used in the right hand side of table 4.B.2.

For per capita labour hours we use the LXNFH series instead of the HNFBN series reported by Justiniano et al. because the latter series no longer seems to be available in Haver. LXNFH is an index with a base year in 2005. We normalize our series to replicate the properties of the series in Justiniano et al.. ${ }^{10}$ In their sample $\ln (H N F B N /(L F+L H))$ appears to have has been normalized to zero. The Federal Funds rate is divided by 4 because the model represents quarterly data. No other demeaning or de-trending is performed on the data.

\footnotetext{
${ }^{10}$ The choice of parameters is very slightly modified to those found from regressing the data from Justiniano et al. on $\ln (L X N F H /(L F+L H))$.
} 


\section{B Data}

\section{B.1 Data sources}

Table 4.B.1: Raw data

\begin{tabular}{ll} 
Mnemonic & Description \\
\hline GDP & Gross Domestic Product (SAAR, Bil.\$) \\
JGDP & Gross Domestic Product: Chain Price Index (SA, 2005=100) \\
CN & Personal Consump. Expend.: Nondurable Goods (SAAR, Bil.\$) \\
CS & Personal Consump. Expend.: Services (SAAR, Bil.\$) \\
CD & Personal Consump. Expend.: Durable Goods (SAAR, Bil.\$) \\
I & Gross Private Domestic Investment (SAAR, Bil. $\$)$ \\
LF & Civilian Labor Force: 16 yr $+($ SA, Thous) \\
LH & Not in the Labor Force: 16 yr $+($ SA, Thous) \\
LXNFC & Nonfarm Business Sector: Compensation/Hour $(\mathrm{SA}, 2005=100)$ \\
LXNFH & Nonfarm Business Sector: Hours of All Persons $(\mathrm{SA}, 2005=100)$ \\
FFED & Federal Funds [effective] Rate $(\%$ p.a.) \\
\hline
\end{tabular}

Note: All data are sourced from Haver. The Haver mnemonics in the first column should be suffixed with @USECON to call the series from the Haver Excel add-in.

Table 4.B.2: Data transformations

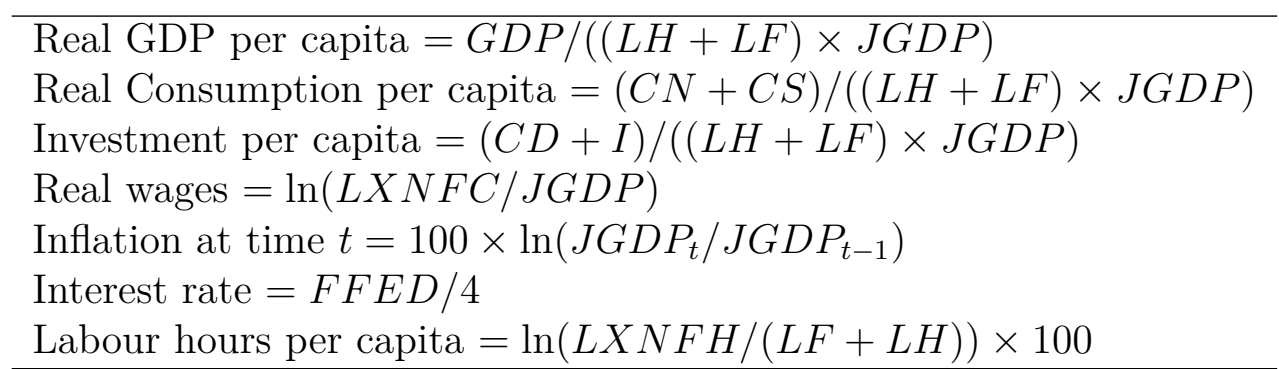

Notes: The observables for Real GDP per capita, real consumption per capita, real investment per capita, and real wages are computed as 100 times the log difference of each of the series described above, ie the log approximation of quarterly percent changes. 


\section{C MCMC convergence}

This appendix illustrates diagnostics to assess whether the Markov chains have converged to the posterior distributions of interest. Two Markov chains were iterated 900,000 times to estimate the posterior distribution of the vector of parameter. As noted by Brooks and Roberts (1998) and others, no diagnostic can guarantee convergence. Nevertheless, these figures provide some reassurance that the chains have indeed converged to their stationary distributions. The figures below illustrate the deciles for each chain, computed recursively as the chains are iterated forward (following a 100,000 burn-in period to reduce bias from initial conditions). Convergence implies that like-deciles from the two chain should asymptote to the same values, which should remain constant as the sample is extended. The variability evident in some deciles for some parameters seems fairly modest and is unlikely to be of economic significance. 
Figure 4.C.1: MCMC convergence - deciles (Panel A)
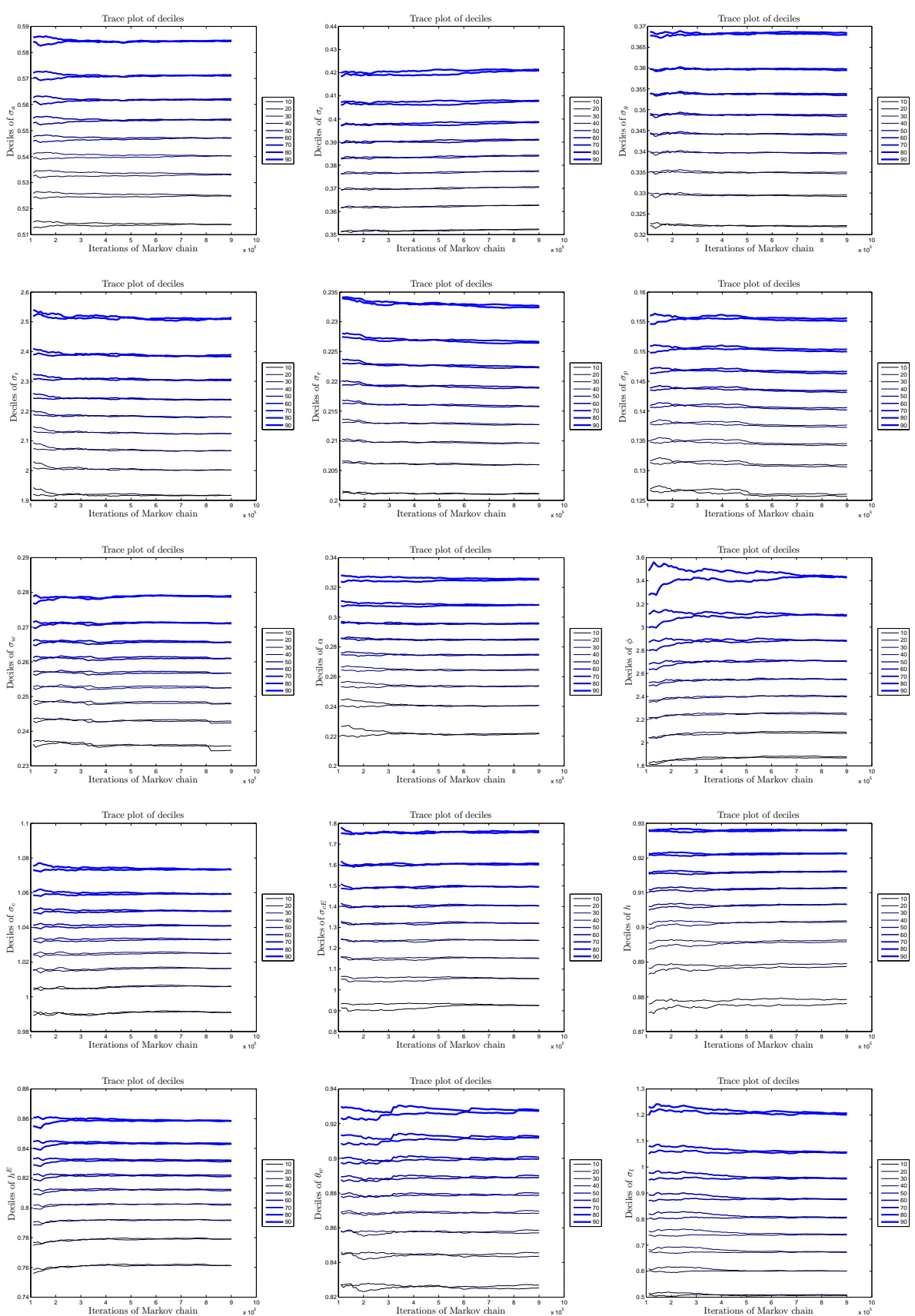
Figure 4.C.2: MCMC convergence - deciles (Panel B)
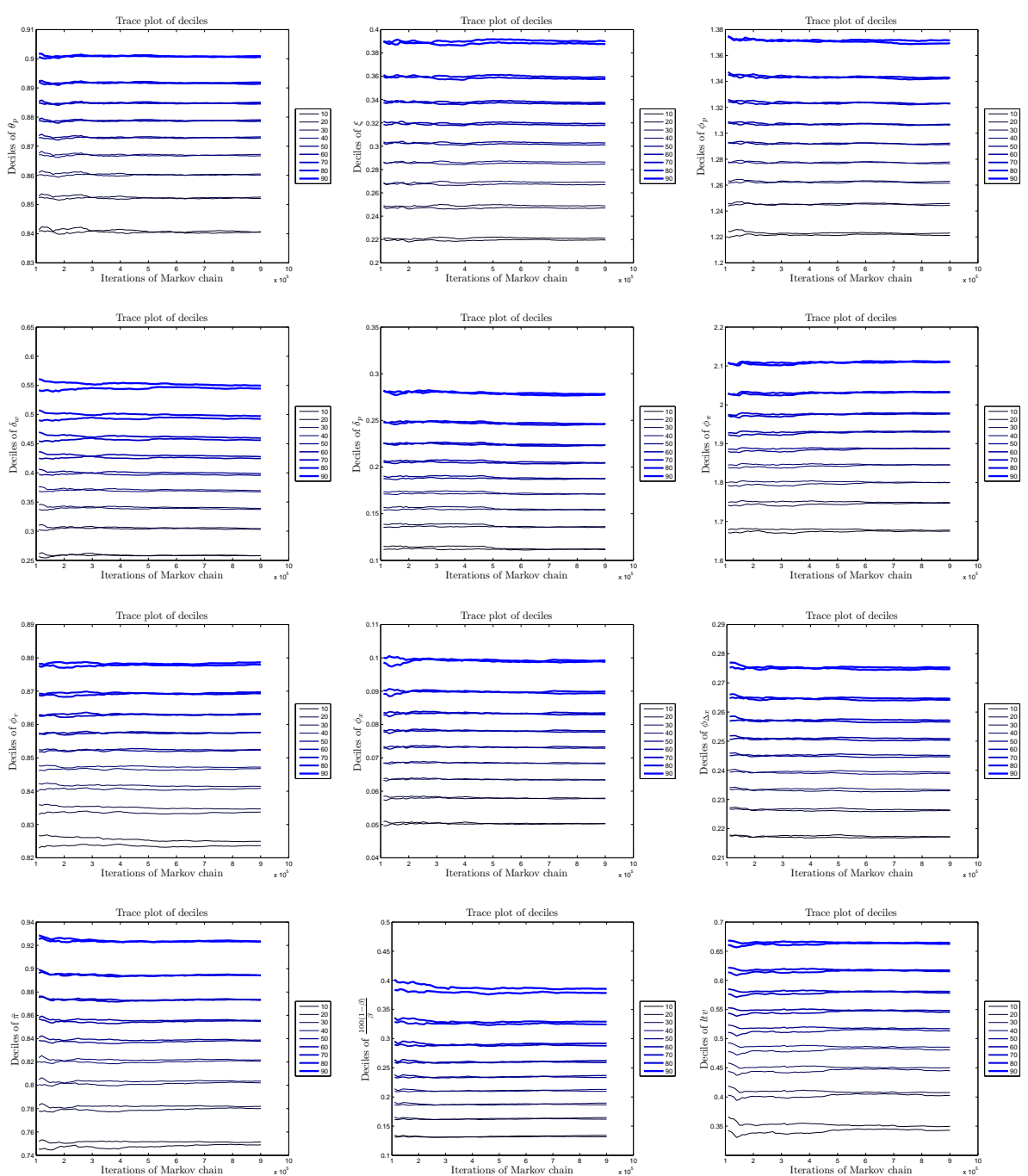

Trace plot of deciles
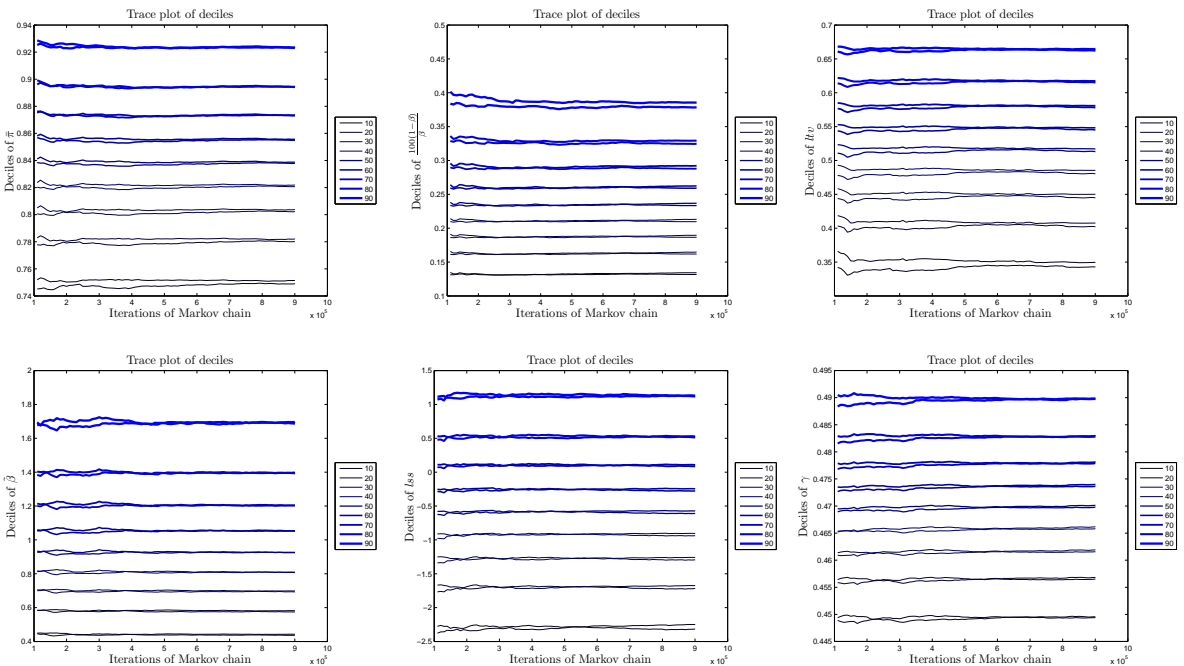
Figure 4.C.3: MCMC convergence - deciles (Panel C)
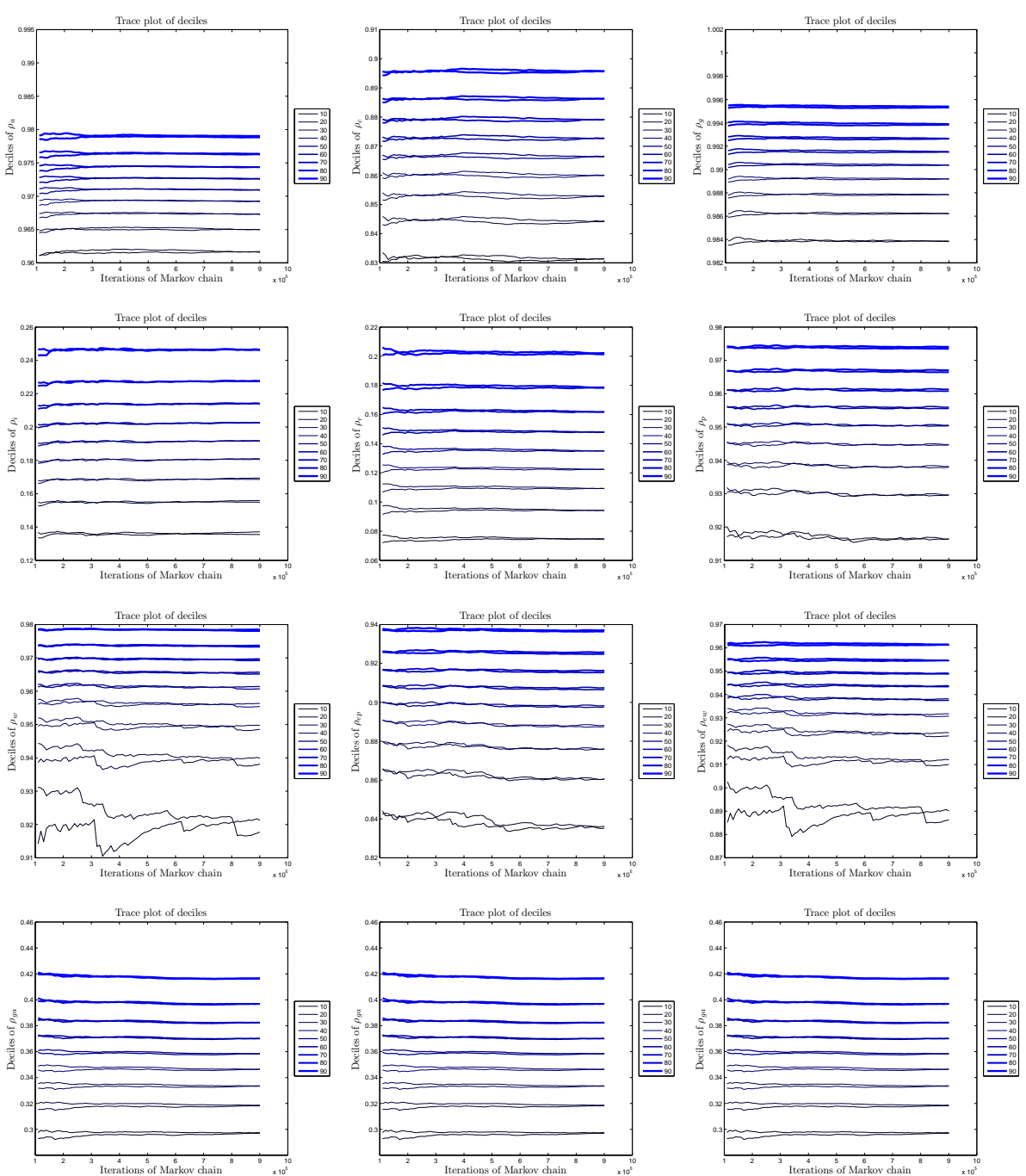

Trace plot of deciles

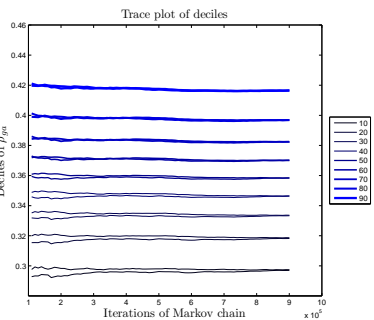




\section{Bibliography}

Adjemian, S., Bastani, H., Karamé, F., Juillard, M., Maih, J., Mihoubi, F., Perendia, G., Pfeifer, J., Ratto, M., and Villemot, S. (2011). Dynare: Reference Manual Version 4. Dynare Working Papers 1, CEPREMAP.

Adolfson, M. (2007). Incomplete exchange rate pass-through and simple monetary policy rules. Journal of International Money and Finance, 26(3):468 $-494$.

Aiyagari, S. R. and Gertler, M. (1999). Overreaction of asset prices in general equilibrium. Review of Economic Dynamics, 2(1):3-35.

Aldrich, J. (1989). Autonomy. Oxford Economic Papers, 41(1):15-34.

Aldrich, J. (2002). How likelihood and identification went Bayesian. International Statistical Review / Revue Internationale de Statistique, 70(1):79-98.

Altig, D., Christiano, L., Eichenbaum, M., and Linde, J. (2011). Firm-specific capital, nominal rigidities and the business cycle. Review of Economic Dynamics, 14(2):225-247.

Altman, M., editor (2006). Handbook of Contemporary Behavioral Economics: Foundations and Developments. M.E. Sharpe Inc., Armonk, New York.

Altug, S. (1989). Time-to-build and aggregate fluctuations: Some new evidence. International Economic Review, 30:889-920.

An, S. and Schorfheide, F. (2007). Bayesian analysis of DSGE models. Econometric Reviews, 26(2-4):113-72. 
Andreasen, M. M., Fernández-Villaverde, J., and Rubio-Ramírez, J. (2018). The pruned state-space system for non-linear DSGE models: Theory and empirical applications. Review of Economic Studies, 85(1):1-49.

Araújo, E. (2012). Investment-specific shocks and real business cycles in emerging economies: Evidence from Brazil. Economic Modelling, 29(3):671-678.

Arrow, K. J. (1974). Limited knowledge and economic analysis. American Economic Review, 64(1):1-10.

Barrell, R., Fitzgerald, J., and Riley, R. (2010). EU enlargement and migration: Assessing the macroeconomic impacts. Journal of Common Market Studies, 48(2):373-395.

Barro, R. J. and King, R. G. (1984). Time-separable preferences and intertemporal substitution models of business cycles. Quarterly Journal of Economics, 99:817-839.

Bauwens, L., Lubrano, M., and Richard, J.-F. (1999). Bayesian Inference in Dynamic Econometric Models. Oxford University Press, Oxford.

Bayoumi, T. (2004). GEM: A new international macroeconomic model. Occasional Papers 239, International Monetary Fund. With assistance from D. Laxton, H. Faruqee, B. Hunt, P. Karam, J. Lee, A. Rebucci, and I. Tchakarov.

Beně̌, J., Binning, A., Fukač, M., Lees, K., and Matheson, T. (2009). K.I.T.T.: Kiwi Inflation Targeting Technology. Reserve Bank of New Zealand, Wellington, NZ.

Benigno, G. and Thoenissen, C. (2003). Equilibrium exchange rates and supply-side performance. Economic Journal, 113(486):C103-C124.

Berger, A. N. and Udell, G. F. (1990). Collateral, loan quality and bank risk. Journal of Monetary Economics, 25:21-42.

Berger, J. O. and Wolpert, R. L. (1988). The Likelihood Principle. Institute of Mathematical Statistics, Hayward, CA, 2nd edition. 
Bernanke, B. S., Gertler, M., and Gilchrist, S. (1996). The financial accelerator and the flight to quality. The Review of Economics and Statistics, 78(1):1-15.

Bernanke, B. S., Gertler, M., and Gilchrist, S. (1999). The financial accelerator in a quantitative business cycle framework. In Taylor, J. B. and Woodford, M., editors, Handbook of Macroeconomics, volume 1 of Handbook of Macroeconomics, chapter 21, pages 1341-1393. Elsevier.

Bierens, H. J. (2007). Econometric analysis of linearized singular dynamic stochastic general equilibrium models. Journal of Econometrics, $136(2): 595-627$.

Binder, M. and Pesaran, M. H. (1997). Multivariate linear rational expectations models: Characterization of the nature of the solutions and their fully recursive computation. Econometric Theory, 13(6):877-888.

Binder, M. J. and Pesaran, M. H. (1995). Multivariate rational expectations models and macroeconometric modeling: A review and some new results. In Pesaran, M. H. and Wickens, M., editors, Handbook of Applied Econometrics: Macroeconomics, pages 139-187. Blackwell Publishers, Oxford.

Bonaldi, J. P. (2010). Identification problems in the solution of linearized DSGE models. Borradores de Economia 593, Banco de la Republica de Colombia.

Borjas, G. J. (1994). The economics of immigration. Journal of Economic Literature, 32(4):1667-1717.

Borjas, G. J. (1999a). The economic analysis of immigration. In Ashenfelter, O. C. and Card, D., editors, Handbook of Labour Economics, volume 3A, chapter 28, pages 1697-1760. Elsevier, Amsterdam.

Borjas, G. J. (1999b). Heaven's Door: Immigration Policy and the American Economy. Princeton University Press, Princeton, NJ.

Borjas, G. J. (2014). Immigration Economics. Harvard University Press, Cambridge, MA. 
Borjas, G. J. (2017). The wage impact of the Marielitos: A reappraisal. International Labor Relations Review, 70(5):1077-1110.

Boubtane, E., Dumont, J.-C., and Rault, C. (2016). Immigration and economic growth in the OECD countries: 1986 - 2006. Oxford Economic Papers, 68(2):340-360.

Bowden, R. (1973). The theory of parametric identification. Econometrica, 41(6):1069-74.

Brooks, S. P. and Roberts, G. O. (1998). Convergence assessment techniques for Markov chain Monte Carlo. Statistics and Computing, 8(4):319-335.

Brubakk, L., Husebø, T. A., Maih, J., Olsen, K., and Østnor, M. (2006). Finding NEMO: Documentation of the Norwegian economy model. Staff Memo 2006/6, Norges Bank.

Brunow, S., Nijkamp, P., and Poot, J. (2015). The impact of international migration on economic growth in the global economy. In Chiswick, B. R. and Miller, P. W., editors, Handbook of the Economics of International Migration, volume 1B of Handbook of the Economics of International Migration, pages 1027 - 1075. North-Holland.

Brzoza-Brzezina, M. and Kolasa, M. (2013). Bayesian evaluation of DSGE models with financial frictions. Journal of Money, Credit and Banking, 45(8):1451-1476.

Brzoza-Brzezina, M., Kolasa, M., and Makarski, K. (2013). The anatomy of standard DSGE models with financial frictions. Journal of Economic Dynamics and Control, 37(1):32-51.

Burstein, A., Hanson, G., Tian, L., and Vogel, J. (2017). Tradability and the labor-market impact of immigration: Theory and evidence from the U.S. Working Paper 23330, National Bureau of Economic Research.

Calvo, G. A. (1983). Staggered prices in a utility-maximizing framework. Journal of Monetary Economics, 12(3):383-398. 
Canova, F. (2007). Methods for Applied Macroeconomic Research. Princeton University Press, Princeton.

Canova, F., Ferroni, F., and Matthes, C. (2014). Choosing the variables to estimate singular DSGE models. Journal of Applied Econometrics, 29(7):1099-1117.

Canova, F. and Sala, L. (2009). Back to square one: Identification issues in DSGE models. Journal of Monetary Economics, 56(4):431-449.

Card, D. (1990). The impact of the Mariel boatlift on the Miami labor market. Industrial and Labor Relations Review, 43(2):245-257.

Carlstrom, C. T. and Fuerst, T. S. (1997). Agency costs, net worth, and business fluctuations: A computable general equilibrium analysis. American Economic Review, 87(5):893-910.

Carroll, C. D. and Samwick, A. A. (1997). The nature of precautionary wealth. Journal of Monetary Economics, 40(1):41-72.

Cartwright, E. (2011). Behavioral Economics. Routledge, London.

Chari, V. V., Kehoe, P. J., and McGrattan, E. R. (2009). New Keynesian models: Not yet useful for policy analysis. American Economic Journal: Macroeconomics, 1(1):242-66.

Chen, B. (2016). Identification and overidentification of linear structural equation models. In Lee, D. D., Sugiyama, M., von Luxburg, U., Guyon, I., and Garnett, R., editors, Advances in Neural Information Processing Systems, volume 29, pages 1579-1587. Curran Associates, Inc.

Chiswick, B. R. and Miller, P. W., editors (2015). Handbook of the Economics of International Migration, volume 1A-1B. North-Holland, Oxford.

Christ, C. F. (1966). Econometric Models and Methods. John Wiley \& Sons, N.Y.

Christensen, I. and Dib, A. (2008). The financial accelerator in an estimated New Keynesian model. Review of Economic Dynamics, 11(1):155-178. 
Christiano, L. J. (2012). Christopher A. Sims and vector autoregressions. Scandinavian Journal of Economics, 114(4):1082-1104.

Christiano, L. J., Eichenbaum, M., and Evans, C. L. (2005). Nominal rigidities and the dynamic effects of a shock to monetary policy. Journal of Political Economy, 113(1):1-45.

Christiano, L. J., Eichenbaum, M., and Trabandt, M. (2018). On DSGE models. Journal of Economic Perspectives, 32(3):113-140.

Christiano, L. J., Motto, R., and Rostagno, M. (2012). Risk shocks. Mimeo, Northwestern University.

Christiano, L. J., Motto, R., and Rostagno, M. (2014). Risk shocks. American Economic Review, 104(1):27-65.

Christiano, L. J., Trabandt, M., and Walentin, K. (2011). Introducing financial frictions and unemployment into a small open economy model. Journal of Economic Dynamics and Control, 35(12):1999-2041.

Clark, X., Hatton, T. J., and Williamson, J. G. (2007). Explaining US immigration, 1971-1998. Review of Economics and Statistics, 89(2):359373.

Coleman, A. and Landon-Lane, J. (2007). Housing markets and migration in New Zealand, 1962-2006. Reserve Bank of New Zealand Discussion Paper Series DP2007/12, Reserve Bank of New Zealand.

Constant, A. F. and Zimmermann, K. F., editors (2013). International Handbook on the Economics of International Migration. Edward Elgar, Cheltenham.

Cúrdia, V. and Reis, R. (2010). Correlated disturbances and U.S. business cycles. Working Paper 15774, National Bureau of Economic Research.

De Paoli, B. (2009). Monetary policy and welfare in a small open economy. Journal of International Economics, 77(1):11-22.

DeJong, D. N. and Dave, C. (2007). Structural Macroeconometrics. Princeton University Press, Princeton. 
Del Negro, M. and Schorfheide, F. (2004). Priors from general equilibrium models for VARs. International Economic Review, 45(2):643-673.

Del Negro, M. and Schorfheide, F. (2008). Forming priors for DSGE models (and how it affects assessment of nominal rigidities). Journal of Monetary Economics, 55(7):1191-1208.

Diebold, F. X. (1998). The past, present and future of macroeconomic forecasting. Journal of Economic Perspectives, 12:175-92.

DOL (2009). New Faces, New Futures, New Zealand: Findings from the Longitudinal Immigration Survey. Department of Labour, Wellington, NZ.

Drèze, J. H. (1972). Econometrics and decision theory. Econometrica, 40(1):1-18.

Drèze, J. H. (1975a). Bayesian theory of identification in simultaneous equations models. In Fienberg, S. A. and Zellner, A., editors, Studies in Bayesian Econometrics and Statistics, pages 159-174. North Holland Publishing Company, Amsterdam.

Drèze, J. H. (1975b). Econometrics and decision theory. In Fienberg, S. A. and Zellner, A., editors, Studies in Bayesian Econometrics and Statistics, pages 17-38. North Holland Publishing Company, Amsterdam.

Dufour, J.-M., Khalaf, L., and Kichian, M. (2013). Identification-robust analysis of DSGE and structural macroeconomic models. Journal of Monetary Economics, 60(3):340-350.

Dustmann, C., Fabbri, F., and Preston, I. (2005). The impact of immigration on the British labour market. The Economic Journal, 115(507):F324-F341.

Engle, R. F. and Granger, C. (1987). Co-integration and error correction: Representation, estimation, and testing. Econometrica, 55(2):251-276.

Erceg, C. J., Henderson, D. W., and Levin, A. T. (2000). Optimal monetary policy with staggered wage and price contracts. Journal of Monetary Economics, 46:281-313. 
Eusepi, S. and Preston, B. (2011). Expectations, learning, and business cycle fluctuations. American Economic Review, 101(6):2844-2872.

Evans, G. W. and Honkapohja, S. (2010). Learning and Expectations in Macroeconomics. Princeton University Press, Princeton, NJ.

Farmer, R. E. A., Khramov, V., and Nicolò, G. (2015). Solving and estimating indeterminate DSGE models. Journal of Economic Dynamics and Control, 54:17-36.

Fernández-Villaverde, J. (2010). The econometrics of DSGE models. SERIEs, $1(1): 3-49$.

Fernández-Villaverde, J., Guerrón-Quintana, P., and Rubio-Ramírez, J. F. (2010). The new macroeconometrics: A Bayesian approach. In O'Hagan, A. and West, M., editors, The Oxford Handbook of Applied Bayesian Analysis, chapter 15. Oxford University Press, Oxford.

Fernández-Villaverde, J., Rubio-Ramírez, J. F., and Schorfheide, F. (2016). Solution and estimation methods for DSGE models. In Taylor, J. B. and Uhlig, H., editors, Handbook of Macroeconomics, volume 2A, chapter 9, pages 527-724. Elsevier, North Holland.

Fischer, S. (1977). Long-term contracts, rational expectations, and the optimal money supply rule. Journal of Political Economy, 85:191-205.

Fisher, J. D. M. (2006). The dynamic effects of neutral and investmentspecific technology shocks. Journal of Political Economy, 114(3):413-451.

Florens, J.-P., Marimoutou, V., and Péguin-Feissolle, A. (2007). Econometric Modeling and Inference. Cambridge University Press, Cambridge.

Friedman, M. (1957). A Theory of the Consumption Function. National Bureau of Economic Research. Princeton University Press, Princeton, NJ.

Friedman, M. (1968). The role of monetary policy. American Economic Review, 58(1):1-17. 
Fromm, G., Klein, L. R., and Schink, G. R. (1972). Short- and long-term simulations with the Brookings model. In Hickman, B. G., editor, Econometric Models of Cyclical Behaviour, Vols. 1 and 2, pages 201-310. Columbia University Press on behalf of National Bureau of Economic Research, New York.

Fuentes-Albero, C. (2018). Financial frictions, financial shocks, and aggregate volatility. Journal of Money, Credit and Banking. forthcoming.

Fuhrer, J. C. (2000). Habit formation in consumption and its implications for monetary-policy models. American Economic Review, 90(3):367-390.

Fukač, M. and Pagan, A. (2007). Commentary on "An estimated DSGE model for the United Kingdom". Review, Federal Reserve Bank of St. Louis.

Furlanetto, F. and Robstad, Ø. (2016). Immigration and the macroeconomy: Some new empirical evidence. Working Paper 18, Norges Bank.

Furlanetto, F. and Seneca, M. (2014). Investment shocks and consumption. European Economic Review, 66:111-127.

Gabrielsen, A. (1978). Consistency and identifiability. Journal of Econometrics, 8(2):261-263.

Galí, J., López-Salido, J. D., and Vallés, J. (2007). Understanding the effects of government spending on consumption. Journal of the European Economic Association, 5(1):227-70.

Galí, J. and Monacelli, T. (2005). Monetary policy and exchange rate volatility in a small open economy. Review of Economic Studies, 72(3):707-734.

Gelman, A., Carlin, J. B., Stern, H. S., and Rubin, D. B. (2004). Bayesian Data Analysis. Chapman \& Hall/CRC, Boca Raton, FL, 2nd edition.

Gerali, A., Neri, S., Sessa, L., and Signoretti, F. M. (2010). Credit and banking in a DSGE model of the euro area. Journal of Money, Credit and Banking, 42(s1):107-141. 
Gertler, M. and Karadi, P. (2011). A model of unconventional monetary policy. Journal of Monetary Economics, 58(1):17-34.

Geweke, J. (2005). Contemporary Bayesian Econometrics and Statistics. John Wiley and Sons Ltd, Hoboken, New Jersey.

Gibson, J., Rohorua, H., McKenzie, D., and Stillman, S. (2018). The longterm impacts of international migration: Evidence from a lottery. World Bank Economic Review, 32(1):127-147.

Goldfeld, S. M. and Sichel, D. E. (1990). The demand for money. In Friedman, B. M. and Hahn, F. H., editors, Handbook of Monetary Economics, volume 1, chapter 8, pages 288-356. North-Holland, New York.

Golub, G. H. and Loan, C. F. V. (1996). Matrix Computations. The Johns Hopkins University Press, Baltimore, MD, 3rd edition.

Granger, C. and Newbold, P. (1974). Spurious regressions in econometrics. Journal of Econometrics, 2(2):111-120.

Grauwe, P. D. (2010). The scientific foundation of dynamic stochastic general equilibrium (dsge) models. Public Choice, 144(3/4):413-443.

Greene, W. H. (1997). Econometric Analysis. Prentice Hall, Upper Saddle River, NJ.

Greenwood, J., Hercowitz, Z., and Krusell, P. (2000). The role of investmentspecific technological change in the business cycle. European Economic Review, 44(1):91-115.

Gu, C., Mattesini, F., Monnet, C., and Wright, R. (2013). Banking: A new monetarist approach. Review of Economic Studies, 80(2):636-662.

Guerron-Quintana, P. A. (2010). What you match does matter: The effects of data on DSGE estimation. Journal of Applied Econometrics, 25(5):774804.

Hall, R. E. (2011). The high sensitivity of economic activity to financial frictions. The Economic Journal, 121:351-378. 
Hall, R. E. (2013). Financial frictions. International Journal of Central Banking, 9(2):155-163.

Hamilton, J. D. (2017). Why you should never use the Hodrick-Prescott filter. Review of Economics and Statistics. Forthcoming.

Hannan, E. J. and Deistler, M. (1988). The Statistical Theory of Linear Systems. John Wiley \& Sons, New York.

Harhoff, D. and Korting, T. (1998). Lending relationship in Germany Empirical evidence from survey data. Journal of Banking and Finance, 22(10-11):1317-1353.

Hartley, J. E., Hoover, K. D., and Salyer, K. D., editors (1998). Real Business Cycles: A Reader. Routledge, London.

Hatanaka, M. (1975). On the global identification of the dynamic simultaneous equations model with stationary disturbances. International Economic Review, 16(3):545-554.

Hatton, T. J. (1995). A model of U.K. emigration, 1870-1913. The Review of Economics and Statistics, 77(3):407-415.

Heer, B. and Maußner, A. (2009). Dynamic General Equilibrium Modeling: Computational Methods and Applications. Springer-Verlag, Berlin, 2nd edition.

Herbst, E. P. and Schorfheide, F. (2014). Sequential Monte Carlo sampling for DSGE models. Journal of Applied Econometrics, 29(7):1073-1098.

Herbst, E. P. and Schorfheide, F. (2015). Bayesian Estimation of DSGE Models. Princeton University Press, Princeton, NJ.

Hicks, J. R. (1937). Mr Keynes and the "classics"; A suggested interpretation. Econometrica, 5(2):147-159.

Hirakata, N., Sudo, N., and Ueda, K. (2011). Do banking shocks matter for the U.S. economy? Journal of Economic Dynamics and Control, 35(12):2042-2063. 
Hodgson, R. and Poot, J. (2011). New Zealand Research on the Economic Impacts of Immigration 2005-2010: Synthesis and Research Agenda. CReAM Discussion Paper Series 1104, Centre for Research and Analysis of Migration (CReAM), Department of Economics, University College London.

Hollmayr, J. and Kuhl, M. (2016). Imperfect information about financial frictions and consequences for the business cycle. Review of Economic Dynamics, 22:179-207.

Hsiao, C. (1983). Identification. In Griliches, Z. and Intriligator, M. D., editors, Handbook of Econometrics, volume 1, pages 223-283. Elsevier, Amsterdam.

Iacoviello, M. (2005). House prices, borrowing constraints, and monetary policy in the business cycle. American Economic Review, 95(3):739-764.

Iacoviello, M. and Neri, S. (2010). Housing market spillovers: Evidence from an estimated DSGE model. American Economic Journal: Macroeconomics, 2(2):125-164.

Ireland, P. (2004a). A method for taking models to the data. Journal of Economic Dynamics and Control, 28(6):1205-1226.

Ireland, P. (2004b). Technology shocks in the New Keynesian model. Review of Economics and Statistics, 86(4):923-936.

Iskrev, N. (2010a). Evaluating the strength of identification in DSGE models. An a priori approach. Working Paper 32, Banco de Portugal.

Iskrev, N. (2010b). Local identification in DSGE models. Journal of Monetary Economics, 57(2):189-202.

Iskrev, N. and Ritto, J. (2016). Choosing the variables to estimate singular DSGE models: Comment. Mimeo, Banco de Portugal.

Jacob, P. and Munro, A. (2018). A prudential stable funding requirement and monetary policy in a small open economy. Journal of Banking and Finance, 94:89-106. 
Jermann, U. and Quadrini, V. (2012). Macroeconomic effects of financial shocks. American Economic Review, 102(1):238-271.

Jerome, H. (1926). Migration and Business Cycles. National Bureau of Economic Research, New York.

Jimenez, G., Salas, V., and Saurina, J. (2006). Determinants of collateral. Journal of Financial Economics, 81(2):255-281.

Johansen, S. (1988). Statistical analysis of cointegrated vectors. Journal of Economic Dynamics and Control, 12(1-2):231-254.

Judd, J. P. and Scadding, J. L. (1982). The search for a stable money demand function: A survey of the post-1973 literature. Journal of Economic Literature, 20(3):993-1023.

Judd, K. L. (1999). Numerical Methods in Economics. MIT Press, Cambridge, MA.

Justiniano, A. and Preston, B. (2006). Can structural small open economy models account for the influence of foreign disturbances? Working Paper 12/2006, Centre for Applied Macroeconomic Analysis.

Justiniano, A. and Preston, B. (2008). Can structural small open economy models account for the influence of foreign disturbances? NBER Working Papers 14547, National Bureau of Economic Research.

Justiniano, A. and Preston, B. (2010). Can structural small open-economy models account for the influence of foreign variables? Journal of International Economics, 81(1):61-74.

Justiniano, A., Primiceri, G., and Tambalotti, A. (2011). Investment shocks and the relative price of investment. Review of Economic Dynamics, 14(1):101-121.

Justiniano, A., Primiceri, G. E., and Tambalotti, A. (2010). Investment shocks and business cycles. Journal of Monetary Economics, 57(2):132145 . 
Kadane, J. B. (1975). The role of identification in Bayesian theory. In Fienberg, S. E. and Zellner, A., editors, Studies in Bayesian Econometrics and Statistics, pages 175-191. North-Holland, Amsterdam.

Kagel, J. H. and Roth, A. E. (2016a). The Handbook of Experimental Economics, volume 1. Princeton University Press, Princeton.

Kagel, J. H. and Roth, A. E. (2016b). The Handbook of Experimental Economics, volume 2. Princeton University Press, Princeton.

Kamber, G., Smith, C., and Thoenissen, C. (2015). Financial frictions and the role of investment-specific technology shocks in the business cycle. Economic Modelling, 51:571-582.

Kapetanios, G., Pagan, A., and Scott, A. (2007). Making a match: Combining theory and evidence in policy-oriented macroeconomic modeling. Journal of Econometrics, 136(2):565-594.

Karagedikli, O., Matheson, T. D., Smith, C., and Vahey, S. (2010). RBCs and DSGEs: The computational approach to business cycle theory and evidence. Journal of Economic Surveys, 24(1):113-136.

Kass, R. E. and Raftery, A. E. (1995). Bayes factors. Journal of the American Statistical Association, 90(430):773-795.

Kerr, S. P. and Kerr, W. R. (2011). Economic impacts of immigration: A survey. Finnish Economic Papers, 24(1):1-32.

Keynes, J. M. (1936). The General Theory of Employment, Interest and Money. The Macmillan Press Ltd, London and Basingstoke.

Keynes, J. M. (1937). The general theory of employment. Quarterly Journal of Economics, 51(2):209-223.

Khan, H. and Tsoukalas, J. (2011). Investment shocks and the comovement problem. Journal of Economic Dynamics and Control, 35(1):115-130.

Kim, D. and Lee, C.-I. (2007). On-the-job human capital accumulation in a real business cycle model: Implications for intertemporal substitution 
elasticity and labor hoarding. Review of Economic Dynamics, 10(3):494518.

Kim, J., Kim, S., Schaumburg, E., and Sims, C. A. (2008). Calculating and using second-order accurate solutions of discrete time dynamic equilibrium models. Journal of Economic Dynamics and Control, 32(11):3397-3414.

Kim, K. and Pagan, A. R. (1995). The econometric analysis of calibrated macroeconomic models. In Pesaran, M. H. and Wickens, M. R., editors, Handbook of Applied Econometrics: Macroeconomics, volume 1, pages 356390. Blackwell Publishers, Oxford.

Kimball, M. (1995). The quantitative analytics of the basic neomonetarist model. Journal of Money, Credit and Banking, 27(4):1241-1277.

Kirsanova, T., Leith, C., and Wren-Lewis, S. (2006). Should central banks target consumer prices or the exchange rate? The Economic Journal, 116:F208-F231.

Kiyotaki, N. and Moore, J. (1997). Credit cycles. Journal of Political Economy, 105(2):211-248.

Klein, P. (2000). Using the generalized Schur form to solve a multivariate linear rational expectations model. Journal of Economic Dynamics and Control, 24(10):1405-1423.

Kolasa, M. and Rubaszek, M. (2015). Forecasting using DSGE models with financial frictions. International Journal of Forecasting, 31(1):1-19.

Komunjer, I. and Ng, S. (2011). Dynamic identification of dynamic stochastic general equilibrium models. Econometrica, 79(6):1995-2032.

Koop, G. (2003). Bayesian Econometrics. John Wiley and Sons Ltd, Chichester, Sussex.

Koop, G., Pesaran, M. H., and Smith, R. P. (2013). On identification of Bayesian DSGE models. Journal of Business and Economic Statistics, 31(3):300-314. 
Koopmans, T. C. (1953). Identification problems in economic model construction. In Hood, W. C. and Koopmans, T. C., editors, Studies in Econometric Method, chapter II, pages 27-48. John Wiley and Sons, New York.

Koopmans, T. C. and Reiers $\varnothing l$, O. (1950). The identification of structural characteristics. Annals of Mathematical Statistics, 21(2):165-181.

Krogh, T. S. (2015). Macro frictions and theoretical identification of the New Keynesian Phillips curve. Journal of Macroeconomics, 43:191-204.

Kydland, F. E. and Prescott, E. C. (1982). Time to build and aggregate fluctuations. Econometrica, 50(6):1345-1370.

Lai, H. (2008). Maximum likelihood estimation of singular systems of equations. Economics Letters, 99(1):51-54.

Leeson, R. (1997). The trade-off interpretation of Phillips's dynamic stabilization exercise. Economica, 64(253):155-171.

Lester, R. A. (1946). Shortcomings of marginal analysis for wage-employment problems. American Economic Review, 36(1):63-82.

Lester, R. A. (1947). Marginalism, minimum wages, and labor markets. American Economic Review, 37(1):135-148.

Lombardo, G. and McAdam (2012). Financial market frictions in a model of the Euro area. Economic Modelling, 29(6):2460-2485.

Lubik, T. and Schorfheide, F. (2004a). Computing sunspot equilibria in linear rational expectations models. Journal of Economic Dynamics and Control, 28(2):273-285.

Lubik, T. A. and Schorfheide, F. (2004b). Testing for indeterminacy: An application to U.S. monetary policy. American Economic Review, 94(1):190219.

Lucas, Jr., R. E. (1976). Econometric policy evaluation: A critique. Carnegie Rochester Conference Series on Public Policy, 1:19-46. 
Lütkepohl, H. (1994). The sources of the U.S. money demand instability. In Dufour, J.-M. and Raj, B., editors, New Developments in Time Series in Econometrics, pages 173-187. Physica-Verlag, Heidelberg.

Lütkepohl, H. (2006). New Introduction to Multiple Time Series Analysis. Springer, Berlin.

Machlup, F. (1947a). Rejoinder to an antimarginalist. American Economic Review, 36(1).

Machlup, F. (1947b). Rejoinder to an antimarginalist. American Economic Review, 37(1):148-154.

Mandelman, F. S. and Zlate, A. (2012). Immigration, remittances and business cycles. Journal of Monetary Economics, 59(2):196-213.

Mankiw, N. G. (1990). A quick refresher course in macroeconomics. Journal of Economic Literature, 20:1645-1660.

Mankiw, N. G. and Reis, R. (2002). Sticky information versus sticky prices: A proposal to replace the New Keynesian Phillips curve. Quarterly Journal of Economics, 117(4):1295-1328.

Mankiw, N. G. and Reis, R. (2010). Imperfect information and aggregate supply, volume 3A. North Holland, Amsterdam.

Mankiw, N. G. and Romer, D., editors (1991a). New Keynesian Economics: Coordination Failures and Real Rigidities, volume 2. MIT Press, Boston, MA.

Mankiw, N. G. and Romer, D., editors (1991b). New Keynesian Economics: Imperfect Competition and Sticky Prices, volume 1. MIT Press, Boston, MA.

Manski, C. F. (1993). Identification problems in the social sciences. Sociological Methodology, 23:1-56.

Manski, C. F. (1995). Identification Problems in the Social Sciences. Harvard University Press, Cambridge, MA. 
Manski, C. F. (2003). Identification problems in the social sciences and everyday life. Southern Economic Journal, 70(1):11-21.

Manski, C. F. (2007). Identification for Prediction and Decision. Harvard University Press, Cambridge, MA.

Mayda, A. M. (2010). International migration: A panel data analysis of the determinants of bilateral flows. Journal of Population Economics, 23(4):1249-1274.

McDonald, C. (2013). Migration and the housing market. Analytical Note AN2013/10, Reserve Bank of New Zealand.

Mendoza, E. G. (2006). Lessons from the debt-deflation theory of sudden stops. American Economic Review, 96(2):411-416.

Mendoza, E. G. (2008). Sudden stops, financial crises and leverage: A Fisherian deflation of Tobin's Q. NBER Working Papers 14444, National Bureau of Economic Research.

Merola, R. (2015). The role of financial frictions during the crisis: An estimated DSGE model. Economic Modelling, 48(C):70-82.

Minsky, H. P. (1975). John Maynard Keynes. The Macmillan Press Ltd, Basingstoke.

Mitchell, J., Pain, N., and Riley, R. (2011). The drivers of international migration to the UK: A panel-based Bayesian averaging approach. The Economic Journal, 121(557):1398-1444.

Modigliani, F. and Brumberg, R. H. (1954). Utility analysis and the consumption function: An interpretation of cross-section data. In Kurihara, K. K., editor, Post-Keynesian Economics, pages 388-436. Rutgers University Press, New Brunswick, New Jersey.

Monacelli, T. (2005). Monetary policy in a low pass-through environment. Journal of Money, Credit and Banking, 37(6):1047-1066.

Morris, S. D. (2016). VARMA representations of DSGE models. Economics Letters, 138:30-33. 
Müller, U. K. (2012). Measuring prior sensitivity and prior informativeness in large Bayesian models. Journal of Monetary Economics, 59(6):581-597.

Murchison, S. and Rennison, A. (2006). ToTEM: The Bank of Canada's new quarterly projection model. Technical Report 97, Bank of Canada.

Muth, J. F. (1960). Optimal properties of exponentially weighted forecasts. Journal of the American Statistical Association, 55:299-306.

Muth, J. F. (1961). Rational expectations and the theory of price movements. Econometrica, 29:315-335.

Mutschler, W. (2015a). Identification of DSGE models - The effect of higherorder approximation and pruning. Journal of Economic Dynamics and Control, 56:34-54.

Mutschler, W. (2015b). Local Identification of Nonlinear and Non-Gaussian $D S G E$ models. PhD thesis, Westfälische Wilhelms-Universität Münster.

Nason, J. M. and Smith, G. W. (2008). Identifying the New Keynesian Phillips curve. Journal of Applied Econometrics, 23(5):525-551.

Nathan, M. (2014). The wider economic impacts of high-skilled migrants: A survey of the literature. IZA Journal of Migration, 3(4):1-20.

Nelson, C. R. (1972). The prediction performance of the FRB-MIT-PENN model of the U.S. economy. American Economic Review, 62(5):902-17.

Nolan, C. and Thoenissen, C. (2009). Financial shocks and the US business cycle. Journal of Monetary Economics, 56(4):596-604.

Peri, G. (2016). Immigrants, productivity, and labor markets. The Journal of Economic Perspectives, 30(4):3-29.

Pesaran, M. H. (1987). The Limits to Rational Expectations. Basil Blackwell, Oxford.

Phelps, E. S. (1968). Money-wage dynamics and labor-market equilibrium. Journal of Political Economy, 76(4):678-711. 
Phillips, A. W. W. (1958). The relation between unemployment and the rate of change of money wage rates in the United Kingdom, 1861-1957. Economica, 25(100):283-299.

Phillips, P. C. and Ouliaris, S. (1990). Asymptotic properties of residual based tests for cointegration. Econometrica, 58(1):165-193.

Phillips, P. C. B. (1986). Understanding spurious regressions in econometrics. Journal of Econometrics, 33(3):311-340.

Plott, C. and Smith, V. (2008). Handbook of Experimental Economics Results, volume 1. North Holland, Amsterdam.

Poirier, D. (1998). Revising beliefs in nonidentified models. Econometric Theory, 14(4):483-509.

Poot, J. and Stillman, S. (2016). Skill composition of immigration flows and the measurement of education-occupation mismatch. IZA Journal of Migration, 5(18):1-23.

Preston, A. J. (1978). Concepts of structure and model identifiability for econometric systems. In Begstrom, A. R., Catt, A. J. L., Peston, M. H., and Silverstone, B. D. J., editors, Stability and inflation, pages 275-297. John Wiley and Sons, Chichester.

Qin, D. (1989). Fomalization of identification theory. Oxford Economic Papers, 41(1):73-93.

Qu, Z. and Tkachenko, D. (2012). Identification and frequency domain quasimaximum likelihood estimation of linearized dynamic stochastic general equilibrium models. Quantitative Economics, 3(1):95-132.

Radner, R. (1968). Competitive equilibrium under uncertainty. Econometrica, 36(1):31-58.

Ramey, V. (2016). Macroeconomic shocks and their propagation. In Taylor, J. and Uhlig, H., editors, Handbook of Macroeconomics, volume 2A, chapter 2, pages 71-162. Elsevier, Amsterdam. 
Ratto, M. (2008). Analysing DSGE models with global sensitivity analysis. Computational Economics, 31(2):115-139.

Ravenna, F. (2007). Vector autoregressions and reduced form representations of DSGE models. Journal of Monetary Economics, 54(7):2048-2064.

Robert, C. P. and Casella, G. (2004). Monte Carlo Statistical Methods. Springer, New York, 2 edition.

Romer, D. (1996). Advanced Macroeconomics. McGraw-Hill, New York.

Rothenberg, T. J. (1971). Identification in parametric models. Econometrica, $39(3): 577-591$.

Rubio-Ramírez, J. F., Waggoner, D. F., and Zha, T. (2010). Structural vector autoregressions: Theory of identification and algorithms for inference. The Review of Economic Studies, 77(2):665-696.

Rudin, W. (1976). Principles of Mathematical Analysis. McGraw-Hill, New York, 3rd edition.

Saiz, A. (2003). Room in the kitchen for the melting pot: Immigration and rental prices. Review of Economics and Statistics, 85(3):502-521.

Samuelson, P. A. (1961). Economics: An Introductory Analysis. McGrawHill, New York, 5th edition.

Samuelson, P. A. and Solow, R. M. (1960). Analytical aspects of anti-inflation policy. American Economic Review, 50(2):177-194.

Sargent, T. J. (1989). Two models of measurements and the investment accelerator. Journal of Political Economy, 97(2):251-287.

Sargent, T. J. and Wallace, N. (1975). "Rational" expectations, the optimal monetary instrument, and the optimal monetary supply rule. Journal of Political Economy, 83(2):241-254.

Schmitt-Grohé, S. and Uribe, M. (2003). Closing small open economy models. Journal of International Economics, 61(1):163-185. 
Schmitt-Grohé, S. and Uribe, M. (2004). Solving dynamic general equilibrium models using a second-order approximation to the policy function. Journal of Economic Dynamics and Control, 28(4):755-775.

Schmitt-Grohé, S. and Uribe, M. (2012a). Implementing Iskrev's identifiability test. Mimeo, Columbia University.

Schmitt-Grohé, S. and Uribe, M. (2012b). What's news in business cycles. Econometrica, 80(6):2733-2764.

Schorfheide, F. (2000). Loss function based evaluation of DSGE models. Journal of Applied Econometrics, 15(6):645-670.

Sims, C. A. (1980). Macroeconomics and reality. Econometrica, 48(1):1-48.

Sims, C. A. (2002). Solving linear rational expectations models. Computational Economics, 20:1-20.

Sleeman, A. G. (2011). The Phillips curve: A rushed job? The Journal of Economic Perspectives, 25(1):223-237.

Slobodyan, S. and Wouters, R. (2012). Learning in a medium-scale DSGE model with expectations based on small forecasting models. American Economic Journal: Macroeconomics, 4(2):65-101.

Smets, F. and Wouters, R. (2003). An estimated stochastic dynamic general equilibrium model of the euro area. Journal of the European Economic Association, 1:1123-75.

Smets, F. and Wouters, R. (2007). Shocks and frictions in US business cycles: A Bayesian DSGE approach. American Economic Review, 97(3):586-606.

Stähler, N. (2017). A model-based analysis of the macroeconomic impact of the refugee migration to Germany. Discussion Papers 05/2017, Deutsche Bundesbank.

Stigler, G. J. (1947). Professor Lester and the marginalists. American Economic Review, 37(1):154-157. 
Stiglitz, J. E. (2018). Where modern macroeconomics went wrong. Oxford Review of Economic Policy, 34(1-2):70-106.

Stillman, S. and Maré, D. C. (2008). Housing markets and migration: Evidence from New Zealand. Working Papers 08_06, Motu Economic and Public Policy Research.

Stock, J. H. and Watson, M. W. (1988). Testing for common trends. Journal of the American Statistical Association, 83(404):1097-1107.

Suh, H. and Walker, T. B. (2016). Taking financial frictions to the data. Journal of Economic Dynamics and Control, 64:39-65.

Taylor, J. B. (1979). Staggered wage setting in a macro model. American Economic Review, 69:108-113.

Tchatoka, F. D., Groshenny, N., Haque, Q., and Weder, M. (2017). Monetary policy and indeterminacy after the 2001 slump. Journal of Economic Dynamics and Control, 82:83-95.

Townsend, R. M. (1979). Optimal contracts and competitive markets with costly state verification. Journal of Economic Theory, 21(2):265-293.

Trefethen, L. N. and Bau, III, D. (1997). Numerical Linear Algebra. Society for Industrial and Applied Mathematics, Philadelphia, PA.

Vehbi, T. (2016). The macroeconomic impact of the age composition of migration. Analytical Note AN2016/03, Reserve Bank of New Zealand.

Wechsler, S., Izbicki, R., and Esteves, L. G. (2013). A Bayesian look at nonidentifiability: A simple example. The American Statistician, 67(2):90 93.

Weiske, S. (2017a). On the macroeconomic effects of immigration: A VAR analysis for the US. Mimeo.

Weiske, S. (2017b). Population growth, the natural rate of interest and inflation. Mimeo. 
Williamson, S. and Wright, R. (2010). New monetarist economics: Methods. Federal Reserve Bank of St. Louis Review, 92(4):265-302.

Williamson, S. and Wright, R. (2011). New monetarist economics: Models. In Friedman, B. M. and Woodford, M., editors, Handbook of Monetary Economics 3A, pages 25-96. Elsevier, San Diego, CA.

Woodford, M. (2003). Interest and Prices: Foundations of a Theory of Monetary Policy. Princeton University Press, Princeton, NJ.

Working, E. J. (1927). What do statistical "demand curves" show? Quarterly Journal of Economics, 41(2):212-235.

Wren-Lewis, S. (2018). Ending the micro-foundations hegemony. Oxford Review of Economic Policy, 34(1-2):55-69.

Wright, R. (2018). On the future of macroeconomics: A new monetarist perspective. Oxford Review of Economic Policy, 34(1-2):107-131. 